\title{
Capturing Chemistry in Action with Electrons: Realization of Atomically Resolved Reaction Dynamics
}

\author{
Anatoly A. Ischenko, ${ }^{\dagger}$ Peter M. Weber, ${ }^{\ddagger}$ and R. J. Dwayne Miller*, ${ }^{\prime}, \|_{\odot}$ \\ ${ }^{\dagger}$ Institute of Fine Chemical Technologies, Moscow Technological University, Vernadskogo 86, 119571 Moscow, Russia \\ ${ }^{\ddagger}$ Department of Chemistry, Brown University, 324 Brook Street, 02912 Providence, Rhode Island, United States \\ ${ }^{\S}$ The Max Planck Institute for the Structure and Dynamics of Matter, Hamburg Centre for Ultrafast Imaging, Luruper Chaussee 149, \\ 22761 Hamburg, Germany \\ "Departments of Chemistry and Physics, University of Toronto, 80 St. George, M5S 3H6 Toronto, Canada
}

\section{Supporting Information}

ABSTRACT: One of the grand challenges in chemistry has been to directly observe atomic motions during chemical processes. The depiction of the nuclear configurations in space-time to understand barrier crossing events has served as a unifying intellectual theme connecting the different disciplines of chemistry. This challenge has been cast as an imaging problem in which the technical issues reduce to achieving not only sufficient simultaneous space-time resolution but also brightness for sufficient image contrast to capture the atomic motions. This objective has been met with electrons as the imaging source. The review chronicles the first use of electron structural probes to study reactive intermediates, to the development of high bunch charge electron pulses with sufficient combined spatial-temporal resolution and intensity to literally light up atomic motions, as well as the means to characterize the electron pulses in terms of temporal brightness and image reconstruction. The use of femtosecond Rydberg spectroscopy as a novel means to use internal electron scattering within the molecular reference frame to obtain similar

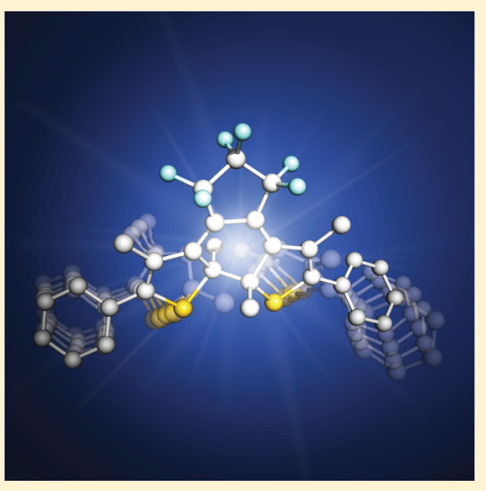
information on reaction dynamics is also discussed. The focus is on atomically resolved chemical reaction dynamics with pertinent references to work in other areas and forms of spectroscopy that provide additional information. Effectively, we can now directly observe the far-from-equilibrium atomic motions involved in barrier crossing and categorize chemistry in terms of a power spectrum of a few dominant reaction modes. It is this reduction in dimensionality that makes chemical reaction mechanisms transferrable to seemingly arbitrarily complex (large $\mathrm{N}$ ) systems, up to molecules as large as biological macromolecules $(N>1000$ atoms). We now have a new way to reformulate reaction mechanisms using an experimentally determined dynamic mode basis that in combination with recent theoretical advances has the potential to lead to a new conceptual basis for chemistry that forms a natural link between structure and dynamics.

\section{CONTENTS}

1. Introduction

Chemists' Gedanken Experiment: Challenge and Intellectual Objectives

Evolution in Electron Source Brightness: Realization of Atomically Resolved Reaction Dynamics

Toward a Reaction Mode Basis for Conceptualizing Chemistry

2. Theory Elements

2.1. Phototriggered Reaction Dynamics: Connection to Thermally Sampled Reaction Coordinates

2.2. Conical Intersections-Conceptualizing Reaction Coordinates

2.3. Recent Advances in Time-Dependent Ab Initio Methods

2.4. Theory of Pump-Probe Electron Diffraction: Inversion Problem
2.5. Cumulant Analysis of Gas Phase Electron Diffraction Data

Quasi-Rigid Molecules

Analysis of Intramolecular Motions

Construction of the Hamiltonian

Adiabatic Potential Energy Surface: Small

Amplitude Vibrations

Large Amplitude Motion

Nonlinear Molecules

Linear Molecules

2.6. Dynamics of Dissociation

2.7. Solution of the Inversion Problem for Gas Phase Systems

2.8. Difference Method for Time-Dependent Diffraction Data Analysis

Special Issue: Ultrafast Processes in Chemistry 
2.9. Time-Dependent Diffraction from Single Crystals: Solution to the Inversion Problem

3. Path to Atomically Resolved Reaction Dynamics 3.1. Structures of Transient Intermediate States

3.2. Molecular Alignment: Prospect for Improved Structural Determination for Gas Phase Systems

3.3. Scaling to Larger Systems: Electrocyclic Ring-Opening Reaction of 1,3-Cyclohexadiene

4. Breaking the Picosecond Barrier to Atomically Resolved Dynamics

4.1. Development of Ultrabright Femtosecond Electron Sources

High Brightness, Nonrelativistic Electrons Relativistic Electrons

4.2. Realization of Atomically Resolved Reaction Dynamics: Imaging Chemistry in Action

4.3. Alternative Electron Imaging of Reaction Dynamics: Rydberg Spectroscopy: Looking from the Inside Out

5. Summary and Future Outlook

Associated Content

Supporting Information

Author Information

Corresponding Author

ORCID

Notes

Acknowledgments

References

\section{INTRODUCTION}

\section{Chemists' Gedanken Experiment: Challenge and Intellectual Objectives}

One of the dream experiments in chemistry is to directly observe atomic motions in real time during chemical reactions. ${ }^{1,2}$ Chemistry after all involves structural dynamics by definition, and to observe the interconversion of molecules from one structure to another would capture the very essence of chemistry. It is well-appreciated that each subdiscipline in chemistry has unique intellectual targets. However, the entire field of chemistry is unified through a thought experiment in which we conceptualize how molecules interconvert between different structures during passage through the transition state region. This conceptualization of the very moment of chemistry is an important pedagogical tool as it directs attention to the relative arrangement of atoms defining the barrier to the reaction of interest. ${ }^{3}$ It is the control over the barrier height that allows one form of chemistry, out of the multitude of possible rearrangements of the atoms, to occur faster than competing processes. That is, chemistry by its very nature is a "race against time". The barrier height exponentially defines the statistical probability of a given pathway. It is the manipulation of barrier heights that gives chemists effectively exponential control over chemical outcomes. ${ }^{5}$

This classic thought experiment also touches upon one of the most significant intellectual questions in chemistry. For a system of $\mathrm{N}$ atoms, there are on the order of $3 \mathrm{~N}$ independent degrees of freedom, or dimensions, to chemical problems. The nuclear fluctuations leading to the chemistry involve highly nonlinear couplings of all these degrees of freedom. The current chemical synthesis problems of interest involve hundreds of atoms, with the current objective to obtain synthetic analogues of biological systems or nanometer scale active chemistry such as molecular motors. ${ }^{6}$ Even for relatively small molecular systems by today's standard, say 100 atoms, the number of possible nuclear configurations with local minima is nearly innumerable. It is clear then that the structural evolution of molecules along reaction coordinates involves only a subset of the $3 \mathrm{~N}$ modes, defined as the reaction modes. There must, therefore, be an enormous reduction in dimensionality at barrier crossing regions that counters the effects of scaling on the many body potential to nearly arbitrary levels of complexity (large $\mathrm{N}) .^{2}$ In the context of evolutionarily optimized biological systems that exploit chemical processes to drive functions, the specific physics involved must be robust even to the level of biological systems $(N>1000)$. How is this possible?

The answer to this question must involve barrier crossing processes whose relationship must be weighted in relation to competing processes. The reactive crossing is defined by farfrom-equilibrium, highly anharmonic motions, that are most strongly coupled to the reaction coordinate. It is the very anharmonicity in the many-body potential at the transition state that mixes the modes, leading to highly localized motions along reaction coordinates and the reduced dimensionality. ${ }^{2}$ The basic physics is shown in Figure 1 for the simplest case of electron transfer for both intermolecular and intramolecular processes. One has to consider both the electronic and nuclear degrees of freedom and their couplings in depicting reaction coordinates. The nuclear motions affect the relative energetics of the electronic states and thereby the potential energy surface for the system. In principle, all of the $3 N-6$ modes are coupled to the reaction coordinate. The number of degrees of freedom involved moreover needs to include bath modes if significant rearrangement of the surroundings stabilizes the product states (e.g., in electron transfer or changes in charge distribution). The number of degrees of freedom coupled to the reaction coordinate can become enormous, especially in condensed phase processes. However, there are certain motions that are more strongly coupled to the electronic structure of the reactive complex than others, as shown in Figure 1.

Figure 1A shows the important case for homogeneous electron transfer in solution. This is the simplest chemical reaction (no bonds are made or broken) in which only an electron is exchanged, in this case between two metal centers. The point of this figure is to show how the solvent fluctuations lead to instantaneous changes in the local charge distribution or electric field, which modulates the relative electronic levels. For a given spatial separation between the donor and acceptor, there is an electronic couping $\mathrm{V}_{12}$ defined by the exponentially decaying electron wave function overlap between the two sites. At the instant the solvent generates a charge distribution that brings the donor and acceptor states into resonance (point B), there is a probability for electron transfer determined by $\left|V_{12}\right|^{2}$, which defines the efficiency of passage. The time scale for relaxation from the critical point $(\mathrm{B})$ is determined by the solvent fluctuations along the coordinate that leads to reorganization of the medium stabilizing the electron on the acceptor site. The figure illustrates schematically how the nuclear motions along the reaction coordinate determine the dynamics and how these motions in turn are coupled to electronic states affecting the potential energy surface directing the reaction. Not all motions affect the cavity polarization. The key motions involve librations of the polar solvent molecules 
(meant to emulate water), which most strongly affect the local field stabilizing the charge separation. For liquids, the specific details beyond this level of description are lost in the broad distribution of possible solvent configurations. The situation can be much better defined in going to solid state systems or intramolecular reactions. Figure $1 \mathrm{~B}$ shows the case for intramolecular charger transfer as another limiting case for formally the same class of reaction (i.e., electron transfer in which intramolecular reorganization of nuclear configurations dominate the stabilization of the spatial change in electron distribution). Figure $1 \mathrm{~B}$ shows the strong modulaton of the electronic surface that occurs for only a few key modes, even in the limit of a statistical distribution of vibrational energy. ${ }^{8}$ There are only a few, key frequencies within this distribution that most strongly couple to the reaction coordinate.

As another example of an important problem, with increasing levels of complexity (large $\mathrm{N}$ ), very similar behavior is found for the excitation of retinal within rhodopsin or bacteriorhodopsin ${ }^{9}$ as part of the primary step of vision and energy transduction in biological sytems, respectively. The partitioning of energy very rapidly evolves to a statistical distribution as typically assumed in transition state theory. Even in this statistical limit, one can see a strong modulation of the barrier separating two stable ground state structures at a particular $\mathrm{C}=\mathrm{C}$ stretch frequency representing bond elongation or bond softening and a very strong lower frequency torsional modulation that is necessarily involved in the isomerization process. ${ }^{9}$ These two modes define the coupling mode and the tuning reaction mode (twisting in this case) in this classic reaction. At the barrier crossing region, the very strong anharmonicity in the potential energy surface couples a high frequency and low frequency mode, leading to the relatively localized motions giving rise to the chemistry. This reduction to a few key modes occurs for systems as large and complex as proteins.

The main intellectual challenge is to understand the generalized principles that reduce the controlling of chemistry to a few key modes and to apply them to predict different classes of reactions. We know from work back in the 1970's involving the study of vibrational overtones that anharmonicity in even a single coordinate leads to the formation of local modes (i.e., molecular motions that are highly localized at specific regions of a molecule). ${ }^{10}$ The system rapidly relaxes from such points, and it is the coupling to additional vibrational modes that leads to rapid intramolecular vibrational relaxation (IVR). ${ }^{11,12}$ No chemical reaction, other than in diatomics, occurs through the displacement of a single vibrational mode. Reactions must involve more than one degree of freedom, just as in classical mechanical systems where material fracture or cutting with scissors ${ }^{13}$ must involve shear motions as well as displacive motions. It is in fact the very anharmonic motions at far-from-equilibrium points in the nuclear configurations that dramatically reduce the $3 \mathrm{~N}$ dimensional problem to the few localized motions involved in the reaction.

Figure 2 illustrates how the near-equilibrium motion of an effective mass executing fluctuations within linear, or harmonic limits, gives rise to a single frequency involving order $3 \mathrm{~N}$ motions for an $N$-body system. The far-from-equilibrium motion of this same effective mass takes on additional Fourier components near the barrier region. One can readily appreciate that the anharmonicity of the potential energy surface mixes high frequency with low frequency modes, which may lead to highly localized motions. The crossing to a new stable minimum of the product channel is seen to be analogous to the local modes observed in vibrational overtone spectroscopy. These motions are statically weighted with respect to the barrier height, in large part defined by these motions, to give an exponential dependence of the chemical dynamics in competition with other channels.

The basic idea of anharmonic coupling leading to a reduced coordinate space comprising the reaction modes seems simple enough. The main problem is that based on static structures alone, one cannot infer what the active coordinate space is. This reflects the high dimensionality of the problem: there are so many ways to combine different nuclear motions to find the same minima that it is impossible to know a priori which are involved in the reactive pathways. Theory encompassing a full modal analysis is only starting to be sufficiently accurate to get qualitative pictures of the key modes. As a consequence, there have been vigorous debates about the key motions involved in the molecular dynamics within the barrier-crossing region. For typical systems, one has to use highly simplified models of what might be the reactive space. The concept of bicycle pedal motion over pure rotatory motion in the classic, still ongoing, debate of the primary step in vision is just one example. ${ }^{14}$

If we could image chemical processes at the atomic level of detail, we would be able to observe the reactive modes in action and directly determine their coordinate space. Moreover, one could extract directly the reaction forces, yielding the effective mass and the time-dependent velocities of these motions. Such a measurement, by definition, gives the gradients in the potential surface that drive the atomic displacements. In chemical physics, it is traditional to represent reaction coordinates as potential energy surfaces. If we can directly observe the time-dependent atomic motions, it would be possible to construct relevant potential energy surfaces for even arbitrarily complex molecular systems in a single measurement. This statement requires solution to the inversion problem in time-resolved diffraction, which as will be discussed below, is possible in time-resolved measurements as one is not trying to solve a completely unknown structure. By taking advantage of either the known initial structure and conserved structural components or use of theoretically calculated potential energy surfaces for refinement, it is now possible to obtain atom resolved chemical reactions within robust constraints.

Directly observing the reaction forces in this manner gives us the very essence of chemistry. Moreover, the direct observation of the key modes or coordinate space controlling the reaction enables a determination of the critical nuclear configuration defining the barrier, which in turn provides the insights needed to better control the barrier height and with it the chemical process of interest. For example, one might place polar residues near regions requiring the stabilization of charge separation or bond softening involving polar modes to lower the barrier and more efficiently obtain the desired product. For this reason, the conceptualization of the structure of the barrier crossing, or transition state region, is taught at the earliest levels of chemistry. It enables one to think about new means to control chemical reactions. With atomically resolved reaction dynamics, we are now moving beyond conjecture to a complete picture of the motions involved. In order to arrive at this point in our understanding and ability to control chemistry, it is essential to directly observe the highly correlated relative atomic motions that make up these reaction modes, as will be highlighted below.

Let us put the power of controlling barrier heights into context. For example, there are certain quantum effects that 
control material properties. Electron-electron correlations and spin correlations, through various mechanisms, lead to changes in the colossal magnetoresistance for materials on the order of $10^{8}$ to $10^{10} .15$ The exponential gain in laser amplifiers due to cascaded stimulated photon emission leads to overall gains of more than $10^{6}{ }^{16}$ We find wonder in these enormous gains through some coherence effect but do not realize or take for granted that very similar gains are routinely manipulated through chemistry to control molecular properties. Each molecule has a different shape and properties precisely due to electron correlations that lead to more stable spatial configurations of electron and nuclear probability distributions. Differences in reaction rates can be made to vary by $10^{22}$ or more for typical catalysts that lower barriers to specific chemical processes. ${ }^{17}$ Each step in an overall synthetic route to specific structure further amplifies the contrast in the desired pathway over competing pathways. Chemists literally have a control knob for directing chemistry with exponential gain that covers many orders of magnitude. This is a truly remarkable degree of control over the structure of matter. It is well-appreciated that this degree of control similarly reflects electron correlation effects that favor one arrangement of nuclei over another. Given this, each molecular system represents a unique many-body system, and one might expect each synthetic route to require a unique solution. This point is made in light of the high dimensionality of the problem, as discussed above, and the role of anharmonicity and electron correlation effects in defining the control problem of the transition state region. Each molecule has a specific shape and electron correlation energies. Even enantiomers with the same composition can have different shapes and significantly different chemical properties. For example, lemon and orange esters are enantiomers that smell dramatically different because of variations in their binding to olafactory sensors. The shape and attendant differences in electron distribution gives rise to a multitude of different chemical properties and functions.

Nevertheless, despite the complex nonlinear nature of chemistry, a large library of synthetic strategies has been developed, suggesting that a large degree of generalization exists. ${ }^{18}$ These reaction mechanisms have been discovered either empirically, or one could argue fortuitously, with oversimplified assumptions for the nuclear configurations of the transition state region with effectively no information on the sampling of this nuclear configuration. Major questions remain about what makes chemistry a transferable concept, especially in light of the highly nonlinear nature of complex many-body interactions and the relative robustness with respect to scaling to more complex systems. The answer to these questions will enable chemistry to make the leap in de nouvo synthetic strategies to complex systems comparable to biological systems. We are currently missing key information needed to make this transition. The importance of directly observing chemical reaction dynamics at the atomic level needs to be viewed in this context.

The prospect of obtaining sufficient space-time resolution to literally watch atomic motions was considered to be impossible, relegating the atomic level details of reaction dynamics to be inferred from a culmination of indirect methods. This point was effectively made with respect to the importance of perturbative methods to probe reaction dynamics. ${ }^{19}$ Indeed, great progress has been made in combining spectroscopy and various versions of molecular beam methods to get sufficient information to construct reaction diagrams in combination with theory. ${ }^{20}$ This approach is best suited for small molecular systems and does not address the seeming robustness of chemistry in scaling to evermore complex systems. Also, these measurements can identify a certain subset of reaction modes (those that are amenable to spectroscopic probes) but not the displacements of atoms along all coordinates, which is the essential information. If one could directly observe reactions at the atomic level, on the primary time scales of barrier crossing events, we would have all the information needed to optimize the chemical mechanisms. We now have this capability.

\section{Evolution in Electron Source Brightness: Realization of} Atomically Resolved Reaction Dynamics

The key enabling technology to address this challenge to experimental physical chemistry was the development of electron sources sufficiently bright to literally light up atomic motions. The path toward this achievement commenced with comparatively low brightness sources that followed in parallel to the development of light sources for spectroscopy. Ischenko et al. performed the first experiments that laid the foundation for the methodology and technology to follow. ${ }^{21-24}$ They used a pump-probe method with a laser pulse to induce the dynamics and an electron pulse as a structural probe. A deflection method enabled them to achieve microsecond time resolution. This was sufficient to determine the structures of transient intermediates in the photochemistry of halomethane systems and different fragmentation pathways involved in the photodissociation of $\mathrm{CS}_{2}$. This work illustrated the promise of structural probes to study photoreactive intermediates. The next important development was the use of pulsed electron sources to study gas phase photochemistry by Williamson et al., ${ }^{25}$ Ischenko et al., ${ }^{22-24}$ and Dudek et al. ${ }^{26}$ The Zewail group was the first to cross the threshold to few picosecond time resolution. $^{25}$ Given the low signal-to-noise for gas phase electron diffraction experiments, these accomplishments ${ }^{21-26}$ are all the more remarkable in terms of detecting the small differential changes in diffraction intensity to connect to structural intermediates. This pioneering work still sets the standard for studies of gas phase photochemistry. The timeresolution, limited by both the electron pulse durations and the velocity mismatch between the laser excitation and the electron probe, $^{27}$ was sufficient to capture transient intermediate structures but was not sufficient for observing the correlated atomic motions leading to the reaction intermediates. The first experiments to break the picosecond barrier with sufficient electron brightness were those of Siwick et al., ${ }^{28}$ who were able to follow the relative atomic motions involved in melting under strongly driven conditions. Bond breaking and shear motions were resolved faster than collisional processes would blur the details of atomic motions. The time resolution reached $600 \mathrm{fs}$, even with an electron source intense enough to enable single shot measurements (the detector had only $10 \%$ quantum efficiency, thus requiring more shots). The most important feature of this work was the realization that an electron source with brightness sufficient for single shot measurements is essential to the study of irreversible chemical processes. Subsequent increases in electron source brightness and improvements of the time-resolution culminated in the first atomically resolved reactions and the direct observation of the key modes involved in the reactions, as will be elaborated upon below.

An important consideration for measuring structural dynamics is an experiment's time resolution. However, there 


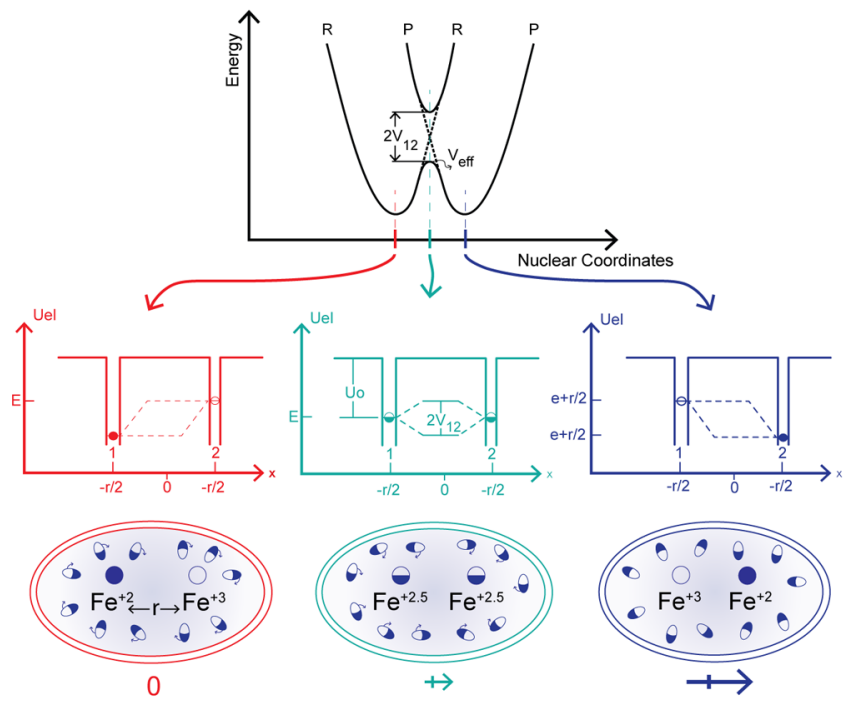

b
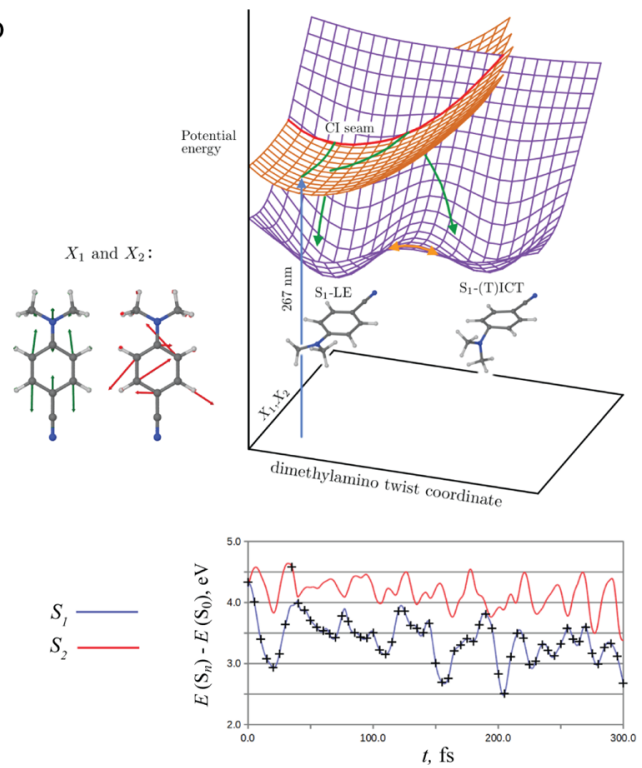

Figure 1. Electron Transfer Example. (a) Diagram representing the potential energy surfaces for $\mathrm{Fe}^{2+} / \mathrm{Fe}^{3+}$ metal-to-metal electron transfer. The problem is symmetric with only solvent fluctuations leading from reactant state to the barrier where the solvent fluctuations bring the donor and acceptor levels into resonance as needed for electron transfer. If the electron coupling $\left|V_{12}\right|$ is sufficiently strong, the electron transfer process adiabatically follows the fluctuations in barrier crossing to the product state. The microscopic motions most strongly coupled to the reaction coordinate are depicted as primarly the hindered rotations of an idealistic polar dipole for the solvent. Adapted with permission from ref 7 . Copyright 1995 John Wiley \& Sons. (b) A schematic illustration of the relaxation dynamics of dimethylaminobenzonitrile (DMABN) in the gas phase following photoexcitation to the $S_{2}$ state. $X_{1}$ and $X_{2}$ are the branching space coordinates, which lift the degeneracy at the $S_{2} / S_{1}$ conical intersection $(\mathrm{CI})$ seam. The dimethylamino group twisting coordinate, which is orthogonal to $X_{1}$ and $X_{2}$, is responsible for the interconversion between the $S_{1}$-LE and $S_{1}$ (T) intramolecular charge transfer structures (ICT). The relevant motions are indicated. The inset shows the energies of the $S_{2}$ and $S_{1}$ states relative to the ground state during a simulated relaxation trajectory in which one clearly sees distinct modulation arising from only a few modes within the distribution that ultimately direct the chemistry. Adapted from ref 8. Copyright 2015 American Chemical Society.
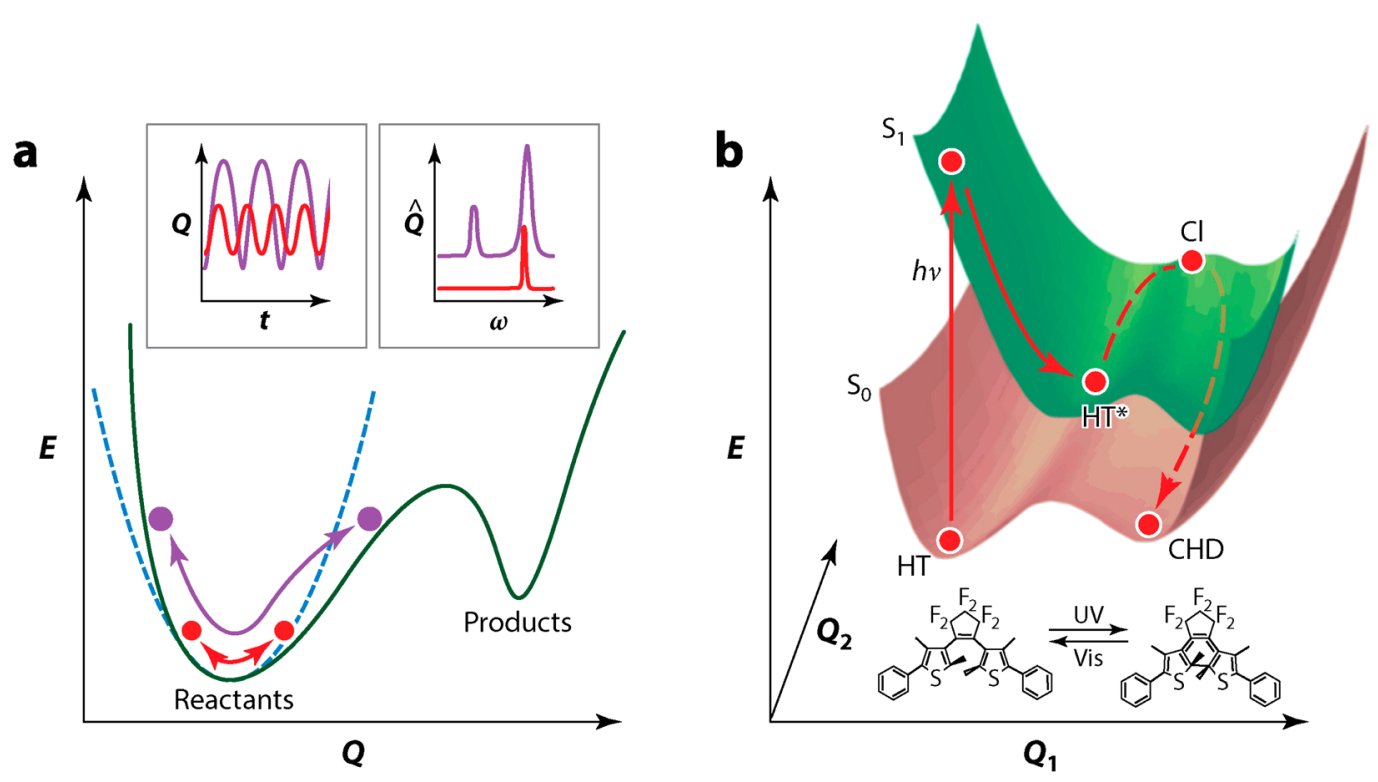

Figure 2. (a) The motion of an effective mass along one slice of a many-body potential representing the motion along a particular mode. The relatively small displacements (red) are well-defined by harmonic motions. However, far from equilibrium, the motion of the effective mass becomes more complex (purple). The motion shows multiple frequency components as the object executes its motion on the surface and samples the highly anharmonic components of the potential energy surface. The motions in time, and the decomposition into frequency components, are shown in the upper insets. (b) The reduced potential energy surface for the ring closing reaction of diarylethene, as shown by the molecular structures, illustrates the preparation of the excited state in which the ensuing motions projected along two reduced reaction coordinates (Q1, Q2) define the dominant reaction modes. As per (a), the superposition of vibrational modes in this process leads to the localized motions of the chemical reaction. An enormous reduction in dimensionality can be rationalized in this way, but there is still ambiguity as shown by the arrows depicting possible pathways. The challenge is to identifying the key reaction modes and dynamical pathyways that enable a full understanding of the reaction mechanisms. Reproduced with permission from ref 2. Copyright 2014 Annual Reviews. 
has been a great deal of confusion in the literature in which the emphasis has been placed solely on electron pulse durations and the use of fully reversible processes to demonstrate the time resolution. Doubtless, there have been important proof of principle experiments using low brightness electron and X-ray structural probes to follow phonon motions. ${ }^{29,30}$ Little new information is, however, extracted from these studies. The observations are related to small atomic displacements in the fully reversible regime, in which the primary observable is the phonon frequency. This information could be more readily obtained by all-optical methods. The discussion of time resolution solely in terms of pulse durations then distracts from the real issues in casting the measurement as a problem in image reconstruction, with all the attendant problems in achieving sufficient brightness for contrast, and Fourier components to faithfully reproduce the image. It is ultimately electron source brightness that is the essential consideration and not just the pulse duration. The major milestones in the development of time-resolved electron diffraction for the study of chemical reaction dynamics are given in Figure 3, where the importance of source brightness is clearly demonstrated by considering only work in which structural changes were resolved.

To fully appreciate the importance of source brightness in imaging and the achievement of atomically resolved dynamics, consider the elements needed for a camera to make a "Molecular Movie". First consider the image source (lighting)

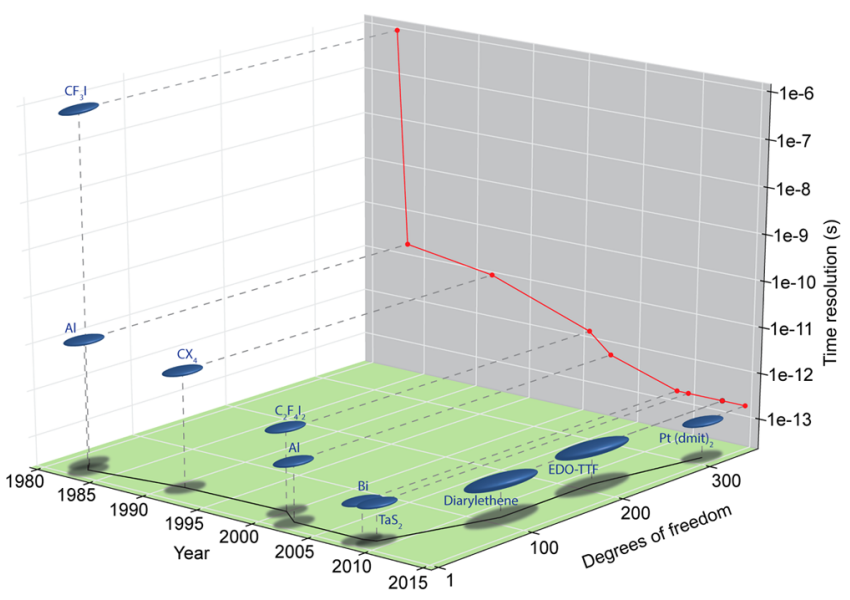

Figure 3. Major milestones in resolving structural changes with electrons. The importance of source brightness is evident as the picosecond barrier was broken by sources bright enough to enable the study of irreversible systems. This figure of merit is distinct from the study of reversible or thermal processes. $\mathrm{CF}_{3} \mathrm{I}$, with sub-ms time resolution, was reported in 1983 by Ischenko et al. ${ }^{21}$ The dynamics of heating $\mathrm{Al}$ near the onset of melting was reported by Williamson et al. ${ }^{31}$ in 1984 with a resolution of $\sim 10$ ps. A time-resolution of $<10$ ps was achieved in 1992 by Williamson et al. ${ }^{25}$ A 1 ps time resolution was achieved in 2001 by Ihee et al. ${ }^{32}$ The picosecond barrier was broken in 2003 by Siwick et al. ${ }^{28}$ Later on, in $2009, \mathrm{Bi}^{33}$ by Sciaini et al. to illustrate the fastest structural transition and $\mathrm{TaS}_{2}{ }^{34}$ by Eichberger et al. in 2010 improved the time resolution and diffraction quality with a more compact electron gun design. The first systems involving bond formation in electrocylization (diarylethene), ${ }^{35}$ intermolecular electron transfer (EDO-TTF) ${ }^{36}$ for organics, and metal-to-metal electron transfer $\left[\mathrm{Pt}(\mathrm{dmit})_{2}\right]^{37}$ exploited rf pulse compression ${ }^{35,36}$ or further compaction in electron source ${ }^{37}$ to increase source brightness. The observed trend gives a clear connection between source brightness and the ability to study larger systems. requirement for the spatial resolution to map chemical reaction pathways. To resolve the vibrational nonadiabtic coupling to reaction coordinates and the effect of vibrational anharmonicity requires sub- $\AA$ spatial resolution, in many cases better than 0.1 $\AA$. (For example, the net motion of the central carbon atoms involved in the photoisomerization of retinal is $0.1 \AA.)^{14}$ The central carrier wavelength of a pulsed source must therefore be on this length scale or shorter. Using diffraction methods, this spatial resolution requirement can be met with either hard Xray or high-energy electrons.

Now consider the time-resolution requirements. The relevant shutter speed can be estimated by considering the time it takes two atoms or reduced masses to move along a dissociative coordinate to a point where the binding energy is no longer within $\mathrm{kT} .{ }^{38}$ At this point in the reaction coordinate, one can say the bond is broken. For typical speeds $\left(\approx 10^{5} \mathrm{~cm} / \mathrm{s}\right)$ of sound defining thermal fluctuations, and potential energy surfaces for which motions on the order of $1 \AA$ are required to reach unbound parts of the potential, the relevant time scale is $100 \mathrm{fs}^{38}$ This time scale should be familiar. It is the typical sampling frequency of the Arrenhius expression for first-order rate expressions. While oversimplified, this estimate provides the relevant time scales. There are faster dissociative channels involving excited state potentials, on the order of $25 \mathrm{fs},{ }^{39,40}$ but most chemical reactions involve more long-range correlated motions of collective modes, especially in condensed phases where the most highly damped modes along the reaction coordinate occur in the 100 to $10 \mathrm{~cm}^{-1}$ range ( $\left.>100 \mathrm{fs}\right){ }^{2,41,42}$ The fastest chemical process known, that does not involve dissociation, is the primary step of vision involving photoinduced isomerization around the $\mathrm{C}_{11}-\mathrm{C}_{12}$ double bond of retinal. This time scale was initially assigned to occur on the 200 fs time scale, and it was one of the fastest chemical reactions known. The primary step of vision has now been shown to occur effectively coherently (ballistically) through the displacement of just 3 key modes on the time scale of $30 \mathrm{fs}^{43}$ This is one of the most highly optimized chemical processes known, especially given the complexity and scale of a biological system. All other chemical processes are typically slower, so that the 100 fs time scale serves as a useful metric for sufficient temporal resolution. To be clear on the required time resolution, it should be noted that there are faster nuclear motions. The fastest nuclear motion is the $\mathrm{OH}$ stretch of liquid water with a half period of $10 \mathrm{fs}$; however, the rms displacement is less than $0.05 \AA$, which is not a chemically relevant length scale relative to those associated with barrier crossing processes. It is the nuclear fluctuation time scale, during which the coupling of various vibrational modes leads to the highly anharmonic localized motions through the barrier region, that we call chemistry. These processes occur predominantly on the 100 fs time scale.

As a final point on the required time resolution, arguments have been presented that the attosecond time scale characteristic of electron dynamics is the primary process to be controlled and therefore relevant time scale. ${ }^{44}$ Attosecond methods provide important information on electron dynamics under high field conditions with numerous applications exploiting concepts of electron recollision physics. However, in chemical processes, the electronic degrees of freedom adiabiatically follow the nuclear motions, not the other way around. The heat capacity or thermal reservoir responsible for the nuclear motions giving rise to the chemistry is largerly determined by the system's internal energy within the nuclear 
degrees of freedom. This fact is part of the success of the Born-Oppenheimer approximation in treating molecular wave functions and dynamics. The only time the electron dynamics become slower than the nuclear time scale is at conical intersections (to be discussed below) or other related crossings where the mixing between electronic surfaces is small $(<100$ $\left.\mathrm{cm}^{-1}\right) .{ }^{45}$ However, even here it is the nuclear motions that bring the system to this point and define the operating time scale. The electron dynamics at this point in nuclear configurations determine the quantum efficiency at the singularity, not the effective time scale of the crossing. For the purposes of the present discussion, we use the $100 \mathrm{fs}$ time scale as the canonical time resolution needed to atomically resolve chemical reaction dynamics.

No detector is fast enough to resolve structural molecular dynamics on this time scale. One has to use perturbative methods to trigger the structural changes and then use a structural probe in a repetitive stroboscopic pump-probe protocol that stitches together a movie of the atomic motions. This approach is now standard in femtosecond spectroscopy. The time resolution is provided by sampling a system response by exciting the sample with an excitation pulse shorter than the dynamics of interest and then nonpertubatively sampling the ensuing dynamics with a variably time delayed, sufficiently short, probe pulse sensitive to the excited state processes. The associated time base is scanned using various strategies for data collection, averging, and maximing the signal-to-noise ratio of the time-dependent signal. A slow detector is used to read out the probe intensity at each time delay. The detector read-out only needs to be faster than the time taken to change the time delay between the excitation and probe sequence. The main problem in the past has been to develop short enough pulses to temporally resolve the dynamics of interest. In this light, one could argue that the main challenge is to obtain short enough pulses of either X-rays or electrons to capture the relevant motions. The $100 \mathrm{fs}$ time scale may seem incredibly fast for Xrays or electron imaging sources. However, there are wellestablished methods using either laser plasma or femtosecond photoemission methods, which mimic laser pulse durations to generate such sufficiently short pulses of X-rays or electrons, respectively. The real problem is the brightness. It took 20 years after the first femtosecond lasers to achieve sufficient source brightness. This issue can be most readily understood within this movie camera analogy. As one goes to shorter and shorter shutter speeds (higher and higher time resolution), one needs a brighter and brighter source to maintain image quality. The ability to observe edges and spatial relations depends on the contrast, which is related to the dynamic range of the detected image, which in turn depends on the brightness, or integrated detected flux, of the image. This problem was the driving force for the development of faster shutter speeds and flash bulbs in the early days of photography to freeze out motions. Brightness determines the signal-to-noise and contrast gradients in an image.

To better define the source brightness issue, we should consider the imaging method. Atomically resolved structures can be obtained using either diffraction (reciprocal space) or real space imaging methods. The signal for diffraction scales as $\mathrm{N}^{\prime 2}$, while real space imaging scales as $\mathrm{N}^{\prime}$, where $\mathrm{N}^{\prime}$ refers to the number of unit cells in a single crystal sample volume. ${ }^{46}$ This dependence is due to the fact that the diffracted signal field adds in quadrature to the contributing scattering terms to give the measured intensities of the diffraction orders. For this reason, diffraction experimentally scales quadratically with the sample thickness, or $\mathrm{N}^{\prime 2}$. This amplification factor makes diffraction orders of magnitude more sensitive than real space imaging, and within current source limitations is the only option for imaging atomic motions in real time. For simple unit cells (order 10 atoms/unit cell), the number of electrons required to achieve enough diffraction orders with sufficient dynamic range to resolve the relevant motions is on the order of $10^{4}$ detected electrons and scales approximately linearly with the number of atoms/unit cell. As an approximate rule of thumb, one needs $10^{5}$ electrons for typical molecular systems of interest with $\sim 100$ atoms/unit cell and $10^{7}$ electrons for systems comparable to proteins. This scaling can be gleaned from typical reported static diffraction patterns.

The real problem with the source brightness arises from the need to phototrigger the structural dynamics of interest. Chemical processes, or other structural transitions, are rare events and need to be phototriggered to provide a time origin. To get above background, the excitation level needs to be $\sim 10 \%$ for the sampled volume. Since chemical reaction dynamics are generally nonreversible, this level of excitation often leads to sample damage in a single laser shot. The significance of this point needs to be fully appreciated. The preparation of samples with uniform quality and thickness on the $10-100 \mathrm{~nm}$ thickness scale suitable for electron probes (transmission studies) is extremely demanding, and one quickly runs out of "film" before enough time points can be recorded to observe the relative atomic motions. Even in the limit of fully reversible systems, samples on this thickness scale take 10-100 $\mathrm{ms}$ time scales to recover and signal sampling is reduced to the $10 \mathrm{~Hz}$ range or slower, such that comparable signal-to-noise ratios (SNR) in imaging atomic motions takes quadratically longer to collect sufficient signal. From a practical standpoint, samples under irradiation have a finite lifetime such that the brighter the source the better the resolution and contrast to track atomic motions. Further, the 100 femtoseond timeresolution requirements (100 fs excitation pulse widths for triggering) and pulse energy needed to give this fraction of excited states leads to maximum peak powers on the order of $10-100 \mathrm{GW} / \mathrm{cm}^{2}$ for samples of a few micron thickness. Peak powers in this range lead to sample damage even in the case of otherwise reversible systems, which reinforces the need for source brightness capable of generating single pulses of either electrons or X-rays with sufficient intensity for single-shot structure determination.

All the technology was available in the 1980's to 1990's to do this class of experiment with low flux sources. ${ }^{21,30,31}$ But it is only recently that the brightness issue has been fully appreciated and solved. ${ }^{28,47}$ To emphasize this most critical technical point, it is not sufficient to have simultaneously sufficient spatial and temporal time resolution (femtosecond excitation, femtosecond structural probe pulses) but the structural probe must have sufficient brightness for single shot structure determination to follow chemical reactions. There are a few cases where photoreversible processes relax this condition (vide infra), but in all cases, thermal effects dictate the sampling rate, which makes the requirement of high brightness sources a general condition.

The major limitation in source brightness with electrons is the inherent Coulombic repulsion between electrons, or space charge effects, that limits the charge density per pulse for a given energy spread or brightness. It is fair to say that this limitation was thought to make it impossible for high bunch 
charge electron sources to achieve atomically resolved structural dynamics and provided great incentive for applications of X-ray free electron lasers (XFELs) in this domain. A major advance in electron source brightness came from an effectively exact solution to the coupled equations of motion for some $10^{4}$ electrons to simulate the electron pulse propagation dynamics with sufficient electron numbers for single shot structures. ${ }^{47}$ This work discovered that there are indeed very strong space charge temporal broadening effects but no significant loss of spatial coherence or resolving power at useable electron densities by using the shortest possible propagation distances (vide infra). The unexpected finding was that there is also no significant loss in space-time correlation for these conditions. The electrons at the front of a pulse are accelerated to higher velocity relative to the trailing electrons that are in like fashion decelerated to lower velocities. For nonrelativistic electrons, the higher-energy electrons have larger velocity than lower-energy electrons, which leads to a self-sorting of the electron energies such that the electron pulse develops a nearly perfectly linear chirp. The importance of this detail is shown in Figure 4 and is immediately apparent. This feature was missed in prior analytical treatments and could only be gleaned from accurate numerical simulations of the pulse propagation. It is immediately apparent from this work that such highly linearly chirped pulses can be readily recompressed at the sample position using a number of possible dispersive electron optics. The alternative and most robust solution also revealed from this work was to use extremely compact electron gun designs to minimize pulse broadening by limiting the propagation length. ${ }^{28,48-53}$ This study was also crucial in providing the essential information with respect to the experimental parameter space for the compact gun concept with sufficient electron numbers and coherence for single shot structure determination without the need for compression. This difference greatly simplies the gun design.

The effective source brightness made possible by this work needs to be fully appreciated. Single shot structure determination is now possible for even complex systems, as will be seen below (section 4.2). It is now routine to generate electron pulses with up to $10^{5}-10^{6}$ electrons per pulse, with $100 \mathrm{fs}$ pulse durations, with beam sizes on the order of $100 \mu \mathrm{m}$ that are ideally suited to match typical excitation profiles used for femtosecond spectrosopy. ${ }^{50}$ These parameters correspond to a peak current density of $1.6-16 \mathrm{kA} / \mathrm{cm}^{2}$, which is more than 4 orders of magnitude higher peak current density on target than typical TEM parameters used for imaging, with similar gains over previously used sources for time-resolved studies (compare Figure 1 in ref 48 to electron bunch charge densities of earlier work).

To fully quantify source brightness in terms of imaging atomic motions, it should be stressed that the ultimate limit in spatial resolution is determined by the transverse energy spread of the electron photoemission at the source. The transverse normalized rms emittance describing this distribution is defined by $^{54}$

$$
\varepsilon_{x} \equiv \frac{1}{m c} \sqrt{\left\langle x^{2}\right\rangle\left\langle p_{x}^{2}\right\rangle-\left\langle x p_{x}\right\rangle^{2}}
$$

where $m$ is the electron mass, $\mathrm{p}_{x}$ is the transverse moment, and $x$ is the position in the transverse direction, using $z$ for the propagation axis. The emittance in $y$ and $z$ are defined similarly, which delineates a 3D phase space for the electron bunch. The spatial resolution is determined by the transverse emittance.
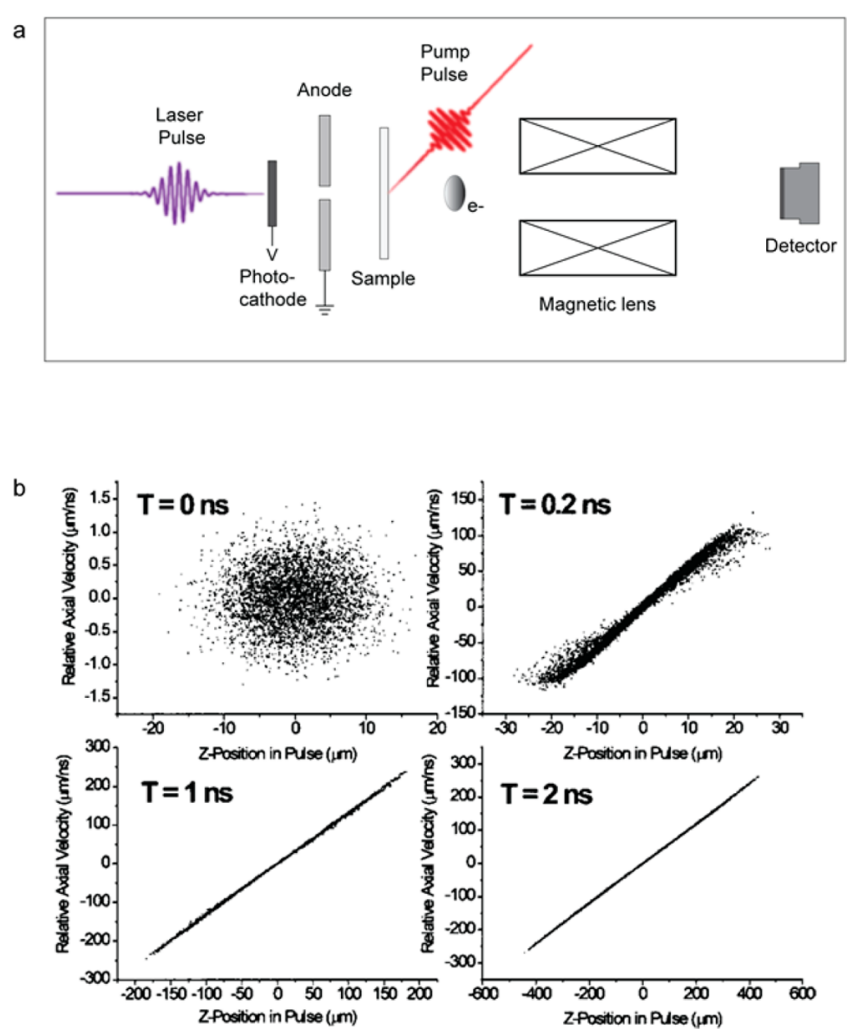

Figure 4. (A) The basic elements in a femtosecond electron gun. The photoinjection pulse impinges on the photocathode at high potential relative to an extraction grid at ground in which a collimating lens and aperture can be either before or after the sample as evident from this figure. (B) The use of the tree code with the above structure was used to calculate the electron propagation dynamics for the coupled equations of motions for $10^{4}$ electrons. This single result led to two new high intensity femtosecond electron source concepts. One can see that if the propagation distance from the photocathode is kept short, comparable to the extraction distance, pulses of sufficient bunch charge for single shot structure determination with $100 \mathrm{fs}$ time resolution can be generated. The onset of perfectly linear chirp is equally apparent and led to the idea of using dispersive pulse compression schemes to temporally refocus the electron pulse to give the highest possible source brightness at the sample position for given beam parameters. Reproduced with permission from ref 47 . Copyright 2002 AIP Publishing LLC.

For a Guassian distribution with an rms beam waist, $\sigma$, for the distribution in $x$, this relation reduces to $\varepsilon_{x}=(1 / \mathrm{mc}) \sigma_{x} \sigma_{\mathrm{px}}$ in which the transverse momentum spread $\left(\sigma_{\mathrm{px}}\right)$ can be defined by an effective electron temperature [i.e., $\sigma_{\mathrm{px}}=(\mathrm{mkT})^{1 / 2}$ ]. The transverse emittance at the source is then $\varepsilon_{\mathrm{x}, \mathrm{y}}^{\mathrm{s}}=(k T /$ $\left.\mathrm{mc}^{2}\right)^{1 / 2} \sigma_{\text {source }}$, with the source size for photoinjection given by the laser beam spot on the photocathode $\left(\sigma_{\text {source }}\right)$. One wants to have as small a transverse energy spread or lowest emittance possible for a given photoinjection spot size. The transverse moment component determines the beam divergence; the lower the emittance the more parallel the outgoing beam and consequently higher spatial coherence. It is the transverse component that determines the spatial resolution and the longitudinal distribution of electrons that determines the temporal resolution. The objective is to conserve the transverse emittance, keep it as low as possible at the sample position with the highest number density of electrons and in the process also minimize pulse broadening. The brightness of the electron 
source is usually characterized in terms of the reduced brightness using the above terms as

$$
B_{r} \equiv \frac{1}{U} \frac{\partial^{2} I}{\partial A \partial \Omega} \equiv \frac{\hat{I} m c^{2}}{2 \pi^{2} \varepsilon_{x} \varepsilon_{y}}
$$

where $U$ is the electron energy, $A$ is the beam area, $\Omega$ is the solid angle, $I$ the current, and $\hat{I}$ the peak current. The key parameter in relating emittance to spatial resolution is the transverse coherence length $\left[L_{\mathrm{T}} \equiv(h / 2 \pi) \sigma_{\mathrm{px}}\right]$, which from the above can be written as

$$
L_{\mathrm{T}}=\left[h / 2 \pi(k T)^{1 / 2}\right]\left(\sigma_{\text {sample }} / \sigma_{\text {source }}\right)
$$

There are issues of global versus local transverse coherence, but this simple expression gives an excellent approximation. In order to resolve structural changes, the transverse coherence of the probe electrons at the sample position must be on the order of the unit cell dimension to coherently scatter and report on the lattice. The reduced brightness is a measure of the ability of the source to deliver electrons per unit time within some solid angle at any arbitrary distance from the source. This expression assumes conserved emittance from the source to the sample. It provides a rigorous measure of source brightness for the highest spatial resolution possible within the constraint of negligible space charge effects. For time-resolved measurements, the peak current density has to be pushed to the limit in which there is a trade off in spatial resolution and time resolution needed to address the problem of interest. In general, space charge effects are highly nonlinear and quickly destroy spatial resolution such that there will be a maximum beam current density for a given spatial resolution that depends explicity on propagation distance, as shown in Figure 4. In this case, it is rather obvious that the highest source brightness prior to the onset of space charge aberrations on the spatial resolution is obtained by moving the sample as close to the source as possible. The problem then reduces to eq 3 with respect to the required spatial resolution. The remarkable finding from the theoretical modeling, and demonstrated experimentally, is that with typical transverse photoemission energies (fwhm) of $0.2 \mathrm{eV}$, the transverse coherence length approaches $1 \mathrm{~nm}$ with approximately 1:1 imaging of source to the sample, with sufficient electrons for single shot structure determination. The compact gun design gives this directly with no interverning optics for $100 \mu \mathrm{m}$ beam parameters. This coherence length is sufficient to atomically resolve nearly all chemical systems of interest with single shot capabilities. Even processes involving lattice reorganization associated with electron transfer fall within this parameter space. With relatively minor changes in design for sample positioning, it is now possible to place the sample effectively right at the extraction anode $\mathrm{e}^{49-51}$ for the maximum possible bunch charge density prior to the onset of space charge broadening on the spatial resolution. This is truly an ultrabright electron source in that the imaging is conducted at effectively the highest possible, space charge limited, current density. With the beam parameters at the source, the initial photoemission generated electron distribution appears as a "pancake" distribution $\left(\sigma_{\text {source }} \sim 100 \mu \mathrm{m}, \sigma_{z} \sim 10 \mu \mathrm{m}\right.$ re: Figure 4$)$ such that the dominant space charge effect is longitudinal pulse broadening without significant transverse growth in the emittance. Even at the space charge limit for minimal reduction in transverse coherence, the pulse broadening is only a factor of 2-3 from the pulse at birth (see Figure 4, $t=0.2 \mathrm{~ns}$, which corresponds to $2 \mathrm{~cm}$ from the extraction anode). This longitudinal pulse broadening is a minor reduction from the highest possible current density for a given spatial resolution. The positioning of the sample in close proximity to the extraction anode limits pulse broadening to just the space charge effects within the extraction field, with $<100$ fs pulse durations now possible.

The last vestige of pulse broadening can be removed by exploiting the extraordinary linear chirped nature of the pulses after suitable propagation, as first pointed out by the work shown in Figure 4. This chirp can readily be compensated to recompress the pulse at the sample position using a number of possible dispersive electron optics. The use of reflectron designs was first proposed for compensation of the initial energy spread ${ }^{55}$ and could equally be used for compensation of high bunch charge space charge effects. Another elegant method involves the use of a half cycle rf cavity to temporally compress the pulse. ${ }^{56}$ In principle, the longitudinal space charge effects can be completely removed to regenerate the initial electron pulse duration at birth, which mimics the laser pulse duration for photoinjection. Pulses as short as 10 fs or less are possible, within the constraints of the laser pulse bandwidth and acceptable transverse emittance growth. However, this approach requires nontrivial $\mathrm{rf}$ synchronization with low level rf feedback and passive stabilization of temperature and humidity to avoid drift of the $t=0$ position, which translates to a loss of time resolution. ${ }^{57}$ Time-stamping methods have been developed to help mitigate the jitter problem, which have enabled $30 \mathrm{fs}$ time resolution with adequately short pulses. ${ }^{58}$ The rf pulse compression methods give an approximate factor of 3-10 increase in electron bunch density over the first generation compact gun design for a given transverse coherence at the sample position. For the $100 \mathrm{fs}$ time resolution needed to capture chemical processes, the latest generation compact gun design is now on par with even pulse compression methods for source brightness at the sample position and is no more difficult to use in probing structural dynamics than conventional alloptical pump-probe experiments. The only difference now is the relatively minor pulse broadening in the extraction zone, which can be compensated with pulse compression methods.

The problem comes in scaling femtosecond electron diffraction to study systems with larger unit cells and scaling to higher complexity. The most important relation with respect to scaling to more complex systems is eq 3. It needs to be realized that the transverse coherence length (spatial resolution) is completely determined by the transverse energy distribution of the photoemitted electrons. In terms of increasing the source brightness, one can only approach the space charge limit to the onset of transverse emittance growth with increasing peak current density. The nonlinear nature of space charge effects creates a well-defined threshold for the maximum bunch charge density for any given transverse coherence. Only in the case of perfect ellipsoid charge distributions is it possible to correct the distribution for space charge induced beam divergence, and this has not been realized to date. If we wish to increase the transverse coherence to 10 $\mathrm{nm}$, assuming a transverse energy spread of $0.2 \mathrm{eV}$ (and $\sigma_{\text {sample }}$ $=100 \mu \mathrm{m})$, from eq 3 , we see that the spot size at the photocathode would have to be decreased by 10 relative the sample spot size, which is fixed by the available area of the sample. Thus, in order to increase the spatial resolution to the $10 \mathrm{~nm}$ scale, we would have to reduce the number of electrons per pulse by a factor of 100 relative to the $L_{T}=1 \mathrm{~nm}$ beam parameters with bunch charge density at the space charge limit. 

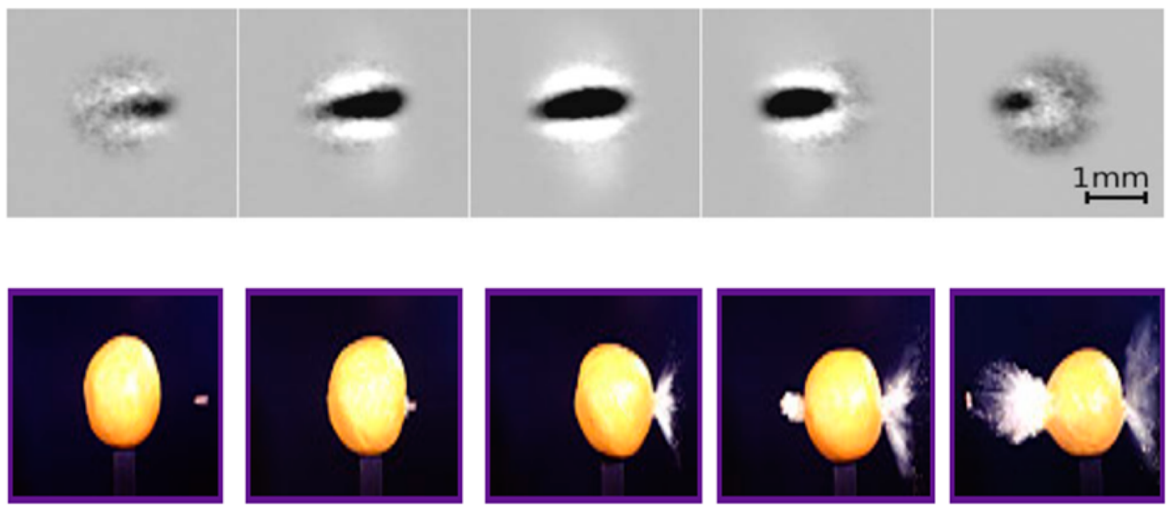

Figure 5. Characterizing Electron Pulses. Top panel shows the detection of an electron pulse on a phosphor screen. The dark "bullet" passing through the electron pulse is the laser excitation pulse, which deflects electrons through the pondermotive force arising from the field gradient of the excitation pulse. Reproduced with permission from ref 53. Copyright 2006 Optical Society of America. (See Movie S4.) The use of grating geometries reduced pulse requirements to microjoule levels. These methods allowed full validation of the electron pulse temporal characteristics. The bottom panel is to give an impression of the enormously fast time resolution. This is catching a bullet in flight; where in the electron case (top), it is catching a laser pulse in flight. Stroboscopic images courtesy of Andrew Davidhazy RIT, http://people.rit.edu/andpph/).

This loss in peak current density to get the desired spatial resolution is not a tenable solution in general. Only in cases where systems are reversible (or using $100 \times$ more sample) can this compromise be made. The best solution is to adopt a different photocathode source with lower emittance. Recently, there have been tremendous strides made in developing new trialkali metal photocathodes with reported energy spreads as low as $20 \mathrm{meV}{ }^{59}$ This is the factor of 10 lower emittance needed to achieve transverse coherence lengths of $10 \mathrm{~nm}$ with the highest possible bunch charge densities at the sample position for the optimal $100 \mu \mathrm{m}$ beam parameters. This new source technology is being implemented and will allow scaling femtosecond electron diffraction studies to cover all conceivable space-time scales relevant to chemistry and its connection to the structure-function of biological systems. Similarly ultracold electron sources have been proposed to scale to even larger coherence lengths with electron temperatures reported as low as $10 \mathrm{~K}(1 \mathrm{meV})$, which would in priniciple extend spatial resolution well beyond $10 \mathrm{~nm}^{60}$

It needs to be fully appreciated that in going to larger length scales in spatial resolution, there is a commensurate requirement for more electrons to resolve the details. This scales approximately quadratically with the unit cell size, assuming constant atom density. As a general rule of thumb from the experiment, $10^{4}-10^{5}$ electrons per pulse are needed for unit cells of $\sim 1 \mathrm{~nm}$ and $10^{6}-10^{7}$ electrons per pulse for unit cells between 3 and $10 \mathrm{~nm}$, assuming sufficient transverse coherence and optimal sample conditions for diffraction. The peak current density at the onset of unacceptable emittance growth due to space charge effects is independent to this consideration. It depends solely on bunch charge density, and as stated above, the magnitude of the space charge effect increases with the propagation distance from the source to the sample. Even with the new low emittance photocathodes and ultracold atom sources, it will not be possible to overcome this electron bunch density limit. The lower emittance of these new sources inceases the spatial resolution but does not change the critical charge density that leads to explosive coulomb growth in emittance. For $100 \mu \mathrm{m}$ beam parameters and $100 \mathrm{fs}$ e pulse durations, very rapid increase in emittance growth occurs at $\sim 10^{5}$ electrons/pulse, as a useful metric for the space charge threshold. Taking $100 \mu \mathrm{m}$ as the maximum value for $\sigma_{\text {sample }}$ due to sample limitations, and a desired e pulse duration of $100 \mathrm{fs}$, the needed total integrated electron numbers must come from either using $10-100 \times$ more sample or using at least partially reversible systems capable of $10-100 \times$ sampling events per excited region to give the required $10^{6}-10^{7}$ integrated signal to achieve sufficient SNR to invert the diffraction pattern to real space. This point must be taken into account in the discussion of low-emittance sources and scaling atomically resolved structural dynamics to more complex systems. It is an imaging problem in which the total detected diffracted electrons at each time point must provide enough information to invert to structures.

As will be seen below, the above estimation of the needed number of electrons for this purpose can be dramatically reduced by exploiting recent advances in electron detectors and the fact that we know the initial structures and conserved features. This additional information helps both in phasing and in reducing the number of detected electrons needed to determine the structure. This advance in image reconstruction and recent low emittance sources will make it possible to achieve single shot structure determination for systems even as large as proteins. In this event, the compact electron gun concept or pulse compression methods are capable of achieving single shot conditions for a given spatial resolution up to systems with unit cells as large as proteins, operating at the maximum space charge limited current. It is in this sense that these sources are ultrabright.

As a final comment on source technology, it is possible to push the peak bunch current density higher by using rf acceleration fields rather than the dc extraction fields discussed above. The oscillating electric field polarity prevents charge build up such that it is possible to increase the extraction field to the dielectric breakdown of the photocathode material or an order of magnitude larger than dc fields. This imparts a small chirp from the rf itself, which with a second rebunching rf cavity can be used to compress electron pulses to less than 1 fs. ${ }^{61}$ This concept is best-suited for relativistic electron energies $(>1$ $\mathrm{MeV})$ where the higher electron velocity $(\sim \mathrm{c})$ enables a longer region of constant compression to place the sample. Thus, it is possible to increase electron source brightness by another factor of 10 by going to relativistic pulse compression. Providing rf time jitter problems can be solved, this pulse 
duration is short enough to open the prospect of imaging electron dynamics (electron density changes in structure factors, vide infra).

From the above discussion, it should be apparent that the definition of reduced brightness is not a sufficient metric for comparing sources for time-resolved studies. A source with a much larger formal reduced brightness may not have the required pulse duration to resolve the dynamics of interest, and similarly, a very short electron pulse may not have the required bunch charge to have sufficient SNR to sufficiently resolve the diffraction pattern. Eq 2 is fine for static imaging in which one is only considering spatial resolution issues of the source. For time-resolved structural studies, a single parameter is insufficient information. We introduce here the term temporal brightness, which we hope will be adopted for which the bunch charge, electron pulse duration, and transverse coherence length should be stated to enable a fair comparison of experimental conditions. These are interdependent parameters. One needs information on all three parameters as this is an imaging problem (intensity and spatial resolution) in the time domain (pulse duration).

To date, the problem in fully characterizing the electron source lies principally with a determination of the electron pulse duration and absolute timing with respect to the laser excitation pulse. Information on the transverse coherence can be reasonably well-approximated and validated through $z$ scans of the beam diameter to determine the beam divergence and from diffraction quality from reference samples. It is the effective temporal resolution of the source that has been most problematic as there is no detector fast enough to provide this information. A known response function must be used to characterize both the electron pulse duration and the convolution to the excitation pulse, which includes any timing jitter between the excitation and probe pulses. In this respect, another important technological development, apart from source brightness, was the characterization of the electron pulses and the $t=0$ position. This measurement problem has been solved with the recent development of pondermotive scattering methods that gives an absolute time resolution characterization to 10 fs accuracy ${ }^{53,62,63}$ (see Figure 5 to see the dramatic light-matter interaction). There are also various streak camera measurements ${ }^{64-66}$ and plasma sources for dynamic ebeam deflection ${ }^{67-69}$ that in combination provide convenient means to calibrate the time resolution below 100 fs. This last feature in relation to characterizing the pulse duration and absolute timing was critical to establishing the overall time resolution. It is now possible to quantitatively compare different sources in terms of temporal brightness in a meaningful way.

To better calibrate the reader to the significance of the technological developments giving rise to the dramatic increase in temporal brightness for electron sources, it is useful to compare to X-ray sources that are more routinely used for molecular structure determination. Given the factor of $10^{6}$ larger scattering cross section of electrons relative to X-rays for the relevant energy ranges, and the need to keep samples thin enough to avoid peak power multiphoton ionization artifacts, this source technology approaches that of X-ray free electron lasers (XFELs) in terms of detected diffraction. Electron guns are tabletop sources of enormous effective brightness. The image and contrast quality for the differential detection of structural changes also depends on source stability. Typical electron sources are orders of magnitude more stable than XFELs and correspondingly need far less sample for resolving structural changes. Specifically, XFELs are self-amplified spontaneous emission (SASE) sources and as such have $100 \%$ shot to shot variability. Averaged over minute time intervals (30-100 Hz operation), the amplitude instability is $5-12 \% \mathrm{rms}$ and wavelength instability is $0.1 \% \mathrm{rms}$ for $0.1 \%$ energy bandwidth, ${ }^{70}$ which affects the degree of partials in the diffraction. This stability is to be compared to electron sources, which replicate the amplitude stability of the solid state laser used for photoinjection, which is typically $0.1 \% \mathrm{rms}$ or better for similar integration times and energy stability corresponding to less than $10^{-5}$ variation. The inherently shorter wavelength of electrons also provides much higher intrinsic spatial resolution than the typical $10 \mathrm{kV}$-range $\mathrm{X}$-rays. The down side is that the sample preparation is much more involved than for X-ray sources. Samples must be on the order of 100-200 $\mathrm{nm}$ thick or less (sample $\mathrm{Z}$ and electron energy dependent) to avoid multiple scattering effects on the diffraction ${ }^{71}$ and must have sufficient surface area to enable collection of the number of desired time points to resolve the dynamics of interest. This difference is a major advantage for XFELs, particularly for the study of biological systems where the protocols for making large area, $100 \mathrm{~nm}$ thin, crystals has not been developed as yet for electrons. Currently, XFELs are the only source capable of atomically resolving dynamics in biological systems ${ }^{4,72,73}$ for which there are a number of key questions related to the coupling of chemistry to biological functions. ${ }^{74,42,4}$ However, there is a large class of systems amenable to study with electron probes, and the current source brightness is sufficient for biological systems (as discussed above and shown in section 4.2). The main limitation is sample preparation. With the advent of nanotechnology and a long-standing effort in the electron microscopy community to provide sufficiently thin samples for electron probes, ${ }^{71}$ the problem of sample preparation can be managed. In this respect, the machine physics for creating and characterizing sufficiently bright electron sources for atomically resolving reaction dynamics has been solved. This advance led to the first atomic movies of structural dynamics with sufficient diffraction orders to resolve the key relative atomic motions directing the structural transition on time scales faster than collisional processes could blur out the details. ${ }^{28}$ It now all comes down to identifying samples to systematically probe different chemical processes. The key enabling feature to get to the point that the sample is the main experimental challenge (as it should be) was the development of high brightness electron sources.

At the opposite end of the spectrum of source brightness, it should also be mentioned that the electron space charge problem can also be solved by going to very low electron density bunches with conventional electron gun/propagation lengths used in TEMs, or the prior state of the art gas phase electron diffraction setups. ${ }^{75-77}$ The space charge limitation in time resolution has to be carefully separated from the overall requirements for image reconstruction, as discussed above. In the limit of so-called single electron pulses, ${ }^{78,79}$ space charge effects can be completely eliminated. This concept has been promoted as the ultimate solution to temporal resolution, and its technical merits need to be discussed. This assertion does not take into consideration all the details of image reconstruction and the minimum dynamic range needed for inverting diffraction to real space images. The experiment is not a simple single parameter integration, in which case the arguments presented would be correct. There is, however, one major advantage of single electron sources in that the spatial 
resolution, in principle if sufficient SNR could be achieved, can be orders of magnitude larger than planar photocathodes. Inspection of eq 3 shows that the longitudinal transverse coherence can be made arbitrarily large by going to extremely small source sizes (e.g., a few nanometers with nanotips). With single electrons, the source size can be made very small to achieve large transverse coherences. The challenge is sufficient sampling to construct the image. To illustrate the limitation of the single electron approach, if we consider a diffraction pattern requiring $10^{6}$ scattered electrons (assuming 100\% detector efficiency) for a system with a unit cell in the 3-10 nm range (e.g., a protein system) to modestly exploit the higher spatial coherence of such sources then the total number of excitation and probe sampling events would have to be on the order of $10^{8}$ (to have 0.01 electrons per photoinjection pulse to ensure single pulse conditions and no loss of spatial-temporal coherence from a $<10 \mu \mathrm{m}$ source size). For real space imaging, the spatial resolution for a given SNR is directly connected to the number of electrons and approaches even more unrealistic numbers of excitation events (i.e., $10^{15}$ for $\AA$ or atomic resolution) for sufficient image contrast $(\mathrm{SNR}=100) .{ }^{80}$ Previous discussions of femtosecond time resolution with $\AA$ spatial resolution have completely neglected this critical detail. ${ }^{77,81}$ The overlooked problem is that of photoreversibility (vide supra). At the present time, there are no known samples undergoing chemical reactions that can sustain up to $>10^{6}$ photocyles under fully reversible conditions as required by this method, even in the limit of $1 \mathrm{e} /$ photoinjection pulse. Recall sample preparation is the main limitation, and single electron approaches will require orders of magnitude more sample than sources capable of single shot atomic resolution. In principle, fully reversible systems can be found, and there has been some very promising work in relation to photoexcitation cycling between reactant and product states ("recover before destroy"). ${ }^{82}$ This set of reversible samples will always be greatly reduced compared to possible studies with high brightness electron sources. However, the real problem will be thermal. Even in the limit of fully reversible systems, the time scale for complete thermal relaxation between laser shots to avoid accumulating heating artifacts and reproducibility will limit sample repetition rates to $100 \mathrm{~Hz}$ time scales, as will the time required for physical exchange of the sample. ${ }^{36}$ These seemingly trivial technical details make the required time and amount of sample challenging to the point of being impractable for the single electron approach for all but gas phase photochemistry. More to the point, there is no reason to use single electron pulse sources for structural dynamics, as per the discussion above in terms of relevant time scales. High brightness sources have achieved the needed space-time resolution to capture the relevant dynamics and factors of $10^{6}-10^{8}$ difference in signal acquisition time, and sample requirements cannot be overlooked. To give another perspective to drive this point home, the prospect of using single electron sources for studying reaction dynamics is the same as using single photon sources for femtosecond spectroscopy. The ability to study femtosecond dynamics was made possible through the introduction of the laser to provide sufficient photon flux to measure small changes in optical properties to correlate to the quantum state dynamics. In like fashion, it would be possible to collect femtosecond timeresolved spectra with single photon sources, but it would take $10^{8}$ longer in conventional pump-probe methods (assuming typical $100 \mathrm{pj}$ probe pulse energies) to achieve the same integrated photon flux for the signal, discounting the much smaller SNR subject to the higher noise at long data collection times. Lasers are high brightness sources, which made these measurements possible. The same consideration holds for electrons. To date, there have been no atomically resolved chemical dynamics exploiting the single electron approach. It all comes down to sample, and only in the limit there were spatial or temporal conditions that could not be met with high brightness electron sources would it make sense to use single electrons for atomically resolved reaction dynamics.

There are, however, certain domains where single electron sources have a major advantage. In this respect, an important case has been made for the study of attosecond dynamics to spatially map electron dynamics using X-rays. ${ }^{83}$ Electrons could be equally used, and single electron pulse sources are more capable of attosecond time resolution for following electron dynamics or problems in solid state physics involved highly correlated motions over 10-100 nm length scales, that tend to be fully reversible, to more fully exploit the intrinsically high spatial coherence of such sources.

The comparison between single electron sources and ultrabright electron sources highlights that the problem of atomically resolving chemical reaction dynamics is really a problem in imaging at the fastest possible framing rates due to sample limitations. The source must not only have sufficient spatial resolution but also brightness and stability for the highest signal-to-noise ratio in obtaining maximum contrast and dynamic range to reconstruct an atomic movie, just like conventional movie cameras. The analogy holds.

\section{Toward a Reaction Mode Basis for Conceptualizing Chemistry}

The above discussion highlights the technical advances made to attain the needed spatial-temporal resolution and most importantly brightness to achieve atomically resolved reaction dynamics. From a chemistry perspective, this new experimental methodology will enable a direct observation of the key reaction modes. Put in another way, these developments enable a determination of the appropriate coordinate space leading to seams in complex potential energy surfaces, the very pinholes in nuclear configuration space leading to the lowest barrier pathways giving rise to the reaction. It needs to be emphasized that we cannot infer these short-lived transition state structures from static structures. The very fact that there is very strong mode coupling in the highly anharmonic region of the barrier crossing region makes it difficult or even impossible to know a priori which modes will yield these seams or their degree of coupling. Our current level of theory is incapable of sufficiently sampling nuclear configurations, save for few-atom systems, to map these motions. Computations do not give sufficiently accurate potential energy surfaces and reaction forces at the critical point for the high-dimensional problems of interest in chemistry. Thus, we need to conjecture about the structure of the transition state. As an important case in point, this problem is amplified by the current efforts to resolve the water splitting structure of PSII that involves a unique arrangement of $\mathrm{Mn}$ and $\mathrm{Ca}$ ions poised after a 4-photon process to generate an intermediate (S4) structure. ${ }^{84,85}$ There is currently no synthetic analogue that can rival PSII. We do not have a detailed understanding of the structure of the barrier crossing region that enables water splitting, which would be a major step forward in the development of alternative energy sources. The problem in conceptualizing transition state structures is akin to 
finding a way from one valley to another in a very rugged mountainous region during an earthquake in which the very mountains and terrain are changing as one moves along the landscape: suddenly an opening appears and the shaking drives you to a more stable valley. Without detailed knowledge of how fluctuations (shaking, in this analogy) affect the potential energy landscape one would be hard pressed to guess where and when an opening or pathway to the next valley would occur. Given the enormous number of possible configurations for molecular systems, this process is best described within a free energy landscape to take into account the entropic terms in the reaction. Even so, the motions involved lead to a common product state or molecular structures such that the key motions at the critical point must be self-similar. This point is reinforced by the very fact that generalized reaction mechanisms can be applied to a huge pallet of molecular systems with nearly arbitrary scaling in complexity ( $N$ degrees of freedom). ${ }^{18} \mathrm{We}$ are looking for common key reaction modes that reflect the anharmonic coupling between spatially more delocalized modes that are otherwise well-approximated by normal modes.

In the past, there has been a tendency to dissect reaction mechanisms into specific details unique to a particular system. This approach misses the above-discussed generalities of chemistry that reduce the dimensionality of the most complex $N$ body problems to a few key modes. The ability to atomically resolve reaction dynamics gives us the tools to directly observe this reduction in dimensionality during barrier crossings and to search for these generalities. It should be possible to construct a localized basis for understanding reaction mechanisms, much like normal modes within a harmonic approximation for the potential energy surface helps to understand equilibrium fluctuations. We are now able to directly observe the far from equilibrium, highly anharmonic, local modes leading to chemical reactions. There are critical reaction volumes within this localized basis that provide sufficient thermal fluctuations to drive such motions. The goal is to systematically observe different types of reactions in order to develop a reaction-mode basis for understanding chemistry.

The major technical obstacle to this objective is the limited number of systems that can be optically triggered under barrierless conditions to project out the reaction modes. In this regard, there needs to be a marriage between theory and experiment in which a few important model systems are used to refine the time-dependent $a b$ initio methods being developed for a first-principles understanding of chemistry. Interestingly, there has been a convergence of theoretical and experimental methods. New theoretical methods are now approaching the ability to handle problems up to several hundred atoms ${ }^{86}$ with sufficient accuracy in the reaction energetics to directly compare to the atomically resolved reaction dynamics. On the experimental front, the signal-to-noise ratio and space-time resolution achieved are now at the point where we can rigorously test these theories. The greatest legacy of atomically resolved reaction dynamics will likely be to refine the next generation theoretical tools that will allow an extension to all chemistry. The great hope for atomically resolved reaction dynamics is to combine the experimental proving ground with theory to provide an extensive reaction mode basis for understanding different chemical reaction mechanisms. The experiments will help develop the theory for projecting these motions to give good a priori approximations to transition state structures to rationally gain control of barrier heights. This insight will represent a significant advance over "arrow pushing", yet still provide a conceptually simple means to guide synthetic strategies. At this point, we will have properly identified the physics that enables chemistry to be a transferrable concept that is robust to scaling in complexity. One can expect that chemistry will then make the transition to mesoscale molecular constructs rivaling the scale and complexity of biological systems.

Chemistry is fundamentally a study of structural dynamics, whether one discusses synthetic targets or physically based methods for the expressed purpose to control the interconversion of matter from one form/property to another. We now have the tools to observe chemistry in action at the atomic level. This review is intended to provide an overview of this emerging field and how it will reshape our understanding of chemistry, and, by extension, our understanding of biological functions.

\section{THEORY ELEMENTS}

Theory is important to both the understanding of the atomically resolved reaction dynamics and the reconstruction of the time-resolved structural dynamics from the diffracted signal in going from reciprocal space to real space. This section provides the background information to appreciate the important role of theory in this class of experiments.

\subsection{Phototriggered Reaction Dynamics: Connection to Thermally Sampled Reaction Coordinates}

The statistics for the thermally sampled barrier are such that, for the barrier heights on the order of $1 \mathrm{eV}(>.5 \mathrm{eV})$ within the time interval of about $100 \mathrm{fs}$ needed to resolve atomic motions, less than 1 molecule in $10^{8}$ would be undergoing a barrier crossing event. Moreover, there is no means to synchronize such events to a detection method, as the initial random thermal sampling of the critical point would be a highly distributed function. It is simply not possible to atomically resolve thermally driven reactions along ground state surfaces. As discussed above, perturbative methods using pump-probe protocols are needed in which a femtosecond laser excitation pulse is used to prepare the system on a reactive excited state surface. The ensuing photochemistry must occur under barrierless conditions to isolate the correlated motions leading the system through a barrier crossing region. If there is an excited state barrier to the reaction, one again is confronted with uncorrelated events involving a very small fraction of the system undergoing barrier crossing within the relevant barrier crossing time. The problem would not be so different from the thermally sampled ground state problem in which the crossing time is much shorter than the buildup of the transient intermediates to the final product ground state. As in the ground state, the much larger background of uncorrelated motions obscures the key motions leading the system through the barrier crossing region. The details of the key motions and reaction forces are lost. More specifically, without a welldefined time origin for all the relevant motions, it is not possible to follow the time-dependent changes in atomic position and corresponding velocities directly related to the reaction forces. In the case of an excited state barrier, one can always assign intermediate structures from either optical data or infrared/Raman studies of reactive intermediates such that little new information is gained. Systems with significant excited state barriers do not fully warrant the use and complexity of timeresolved diffraction methods. Instead, systems that undergo photochemical processes under barrierless or near barrierless 


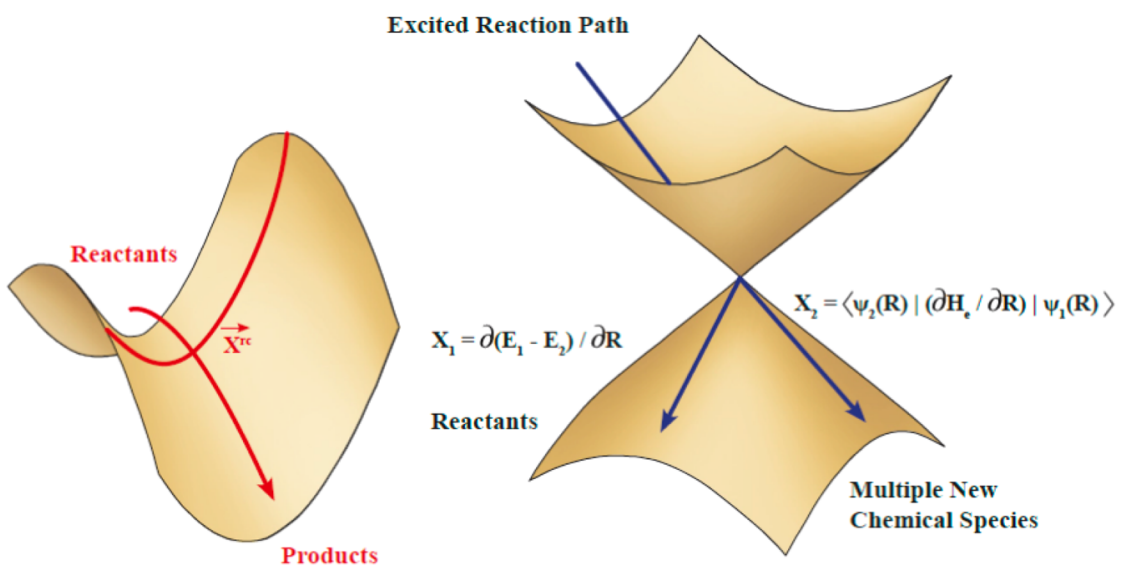

Figure 6. Comparison of reaction paths through a thermally sampled transition state vs optical preparation on an excited state surface involving a conical intersection. The initially prepared excited state relaxes to similar nuclear configurations on the ground state potential. Adapted with permission from ref 91 . Copyright 2014 Springer-Verlag.

conditions should be studied. The problem then reduces to connecting the reaction forces observed under far-fromequilibrium positions in nuclear coordinate space along the excited state reaction coordinate to the thermally sampled ground state process. This problem is very similar to that encountered in molecular dynamics (MD) treatments of reaction dynamics, even within a classical description. ${ }^{87}$ The key point to be made is that as long as the system/bath is large enough such that the atomic motions are occurring near linear response limits, the key reaction modes and net amplitudes will be similar to the thermal case. Put in another way, as long as the curvature of the excited state potential matches and crosses onto the ground state potential, the force distribution and action will be similar. This point can be understood by simply considering time reversal of the atomic displacements once the system crosses onto the ground state product electronic surface.

The assumption here is that the excited state surface crosses through the same point in nuclear configurations as ground state processes (i.e., there is a common crossing point for the thermal and photoexcited reaction involving the formation of the same final product state). This assumption holds in most cases except when the photoexcited surface lies energetically above multiple barriers to competing processes and relaxes to a distribution of photoproducts. In this case, the reaction dynamics need to be treated as a uniquely excited state process. The information content is still rigorous with respect to comparing to time-dependent $a b$ initio theory as part of the overall goal to develop the toolset to understand chemistry up to arbitrarily large complexity. Indeed, if the separation of the different reaction channels can be made, even in this limit, it should still be possible to connect the reaction modes involved in the thermally sampled region to sufficiently robust theoretical methods.

With regard to comparison to theory, similar considerations hold with respect to requiring an impulsive perturbation to prepare the system. It is computationally too intensive to isolate rare events occurring within a thermally equilibrated ensemble. The use of a photoprepared excited state, as a means to synchronize the reaction dynamics, is as much an essential device for time-dependent $a b$ initio calculations as it is for the experiments.

The depiction of the reaction dynamics can be conceptualized using the adiabatic potential energy surfaces (APES) of the ground and excited states of the molecular systems ${ }^{88-90}$ as shown in Figure 6.

A reaction on the ground state surface with only one reactive coordinate implies a saddle point in the multidimensional potential energy surface. Along that coordinate, there is a strong anharmonicity as the system approaches the transition state between reactants and products. Thermal energy, which initially may be in other vibrational coordinates of the complex, $\mathrm{N}$-dimensinal surface, must be channeled to the anharmonic reaction coordinate in order for the reaction to proceed.

Figure 6 also shows the equivalent photoinduced reaction involving the excited state surface. Many reactions proceed under barrierless conditions and involve conical intersections (CIs) that facilitate the transition from the excited state to the ground state surface. ${ }^{92}$ The CIs are points or seams in the multidimensional potential energy surfaces where the BornOppenheimer approximation breaks down (i.e., where nuclear and electron motions are highly coupled). The molecular system typically proceeds down the sloping potential and through the CI very quickly, so that the reactions occur on femtosecond time scales. Because of the fast motions and complicated nature of the $\mathrm{CI}$, dynamical processes are difficult to observe spectroscopically. ${ }^{93}$ Additionally, it is difficult to differentiate the generation of reaction products from transitions through CIs from the statistical processes and to disentangle the reaction paths when multiple CIs are involved.

Time-resolved electron diffraction (TRED) $)^{94-96}$ has been pointed out to hold great potential for directly determining the reaction modes, and with correlation to the observed time scale can unambiguously assign passage through a CI. To date there is only one example with sufficient simultaneous space-time resolution to distinguish $\mathrm{CI}$ mechanisms from alternative pathways directly from experiment (vide infra). ${ }^{35}$ The current approach largely follows spectroscopic approaches using a combination of theory and observable dynamics to infer the involvement of a CI. The use of electron probes in TRED, or $\mathrm{X}$-rays in time-resolved X-ray diffraction (TRXD), even without sufficient spatial or time resolution do, however, provide additional structural information on intermediate states, which helps to constrain theory to increased confidence in the assignment, as will be dicussed below. 


\subsection{Conical Intersections-Conceptualizing Reaction Coordinates}

Despite the unresolved questions surrounding the role of CIs in excited state processes, they provide a framework for understanding the reduced dimensionality in barrier regions. Moreover, for small systems $(N<10)$, the concept of a CI can be rigorously derived, and such systems form useful models. In addition, the presence of a CI has been predicted to give rise to purely quantum mechanical effects such as a Berry phase effect $^{97}$ in the quantum yield for a particular product state as a function of spatial and momentum coordinates. The idea is that a phase shift of the time-dependent wave function at the crossing point leads to destructive interference that reduces the reaction probability for a particular region in nuclear configuration-momentum space, even though the motions are still the primary modes driving the reaction. It is an unusually "spooky" quantum effect that is of general relevance in photochemistry with respect to directing structural transitions. Given the $10-100 \mathrm{fs}$ times scales involved, only femtosecond pump-probe methods can reach the needed time-resolution and correlation to the quantum state evolution. This phenomenon has yet to be observed experimentally as the transition dipole for probing this region near the CI vanishes. However, the electron diffraction observable does not depend on transition dipoles. Therefore, femtosecond electron diffraction could provide a definitive test for the quantum nature of a particular photochemical reaction, as an acid test for describing nonradiative channels in the formalism of CIs.

In small molecules, the existence of CIs is related to the number of close-lying electronic states and their respective symmetries. An important question is how this concept translates to large molecules. The effective potential energy surface frequently considers only two reaction modes, the tuning and coupling modes. In large molecules, there will exist competing pathways and more complex potential energy surfaces than assumed in the conventional CI description. This point is illustrated in Figure 7.

Originally predicted in 1929 by von Neumann and Wigner, ${ }^{99}$ CIs result from electronic degeneracies. In diatomic molecules, the noncrossing rule states that two electronic states of the same symmetry cannot cross. Teller realized that in polyatomic molecules the possibility of surface crossings depends on the symmetry of the molecule: crossings are allowed but only in a space of reduced dimensionality of $(n-2)$, where $n$ is the number of vibrational degrees of freedom, which is given by $3 N$ -6 for a nonlinear molecule of $\mathrm{N}$ atoms. ${ }^{100}$ According to von Neuman and Wigner, a degeneracy can arise when two degrees of freedom are varied independently. The gradient difference vector $\left(\mathbf{x}_{1}\right)$, and the derivative coupling vector $\left(\mathbf{x}_{2}\right)$, are defined as ${ }^{101,102}$

$$
\begin{aligned}
& \mathbf{X}_{1}=\partial\left(\mathbf{E}_{1}-\mathbf{E}_{2}\right) / \partial \mathbf{R} \\
& \mathbf{X}_{2}=\left\langle\psi_{\mathbf{2}}(\mathbf{R})\left|\left(\partial \mathbf{H}_{\mathbf{e}} / \partial \mathbf{R}\right)\right| \psi_{\mathbf{1}}(\mathbf{R})\right\rangle
\end{aligned}
$$

where $\mathbf{H}_{e}$ is the electronic Hamiltonian, $\mathbf{E}_{\mathbf{1}}$ and $\mathbf{E}_{\mathbf{2}}$ are the two electronic eigenvalues, and $\mathbf{R}$ is a vector of nuclear Cartesian displacements. The wave functions and $\psi_{1}$ and $\psi_{2}$ are solutions of the electronic Schrödinger equation.

Any displacement of the system along the gradient difference vector or the derivative coupling vector lifts the degeneracy. Therefore, graphing the electronic surfaces as a function of those two vectors reveals the double-coned potential energy surfaces illustrated in Figure 6, right. It is via such "photo-

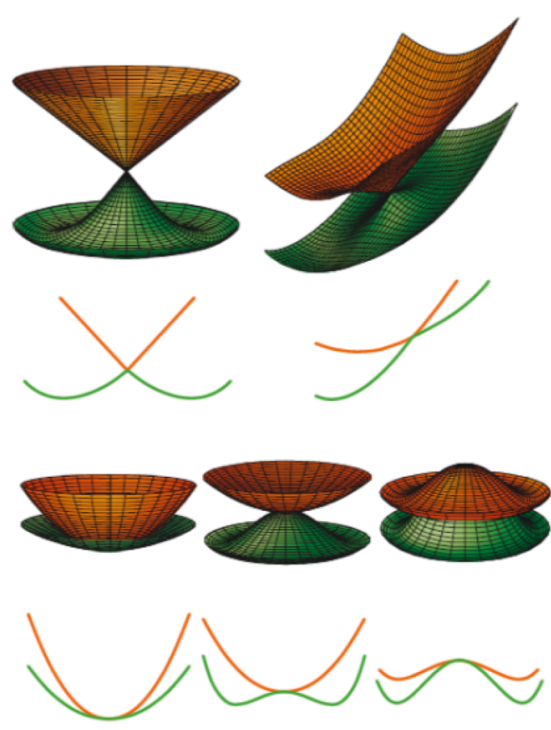

Figure 7. Schematic illustration of conical intersection topologies (top) and Renner-Teller topologies (bottom). A linear vibronic JahnTeller coupling gives rise to a generic, circular cone (top left). In a general vibronic coupling situation with three linear vibronic coupling constants yields a sloped conical intersection (top right). The cone is tilted because the force gradient vectors on both the upper and lower surfaces slope in the same direction. The sloped and peaked topologies affect the dynamics and the outcomes of the photochemical reactions. Renner-Teller types I, II, and III surfaces, which result from only second-order vibronic coupling, are shown on the bottom. These topologies result in the destabilization of both surfaces (type I), stabilization of the lower surface only (type II), and the stabilization of both surfaces (type III). Adapted with permission from ref 98 . Copyright 2010 Elsevier.

chemical funnels" that molecular systems can pass from an excited state to a ground state surface. The important consideration for the reaction is whether the cones are circular or possibly elliptic in shape, which depends on the magnitudes of the $\mathbf{x}_{1}$ and $\mathbf{x}_{2}$ vectors.

For small molecular systems, symmetry imposes the degeneracy that leads to the Jahn-Teller effect. For large molecular systems, many topologies of CIs are possible. Therefore, while in small molecules one can visualize a transition state that connects the reactants to products via a single reaction path along the transition vector $\mathrm{x}^{\mathrm{rc}}$ (Figure 6), this motion is poorly defined for large molecules. Branches of the reaction path in the plane $\mathbf{x}_{1}$ and $\mathbf{x}_{2}$ can connect reactants to two or more photoproducts. Vibrational degrees of freedom outside those two vectors, numbered $3 N-8$ in the $N$ atom molecule, span a multidimensional space in which the $\mathrm{CI}$ consists of an infinite number of points known as a seam. Clearly, the complexity of this space for large moleucles is confounding, and it again raises the question of the reduction in dimensionality that must accompany the chemical reaction.

The position and topology of CIs help to understand the photochemical reaction pathways of small molecules. When both the ground and excited potential energy surfaces have orthogonal gradients near the intersection (such as shown in Figure 6), a peaked CI results. Such a topology enables a very efficient transition from the excited state surface to the ground state surface. On the other hand, very similar gradient vectors near the CI give rise to a sloped CI such as the one in Figure 7, right. Here, the reaction pathway needs to be well-aligned with the $\mathrm{CI}$ in order to allow for facile transition from the upper to 
the lower surface. The $\mathbf{x}_{2}$ vector is zero by symmetry when two surfaces of different spin states cross, resulting in a seam of dimension $3 N-7$. This is the case in intersystem crossing, where the crossing rate depends on the Franck-Condon overlap and resonances between spin manifolds and spin-orbit coupling.

Conical intersections provide the degeneracies to enable transitions between electronic surfaces on the time scale of the vibrational periods coupling the two surfaces, which vary from 10 to $100 \mathrm{fs}$. The energy released by the electronic transition can be in the form of kinetic energy but must quickly be redistributed in the molecule through intramolecular vibrational relation (IVR). For small systems, the mode density may not be sufficiently high, so that the passage to the product state is not fully determined by the period of the strongly damped reaction modes. In large molecules $(N>10)$ in regions of large density of states, IVR occurs within 100 fs to $1 \mathrm{ps}^{11,12,103}$ This meets the above condition for collapsing the wave function after the CI onto a product channel. This relaxation mechanism is important in the photostabilization mechanisms of the primary events in vision. ${ }^{43,104}$ The challenge is to identify a coordinate space of reaction coordinates that reveals CIs involved in the reaction.

\subsection{Recent Advances in Time-Dependent Ab Initio Methods}

The 2D conical intersection captures the essence of the reduction of the dimensionality to a few reacting modes. Another important concept arising from this line of reasoning is the importance of degeneracies in the electronic surfaces that lead to a break down of the BO approximation. The time scale for electronic redistribution within the two electronic surfaces depends inversely on the energy gap between the two surfaces coupled through nuclear fluctuations. There is no longer a separation in time scales between the electronic and nuclear degrees of freedom, and the wave function can no longer be factored into electronic and nuclear terms. The full timedependent Schroedinger equation must be solved in the presence of nuclear fluctuations, for the entire molecular system or reaction complex of interest. The computational cost for this level of theory is too high to be practical at present, and normally a reduced molecular model system of the core reaction site is used to approximate the system. The involvement of the intramolecular and intermolecular baths is generally treated classically in a QM/MM formalism. As the truncated molecular model is an approach to simplify the problem, the bath coupling must also be approximated, normally at the lowest level, assuming linear vibrational coupling.

This area is outside the main focus of this review; however, the inclusion of the current status of theory is needed to illustrate the essential integration of theory and experiment. There is only a small subset of chemical reactions that are amenable to direct experimental observation of real time atomic motions for the reasons discussed above. It is therefore essential to have the atomically resolved dynamics serve as rigorous benchmarks to refine theoretical methods to ultimately enable extension to other areas of chemistry. The experimental observable is precisely the objective of the theoretical methods being developed for understanding reaction dynamics. It is also interesting to note that theory is going through the same challenges as experiments to scale to higher levels of complexity to approach relevant time scales. While the experimentalists need a brighter source, the theoreticians need a "brighter" code. The problem is to handle in a computationally efficient manner the electron correlation effects on reaction dynamics for larger and larger systems. The goal is to approach the scale of current interest in chemistry, without using the $\mathrm{BO}$ approximation to factor the wave function into nuclear and electronic degrees of freedom.

In terms of scaling this level of theory to larger systems, to the point of modeling the photochemistry of biomolecules, perhaps the greatest progress has been in the development and proliferation of single-reference $a b$ initio methods for the calculation of excited states, especially those that take advantage of the resolution-of-the-identity approximation. ${ }^{105-107}$ This latter approximation, also known as density fitting, significantly reduces the computational cost of the calculating four-center, two-electron integrals, leading to an order-of-magnitude speedup of the overall calculation. Quantitatively accurate excitedstate calculations can be carried out for relatively large systems such as molecular clusters and nucleosides. ${ }^{108,109}$ The implementation of nonadiabatic coupling vectors and matrix elements between singlet excited states ${ }^{10,111}$ has enabled the application of some such methods to excited state to excited state internal conversion processes; however, the description of excited state to ground state crossings remains problematic. ${ }^{112}$

An even higher computational efficiency is achieved by semiempirical electronic structure methods adapted to excitedstate calculations, ${ }^{113}$ such as the well-known OM2/MRCI method, ${ }^{114,115}$ though at the cost of parametrization against experimental and/or theoretical reference data. In particular, the OM2/MRCI method has been successfully applied to various photoinduced processes of large organic molecules (see ref 113 and refs therein). It should be pointed out, however, that the range of applicability of this and other semiempirical methods is limited by inherent requirements of prior parametrization and validation against suitable benchmarks for each specific system to be simulated.

Another cornerstone of computational photochemistry is the venerable QM/MM method of Warshel and Levitt, ${ }^{116}$ whereby only the main reaction site of the system is treated using a quantum-mechanical (QM) electronic structure method, while its surroundings are described using a classical molecular mechanics (MM) calculation. Since the computational cost of an $\mathrm{MM}$ calculation is typically very low in comparison to a QM excited-state calculation, the QM/MM method allows the simulation of photochemical reactions in large systems such as biomolecules and crystals, provided that the reaction can be considered as localized within a well-defined and reasonably small, reaction site. As we will see below, this is the correct picture. This approach has been extended by exploiting Ehrenfest's approximation, in which the wave function of the quantum system evolves in response to forces from the classical bath that in turn experiences forces from the mean field of the quantum system. This idea exploits the delocalized nature of quantum states, but the quantum state evolution is not compatible with the localized nature of classical trajectories and typically gets product state distributions wrong. This approximation works well for short times, and great progress has been made using multiple configurations and spawning methods to improve computational efficiency to enable the study of very large systems. ${ }^{117}$ There are also new ideas of how to solve the classical-quantum conundrum through the use of quantum classical path integral methods that do not invoke any approximations, with very promising results to date in terms of 
treating photochemical reactions relevant to optimizing synthetic strategies. ${ }^{118}$ Theory and experiment are converging.

\subsection{Theory of Pump-Probe Electron Diffraction: Inversion} Problem

This section will use an isolated two level molecular system to illustrate the basic principles and the care needed to analyze diffraction data to obtain real space information. The focus is on diffraction as the signal scales as $\mathrm{N}^{\prime 2}$ and is the only tractable means to achieve atomic imaging in real time (vide supra). Even with the amplification from diffraction, we need to push the quantum limits in information extraction from the quantum scattering. This aspect of atomically resolved reaction dynamics is one of the major areas needing significantly greater effort to achieve the ultimate limits in imaging molecular dynamics. Also, it needs to be pointed out that one of the major challenges in image reconstruction using diffraction, or reciprocal space imaging, is the unknown phase relationship of the different diffraction orders. This issue is known as the inversion problem that plagues all diffraction experiments. There have been a number of solutions to the problem using molecular models and some degree of correlation to ascertain accuracy. Without a reference, such as provided in holography, there is no direct means to mathematically uniquely invert directly from reciprocal space to real space. There is always some uncertainty in the model. However, for time-resolved measurements there is additional information in that one knows the initial structure and some details regarding the excited state dynamics. One is not trying to solve a completely unknown structure. As will be detailed below, it is possible in principle with sufficiently high time resolution to perform a tomographic reconstruction. Alternatively, it has just been shown that by knowing the initial structure and conserved bond and bond angles in the product structure there is enough information to solve the inversion problem to arrive at unique convergence to the real space structure (treated below). These are important advances that finally enabled time-resolved diffraction methods to achieve their promise of full atom resolved structural dynamics.

The experiment necessarily involves optical preparation of an excited state to trigger the chemistry. To formulate the problem, consider the free movement of an isolated molecular system in the absence of radiation and external fields. Its movement is completely determined by the time-independent Hamiltonian $\mathbf{H}_{0}$ and described by its initial state, the wave function $\boldsymbol{\Psi}_{0}$. The action of a radiation field (the pump pulse) with an amplitude of $\mathbf{E}(t)$ results in a change of the free movement of the molecule (rotational, vibrational, and electronic degrees of freedom). Moreover, these changes can be controlled by changing the field frequency and (or) by its temporal structure. This opens up an opportunity to control the molecular dynamics and with it the transformation of the molecular system. ${ }^{119,120}$

The Hamiltonian of the molecule in the laser field can be written as

$$
\mathbf{H}=\mathbf{H}_{0}+\mu(r) \mathbf{E}(t)
$$

where $\mu(r)$ is the position-dependent but time-independent dipole moment, while the amplitude of the field $\mathbf{E}(t)$ depends only on time. ${ }^{120}$

For coherent excitation, when the system undergoes a transition from the ground state $(\mathbf{g})$ to the excited state $(\mathbf{e x})$, the evolution of this state is described by a time-dependent Schrödinger equation:

$$
(i h / 2 \pi) \partial \Psi / \partial \mathrm{t}=\mathbf{H} \Psi
$$

and the wave function of the system is described by a vector, indicated by the indexes of the ground and the excited states $\{\mathbf{g}$, ex $\}$, and, accordingly, $\boldsymbol{\Psi}=\left\{\boldsymbol{\Psi}_{\mathrm{g}}, \boldsymbol{\Psi}_{\mathrm{ex}}\right\}$.

The energy of the interaction:

$$
\mathbf{W}(r, t)=-\mu(r) \mathbf{E}(t)
$$

is the main characteristic of the coherent excitation and determines the frequency of oscillation between the ground and the excited states, the Rabi frequency:

$$
\Omega_{\mathbf{R}}(r, t)=2 \pi|\mathbf{W}(r, t)| / h=2 \pi \mu(\mathrm{r})|\mathbf{E}(t)| / h
$$

For the radiation resonantly interacting with a two-level system, the excitation probability is defined as

$$
\mathbf{P}_{\mathbf{e x}}(r, t)=[\sin (1 / 2 A(r, t))]^{2}=(1 / 2)[1-\cos A(r, t)]
$$

where the function $A(r, t)$ is determined as

$$
A(r, t)=\int_{-\infty, t} \Omega_{\mathbf{R}}\left(r, t^{\prime}\right) \mathrm{dt}^{\prime}
$$

Thus, under coherent excitation the normalized population of both the ground and the excited states oscillates between 0 and 1 at the Rabi frequency, defined by eq 9. In particular, the probability of the excitation reaches its maximum at $A(r, t)=$ $(2 n+1) \pi$, where $n$ is an integer number, the multiple of socalled $\pi$-pulses. When averaged over a large number of Rabi cycles, the population of both the ground and the excited states are equal, as in the case of incoherent excitation.

To achieve efficient excitation with optimal and stable probabilities, as compared with both the coherent and the incoherent excitation, it is advantageous to use coherent optical pulses with frequencies that vary slowly with respect to the resonant value (adiabatic passage). ${ }^{120-122}$ There are also other modifications of the adiabatic transition, described in the works of Kosloff et al. ${ }^{123}$ and Bardeen et al. ${ }^{124,125}$ The use of these techniques makes it possible to achieve an efficient and rather selective population transfer between molecular states.

For adiabatic excitation over a large interval of time with some bottleneck state, the population transfer may equal $100 \%$, and it is possible to use the theory described in the studies of Ischenko. ${ }^{94-96,126-128}$ For incoherent excitation on long time scales, it is possible to interpret the experimental data utilizing the model of additive contributions of the ground and the excited states of the molecular ensemble with a known ratio of the populations of the two-level system (i.e., 1:1). Thus, it is also possible to implement that theory, ${ }^{94,127,128}$ with the superposition of two states. For coherent excitation, the same situation occurs, when the duration $\tau_{\mathrm{e}}$ of the diffracting (electron) pulse probing the studied system becomes so great that the relation $\tau_{\mathrm{e}} \gg 2 \pi / \Omega_{R}$ holds. Here, we will consider the coherent excitation and molecular diagnostics by electron pulses of rather short duration to avoid saturation and averaging effects. In the time-resolved diffraction experiment, the recorded signal reflects an integral pattern of the transmission of the ultrashort electron bunch through a target as a function of temporal delay of $\tau_{\mathrm{d}}$ between the exciting laser and the probing electron pulses.

The wave function of the system can be represented as ${ }^{129}$

$$
|\Psi(r, t)\rangle=\sum_{j} C_{j}(t)\left|\Psi_{j}(r, t)\right\rangle
$$


where $C_{j}(t)$ determines the probability amplitude of the state $j$. Then, in the approximation of independent atoms, ${ }^{130}$ the expression for the time-dependent intensity of the electron scattering by the molecular system at some instant of time $t$ can be written as follows:

$$
\begin{aligned}
\mathbf{M}(\mathrm{s}, \mathrm{t})= & \left(I_{0} / R^{2}\right) \operatorname{Re}\left\{\mathrm { g } ( \mathrm { s } ) \sum _ { \mathrm { j } } C _ { \mathrm { j } } ( \mathrm { t } ) * C _ { \mathrm { j } } ( \mathrm { t } ) \left\langle\Psi_{\mathbf{j}}(r, t)\right.\right. \\
& \left.\left.|\exp (\operatorname{isr})| \Psi_{\mathrm{j}}(r, t)\right\rangle\right\}
\end{aligned}
$$

where $j$ denotes the electronic state, $g(s)$ are the functions of the atomic amplitudes and the phases of the scattering, ${ }^{131} R$ the distance from the scattering point to the center of the diffraction pattern at the detector, $s$ the vector of the momentum transfer in the laboratory frame, and $r$ the instantaneous internuclear distance. It is well-known, that the values of $\left|C_{j}(t)\right|^{2}$ reflect the evolution of the population of each state $j$. For a two-level system the wave function $\boldsymbol{\Psi}(r, t)$ may be represented as

$$
\Psi(r, t)=C_{\mathbf{g}}(t) \Psi_{\mathbf{g}}(r, t)+C_{\mathrm{ex}}(t) \Psi_{\mathrm{ex}}(r, t)
$$

where

$$
\begin{aligned}
& \Psi_{\mathrm{g}}(r, t)=\varphi_{\mathbf{g}}(r) \exp \left(-2 \pi i E_{\mathrm{g}} \mathrm{t} / h\right) \\
& \Psi_{\mathbf{e x}}(r, t)=\varphi_{\mathbf{e x}}(r) \exp \left(-2 \pi i E_{\mathbf{e x}} t / h\right)
\end{aligned}
$$

Then, eq 13 can be rewritten as follows:

$$
\begin{aligned}
\mathbf{M}(s, t)= & \left(I_{0} / R^{2}\right) \operatorname{Re}\left\{\mathrm { g } ( s ) \left[\mathbf{M}_{\mathbf{g}}(s, t)+\mathbf{M}_{\mathbf{e x}}(s, t)\right.\right. \\
& \left.\left.+\mathbf{M}_{\mathbf{i n t}}(s, t)\right]\right\}
\end{aligned}
$$

for which

$$
\begin{aligned}
\mathbf{M}_{\mathbf{g}}(s, t)= & C_{\mathrm{g}}(t)^{*} C_{\mathrm{g}}(t)\left\langle\varphi_{\mathbf{g}}(r)|\exp (i \mathbf{s r})| \varphi_{\mathbf{g}}(r)\right\rangle \\
\mathbf{M}_{\mathbf{e x}}(s, t)= & C_{\mathbf{e x}}(t) * C_{\mathbf{e x}}(t)\left\langle\varphi_{\mathbf{e x}}(r)|\exp (i \mathbf{s r})| \varphi_{\mathbf{e x}}(r)\right\rangle \\
\mathbf{M}_{\mathrm{int}}(s, t)= & \left.C_{\mathbf{g}}(t) * C_{\mathbf{e x}}(t) \exp \left[i\left(\omega_{\mathrm{g}}-\omega_{\mathrm{ex}}\right) t\right] \varphi_{\mathrm{g}}(r)|\exp (i \mathbf{s r})| \varphi_{\mathbf{e x}}(r)\right\rangle \\
& \left.+C_{\mathrm{ex}}(t)^{*} C_{\mathrm{g}}(t) \exp \left[i\left(\omega_{\mathrm{ex}}-\omega_{\mathrm{g}}\right) t\right] \varphi_{\mathrm{ex}}(r)|\exp (i \mathbf{s r})| \varphi_{\mathbf{g}}(r)\right\rangle
\end{aligned}
$$

where the angular frequency $\omega_{g(\mathrm{ex})}=2 \pi E_{\mathrm{g}(\mathrm{ex})} / \mathrm{h}$. The first two terms in the eq 17 are the contributions from the ground state (eq 18a) and the excited state (eq 18b), correspondingly. The "interference" term in eq $17, \mathbf{M}_{\text {int }}(s, t)$, gives the contribution to the total intensity of the molecular scattering due to the interaction between the ground and the excited states in the laser field (eq 18c). Importantly, the interference term allows for the determination of the off-diagonal elements of the density matrix during the tomographic reconstruction of the molecular quantum state of the studied system. ${ }^{128}$ It describes qualitatively new information about the molecular system, which can be obtained from the diffraction data by solving the inversion problem, for example by using the theory described in the studies of refs 132 and 133. The tomographic reconstruction can be achieved only when using sufficiently short electron pulses where $\tau_{e}<2 \pi /\left|\omega_{g}-\omega_{e x}\right|$. The required pulse duration to achieve this condition is less than the period of the carrier frequency of the excitation pulse such that abovementioned averaging over time can "wash out" the contributions of the interference term in eq 17 and thereby render impossible the reconstruction.
To illustrate the explicit form of the dependence of the molecular scattering intensity on the parameters of the laser excitation and also on the molecular system parameters, one may take the wave functions used in the approximation of state preparation with ultrashort laser pulses. ${ }^{134}$ This approach explicitly takes into account the dependence of the wave functions on the nuclear coordinates (eqs 14-17) by expressing the ground and excited state wave functions (eqs 15 and 16$)$ as

$$
\begin{aligned}
& \Psi_{\mathrm{g}}(\mathrm{r}, \mathrm{t})=\exp \left[\mathbf{i} \Phi_{\mathrm{g}}(r) t\right]\left\{\cos \left(\Omega_{\mathrm{R}} t\right)+\mathbf{i} \cos \theta \sin \left(\Omega_{\mathrm{R}} t\right)\right\} \Psi_{\mathrm{g}}(r) \\
& \Psi_{\mathrm{ex}}(r, t)=\exp \left[\mathbf{i} \Phi_{\mathrm{ex}}(r) t\right]\left\{\sin \theta \sin \left(\Omega_{\mathrm{R}} t\right)\right\} \Psi_{\mathrm{ex}}(r)
\end{aligned}
$$

where

$$
\begin{aligned}
& \Phi_{\mathrm{g}}(r)=-\omega_{\mathrm{g}}-2 \pi \Delta(r) / h \\
& \Psi_{\mathrm{ex}}(r)=-\left(\omega_{0}+\omega_{\mathrm{g}}\right)-\pi^{2} / h-2 \pi \Delta(r) / h \\
& \Delta(r)=(1 / 2)\left\{V_{\mathrm{ex}}(r)-V_{\mathrm{g}}(r)-h \omega_{0}\right\}
\end{aligned}
$$

and the value of $\boldsymbol{\theta}$ is defined as

$$
\theta=\tan ^{-1}\{|\mathbf{W}(r, t)| / \Delta(r)\}
$$

In eqs $20 \mathrm{~b}$ and $21, \omega_{0}$ is the carrier frequency, $V_{\mathrm{g}}(r)$ and $V_{\text {ex }}(r)$ are the potential energy functions of the ground and the excited states. The wave functions $\boldsymbol{\Psi}_{\mathrm{g}}(r)$ and $\boldsymbol{\Psi}_{\mathrm{ex}}(r)$ are the solutions of the time-independent Schrödinger equation for the ground and the excited states.

The study of eq $18 \mathrm{a}$, eq $18 \mathrm{~b}$, and eq $18 \mathrm{c}$ shows that the total molecular scattering intensity $\mathbf{M}(s, t)$ oscillates with the Rabi frequency $\boldsymbol{\Omega}_{\mathrm{R}}$, together with the populations of the excited and the ground states. It is interesting to note that if the molecular system dissociates in the excited state, the population of the excited state "washes out", causing a complete transition of the population from the ground state to the excited state. In other words, the exciting laser pulse "cuts out" some part from the original ground state population, which is removed through the potential surface of the excited state; the "missing part" at the surface of the ground state is a dynamic "hole". ${ }^{34}$ For short pulses and where $\Delta(r) t<\pi$, the maximum effect is achieved at the point of the resonance, where $\Delta(r)=(1 / 2)\left\{V_{\mathrm{ex}}(r)-V_{\mathrm{g}}(r)\right.$ $\left.-h \omega_{0}\right\}=0$. The change of the carrier frequency $\omega_{0}$ causes a shift in $\Delta(r)$, overriding the position of the resonance, $r=r_{\text {res }}$, where $\Delta\left(r_{\text {res }}\right)=0$. Since this point is at the center of the dynamical "hole", $\omega_{0}$ becomes a very important control parameter. As already mentioned above, the maximum effect of the hole formation is achieved for $\pi$-pulses, when $(1 / 2) \mathbf{W}(r$, $t) t=\pi$; for $(1 / 2) \mathbf{W}(r, t) t=2 \pi$, the dynamic hole is formed again.

Finally, the eqs 18-22) allow us to represent the intensity of the molecular scattering in the following form:

$$
\begin{aligned}
\mathbf{M}_{\mathrm{g}}(s, t)= & \int \mathbf{P}_{\mathrm{vib}, \mathrm{g}}(r, t)\left\{\cos ^{2}\left[\Omega_{\mathrm{R}}(r, t) t\right]\right. \\
& \left.+\cos ^{2}[\theta(r, t)] \sin ^{2}\left[\Omega_{\mathrm{R}}(r, t) t\right]\right\} \exp (i \mathbf{s r}) \mathrm{dr} \\
\mathbf{M}_{\mathbf{e x}}(\mathrm{s}, \mathrm{t})= & \int \mathbf{P}_{\mathrm{vib}, \mathbf{e x}}(r, t) \sin ^{2}[q(r, t)] \sin ^{2}\left[\Omega_{\mathbf{R}}(r, t) t\right] \\
& \times \exp (i \mathbf{s r}) \mathrm{dr}
\end{aligned}
$$


where $\mathbf{P}_{\text {vib },(\text { ex })}(r, t)=\left|\Psi_{\mathrm{g}(\mathrm{ex})}(r, \mathrm{t})\right|^{2}$ are the probability density functions for the ground and the excited molecular states.

For the interference term, we get the following equation:

$$
\begin{aligned}
& \mathbf{M}_{\text {int }}(s, t) \\
& \quad=2 \int \mathbf{P}_{\text {vib,int }}(r, t) \mid \sin [\theta(r, t)] \| \sin \left[\Omega_{\mathbf{R}}(r, t) t\right] \\
& \quad+\left\{\cos ^{2}\left[\theta_{\mathbf{R}}(r, t) t\right]\right. \\
& \left.\quad+\cos ^{2}[\theta(r, t)] \sin ^{2}\left[\Omega_{\mathbf{R}}(r, t) t\right]\right\}^{1 / 2} \\
& \quad \times \sin \left\{\left(\omega_{0}+\pi^{2} / h\right) t+\tan ^{-1}[\cos [\theta(r, t)]\right. \\
& \left.\quad \times\left[\tan \left[\Omega_{\mathbf{R}}(r, t) t\right]\right]\right\} \exp (i \mathbf{s r}) \mathrm{dr}
\end{aligned}
$$

where $\mathbf{P}_{\text {vib,int }}(r, t)=\left\langle\boldsymbol{\Psi}_{\text {ex }}(r, t) \mid \Psi_{\mathrm{g}}(r, t)\right\rangle$. In all the equations, following eq 19a and eq $19 \mathrm{~b}$, if $\Delta(r)$ (eq 21) is not zero, the Rabi frequency $\boldsymbol{\Omega}_{\mathrm{R}}$ is defined according to the work of Alvarellos et al. ${ }^{134}$ as

$$
\Omega_{\mathrm{R}}=\Omega_{\mathrm{R}}(r, t)=(2 \pi / h)\left\{\mathbf{W}(r, t)^{2}+\Delta(r)^{2}\right\}^{1 / 2}
$$

Here $\boldsymbol{\Omega}_{\mathrm{R}}$ coincides with the usual definition, given by eq 9 in the coordinate-independent representation for a resonant case, where $\Delta\left(r_{\text {res }}\right)=0$.

For randomly oriented molecular assemblies, we can replace $\exp ($ isr $)$ by $(1 / 4 \pi) \sin (\mathrm{sr}) /(\mathrm{sr})$ in eqs $23-25$. When the polarized laser light creates the ensemble with a spatial anisotropy, the molecular scattering intensities are not axially symmetric and clearly depend on the azimuthal angle $\varphi$. In this case, the scattering intensities for the ground and the excited states can be separated by a clear dependence on the azimuthal angle $\varphi$. This result is consistent with other publications. ${ }^{135,136}$ This population selection via the laser excitation polarization has been demonstrated but has not been fully exploited as yet. $^{137}$

The interference term, appearing in the molecular scattering of the electrons after the coherent excitation of the molecular system, provides the unique possibility to determine the offdiagonal elements of the density matrix and to conduct a tomographic reconstruction of the molecular quantum states in the limit of sufficiently short electron pulses. There is an additional requirement not included in this discussion in that the laser excitation pulse travels as the speed of light and the electron velocity is given by its kinetic energy. For $30 \mathrm{keV}$ electron energies, the electrons move at about $1 / 3$ the speed of light. The effective time resolution depends on the integrated signal as shown above and needs to take into account the sample geometry in which there is another integration over the sample path length. The difference in transit time of the laser excitation and electron pulse through the sample thickness is referred to as the velocity mismatch and will broaden the time resolution from the infinitely thin sample approximation used in the above equations. For solid state samples, the thickness to keep electron scattering in the single scattering limit treated above is on the order of $100 \mathrm{~nm}$ and there are negible velocity mismatch problems. However, for gas phase systems discussed above, the molecular beam or other effusive source has to be on the order of 100's of microns for sufficient signal, in which case temporal broadening due to velocity mismatch can be many picoseconds. This time average would completely wash out the signal and eliminate any chance of tomographic reconstruction. The introduction of relativistic electrons with the prospect of $10 \mathrm{fs}$ to even sub-femtosecond pulses of high brightness may open up this avenue, ${ }^{69}$ which will be an exciting development in imaging excited state molecular wave functions. In principle, this time resolution would allow imaging the time dependence of the initially prepared nuclear wavepackets and spreading along nonradiative channels, both reactive crossing and IVR processes. The interference term in eq 25 contains new information, unattainable by spectroscopic means, about the coherent nuclear dynamics of the molecular system that can, in principle, be obtained from the analysis of the detailed time series of the diffraction intensities observed on the relevant time scales.

The above treatment for an isolated 2-level molecular system was used to demonstrate the operating principles in the excitation and electron scattering probing the excited state dynamics. For molecular systems, there are multiple electronic states that need to be taken into account and the scaling to higher complexity necessarily introduces a large density of modes that lead to decoherence in the excited state preparation not treated above. For condensed phase systems, the coupling of the system to the bath leads to very fast electronic decoherence, as well as vibrational decoherence and relaxation processes. These decoherence processes localize the excited state wave function on the product state channel and are central to the chemical reaction dynamics. Without these processes, there would be no wave function collapse on a new electronic surface, and therefore no reaction. The above relations still hold with the caveat that the multiple electronic/vibronic states and decoherence/relaxation processes need to be included for a full description. For future development of the theory, it would also be desirable to include strong field excitation, which distorts the molecular potential (the so-called Keldysh mechanism; see, for example, ref 96, p. 214) and enable connection to strong field control of molecular dynamics.

\subsection{Cumulant Analysis of Gas Phase Electron Diffraction Data}

Following the phenomenological approach developed in ref 95, we describe the intensity function of diffracted electrons for an ensemble of molecular targets. This approach is in deference to a rigorous treatment of vibrational distributions and the details of the APES of the molecular system.

For randomly oriented molecules, the electron diffraction intensity is expressed in terms of cumulants (semi-invariants) of internuclear distances in a series that rapidly converges: ${ }^{95,126,136-138}$

$$
\begin{aligned}
s M(s, t)= & \sum_{i \neq j=1, N} g_{i j}(s) \operatorname{Im}\left\{\exp \left(i s r_{e, i j}\right) \sum_{k=0, \infty} \frac{i^{k}}{r_{e, i j}^{k+1}} \frac{\partial^{k}}{\partial s^{k}}\right. \\
& {\left.\left[\exp \left(\sum_{n=1, \infty} \frac{(i s)^{n}}{n !}\left\langle\Delta r_{i j}^{n}(t)\right\rangle_{c}\right)\right]\right\} }
\end{aligned}
$$

Here, $s$ is the magnitude of the scattering vector, $g_{i j}(s)$ are the scattering functions for atom pairs $i$ and $j$, and $\left\langle\Delta r_{\mathrm{ij}}^{\mathrm{n}}(t)\right\rangle_{\mathrm{c}}$ is the $n$ th-order cumulant, which is a nonlinear function of the moments of internuclear distance $r_{i j}(t) .{ }^{95}$ The first four cumulants in eq 27 have a clear meaning: they are the mean value, the dispersion, the skew, and the kurtosis of the corresponding probability density function, $P(r, t)$. Because no constraints are imposed regarding the molecular model, the cumulant expression introduces no unwarranted approximations in the analysis. Equation 25 can be reduced to the expression for the scattering signal conventionally used in 
traditional gas phase electron diffracton (GED) data refinements: ${ }^{131,139}$

$$
s M(s)=\sum_{i>j} \frac{g_{i j}(s)}{r_{a, i j}} \exp \left[-\frac{s^{2} l_{a, i j}^{2}}{2}\right] \sin \left[s r_{a, i j}-\kappa_{a, i j} s^{3}\right]
$$

The subscript $a$ in eq 28 denotes that averaging is performed with the internuclear probability density function $P_{T}(r) / r$, at temperature $T$. The $r_{a, i j}$ are the mean internuclear distances, and $l_{a, i j}$ is the root-mean-square amplitude of the nuclear motion. The parameter $\kappa_{a, i j}$ characterizes the asymmetry of the distribution function $P_{T}(r)$. Equation 28 describes the molecular intensity function for scattered electrons assuming an equilibrium ensemble of randomly oriented, quasi-rigid, identical molecules vibrating with small amplitude at a temperature $T$. Because of the so-called "shrinkage" of internuclear distances for vibrating molecules, the parameters $r_{a, i j}$ are possibly geometrically nonconsistent. ${ }^{131,139}$ For example, a symmetrical linear molecule $\mathrm{AB}_{2}$ appears bent with a temperature-dependent angle $(\mathrm{B}-\mathrm{A}-\mathrm{B})<180^{\circ}$. Therefore, information about the molecular symmetry and small distortions of the molecule from a high-symmetry configuration is difficult, or impossible, to obtain from conventional electron diffraction. But eq 27 makes it possible to derive the proper symmetry of the molecule as well as the Euclidian relations between internuclear distances in the framework of the molecular model of suggested symmetry. Moreover, it is possible to combine spectroscopic data and the results of quantum chemical calculations in the GED data refinement. $^{140}$

Quasi-Rigid Molecules. For small vibrational amplitudes, the APES of a quasi-rigid polyatomic molecule can be expanded in a series of natural, usually curvilinear, or linearized coordinates $q$ :

$$
U=\frac{1}{2} \sum_{i, j} f_{i j} q_{i} q_{j}+\frac{1}{6} \sum_{i j k} f_{i j k} q_{i} q_{j} q_{k}+\ldots
$$

It can also be expressed in a basis of normal coordinates $Q$ :

$$
U=\frac{1}{2} \sum_{k} \omega_{k}^{2} Q_{k}^{2}+\frac{1}{6} \sum_{k l m} \delta_{k l m} Q_{k} Q_{l} Q_{m}+\ldots
$$

Here, $f_{i j}$ are quadratic and $f_{i j k}$ are cubic force constants, $\omega_{k}$ are the frequencies of the harmonic normal modes, and $\delta_{i j k}$ are the cubic force constants expressed in normal coordinates. The cumulants of eq 27 are connected by the well-known relationships with the moments of internuclear distances, which in turn are expressed through the moments of internal vibrational coordinates. It is easy to calculate the thermally averaged powers of the normal coordinates. The expressions for the cumulants in terms of the average values of the products of the normal coordinates are obtained by expanding each internuclear distance into a series with respect to the normal mode coordinates $Q$ and carrying out the averaging over the vibrational states (see monograph by Ischenko et al. ${ }^{95}$ ).

For harmonic vibrations, only the averages of the even powers of the normal coordinates differ from zero:

$$
\begin{gathered}
\left\langle Q_{k}^{2}\right\rangle=\frac{\hbar}{2 \omega_{k}} \operatorname{cth} \frac{\hbar \omega_{k}}{2 k T} ; \quad\left\langle Q_{k}^{4}\right\rangle=3\left\langle Q_{k}^{2}\right\rangle^{2} ; \\
\left\langle Q_{k}^{2} Q_{l}^{2}\right\rangle=\left\langle Q_{k}^{2}\right\rangle\left\langle Q_{l}{ }^{2}\right\rangle \text { when } k \neq l
\end{gathered}
$$

For anharmonic functions of the potential energy (eq 30), first-order perturbation theory shows that the mean values of the even powers of the coordinates do not change. The leading linear correction of the distances is determined by the mean values of the normal coordinates:

$$
\left\langle Q_{k}\right\rangle=-\frac{1}{2 \omega_{k}^{2}} \sum_{l} \delta_{k l}\left\langle Q_{l}^{2}\right\rangle
$$

The third orders of the normal coordinates are required to calculate the asymmetry of the distribution of the distances (i.e., the third-order cumulants). Considering the previously mentioned methods of calculating average thermodynamic quantities, the average values of the third powers of the normal coordinates are given by

$$
\begin{aligned}
\left\langle Q_{k}^{3}\right\rangle= & 3\left\langle Q_{k}\right\rangle\left\langle Q_{k}^{2}\right\rangle+\delta_{k k k} X_{k k k} ; \\
\left\langle Q_{k}^{2} Q_{l}\right\rangle=\left\langle Q_{l}\right\rangle\left\langle Q_{k}^{2}\right\rangle+\delta_{k k l} X_{k k l} \quad \text { when } k \neq l ; & \text { when } k \neq l, k \neq m, m \neq l \\
\left\langle Q_{k} Q_{l} Q_{m}\right\rangle=\delta_{k l m} X_{k l m} \quad & \\
X_{k l m}= & -\frac{\hbar^{3}}{32 k T \omega_{k} \omega_{l} \omega_{m} \operatorname{sh}\left(z_{k}\right) \operatorname{sh}\left(z_{l}\right) \operatorname{sh}\left(z_{m}\right)} \\
& \times\left\{\frac{\operatorname{sh}\left(z_{k}+z_{l}+z_{m}\right)}{z_{k}+z_{l}+z_{m}}+\frac{\operatorname{sh}\left(z_{k}-z_{l}+z_{m}\right)}{z_{k}-z_{l}+z_{m}}\right. \\
& \left.+\frac{\operatorname{sh}\left(z_{k}+z_{l}-z_{l}\right)}{z_{k}+z_{l}-z_{m}}+\frac{\operatorname{sh}\left(z_{k}-z_{l}-z_{m}\right)}{z_{k}-z_{l}-z_{m}}\right\}
\end{aligned}
$$

Here, the abbreviation $z=h \omega / 4 k T$ is used. This result involves averaging the values of the individual vibrational levels calculated using first-order perturbation theory. We note that the equation presented in ref 141 can be reduced to the same form and that a more general theory, based on the Bloch equation, leads to the same result. ${ }^{142,143}$ Thus, the thermally average distances $r_{a}$ for eq 28 or $r_{g}=r_{a}+l_{a}^{2} / r_{a}$ with $l_{a}$ the mean vibrational amplitude can be calculated from the equilibrium parameters (and vice versa), provided the quadratic and cubic terms of the expansion for the APES of the molecule are known. ${ }^{95}$ Even so, the thermally averaged parameters are often determined from experimental data without consideration of the asymmetry of the probability density distribution $P_{T}(r)$.

Analysis of Intramolecular Motions. Within the BornOppenheimer approximation, equations for molecular models can be derived for small amplitudes of the nonvibronic vibrational modes. ${ }^{144-146}$ Braun and Kiselev have given the complete formulation of this derivation on the basis of adiabatic perturbation theory. ${ }^{147}$ For molecules undergoing chemical reactions where at least one degree of freedom involves large amplitude motion, this approach needs to be modified by explicitly separating large and small amplitude coordinates. This was done in ref 140 and later applied to polyatomic molecules. ${ }^{148-150}$ The standard method, which for small displacements converges rapidly, describes the displacement of the center of mass of the molecule and the rotation of the molecule as an integral unit. The internal coordinates are determined as the displacement of the nuclei in relation to the equilibrium configuration: $\left\{\mathbf{R}^{0}\right\}=\left\{\mathbf{R}_{1}^{0}, \ldots, \mathbf{R}_{N}^{0}\right\}$.

For large amplitude motions, it is necessary to introduce generalized coordinates that explicitly separate the coordinates connected with the large amplitude motion. This is done by examining the pseudoequilibrium configurations (quasi-con- 
formers) such that the small vibrations can be expressed relative to these configurations. Suppose that $m$ coordinates describe the large amplitude motion $\rho_{i}(i=1, \ldots, m)$. For each set of these coordinates, the pseudoequilibrium configuration $\left\{\mathbf{R}^{0}(\rho)\right\}$, corresponding to the minimum of the APES with respect to all remaining degrees of freedom of the molecule, is found. The remaining internal coordinates $q_{k}(k=1, \ldots, n)$ are then determined as displacements relative to the pseudoequilibrium configurations.

Construction of the Hamiltonian. The first step is the transition from Cartesian coordinates to a new system of coordinates, which consists of the displacement of the molecular center $\mathbf{R}$, the Euler angles $\theta$ that describe the molecular orientation, and the internal coordinates of the nuclei $\{\rho, q\}$ :

$$
\mathbf{R}_{i}^{\prime}=\mathbf{R}+C(\theta)\left[\mathbf{R}_{i}^{0}(\rho)+\sum_{k=1}^{n} \mathbf{A}_{i k}(\rho) q_{k}\right]
$$

where $C(\theta)$ is the rotation matrix. ${ }^{151}$ One obtains the Hamiltonian as

$$
\begin{aligned}
\hat{H}= & \frac{1}{2 m} \sum_{k} \hat{\mathbf{P}}_{k}^{2}+\frac{1}{2 M}\left(\hat{\mathbf{P}}-\hat{\mathbf{P}}_{e}\right)^{2}+\frac{1}{2} \hat{P}^{*} G \hat{P}+\frac{1}{2}(\hat{\pi}-\hat{b})^{*} S(\hat{\pi}-\hat{b}) \\
& +\frac{1}{2}\left(\hat{\mathbf{M}}-\hat{\mathbf{M}}_{e}-\hat{\mathbf{M}}_{\rho}-\hat{\mathbf{M}}_{v}\right)^{*} \sigma\left(\hat{\mathbf{M}}-\hat{\mathbf{M}}_{e}-\hat{\mathbf{M}}_{\rho}-\hat{\mathbf{M}}_{v}\right)+U \\
& +F(\rho, q)
\end{aligned}
$$

In eq $35, M$ is the mass of the molecule, $m$ is the electron mass, $\hat{\mathbf{P}}$ and $\hat{\mathbf{M}}$ are the momentum and angular momentum operators of the entire molecule, and $\hat{p}$ and $\hat{\mathbf{M}}_{v}$ are operators of momentum and angular momentum for the small amplitude motions. $\hat{\pi}$ and $\hat{\mathbf{M}}_{\rho}$ are operators for the momentum and angular momentum of the large amplitude motion displacement, and $\hat{b}$ describes the interaction between these motions. $G, S$, and $\sigma$ are matrices that depend on the coordinates. Detailed expressions for all these have been given in refs 95 and 149.

For small displacements along the internal coordinates $q$, the terms in eq 35 can be arranged by powers of these coordinates, leading to the representation:

$$
\begin{aligned}
& \hat{H}=\hat{H}^{(0)}+\hat{H}^{(1)}+\hat{H}^{(2)}+\ldots \\
& \hat{H}^{(i)}=\hat{T}^{(i)}+U^{(i)}
\end{aligned}
$$

Adiabatic Potential Energy Surface: Small Amplitude Vibrations. We examine several partial problems whose solutions can be used to construct approximations for the eigenvalues and the Hamiltonian operator (eq 35). We assume that all nuclei are at rest in a configuration described by the coordinates $(\rho, q)$ :

$$
\hat{H}_{e l} \psi_{e} \equiv\left(\hat{H}^{(0)}+U\right) \psi_{e}=V \psi_{e}
$$

The eigenvalues and functions depend parametrically on the nuclear coordinates. The energy levels $V_{n}(\rho, q)$, where the index $n$ denotes the electronic states, are the APES. In principle, the functions $V_{n}$ for the ground and excited electronic states can be directly obtained from quantum mechanics calculations.

The solution of eq 37 is written as a series with respect to the degrees of the coordinates $q$ :

$$
\begin{aligned}
& \psi_{\mathrm{en}}=\psi_{\mathrm{en}}^{(0)}(\rho, \mathbf{r})+\psi_{\mathrm{en}}^{(1)}(\rho, q, \mathbf{r})+\psi_{\mathrm{en}}^{(2)}(\rho, q, \mathbf{r})+\ldots \\
& V_{n}=V_{n}^{(0)}(\rho)+V_{n}^{(1)}(\rho, q)+V_{n}^{(2)}(\rho, q)+\ldots
\end{aligned}
$$

We note that $V^{(1)}$ disappears if the pseudoequilibrium configurations $\left\{\mathbf{R}^{(0)}(\rho)\right\}$ can be represented by the configurations in which the energy minimum is reached in the coordinates $q$ (at fixed values of the coordinates $\rho$ ).

Next we take into account the small vibrations of the nuclei while keeping the coordinates of large amplitude "frozen". We examine the Hamiltonian:

$$
\hat{H}^{\prime}=\hat{T}^{(0)}+\hat{T}^{(2)}+U=\hat{H}_{\mathrm{el}}+\hat{T}^{(2)}
$$

The coordinate $\rho$ is included in this operator as a parameter, so that the eigenvalue problem, $\hat{H}^{\prime} \psi^{\prime}=E \psi^{\prime}$, has eigenfunctions and values that depend on $\rho: \psi^{\prime}=\psi^{\prime}(\rho, q, r), E=E(\rho)$. These functions can again be expanded with respect to the eigenfunctions of the previous problem

$$
\psi^{\prime}(\rho, q, r)=\sum_{k} a_{k}(\rho, q) \psi_{e k}(\rho, q, \mathbf{r})
$$

With this substitution one obtains

$$
\sum_{k}\left(\hat{T}^{(2)} a_{k}\right) \psi_{\mathrm{ek}}+\sum_{k}\left(\hat{T}^{(2)} \psi_{\mathrm{ek}}\right) a_{k}=\sum_{k} a_{k}\left(E-V_{k}\right) \psi_{\mathrm{ek}}
$$

For the $n$th electronic state and for small amplitude vibrations one obtains:

$$
\left(\hat{T}^{(2)}+V_{n}\right) a_{n}+\sum_{k} F_{k n} a_{k}=E a_{n}
$$

where

$$
F_{k n}(\rho, q)=\int \psi_{e k}^{*}\left(\hat{T}^{(2)} \psi_{e n}\right) \mathrm{d} \mathbf{r}
$$

is responsible for the vibronic effect. Expanding all the terms of eq 42 into a series with respect to the degrees of the coordinates $q$, one finds for the state $(0, v)$ :

$$
\begin{aligned}
E_{0 v}(\rho)= & V_{0}^{(0)}(\rho)+\varepsilon_{0 v}(\rho)+\left[V_{0}^{(4)}+F_{00}^{(4)}\right]_{v v} \\
& +\sum_{v^{\prime} \neq v} \frac{\left[V_{0}^{(3)}\right]_{v v^{\prime}}^{2}}{\varepsilon_{0 v}-\varepsilon_{0 v^{\prime}}}+\ldots \\
\psi_{0 v}^{\prime}(\rho, q, \mathbf{r})= & \left\{f_{0 v}(\rho, q)\right. \\
& \left.+\sum_{v^{\prime} \neq v} \frac{\left[V_{0}^{(3)}\right]_{v v^{\prime}}}{\varepsilon_{0 v}-\varepsilon_{0 v^{\prime}}} f_{0 v^{\prime}}(\rho, q)+\ldots\right\} \psi_{e 0}(\rho, q, \mathbf{r})
\end{aligned}
$$

Large Amplitude Motion. For large amplitude motions, the solution of the Hamiltonian (eq 35) is written as a series with respect to the solutions of the previous task: ${ }^{95,151}$

$$
\psi(\mathbf{R}, \theta, \rho, q, \mathbf{r})=\sum_{n v^{\prime}} R_{n v^{\prime}}(\mathbf{R}, \theta, \rho) \psi_{n v^{\prime}}^{\prime}(\rho, q, \mathbf{r})
$$

Averaging over eigenvalues for the electronic coordinates and over the coordinates of the small amplitude vibrations yields 


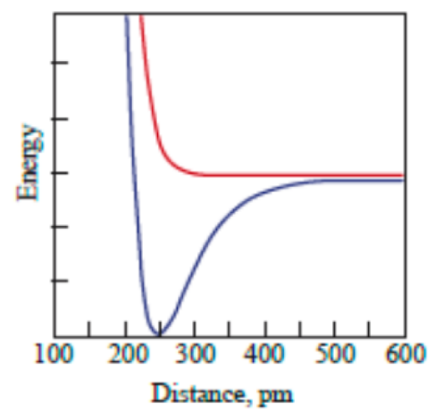

$$
\begin{aligned}
& \operatorname{IBr}\left(\mathrm{X}^{1} \Sigma_{0}^{+}\right) \rightarrow \operatorname{IBr}\left(\mathrm{B}^{3} \Pi_{0}^{+}\right), 400<\lambda<620 \mathrm{~nm} \\
& \operatorname{IBr}\left(\mathrm{B}^{3} \Pi_{0}^{+}\right) \rightarrow \operatorname{I}+\operatorname{Br}\left({ }^{3} \mathrm{P}_{1 / 2}\right), \lambda<256 \mathrm{~nm} \\
& \operatorname{IBr}\left(\mathrm{B}^{3} \Pi_{0}^{+}\right) \rightarrow \operatorname{IBr}\left(\mathrm{BO}^{+}\right) \rightarrow \mathrm{I}+\operatorname{Br}\left({ }^{2} \mathrm{P}_{3 / 2}\right)
\end{aligned}
$$

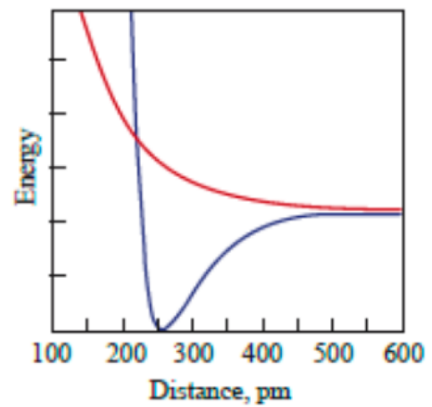

$$
\begin{aligned}
& \mathrm{ICN} \rightarrow \mathrm{I}\left({ }^{2} \mathrm{P}_{3 / 2}\right)+\mathrm{CN}\left(\mathrm{X}^{2} \Sigma^{+}\right) \\
& \mathrm{ICN} \rightarrow \mathrm{I}\left({ }^{2} \mathrm{P}_{1 / 4}\right)+\mathrm{CN}\left(\mathrm{X}^{2} \Sigma^{+}\right)
\end{aligned}
$$

$\operatorname{IBr} 25 \mathrm{fs}$ probe $(\sigma=0,15)$

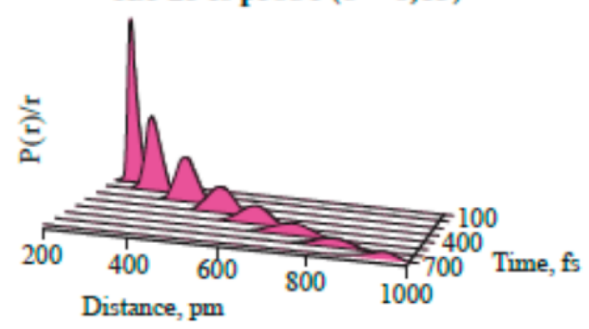

a)

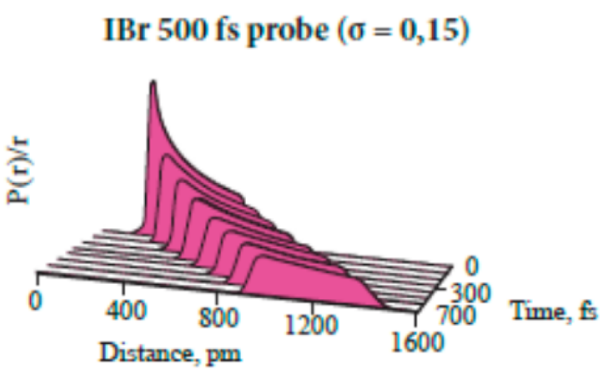

b)

Figure 8. (a) The radial distribution curves for IBr molecules in the Gaussian approximation of the wave packets, averaged over $25 \mathrm{fs}$ and displayed every $100 \mathrm{fs}$. Here $\sigma$ is the parameter accounting for the spreading of the wave packet. (b) The radial distribution curves for IBr in the Gaussian approximation of the wave packets averaged over $500 \mathrm{fs}$. It can be seen that the longer electron pulses do not allow one to resolve the actual profile of the wave packet, but some important details are still visible. The upper part of the figure shows the potential energy functions for the ground and the excited states of the molecules IBr and ICN. Reproduced with permission from ref 127. Copyright 1997 Oxford University Press.

$$
\sum_{n v^{\prime}}\left(\int \psi_{o v}^{\prime *}\left[\hat{T}^{(4)}+\hat{T}^{(5)}+\ldots\right] R_{n v^{\prime}} \psi_{n v^{\prime}}^{\prime} d q d \mathbf{r}\right)+E_{0 v}(\rho) R_{0 v}=E R_{0 v}
$$

To first approximation, only the term $\mathrm{R}_{0 v}^{(0)}(\mathbf{R}, \boldsymbol{\theta}, \rho)$ differs from zero:

$$
\left[\hat{T}_{00, v v}^{(4)}(\rho)+E_{0 v}(\rho)\right] R_{0 v}^{(0)}=E R_{0 v}^{(0)}
$$

This is the general equation for molecular systems with large amplitude motions. Two important practical cases can be considered.

Nonlinear Molecules. Provided that the quanta of small amplitude motions are considerably larger than rotational quanta, one can separate the large amplitude motion from the rotational effects. One finds the equation with the kinetic energy operator

$$
\begin{aligned}
\hat{T}_{v v}^{(4 a)} & =\frac{1}{2}\left[(\hat{\pi}-\hat{b}) * S^{(0)}(\hat{\pi}-\hat{b})\right]_{v v}+F^{(0)}(\rho) \\
& =\frac{1}{2} \hat{\pi}^{*} S^{(0)} \hat{\pi}+F^{(0)}(\rho)+F_{v v}^{(4)}(\rho)
\end{aligned}
$$

Linear Molecules. Here it may not be possible to separate the rotation from large amplitude vibrations. This is the case, for example, for the almost free internal rotation in a molecule with energies on the order of $1 \mathrm{~cm}^{-1}$. For a linear molecule, we have

$$
\hat{T}_{v v}^{(4 a)}=\frac{1}{2} \hat{\pi}^{*} S^{(0)} \hat{\pi}+\frac{1}{2} \hat{M}_{z} \sigma_{z z}^{(0)} M_{z}+F^{(0)}(\rho)+F_{v v}^{(4)}(\rho)
$$

Additional effects, such as the rotation in other directions, can be treated using higher levels of perturbation theory. For a linear molecule with only one large amplitude motion, substitution of the operator (eq 50) into the Schrödinger equation gives

$$
-\frac{\hbar^{2}}{2} \frac{\partial}{\partial \rho} S^{(0)}(\rho) \frac{\partial \psi}{\partial \rho}-\frac{\hbar^{2}}{2} \sigma_{z z}^{(0)}(\rho) \frac{\partial^{2} \psi}{\partial \phi^{2}}+V_{\text {eff }}(\rho) \psi=E \psi
$$

with an effective potential described by

$$
V_{\text {eff }}(\rho)=V_{0}^{(0)}(\rho)+F^{(0)}(\rho)+\sum_{k=1}^{n} \hbar \omega_{k}(\rho)\left(v_{k}+1 / 2\right)+F_{v v}(\rho)
$$

which includes only the large amplitude coordinates. 
that depends on the vibrational state $v=\left\{v_{1}, \ldots, v_{n}\right\}$. Here, $\omega_{\mathrm{k}}(\rho)$ corresponds to the frequencies of the small amplitude vibrations, and $F_{v v}(\rho)$ is found by averaging the interactions ( $q$ $-\rho$ ) over the $v$ th vibrational state. In most cases, it is sufficient to consider the effective potential for the ground state. The Watson function $F(\rho)$ above has the form: ${ }^{95}$

$$
F(\rho)=\frac{\hbar^{2}}{8} S^{(0)}\left[\frac{\partial^{2} \ln \gamma}{\partial \rho^{2}}+\frac{1}{4}\left(\frac{\partial \ln \gamma}{\partial \rho}\right)^{2}\right]+\frac{\hbar^{2}}{8} \frac{\partial S^{(0)}}{\partial \rho} \frac{\partial \ln \gamma}{\partial \rho}
$$

where

$$
\gamma(\rho)=\frac{1}{S^{(0)}(\rho) \sigma_{z z}^{(0)}(\rho)}
$$

For a linear molecule, this can be reduced to a onedimensional equation if the eigenfunctions are taken to be in the form $\psi(\rho, \varphi)=R(\rho) \exp (i l \varphi)$, where $l$ is the angular quantum number. In this case, one has

$$
-\frac{\hbar^{2}}{2} \frac{\partial}{\partial \rho} S^{(0)}(\rho) \frac{\partial R}{\partial \rho}+\left[V_{\text {eff }}(\rho)+\frac{\hbar^{2} l^{2}}{2} \sigma_{z z}^{(0)}(\rho)\right] R=E R
$$

which can be solved numerically.

On the basis of this discussion, the total eigenfunction of the molecular system is represented as a product of functions that correspond to different types of motions. The equations for small amplitude vibrations are solved as harmonic oscillators with anharmonicity corrections as needed. Numerical methods are used to solve the equations for the large amplitude motions.

\subsection{Dynamics of Dissociation}

To illustrate the basic effects arising from time-dependent molecular structures in the scattering intensities and their corresponding Fourier transforms (the radial distribution functions of the interatomic distances), we will focus on diatomic $(\mathrm{A}-\mathrm{B})$ and linear triatomic $(\mathrm{A}-\mathrm{B}-\mathrm{C})$ molecules in which the action of a laser pulse breaks the bond $A-B$. In many cases, the potential function for such systems can be expressed as $^{152}$

$$
V(R, r)=V_{0} \exp [-(R-\gamma r) / \rho]
$$

where $R$ is the distance between the nuclei $A$ and $B$ in the molecule $\mathrm{A}-\mathrm{B}$, or the distance between the nucleus $\mathrm{A}$ and the center of mass of the fragment $\mathrm{B}-\mathrm{C}$ in the molecule $\mathrm{A}-\mathrm{B}-\mathrm{C}$. Further, $\gamma=m_{\mathrm{B}} \mathrm{m}_{\mathrm{C}} /\left(m_{\mathrm{B}}+m_{\mathrm{C}}\right)$ is the reduced mass and $r=$ $r_{0}(\mathrm{BC})-r_{\mathrm{e}}(\mathrm{BC})$ with $r_{0}(\mathrm{BC})$ and $r_{\mathrm{e}}(\mathrm{BC})$ the internuclear distances in the ground vibrational state and the equilibrium internuclear distance of the fragment B-C, respectively. Finally, $\rho$ is a so-called range parameter. ${ }^{152,153}$ The reactions and PES of this kind are well-known for a number of molecular systems. ${ }^{152-154}$ In the first stage of the analysis, the manifestation of the nuclear dynamics in the scattering of ultrashort electron pulses has been explored for adiabatic potential functions.

Using $\mathrm{IBr}$ as an example, the photoexcitation and subsequent dissociation can be divided into 3 possible photoprocesses as shown in Figure 8. The photoexcitation is accompanied either by the dissociation of the diabatic type, yielding $\mathrm{Br}$ atoms in the excited state, or by the dissociation of adiabatic type, in which the molecule dissociates into atoms in ground states. Figure 8 assumes a $\delta$-function for the excitation pulse and the electron pulse as a Gaussian function with a duration of $\tau$ as defined above. The time-dependent intensities of the molecular scattering and the corresponding radial distributions of the internuclear distances during the photodissociation of $\mathrm{IBr}$ and ICN have been calculated (see Figures 8 and 9). ${ }^{127}$ From
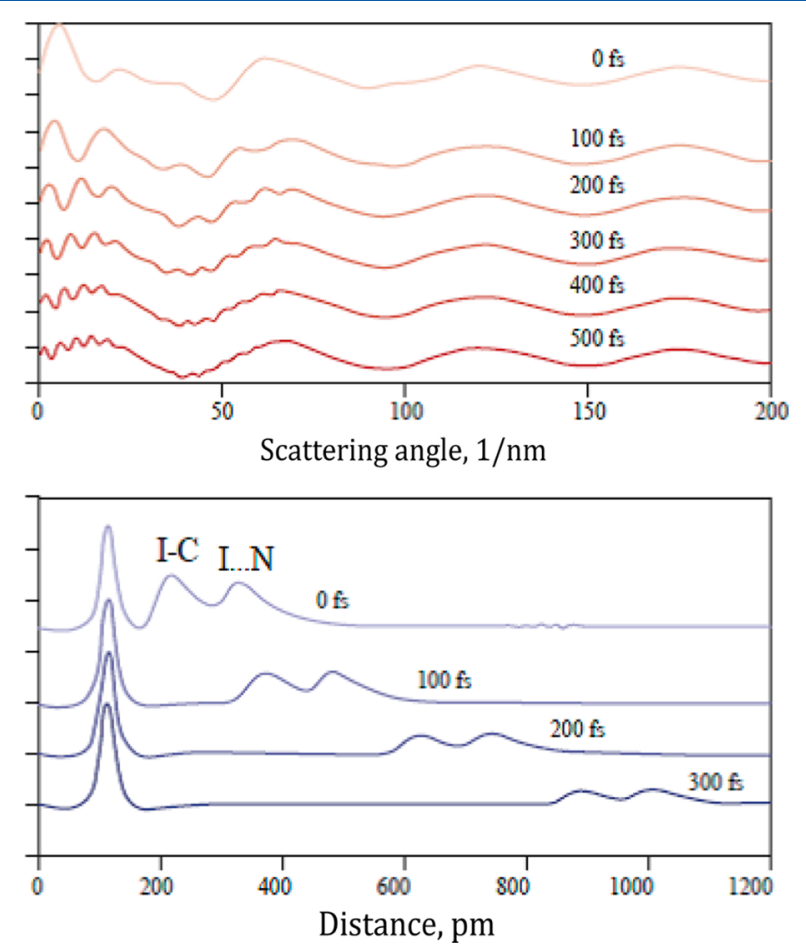

Figure 9. Manifestation of the structural dynamics of the dissociation of I-CN for time-dependent molecular scattering intensities (the upper group of the curves) and for the radial distributions (the lower curves). Reproduced with permission from ref 127. Copyright 1997 Oxford University Press.

Figure $8 \mathrm{~b}$, it is clear that the radial distribution curves obtained with relatively long probe pulses are significantly different from those presented in Figure 8a. However, some details of the dynamics of the wave packets still are revealed at a time interval that is shorter than the probe pulse duration. Figure 8 also shows that the distribution of the time-dependent wave packet can be observed using the time-resolved electron diffraction technique, if the probing electron pulse is short enough.

These studies show that the time-dependent intensity of the electron diffraction observed for dissociative states is strongly dependent on the shape of the PES. The investigations also demonstrate that the diffraction intensities in TRED can be easily calculated for dissociative processes, provided that the potential energy is known. Thus, in principle it is possible to solve the inversion problem. This statement only holds true if the PES of the excited state is known accurately enough and then the diffraction phases can be assigned. The experimental observations can then be used to refine the PES as normally employed for the use of theory in structural refinement. Generally, the PES is not known very accurately, especially for reactive surfaces, and one is dealing with many more diffraction orders than for simple 2 and 3 atom systems for which the phase ambiguity can not readily be resolved without going to very high level theory to better approximate the PES. It is here where new methods are needed to provide solutions to the inversion problem (vide infra). It is noteworthy that these calculations show the needed time resolution of approximately 
$100 \mathrm{fs}$ for dissociative channels as discussed above. Close inspection shows that this class of experiments gives not only dynamical information but also information on the nuclear probability distribution of the excited wavepacket. This information goes well beyond other forms of spectroscopy. Recent experiments have further amplified this point in the study of the excited state vibrational wavepackets of $\mathrm{I}_{2}$. ${ }^{155}$

\subsection{Solution of the Inversion Problem for Gas Phase} Systems

The averaging of the molecular scattering intensity $\operatorname{sM}(s, t)$ function over the temporal profile of the electron bunch $I_{0}(t$; $\left.t_{d}\right)$ gives the intensity of the diffraction in TRED, $s M\left(s ; t_{d}\right)$, with parametric dependence on the delay time $t_{d}$ between the laser pump pulse and the pulse of the electron probe $\tau$ is

$$
\left\langle s M\left(s ; t_{\mathrm{d}}\right)\right\rangle_{\tau}=\int_{\mathrm{t}, \mathrm{t}+\tau} I_{0}\left(t^{\prime} ; t_{\mathrm{d}}\right) s M\left(s, t^{\prime}\right) \mathrm{dt}^{\prime}
$$

In this case, to solve the inversion problem, or to revise the parameters in the model used, it is necessary to minimize the functional

$$
\sum_{\mathrm{i}=1, \mathrm{~m}}\left[\left\langle s_{\mathrm{i}} M\left(s_{\mathrm{i}} ; t_{\mathrm{d}}\right)\right\rangle_{\tau, \exp }-R\left\langle s_{\mathrm{i}} M\left(s_{\mathrm{i}} ; t_{\mathrm{d}}\right)\right\rangle_{\tau, \text { theo }}\right]^{2}
$$

where $m$ is the number of points of the experimental values of the scattering intensity $s_{i} M\left(s_{i} ; t_{\mathrm{d}}\right)$ for the temporal delay $t_{\mathrm{d}}$ and $R$ is the index of the resolution. The solution of the inversion problem in diffraction is an ill-posed problem. To solve the multiparameter problems, it is possible to use the rather effective method of regularization. ${ }^{156-158}$ In this case, it is possible to extract a so-called quasi-solution by minimizing the functional

$$
\begin{aligned}
\mathbf{M}^{\alpha}= & \sum_{\mathrm{i}=1, \mathrm{~m}}\left[\left\langle s_{\mathrm{i}} M\left(s_{\mathrm{i}} ; t_{\mathrm{d}}\right)\right\rangle_{\tau, \exp }-R\left\langle s_{\mathrm{i}} M\left(s_{\mathrm{i}} ; t_{\mathrm{d}}\right)\right\rangle_{\tau, \text { theo }}\right]^{2} \\
& +\alpha \Omega\left[\left\langle P\left(r ; t_{\mathrm{d}}\right)\right\rangle_{\tau}\right] \rightarrow \min
\end{aligned}
$$

where the stabilizing term $\Omega\left(P\left(r ; t_{\mathrm{d}}\right)\right)_{\tau}$ is expressed by a polynomial

$$
\Omega\left[\left\langle P\left(r ; t_{\mathrm{d}}\right)\right\rangle_{\tau}\right]=\sum \sum_{i \neq j=1, N} \int \sum_{n=0, p} q_{n}\left(r_{i j}\right)\left[d^{n}\left\langle P\left(r ; t_{\mathrm{d}}\right)\right\rangle_{\tau} / \mathrm{dr}_{\mathrm{ij}}{ }^{n}\right]^{2} \mathrm{dr}_{\mathrm{ij}}
$$

where $q_{n}\left(r_{i j}\right)$ are the non-negative functions and $\left\langle P\left(r ; t_{\mathrm{d}}\right)\right\rangle_{\tau}$ is the probability density of the distribution of interatomic distances for the temporal delay $t_{\mathrm{d}}$ averaged over time $t$.

Two approaches to the choice of the regularization parameter $\alpha$ have been tested. In the first technique, the parameter $\alpha$ has been calculated in accordance with the study of $^{159,160}$

$$
\begin{aligned}
& \alpha=\alpha(\sigma, E)=\sigma^{2} / E^{2} \\
& \sigma^{2} \geq\left\|\left\langle s_{\mathrm{i}} M\left(s_{\mathrm{i}} ; t_{\mathrm{d}}\right)\right\rangle_{\tau, \exp }-R\left\langle s_{\mathrm{i}} M\left(s_{\mathrm{i}} ; t_{\mathrm{d}}\right)\right\rangle_{\tau, \text { theo }}\right\| \\
& E^{2} \geq \sum \sum_{\mathrm{i} \neq \mathrm{j}=1, N} \int \sum_{n=0, p} q_{n}\left(r_{\mathrm{ij}}\right)\left[d^{n}\left\langle P\left(r ; t_{\mathrm{d}}\right)\right\rangle_{\tau} / \mathrm{d} r_{\mathrm{ij}}{ }^{\mathrm{n}}\right]^{2} \mathrm{~d} r_{\mathrm{ij}}
\end{aligned}
$$

The method of regularization described in the papers by Miller and Viano ${ }^{159,160}$ was chosen because it allows us to estimate the accuracy of parameters. In an alternative approach by Bertero et al., ${ }^{161}$ the regularization parameter $\alpha$ can be optimized by minimizing the functional

$$
\begin{aligned}
\mathbf{R}_{\mathrm{f}}^{2}\left(\alpha, \xi^{\alpha}\right)= & \sum_{\mathrm{i}=1, \mathrm{~m}}\left[\left\langle s_{i} M\left(s_{i} ; t_{\mathrm{d}}\right)\right\rangle_{\tau, \exp }-R\left\langle s_{i} M\left(s_{i} ;_{t_{\mathrm{d}}}, \xi^{\alpha}\right)\right\rangle_{\tau, \tau \text { heo }}\right]^{2} / \\
& \sum_{\mathrm{i}=1, \mathrm{~m}}\left[\left\langle s_{i} M\left(s_{i} ; t_{\mathrm{d}}\right)\right\rangle_{\tau, \exp }\right]^{2}
\end{aligned}
$$

where $\xi^{\alpha}$ is the set of refined parameters that depending on the regularization parameter $\alpha$ as described in the work of Ho and Rabitz. ${ }^{162,163}$ The use of the regularization method in TREDexperiments is described in ref 133. It is shown that the stabilizing functional $\Omega\left(P\left(r ; t_{\mathrm{d}}\right)\right)$ makes it possible to significantly stabilize the minimization process. ${ }^{126}$ This approach can not be strictly applied to solid state systems as the phases defined by the crystalline lattice add to the uncertainty and new methods of introducing constraints are needed as will be discussed below.

It should be mentioned that a very similar approach has been recently used by Minitti et al. ${ }^{164}$ to help reconstruct the ringopening reaction of cyclohexadience using femtosecond X-ray pulses from an X-ray free electron laser (XFEL). In the X-ray case, it is remarkable that sufficient diffraction was observable given the extremely small scattering cross section, and this again reinforces the importance in source brightness. The X-ray wavelength was $1.5 \AA$ compared to less than $0.1 \AA$ for electron sources, and reasonable detector conditions resulted in only two diffraction orders. Nevertheless, by using theoretical calculations for many trajectories, it was possible to achieve a fit to give the time-dependent dynamics in the spirit of the above formulation.

\subsection{Difference Method for Time-Dependent Diffraction Data Analysis}

The above treatment has assumed $100 \%$ excitation. This is not the case for most experimental conditions, especially using femtosecond laser excitation where it is imperative to keep the peak intensity of the excitation pulse low to avoid multiphoton ionization artifacts. Any residual background will lead to noise and signal unrelated to the dynamics of interest. It is standard practice in femtosecond spectroscopy to use differential detection to remove this background. Even so, there remains noise from the unexcited background relative to the small signal of interest. For all-optical methods, the ability to extract small signals from this background depends on the sampling rate (pump-probe repetition) and the laser/detector noise within some effective measuring bandwidth. It is possible to measure changes as small as $10^{-8}$ within the detector noise floor, with $10^{-4}$ more typical for most experimental conditions. ${ }^{165}$ However, for diffraction experiments, there are many signal components stemming from the fact that one attempts to reconstruct an image, rather than an integrated intensity modulation of the probe at a single point of observation.

Typically there is a trade-off in the degree of excitation and the need to observe sufficient changes in intensity in the different orders to reconstruct the time-dependent structures. As a rule of thumb, for picosecond and subpicosecond experiments, excitation levels of no more than approximately $10 \%$ are required in order to stay within the linear response and avoid multiphoton ionization. This is only a guideline as there are trade-offs in excitation peak power, number of shots averaged, laser noise, electron source noise, and detector noise. Within this limit, the recorded diffraction pattern intensities are linearly dependent on the excitation intensity and the observed dynamics corresponding to photochemical or photophysical processes from a well-defined initially prepared state, as opposed to a distribution of multiphoton excited states and 
multiphoton ionization. Denoting $\Delta I\left(s ; t_{\mathrm{ref}}, t\right)$ as the difference curves related to the structural changes ensuing from the initially prepared state, we get the differential signal:

$$
\Delta I\left(s ; t_{\text {ref }}, t\right)=I\left(s ; t_{\text {ref }}\right)-I(s ; t)
$$

to yield

$$
\Delta s M\left(s ; t_{\text {ref }}, t\right)=s M\left(s ; t_{\text {ref }}\right)-s M(s ; t)
$$

The difference radial distribution curves $\Delta f\left(r ; t_{\text {ref }} t\right)$ in the space of interatomic distances $r$ is then obtained by Fourier transforming the difference curves $\Delta s M\left(s ; t_{\text {ref }}, t\right)$.

The calculation of the theoretical equivalent of the timedependent scattering intensity with the nonequilibrium distribution in this system can be performed using the methods discussed above, where the excitation fraction is needed to properly weight the changes in diffraction intensities of the different orders as shown by Coppens et al. for the X-ray case. $^{166}$

\subsection{Time-Dependent Diffraction from Single Crystals:}

\section{Solution to the Inversion Problem}

The highest spatial resolution of structures is obtained from single crystals. This fact is owed to the amplification factor of $\mathrm{N}^{\prime 2}$ in diffraction and the further amplification into specific diffraction orders reflecting the underlying lattice periodicity and alignment of all the molecules into the particular symmetry group of the crystalline lattice. Electrons scatter off the electrostatic potential, whereas X-rays scatter from the phase shift introduced by the induced polarization related to the electron density. The difference in scattering mechanisms, apart from the more than 10 times shorter wavelength typically for electrons over X-rays, makes electron diffraction more sensitive to nuclear positions and with this higher spatial resolution. For certain chemical reactions, spatial resolution on the order of 0.01-0.1 A (vide supra) is needed as even such small motions significantly contribute to the reaction energetics. Such spatial resolution is further needed to rigously compare to theory and help refine theoretical methods. Electron diffraction is wellcapable of this level of spatial resolution for single crystal studies. As above with gas phase diffraction, the inversion from reciprocal space to real space is challenging.

The electrostatic potential giving rise to the observable is defined by ${ }^{167}$

$$
\phi(\mathbf{k})=\frac{1}{\Omega} \sum_{j=1}^{N} \frac{f_{j}^{B}(\mathbf{k})}{A} e^{\mathrm{ik} \cdot \mathbf{r}_{j}}
$$

where $A=2 \pi / h^{2} m e$ and $f_{j}^{b}$ are the atomic form factors or scattering amplitudes for atom $j$, which depends on the atomic properties (scales as $Z$ ), the thermal vibrations of the atom, and the scattering angle. In eq $67, \Omega$ is the unit cell volume, $m$ is the electron mass, and $e$ the electron charge. The term $\varphi(\mathbf{k})$ is related to the diffracted intensity through the structure factor $\mathrm{F}(\mathbf{k})$,

$$
F(\mathbf{k})=\frac{2 \pi m e \Omega}{h^{2}} \phi(\mathbf{k})=\sum_{j=1}^{N} f_{j}^{B}(\mathbf{k}) e^{i \mathbf{k} \cdot \mathbf{r}_{j}}
$$

and has to be made more general to include the specific hkl Miller indices defining the particular crystal symmetry and unit cell parameters. From inspection, this relation gives the modulus of the lattice potential, whereas one measures diffraction intensities that are then proportional to the square of the structure factors $|F|^{2}$. The phase factor of the exponential term $\left(\mathbf{i k r}_{\mathfrak{j}}\right)$ in this equation leads to the inversion problem. The structure factors are the most important quantities in structure determination. As written, eq 68 considers diffraction from a 3$\mathrm{d}$ lattice structure. Therefore, the phase could be anything and this would weight the observed amplitude similar to a change in spatial relationship between atoms. For time-resolved measurements, as above, the observable is the differential detection of the change in structure factors in which both the excited and unexcited fraction of unit cells needs to be included. The excitation process involves random absorption events such that the observed diffraction pattern is just the coherent sum of the excited and unexcited unit cells, for instance,

$$
F_{\mathrm{on}}(\mathbf{k}, t)=\eta_{\mathrm{exc}} F_{\text {exc }}(\mathbf{k}, t)+\left(1-\eta_{\mathrm{exc}}\right) F_{\mathrm{off}}(\mathbf{k})
$$

The term $\eta_{\text {exc }}$ is the fraction excited, which can be measured independently. The challenge is to determine the structure factors $\left|F_{\text {exc }}(\mathbf{k}, t)\right|$ in the face of the unknown phase term weighting the diffracted intensity. In conventional crystallography, one normally samples a large number of different crystal orientations to fully sample reciprocal space and then use a model for refinement. The larger the sampling of reciprocal space, the more accurate this procedure will be, although it remains model-dependent. For femtosecond electron diffraction studies, there are limitations on the number of samples and problems with maintaining alignment of excitation and probe for crystal rotation. There are limited means of preparing different crystal orientations such that femtosecond electron diffraction (FED) generally undersamples reciprocal space relative to conventional crystallography. It must be born in mind that the objective is not to determine an unknown structure but rather changes in structure from a known initial structure (reactant state) to a known final (product state) structure. The known initial structure and known conserved terms in the formation of the product state provide sufficient information for a unique inversion, as a solution to the inversion problem (vide infra).

In this respect, the are a number of tests for convergence. The most commonly used version is the $R$ factor: ${ }^{168}$

$$
R=\frac{\sum_{\mathrm{hkl}}\left\|F_{\mathrm{obs}}(\mathrm{hkl})|-| F_{\mathrm{cal}}(\mathrm{hkl})\right\|}{\sum_{\mathrm{hkl}}\left|F_{\mathrm{obs}}(\mathrm{hkl})\right|}
$$

Here the sum extends over all hkl. $F_{\text {obs }}(\mathrm{hkl})$ are the experimentally observed structure factors, and $F_{\text {cal }}(\mathrm{hkl})$ are the calculated terms from a model. $R$ vanishes for perfect agreement, and typically accepted values for an accurate structure have $R$ values around $20 \%$ or lower.

Another measure is the Pearson correlation function, which has the advantage that it is not sensitive to exchange between $F_{\text {obs }}$ and $F_{\text {cal }}$ if one overestimates one or the other value. The Pearson correlation coefficient, $\gamma\left(F_{1}, F_{2}\right)$, is defined as ${ }^{169}$

$$
\begin{aligned}
\gamma\left(F_{1}, F_{2}\right) & =\frac{\sum_{\mathbf{k}} c_{1}(\mathbf{k}) c_{2}(\mathbf{k})}{\sqrt{\sum_{\mathbf{k}} c_{1}(\mathbf{k})^{2}} \sqrt{\sum_{\mathbf{k}} c_{2}(\mathbf{k})^{2}}} \\
c_{i}(\mathbf{k}) & =\frac{\left|F_{i}(\mathbf{k})\right|}{\bar{F}_{i}}-1 \\
\bar{F}_{i} & =\frac{1}{N} \sum_{\mathbf{k}}\left|F_{i}(\mathbf{k})\right|
\end{aligned}
$$


The terms $F_{1}$ and $F_{2}$ represent $F_{\text {obs }}$ and $F_{\text {cal }}$ in this formalism, and $N$ is the number of atoms per unit cell. Perfect correlation in this case is 1 , while -1 is perfect anticorrelation; 0 represents no correlation. This correlation function will be discussed below in terms of optimizing structures.

The above prescription is used for structure refinements of static structures. For time-resolved measurements, one knows the initial, and to some degree the final structure, causally connected through the structural transition. As recently demonstrated, this additional information is sufficient to uniquely invert diffraction data to structures even with limited sampling of reciprocal space. ${ }^{37}$ The accuracy improves with better signal-to-noise ratios (SNR) and more orientations, but the degree of accuracy can be assessed by varying the initial and final constraints. The procedure is to simulate diffraction for the initial structure and the (partially) known fully relaxed structure. The signal in the detector plane needs to be transformed. The intensity $(I)$ at each pixel position $(a, b)$ is calculated according to

$$
\begin{aligned}
I(a, b)= & \sum_{h k l} \exp \left(-\frac{\Delta q_{\text {radial }}^{2}}{2 \sigma_{\text {radial }}^{2}}-\frac{\Delta q_{\text {parallel }}^{2}}{2\left(|\mathbf{q}| \sigma_{\text {parallel }}\right)^{2}}\right. \\
& \left.-\frac{\Delta q_{\text {rotational }}^{2}}{2\left(|\mathbf{q}| \sigma_{\text {rotational }}\right)^{2}}\right)\left|F_{h k l}\right|^{2}
\end{aligned}
$$

$F_{h k l}$ is the structure factor for the reflection $(h, k, l)$, calculated from the X-ray structure, and using the parametrized electron scattering factors found in ref 170 . The scattering geometry is shown in Figure 5.2 of ref 37 and defined as

$$
\begin{aligned}
& \Delta q_{\text {radial }}=\Delta \mathbf{q} \cdot \Delta \hat{\mathbf{q}}_{a, b} \\
& \Delta q_{\text {parallel }}=\Delta \mathbf{q} \cdot \Delta \hat{\mathbf{k}}_{0} \\
& \Delta q_{\text {rotational }}=\Delta \mathbf{q} \cdot\left(\hat{\mathbf{k}}_{0} \times \hat{\mathbf{q}}_{a, b}\right) \\
& \Delta \mathbf{q}=\left(\mathbf{q}_{a, b}-\mathbf{q}_{h k l}\right)
\end{aligned}
$$

The values of the three $\sigma$ components in eq 72 are estimated from the orientation fitting and tested by trial-and-error to give a good qualitative agreement with the experiment. The effect of the finite beam size needs to be included as a Gaussian convolution. The resulting simulated diffraction pattern is optimized for the static initial structure to provide an excellent approximation to the experimental diffraction and experimental parameters. The time series of diffraction patterns is subjected to a minimization function that encodes all of the information about the experiment in terms of the experimental data and uncertainties, and prior knowledge about the structure and structural transition by adding penalty functions, which bias the refinement of the atom positions toward chemically reasonable structures and toward a continuous path between the initial and final structures determined by either X-ray diffraction or other means. Eq 72 gives the general construction of the function. Eqs 77 and 78 provide more detail:

$$
\begin{aligned}
& \left.f(\mathbf{x}, t)=\sum \text { intensity ratio diff (exp }- \text { theor }\right) \\
& +\sum \operatorname{dev} \text { bonded dist and angles from mean grnd state values } \\
& +\lambda \sum \operatorname{dev} \text { from linear path bet thermally equil structures }
\end{aligned}
$$

where specifically each diffraction spot's intensity ratio (IR) is determined to give the constraints

$$
\begin{aligned}
f(\mathbf{x}, t)= & \sum_{\text {spots }} \frac{\left|\mathrm{IR}_{\text {spot }}^{\text {exp }}(t)-\mathrm{IR}_{\text {spot }}^{\text {theory }}(\mathbf{x})\right|^{2}}{\sigma \mathrm{IR}_{\text {spot }}^{2}} \\
& +\sum_{\mathrm{b}=\text { bonds }} \frac{\left(r_{\mathrm{b}}^{\mathrm{ES}}(\mathbf{x})-r_{\mathrm{b}}^{\mathrm{GS}}\right)^{2}}{\left(r^{L T}-r^{R T}\right)_{\mathrm{b}}^{2}} \\
& +\sum_{\alpha=\text { angles }} \frac{\left(\theta_{\alpha}^{\mathrm{ES}}(\mathbf{x})-\theta_{\alpha}^{\mathrm{GS}}\right)^{2}}{\left(\theta^{L T}-\theta^{R T}\right)_{\alpha}^{2}} \\
& +\lambda \sum_{\mathrm{a}=\text { atoms }}\left|\mathbf{x}_{\mathrm{a}}-\mathbf{x}_{\mathrm{a}}^{\mathrm{GS}}\right|^{2}
\end{aligned}
$$

The first term in eq 78 describes the agreement of the theoretical and experimental intensity ratios (IR), which is weighted differently for each spot according to the experimental uncertainty, $\sigma \mathrm{IR}_{\text {spot }}$. The theoretical intensities are then calculated, taking into account the photoinduced change in an isotropic Debye-Waller factor and the ratio of excited species. $F_{h k l}(\mathbf{x})$ is the structure factor of the excited portion of the structure with Miller indices $(h, k, l)$ and, similarly, $F_{h k l}\left(\mathbf{x}^{\mathrm{gs}}\right)$ is the structure factor for the unexcited initial structure. The calculation of these structure factors is similar to the established method in time-resolved crystallography ${ }^{171}$ but using parametrized electron scattering factors. ${ }^{170}$ The remaining terms in eq 78 are penalty functions to act as constraints to guide the refinement to physically reasonable geometries. The second and third terms in eq 78 give restraints on the bonded distances and angles, respectively, where the denominators represent the uncertainties in these values, estimated as the differences between the initial and fully relaxed structures. This is the key feature of this approach. The details of the molecular structural transition that are known or invariant provide the additional constraints needed to find unique solutions. These terms effectively act as phasing centers or reference points to find the other phases by using the weighting factors through a leastsquares fit to find the best values. In this way, all atomic coordinates in an asymmetric unit cell can be efficiently handled and optimized for each time point to give full atomresolved dynamics. This procedure provides a robust method that can be automated for generating molecular movies of photoinduced structural changes and chemical reactions. This approach is similar to the solution of the gas phase problem described above (eq 59), where the first term describing the agreement between theory and experiment in the gas phase is simply replaced by the sum of relative intensity changes over all observable diffraction spots, and the stabilizing term, $\Omega$, is constructed based on the known structures for the thermal phase transition as given by the remaining terms in eqs 77 and 78. The regularization parameter of eq 59 is partially included in eqs 77 and 78 by way of the uncertainties (sigma values) in terms 1,2 , and 3 , with a free term $(\lambda)$ which must be chosen carefully as described in the Supporting Information of ref 37. The constraints do not rely on any level of theory per se but on the inherent knowledge of the initial and conserved components in the final structure. The important aspect is that the initial structure is known and that the final product structure is generally known in the study of photochemical processes. There are inherent correlations in the structure that connect these end points. Even in the case of bimolecular processes involving uncorrelated motions, there are known 
conserved bond lengths and bond angles in the passage to intermediate states. This information content within the limitations of SNR and sampled reciprocal space is sufficient to find fully converged, unique, solutions to photoinduced structural changes and reactions. One is exploiting the fact that the structures along a given time series after the excitation are causally connected to this perturbation. This additional information provides sufficient information for inverting to real space with a high level of accuracy without the need of theoretically predicted structures in the refinement process. The importance of this relatively recent advance needs to be fully appreciated as it is now possible to generate full atomresolved reaction dynamics in a model-independent way. This statement discounts that the conserved bond lengths, angles, etc. used as constraints are known to sufficient accuracy and are not construed to represent a model per se. The inclusion of additional information on the conserved structure relationships completely avoids the uncertainty normally inherent in the choice of models as well as sampling sufficient configurations in generating a structural response function to the excitation.

The development of new analytical tools that completely exploit known information on the system response will also enable the use of fewer electrons in the image reconstruction and open up the study of larger and more complex systems. We are far from the fundamental limit in extracting the maximum information from diffraction (e.g., see Walczak and Gruebmüller ${ }^{172}$ for a treatment of the minimum information needed for alignment of images). This topic is cited as a key area for further development that will be essential for pushing imaging of chemical reactions to the ultimate space-time limits in resolution.

\section{PATH TO ATOMICALLY RESOLVED REACTION DYNAMICS}

\subsection{Structures of Transient Intermediate States}

The first time-resolved electron diffraction (TRED) were limited in electron numbers per pulse and used long propagation paths to the sample, which inherently limited the time resolution to the subnanosecond range. This time resolution is sufficient to capture the atomic structure of reactive intermediates. In this respect, many important classes of such intermediates are photodark states that can not be studied by optical means to infer the structure. The general overall pathway including intermediates in the broadest sense of photochemistry is shown in Figure 10. The ultimate aim is to observe the correlated motions leading to the intermediate states and/or product state to give an atomic perspective of what are shown as arrows along speculative pathways involving approximated potentials, that is, to put real structural details on these constructs. To directly observe the atomic motions would enable the refinement of our current models to experimentally give the potential energy surfaces governing photoexited state processes. The path to achieving this vision of atomically resolved reaction dynamics will be put in chronological order below.

The first TRED experiments that laid the foundation for time-resolved structural studies were performed with multiphoton IR dissociation of $\mathrm{CF}_{3} \mathrm{I}^{21}$ The gaseous $\mathrm{CF}_{3} \mathrm{I}$ molecules were excited by a TEA $\mathrm{CO}_{2}$ laser. The multiphoton infrared excitation dissociates the $\mathrm{CF}_{3} \mathrm{I}$ molecules,

$$
\mathrm{CF}_{3} \mathrm{I}+n h \nu \rightarrow \mathrm{CF}_{3}+\mathrm{I}
$$

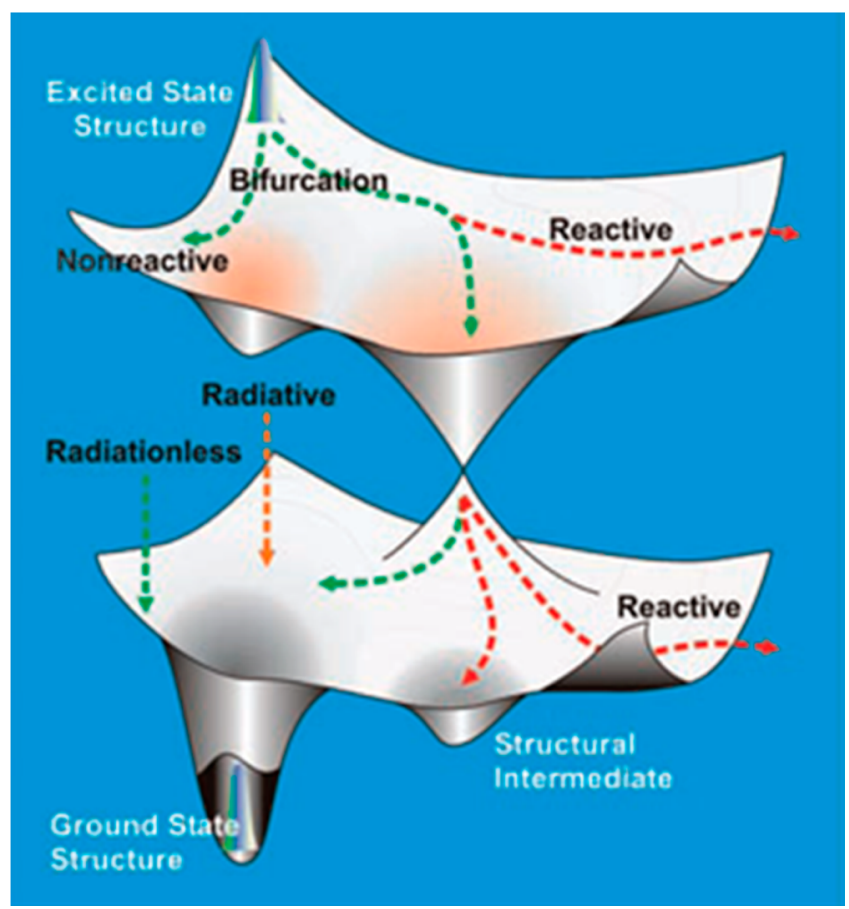

Figure 10. Landscape of the potential surface of the ground and excited states in complex molecular systems. The equilibrium structure of the ground state determines the initial wave packet produced by a femtosecond pulsed excitation. The excited state evolves either along a radiative or a nonradiative channel. Radiationless transitions may result in the bifurcation of a chemical reaction or physical process (internal/ intersystem crossing). Reproduced with permission from ref 212. Copyright 2005 American Association for the Advancement of Science.

and diffraction patterns of $\mathrm{CF}_{3}$ free radicals were recorded. For the experimental analysis used in refs 21 and 94, it was important that almost all of the $\mathrm{CF}_{3} \mathrm{I}$ molecules dissociated under the effect of a single $10^{-7} \mathrm{~s}$ IR laser pulse. ${ }^{173}$ This means that the scattering pattern was recorded from the bulk (inside the IR laser beam) containing no molecular components besides $\mathrm{CF}_{3}$, so that the resulting diffraction pattern was unambiguously associated with $\mathrm{CF}_{3}$ radicals. To record the diffraction pattern in time, an elegant method exploiting the use of a secondary electron multiplier, the pulse-resonance method, was employed to give an overall time resolution of approximately $1 \mu \mathrm{s} .^{174}$ This measurement of an unstable radical intermediate was a major advance as prior to this work we could only observe structures of stable long-lived radicals. These stable radicals are sufficiently complex in their own right that their geometric and electronic structures, cluster formation preferences, and reactivity have generally not been studied. ${ }^{175}$ Other techniques, including spin labeling and trapping, and EPR can provide important information about systems into which stable radicals have been introduced. The understanding of radicals is important both for their common role as reactive intermediates and for their spin properties. Efforts have been aimed at developing new materials with technologically relevant properties such as magnetism and conductivity for which stable radicals are attractive building blocks. ${ }^{175}$ Pioneering studies of persistent, long-lived radicals ${ }^{176}$ by gas phase electron diffraction (GED) were performed in 1968 by L. Schafer ${ }^{177}$ (please, see also ref 178). In these studies, a high-temperature nozzle tip was used to decompose diindenylcobalt, $\mathrm{Co}\left(\mathrm{C}_{9} \mathrm{H}_{7}\right)_{2}$, 

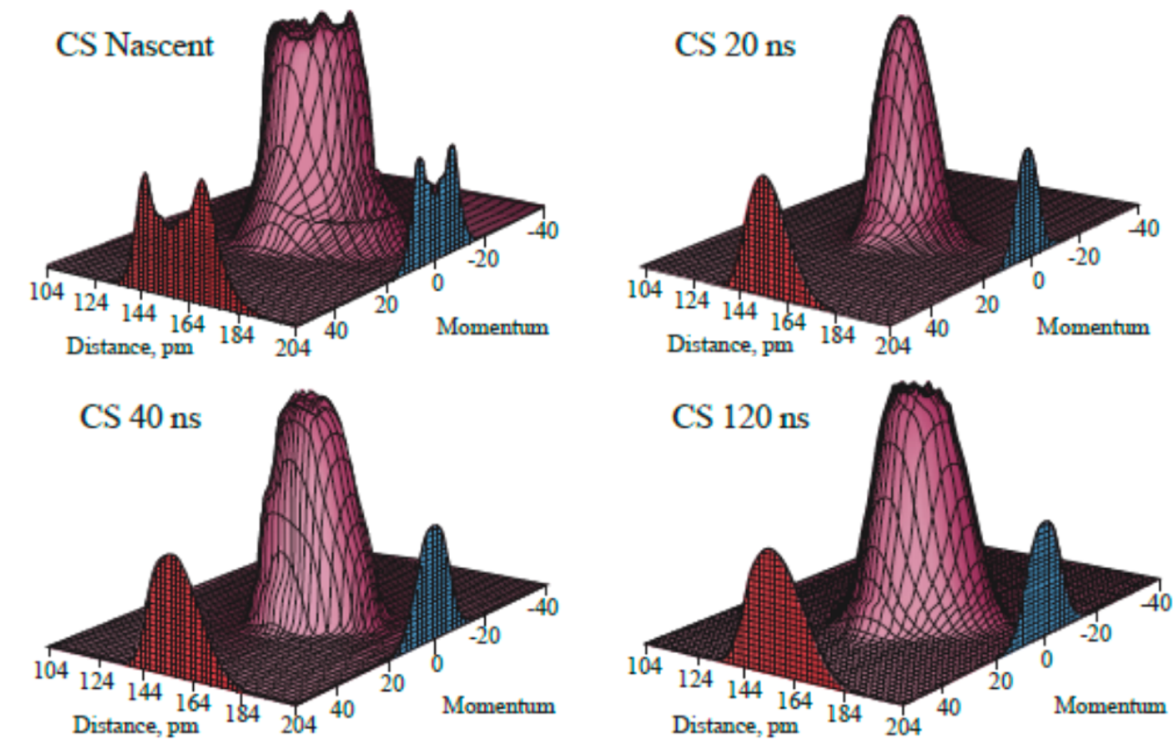

Figure 11. Wigner function for the CS photoproduct. For the initial distribution (within $\sim 1 \mathrm{ps),} \mathrm{the} \mathrm{Wigner} \mathrm{function} \mathrm{is} \mathrm{determined} \mathrm{using} \mathrm{the} \mathrm{full}$ density matrix. The Wigner function for the delay $\tau_{\mathrm{d}}$ of 20,40, and $120 \mathrm{~ns}$ were obtained by tomographic reconstruction using the procedure described in Ischenko et al. Reproduced with persmission from ref 128. Copyright 1999 Society of Photo Optical Instrumentation Engineers.

to produce indenyl radicals, $\mathrm{C}_{9} \mathrm{H}_{7}$. Schafer's experiment represents the first example of an investigation of a radical by GED to get complete atomic structures beyond that possible with ESR and spin-labeling methods. Other achievements in the study of persistent ${ }^{176}$ radical structures by GED are described in a review article. ${ }^{95}$ However, most radical intermediates do not live long enough for GED or ESR. Given the importance of radical chemistry and their transient nature, the TRED experiment ${ }^{21}$ illustrated the power of timeresolved structural studies.

The next systems to be successfully studied were the UV photoissociation of $\mathrm{CS}_{2}$ molecules, ${ }^{179}$ photofragmentation of $\mathrm{Fe}(\mathrm{CO})_{5}{ }^{25}$ and $193 \mathrm{~nm}$ photolysis of 1,2-dichloroethenes. ${ }^{180}$ These studies exploited pulsed electron sources based on photocathodes with picosecond pulses for photoinjection as introduced by Mourou et al. ${ }^{31}$ using a modified streak camera (please, see also refs 181 and 182). The seminal work of the Zewail group in the development of time-resolved gas phase electron diffraction studies particularly stands out in this period. They were able to achieve time resolution on the $10 \mathrm{ps}$ time scale in which the time resolution was limited by the electron pulses and intrinsic velocity mismatch. Given the low molecular density, weak diffraction, and associated low SNR, these were pioneering experiments at the time and would still be considered challenging experiments. This work has subsequently been extended over a period of 10 years to the study cyclopentadienyl radicals, ${ }^{183}$ refinement of the $\mathrm{Fe}(\mathrm{CO})_{5}$ intermediates, ${ }^{184}$ that have been extensively reviewed. ${ }^{185,186}$

Of the above first few systems, we will focus the discussion on $\mathrm{CS}_{2}$ as it serves as a test system for all the gas phase studies and the improvements that have followed. In this regard, the work of Ewbank et al., ${ }^{179}$ initiated investigations of the photodissociation of $\mathrm{CS}_{2}$ molecules followed by transfer of the energy to the vibrational energy of the resultant CS fragments. ${ }^{132}$ Upon photoexcitation with $193 \mathrm{~nm}$ photons, $\mathrm{CS}_{2}$ photodissociates via a nonadiabatic, two-channel mechanism:

$$
\mathrm{CS}_{2}\left(\mathrm{X}^{1} \sum_{\mathrm{g}}^{+}\right) \rightarrow \mathrm{CS}_{2}\left({ }^{1} B_{2}{ }^{1} \sum_{\mathrm{u}}^{-}\right) \rightarrow \mathrm{CS}\left(\mathrm{X}^{1} \sum_{\mathrm{g}}^{+}\right)+\mathrm{S}\left({ }^{3} \mathrm{P}_{\mathrm{J}}\right)+\mathrm{S}\left({ }^{1} \mathrm{D}_{\mathrm{J}}\right)
$$

The $\mathrm{CS}_{2}$ molecule in the excited state is quasilinear but has equilibrium internuclear distances that differ from those in the ground electronic state. ${ }^{187}$ The predissociation of $\mathrm{CS}_{2}$ is known from optical studies to take place within about $1 \mathrm{ps}$.

When $\mathrm{CS}_{2}$ is irradiated in a supersonic molecular beam at a wavelength of $193 \mathrm{~nm}$, the initial distribution of vibrational energy in the CS fragment is inverted for both the ${ }^{1} \mathrm{D}_{2}$ and ${ }^{3} \mathrm{P}_{2}$ channels of dissociation. ${ }^{188-190}$ The distribution of the vibrational energy with a low level of population at $n=0$ and $n \geq 10$ was described as bimodal ${ }^{189}$ or wide. ${ }^{188}$

The experimental results show that in the first $20 \mathrm{~ns}$, the system rapidly evolves toward an equilibrium that corresponds to the collisionless intermolecular transfer of vibrational energy (probably due to dipole-dipole interactions). During this time, a bimodal distribution in momentum space, and the distribution of interatomic distances, approaches a monomodal, more narrow distribution (Figure 11). In the interval for $\boldsymbol{\tau}_{d}$ from 20 to $40 \mathrm{~ns}$, collisional energy transfer begins to dominate over the collisionless process. Thus, changes between 20 and 40 ns relate to the conversion of electronic energy into vibrational energy via collisions with $S\left({ }^{1} D_{\mathrm{J}}\right)$ atoms and molecules $\mathrm{CS}\left(\mathrm{X}^{1} \Sigma_{\mathrm{g}}^{+}\right)$. Between 40 and $120 \mathrm{~ns}$, saturation of this process takes place. The evolving diffraction pattern in the time interval between 20 and $120 \mathrm{~ns}$ following the excitation of $\mathrm{CS}_{2}$ was the first observation of the transfer of electronic energy of one product of a nonadiabatic two-channel reaction to vibrational and rotational energy of the other reaction products using structural probes. ${ }^{127,132,133}$ The use of tomographic reconstruction discussed above illustrates the detailed information one can obtain on nuclear distributions of reactive intermediates.

\subsection{Molecular Alignment: Prospect for Improved} Structural Determination for Gas Phase Systems

The ability of gas phase scattering experiments to describe distributions of internuclear distances is limited by the random orientation of molecules in the gas phase. But tremendous progress has been made to align molecules in the gas phase, with prospects for molecules as large as proteins. ${ }^{191} \mathrm{~A}$ recent review by Chang et al. ${ }^{192}$ describes in detail the use of inhomogeneous electric fields for the manipulation of neutral 

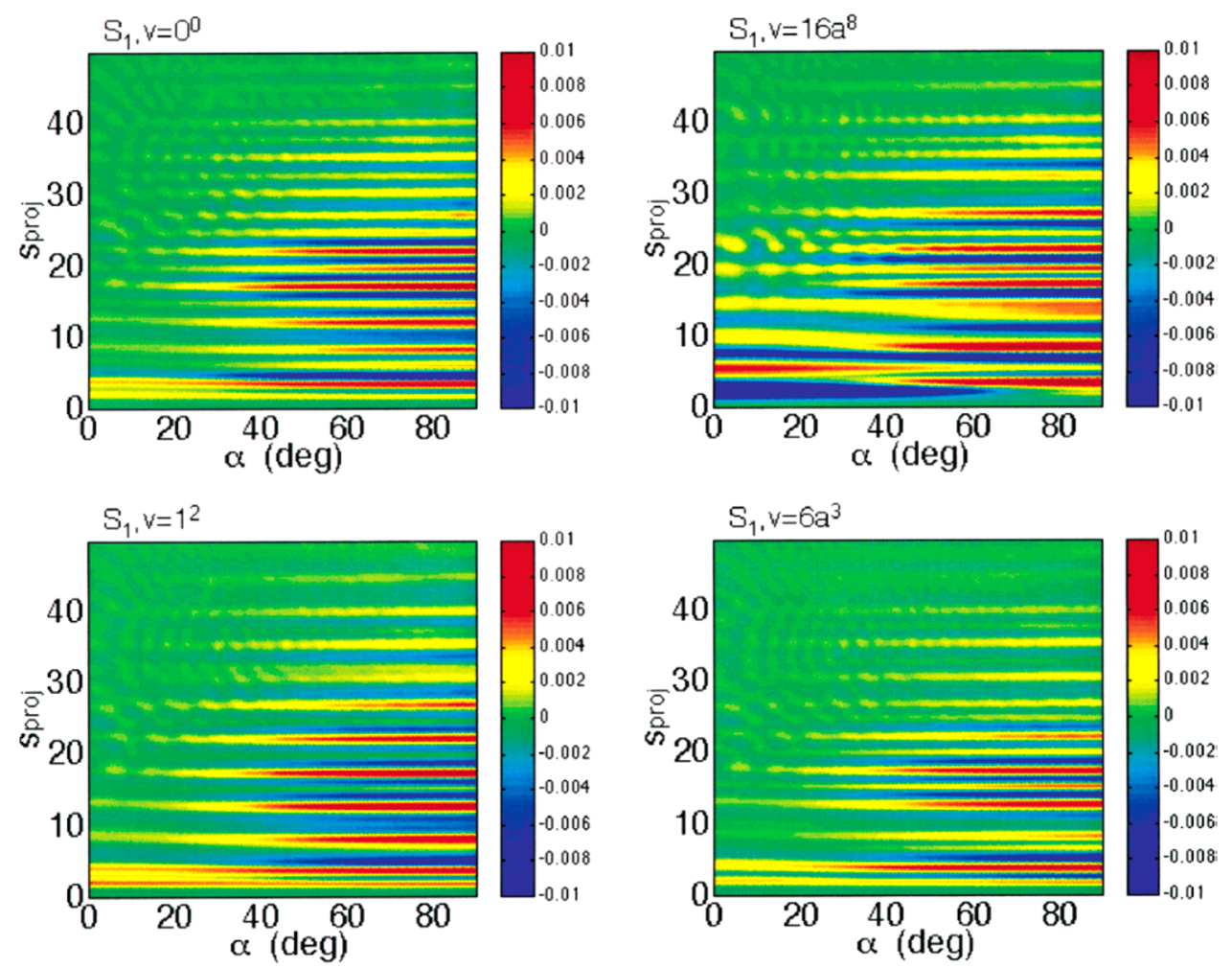

Figure 12. Diffraction patterns (difference between excited state patterns and the ground state patterns) of s-tetrazine, where a polarized laser excites the molecules of an isotropic sample to specific vibronic states. The abscissa gives the rotation about the electron beam axis, with the laser polarization at $\alpha=0^{\circ}$, and the ordinate is the projection of the s-vector onto the detector. Reprinted from ref 197. Copyright 2004 American Chemical Society.

molecules in the gas phase. For complex molecules, all quantum states are strong-field seeking, implying that dynamic fields are required for their confinement. Chang et al. discuss the current applications and interesting future applications. ${ }^{192}$

The most straightforward way to align molecules in timeresolved studies uses nonresonant laser pulses. The molecules are cooled by a free jet expansion to low rotational temperatures, implying small rotational quantum numbers. A strong nonresonant laser field then aligns the molecules with respect to the laser polarization. This mechanism involves changes to the rotational energy level structure that result in pendular motions about the polarization axis. ${ }^{193,194}$ This can be understood by considering the anisotropy of the molecular polarizability $\alpha_{\text {pol }}$. For example, for a diatomic molecule, the polarizability along the molecular axis is about 1.5-2 times larger than in the orthogonal direction. Therefore, when such a molecule is exposed to a strong external field from a laser, its potential energy depends on the molecular orientation, leading to the preferential alignment of the molecule in comparison to the laser field. It should be noted that the molecular alignment persists only for a limited period of time. Upon termination of the laser pulse, the alignment quickly dephases and the ensemble ceases to be quasi-one-dimensional. Under the certain conditions, the alignment is subsequently restored via rotational rephasing even without the laser pulse for field free conditions. A review of experimental and theoretical research in this area can be found in ref 195 .

Apart from use of differential polarizability for alignment, even a single absorbed photon using polarized excitation can induce a (partial) alignment of the molecular sample via photoselection. The diffraction signatures of aligned excited states of vibrating polyatomic molecules was discussed by Ryu et al. ${ }^{196,197}$ and by Weber et al. ${ }^{198}$ They calculated the patterns expected when the cyclic, 6 atomic aromatic ring molecule stetrazine $\left(\mathrm{C}_{3} \mathrm{H}_{3} \mathrm{~N}_{3}\right)$ is excited to specific vibrations in its electronically excited state $S_{1}$ (Figure 12 ). In the polyatomic stetrazine molecule, vibrational excitation leads to additional structure in the diffraction patterns because the vibrational probability density function (pdf) modulates the molecular structure.

Importantly, this modulation still manifests itself when isotropically distributed molecules in the gas phase are investigated: excitation with a linearly polarized laser beam induces a sufficient anisotropy to make the observation of the vibrational modes possible. Figure 12 illustrates this for two vibrational motions in the excited $S_{1}$ electronic state of stetrazine. Noteworthy is that the out-of-plane bending vibrations (mode 16a) have qualitatively different diffraction signatures than the in-plane stretch vibrations (modes 1 and $6 a)$.

The concepts summarized above for the s-tetrazine molecule in a far-from-equilibrium state are directly relevant to complex molecular structures where energy redistribution may or may not be complete. ${ }^{199}$

In terms of physical alignment, carbon disulfide, $\mathrm{CS}_{2}$, is a good system to test laser alignment concepts because of its large differential polarizabilities. ${ }^{187,200-209}$ Yang et al. explored the alignment of $\mathrm{CS}_{2}$ using electron diffraction ${ }^{210}$ and subsequently investigated the molecular dynamics upon excitation with intense $\left(10^{12}-10^{13} \mathrm{~W} / \mathrm{cm}^{2}\right)$ femtosecond infrared (IR) pulses. $^{211}$ It was possible to retrieve both the transient molecular structure and the angular distribution of the 


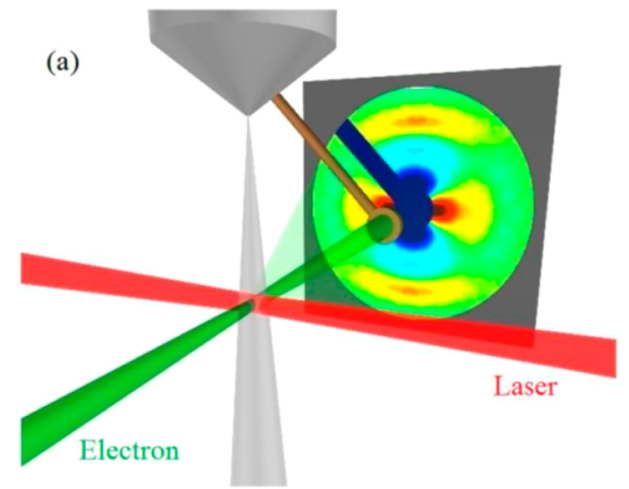

(b)

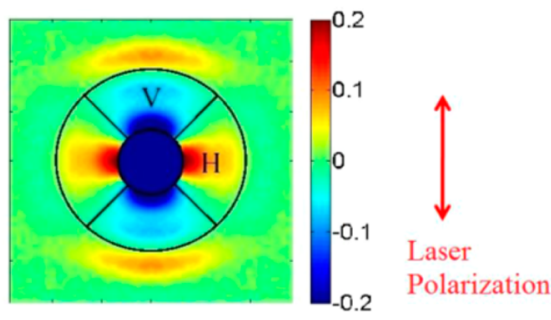

Anisotropy $=\mathrm{H}-\mathrm{V}$

(c)

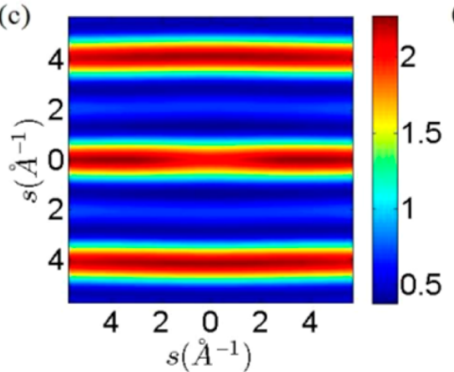

(d)

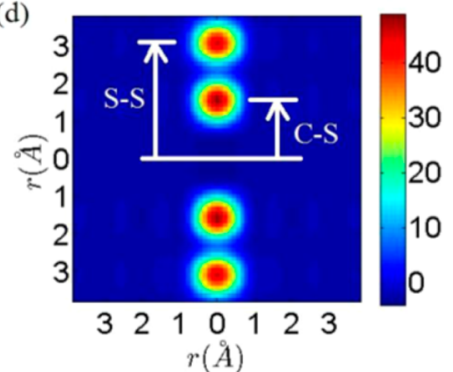

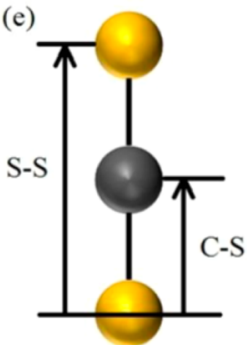

Figure 13. Experimental apparatus and method for recording diffraction patterns of aligned molecules. (a) The molecular beam, laser beam, and electron beam intersect orthogonally. A beam stop blocks the primary electron beam, while the scattering patterns are recorded by a phosphor screen that is imaged to a CCD camera. (b) The difference in total counts in horizontal $(\mathrm{H})$ and vertical (V) directions provides the anisotropy that is induced by the polarized laser beam. (c) The simulated diffraction pattern assuming perfect alignment. (d) An inverse Fourier transform of the simulated diffraction pattern gives the autocorrelation function of the molecule. Each signal spot shows an interatomic distance of the triatomic $\mathrm{CS}_{2}$ molecule shown in (e). Reproduced with permission from ref 211. Copyright 2015 Nature Publishing Group.

sample directly from the data without requiring theoretical modeling. ${ }^{191}$

To align molecules, laser pulses with intensities in the aforementioned range of $10^{12}-10^{14} \mathrm{~W} / \mathrm{cm}^{2}$ are often used. It should be born in mind that such pulses are capable of multiphoton ionization, ${ }^{205-207}$ and they can induce a broad range of dynamical phenomena. It is therefore interesting to explore their effect on the molecular structures, which is possible with time-resolved electron diffraction from aligned molecules (UEDAM). The conformational changes of molecules in the gas phase have been studied with TRED with a temporal resolution of a few picoseconds, ${ }^{137,212,213}$ while 3D static molecular structures were explored using UEDAM. ${ }^{214}$ If the molecules are probed after they interact with the alignment pulse (i.e., by using impulsive alignment), one can study them in a field-free environment. The electric field of the electron pulse is quite weak and therefore does not disturb the molecules. While the TRED experiments investigating conformational changes provide only $1 \mathrm{D}$ interatomic distances, $^{212,214}$ impulsive alignment yields $2 \mathrm{D}$ images of the molecules.

Figure 13 shows the experimental apparatus. The molecules are excited by an intense laser pulse with $800 \mathrm{~nm}$ wavelength, and the ultrashort electron pulse is used to record scattering patterns. The degree of alignment can be inferred from the anisotropy of the diffraction patterns (Figure 13b). To obtain a high quality diffraction pattern, about $10^{9}$ scattering events are accumulated.

The autocorrelation function of the molecular structure convolved with its angular distribution is the inverse Fourier transform of the diffraction pattern signal. ${ }^{210,211}$ For a perfectly aligned linear molecule, the image of the structure can therefore be determined from the autorcorrelation function. This is illustrated in Figure 13 (panels $\mathrm{c}$ and $\mathrm{d}$ ) for the case of a simulated pattern of the $\mathrm{CS}_{2}$ molecule. The internuclear distances in the molecule are imaged as bright spots in the autocorrelation image. At the center of the image is a spot that contains no structural information, but that is not shown in Figure 13 for clarity. For an imperfect molecular alignment, the inverse Fourier transform gives a $2 \mathrm{D}$ projection of the $3 \mathrm{D}$ image of the molecular ensemble, or an Abel transform ${ }^{215}$ along the direction of electron propagation.

The simulations described in Figure 13 show that the method is capable of measuring internuclear distances. For the ground state, distances of 153(3) pm and 311(3) pm are found for the $\mathrm{C}-\mathrm{S}$ and $\mathrm{S} \cdots \mathrm{S}$ bonds, respectively. The internuclear distances for the ${ }_{1} \mathrm{~B}^{2}\left({ }_{1} \Sigma_{u}^{+}\right)$state are $165(3) \mathrm{pm}$ and $324(3) \mathrm{pm}$ for the $\mathrm{C}-\mathrm{S}$ and $\mathrm{S} \cdots \mathrm{S}$ bonds, respectively, with a bent angle of $166^{\circ}$. The bending vibration has a frequency of $396.8 \mathrm{~cm}^{-1}$ and can be excited by an impulsive Raman process. ${ }^{216,217}$ The period of this vibration is shorter than the temporal resolution of the experiment and cannot be time-resolved in this experiment. Instead, it would lead to a broadening of the spot corresponding to the $\mathrm{C}-\mathrm{S}$ distance. ${ }^{210}$

This diffraction experiment with aligned molecules demonstrates a new way to study the dynamics of molecules. ${ }^{210}$ Using two laser pulses, one to align the molecules and a second to trigger the dynamics of interest, the method can be extended to study the reactions of photoexcited molecules. The method has several strengths and weaknesses when compared to laser-based spectroscopic probes. ${ }^{210,211}$ Advantages include (i) the molecular structure can be imaged with atomic resolution and no input from theory is needed. (ii) The probe does not perturb the target molecule. The limitations of the method are 
(i) it does not provide information on the electronic structure and (ii) given the complications arising from possible multiphoton ionization at the intensities required for alignment, there will always be questions regarding the nature of the excited state. This latter problem already arises in $\mathrm{CS}_{2}$, even though its highly anisotropic polarizability makes it easy to align relative to other possible molecular candidates. These complications will increase for less polarizable, larger molecules that require higher fields for alignment and that have multiple photoionization centers. Other means for alignment as discussed by Chang et al. ${ }^{192}$ still hold promise to use aligned molecules for structure determination.

\subsection{Scaling to Larger Systems: Electrocyclic Ring-Opening} Reaction of 1,3-Cyclohexadiene

The 1,3-cyclohexadiene (CHD) system serves as an important benchmark in extending electron diffraction to more complex organic systems relevant to understanding chemical processes. The light-activated ring-opening reaction exhibited by $\mathrm{CHD}$ (Scheme 1) is a prototypical mechanism that is invoked in

\section{Scheme 1. Photochemical Ring-Opening of CHD}
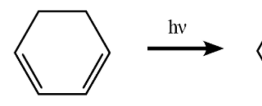

1,3-CHD

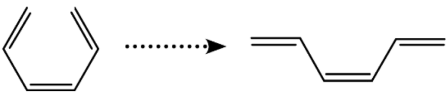

1,3,5-cis-hexatriene numerous more complex systems and has applications in optical control, optical switching, optical memory, light activated molecular machines, photobiology, photochromic materials, and conformation-specific photocatalysts (see refs in 218 and 219). In addition, CHD serves as an important system because of its relevance to synthetic organic chemistry and natural product synthesis. ${ }^{220}$ For example, the synthesis of vitamin $\mathrm{D}$ involves a ring-closing reaction that is the reverse of CHD's ring opening.

Deb and Weber ${ }^{218}$ and Arruda and Sension ${ }^{219}$ have recently reviewed the fairly extensive knowledge of CHD's photochemistry. The picture shown in Figure 14 is based on some 30 years of time-resolved experiments and computational studies. $^{221,222}$ The optical pulse excites the molecule to the FranckCondon region of the $1 \mathrm{~B}$ state, from where the wave packet rapidly slides past a conical intersection and down the well of the 2A state. At that point (labeled 2A/1A CI in Figure 14), the conrotatory stereochemistry of the reaction is already decided.

The system returns to the $1 \mathrm{~A}$ ground state via a symmetrybreaking displacement at the second conical intersection. From there, the molecule slides to either of the minima in energy (i.e., the initial state or the open structure of 1,3,5-hexatriene). CHD is an interesting system because the wave packet remains tightly confined during the reaction, sliding down the potentials in a ballistic fashion. ${ }^{223}$ This implies that "structure" remains a welldefined concept and is, in principle, observable throughout the reaction. On the other hand, the ring-opening of $\mathrm{CHD}$ does

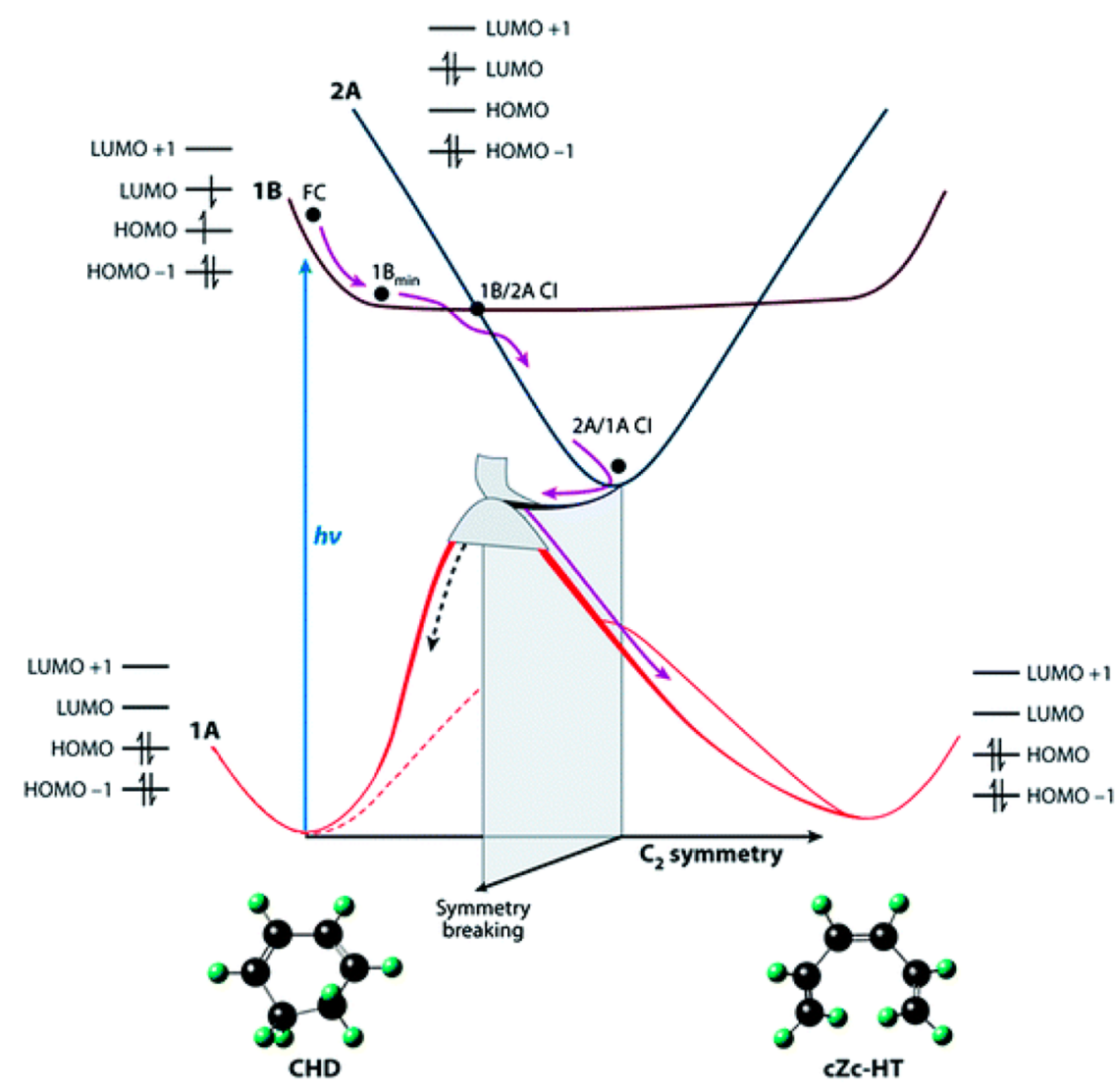

Figure 14. Ring-opening reaction of 1,3-cyclohexadiene (CHD). Upon excitation with a near UV pump photon (blue), the CHD molecule slides down the $1 \mathrm{~B}$ surface and crosses to the $2 \mathrm{~A}$ surface by avoiding a conical intersection that comes from above. Crossing to the ground state along a symmetry-breaking coordinate leads the molecule to the fully opened structure of hexatriene. Reproduced with permission from ref 218 . Copyright 2011 Annual Reviews. ${ }^{221,222}$ 
not lend itself to study the condensation of energy in a reactive coordinate as discussed above.

Structural parameters for ground-state CHD have been wellcharacterized from GED studies by Dallinga and Toneman, ${ }^{224}$ Trætteberg, ${ }^{225}$ and Oberhammer and Bauer. ${ }^{226}$ The first TRED studies of the electrocyclic ring-opening reaction of 1,3cyclohexadiene with few picosecond time resolution were published in 2001, carried out independently in the laboratories of A.H. Zewail ${ }^{32,227}$ and P.M. Weber. ${ }^{26}$ Changes in electron diffraction patterns upon irradiation of 1,3-cyclohexadiene with femtosecond laser pulses were observed on time scales of a few picoseconds. The time resolution was limited by the electron pulse duration and velocity mismatch between the laser photons and the electrons. As shown in Figure 14, when excited to its $1 \mathrm{~B}^{2}$ electronic state 1,3-cyclohexadiene undergoes a ring-opening reaction to hexatriene. ${ }^{228}$ From the saddle point, one pathway leads back to cyclohexadiene, while another path generates 1,3,5-hexatriene in one of its isomeric forms. The time resolution of the diffraction experiment was insufficient to capture the correlated motions through the CI. Ruan et al. suggested that after a few picoseconds, the ring-opening reaction results in hot structures with a (C5-C6) bond distance of over $170 \mathrm{pm}^{227}$ This is $\sim 20 \mathrm{pm}$ away from any expected equilibrium value, $r_{e}(\mathrm{C} 5-\mathrm{C} 6)=153.4(3) \mathrm{pm}$. Leastsquares refinement of the structural parameters ${ }^{227}$ were interpreted by Ruan et al. to be hexatriene (HT) molecular structures at each time slice. These HT structures revealed no tZt character but instead showed a configuration intermediate between $\mathrm{cZc}$ and $\mathrm{cZt}(\mathrm{t}=$ trans, $\mathrm{c}=$ cis conformer, and $\mathrm{Z}$ denotes ring open structure), far removed from a thermally equilibrated conformer distribution of $41 \% \mathrm{tZt}, 45 \% \mathrm{cZt}$, and $14 \% \mathrm{cZc}$ at an effective internal vibrational temperature of 2100 $\mathrm{K}$ (estimated from $\mathrm{ab}$ initio calculations of the conformer energies) (see Figure 15). ${ }^{199}$ On the basis of later studies with higher time resolution, discussed below, it is unclear if these transient excited state structures could possibly have been observed given the time resolution of these early experiments. Ruan et al. moreover suggested structures far from a thermally equilibriated conformation distribution, which persist for up to 400 ps. $^{227}$ This would imply a very slow vibrational energy redistribution within the product HT molecules. Typically, IVR leads to a statistical energy distribution on much faster time scales for a molecule of this size and associated vibrational density of states. It might be conceivable that the barriers separating the different conformations are so high as to prevent interconversion on the 100 ps time scale. While possible, there is no computational study that supports this explanation.

Despite many studies using ultrafast spectroscopies and TRED, many questions remain about the ring-opening reaction dynamics of cyclohexadiene. Deb and Weber pointed out that the spectroscopic methods cannot affirm that they are, indeed, observing the dominant part of the reactive wave packet. ${ }^{218}$ It might be that the spectroscopic methods are just more sensitive to a component that remains on the initially excited B state, while the dominant part of the wave packet undergoes the reaction through the $2 \mathrm{~A}$ state. In 2014, Minitti et al. ${ }^{164}$ published a pioneering article with the aim to settle this question and to determine the molecular structures as the reaction proceeds on a subpicosecond time scale. To achieve this, the authors used gas phase time-resolved X-ray diffraction (TRXD), with the CHD held in a gas bulb at very low pressure. This work was able to achieve a $100 \mathrm{fs}$ time resolution. Two diffraction orders were observed and simulated with high-level

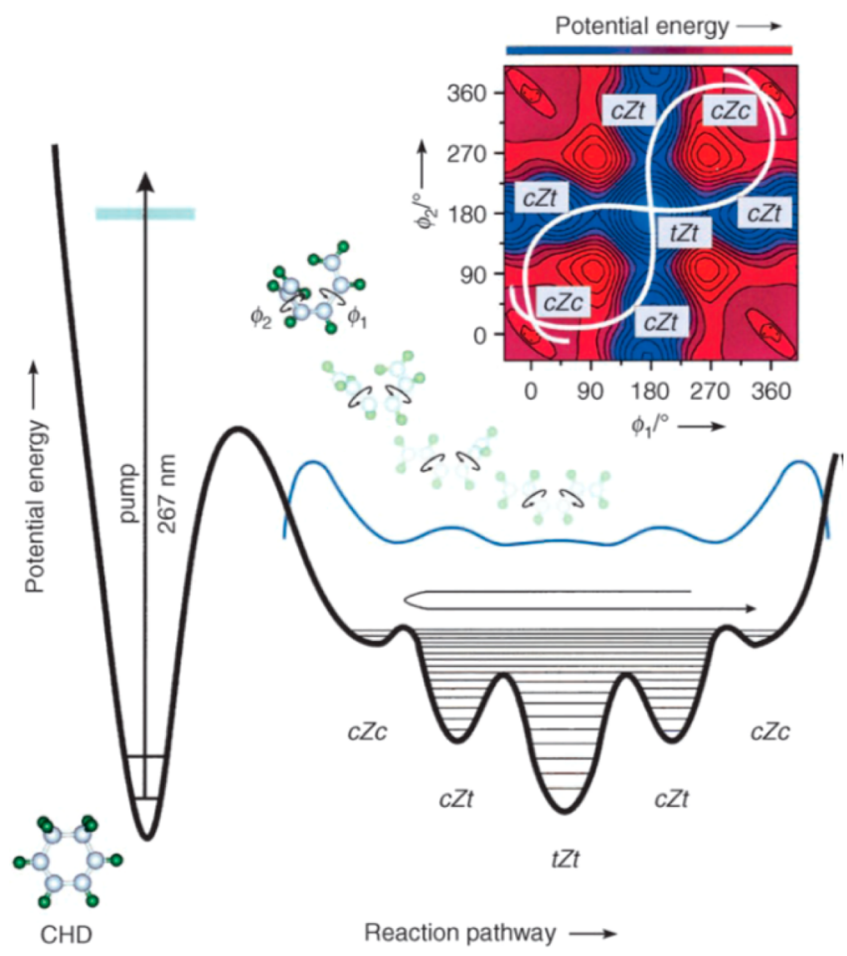

Figure 15. Potential energy landscape relevant to the formation of HT (see text). The schematically represented probability density distribution and potential energy curve of HT in its ground state, denoted with light blue and thick black solid lines, respectively, demonstrate the nonequilibrium nature of the reaction product. The increased probability densities in the vicinity of the classical turning points indicate the dominance of a near-cZc conformation, and the refined cZc-type molecular structure shown at one of the turning points is the statistical average, nonequilibrium structure obtained at $t$ $>400$ ps. Equilibrium structures of the $\mathrm{cZc}, \mathrm{cZt}$, and $\mathrm{tZt}$ conformers of HT are shown in pale colors. An isocontour plot of the ab initio potential energy surface $V=V\left(\phi_{1}, \phi_{2}\right)$ restricting the two internal rotational motions $\phi_{1}$ and $\phi_{2}$ in HT is shown at the upper-right. Possible trajectories leading to a time-averaged structure with a considerable $\mathrm{cZc}$ character are drawn with a white solid line. Reproduced with permission from ref 199. Copyright 2005 Wiley$\mathrm{VCH}$.

theoretical calculations of the trajectories. The experimental data act as constraints on the computational data. ${ }^{229,230}$ Because the ring opening leads to significant changes in the terminal $\mathrm{C}-\mathrm{C}$ distances, the two diffraction orders were sufficient to show the ring opening. Even so, many of the bond parameters with smaller changes could not be resolved. Higher time resolution experiments, using both electron and X-ray probe pulses, are in progress. It is clear that the femtosecondTRX experiments will soon provide a complete atomic description of this ring-opening reaction.

\section{BREAKING THE PICOSECOND BARRIER TO ATOMICALLY RESOLVED DYNAMICS}

As discussed in the introduction, the key enabling technology was the development of high brightness electron sources to get around problems associated with the need to phototrigger the reaction dynamics of interest and the generally irreversible nature of photochemical processes. The other essential issue is to use peak intensities of the pump pulse that are low enough to avoid multiphoton absorption that leads to ionization artifacts and loss of information on the initial state preparation. 
Due to velocity mismatch problems, save with relativistic electron sources, the highest time resolution is attained using solid state samples or with the use of recently developed nanofluidics for the study of solution phase dynamics. Typically, the experiment needs on the order of 10-100 diffraction patterns with integrated detection on the order of $10^{4}$ to $10^{8}$ electrons depending on the complexity of the molecular system and unit cell (small molecule/unit cell to proteins) for sufficient SNR. Condensed phase samples need to be approximately $100 \mathrm{~nm}$ thick (for $100 \mathrm{keV}$ electrons). This nanoscale thickness to avoid multiple electron scattering is highly nontrivial to prepare and only in limited supply. It becomes immediately apparent that it is the irreversible nature of the excitation process and strict requirements on the sample that are the biggest barriers to attaining atomic movies of reaction dynamics. There is no circumventing the sample requirements, and there are precious few samples that meet the additional requirements of barrierless photochemistry to track the key modes directing the reaction. The development of sufficiently bright electron sources to literally light up atomic motions in a single shot, to use the smallest amount of sample possible, solved these problems and can be viewed as the turning point that enabled atomically resolved dynamics.

\subsection{Development of Ultrabright Femtosecond Electron Sources}

Given the above sample limitations, it is clear that sufficient spatial resolution to resolve atomic positions and sufficient time resolution (100 fs time scale) to track the motions in time are not enough. In addition, one needs a sufficiently large number of electrons per pulse to attain atomic structures in a single shot. As discussed above, reaching the necessary source brightness is problematic considering the inherent electronelectron repulsion. Furthermore, it is imperative that the pulse duration, temporal instrument response function, and $t=0$ position be fully characterized to achieve the highest possible time resolution. This information effectively gives the shutter speed for image capture and allows each frame in a movie to be properly stitched together with the correct timing to capture the causally correlated atomic motions.

Once the brightness requirement was realized, daunting questions and challenges became apparent. How to generate electron pulses of $\sim 10 \mathrm{kA} / \mathrm{cm}^{2}$ peak current density on target with sufficient spatial coherence $(\geq 1 \mathrm{~nm})$ to enable near-single shot atomically resolved structures? How to fully characterize the electron probe pulses to determine the true time resolution at the sample position (with $<100 \mu \mathrm{m}$ accuracy due to the rapidly changing electron pulse profile) and sub-100 fs time resolution? Initially, there was no detector fast enough for the given beam parameters to make such measurements. Then there is the related question of how to determine the $t=0$ position between the laser excitation and electron probe pulse. The time origin is needed to synchronize the "movie" to correlate the temporal response and, equally important, provide essential information to correct for time jitter between the laser excitation and electron probe pulses. In addition, there are outstanding challenges with respect to samples, which is the ultimately limiting factor. How to fabricate samples sufficiently thin for electron transmission near the single elastic scattering limit that give high quality diffraction patterns? Since most chemistry occurs in the liquid state, is it even possible to obtain $100 \mathrm{~nm}$ thin liquid pathlengths to open up the study of solution phase chemistry? The liquid phase is generally thought to be the unique domain of X-ray scattering. There are equally important problems related to surface chemistry and heterogeneous catalysis. The extremely large scattering cross section of electrons make them the preferred probe of surface structure. However, low-energy electrons are generally needed to confine the observable to the surface plane. Brightness again becomes a major issue as the preparation of surfaces is very involved, and there is limited surface area. It is becoming abundantly clear that electron brightness is an essential consideration to open up all aspects of chemistry to atomic inspection as illucidated in Figure 3.

The above challenges all need to be taken in the context that sample limitations are the biggest challenge for this class of experiments, especially for solid state samples, which inherently give higher spatial resolution (assuming high quality crystals). Only in the case of gas phase studies is the sample not the limiting factor. For proper calibration and to state the problem succintly, one needs approximately a 100 fold more sample than for typical crystallography experiments and sample thickness must be on the order of $100 \mathrm{~nm}$ (to $1 \mu \mathrm{m}$ in the case of relativistic electrons). These requirements should give some calibration to the importance of source brightness. In all applications, it is better to use the brightest electron or X-ray source possible.

As will be seen below, all of the above listed technical challenges have now been solved.

High Brightness, Nonrelativistic Electrons. As discussed in the introduction, the key turning point in source development came from an effectively exact numerical solution to electron pulse propagation at the targeted bunch density for near single shot conditions. ${ }^{47}$ Referring back to Figure 4, the rapid evolution in the electron pulse parameters at relatively high bunch density is apparent. There are two solutions that jump out at you. One solution is to make the electron gun as compact as possible (Figure 4A schematic). The other is to deliberately exploit the remarkable linear chirp that naturally develops with nonrelativistic electrons (see Figure 4B, $T=2$ $\mathrm{ns}$ ). This calculation showed that it was possible to generate near perfect linear chirp without significant loss in transverse coherence or spatial resolving power, at the critical electron densities needed for single shot structure determination. A linear chirp is the simplest time-dependent spatial correlation to invert for pulse compression. This work stimulated a large body of theoretical studies on electron source coherence and experimental studies that have borne out these findings, as well as bridge this study to the large body of work done in particle physics for accelerator design. ${ }^{231-234}$ We now have very accurate numerical and analytical theories for calculating the electron pulse propagation dynamics, as well as experimental methods for full characterization of the electron pulses and $t=$ 0 position. This development was essential to advancing electron source brightness.

The first three technical challenges have been met. On the basis of the theoretical modeling, it was possible to reach a good compromise in electron pulse parameters to open up atomically resolved structural dynamics in the critical $100 \mathrm{fs}$ to 1 ps time window to directly observe atomic motions. The first machines to break the picosecond barrier used compact electron gun designs. ${ }^{28,51,53,235}$ This concept is now capable of $<100 \mathrm{fs}$ instrument response functions with up to $10^{9}$ electrons $/ \mathrm{cm}^{2}$ per pulse on target with sufficient transverse coherence to study unit cells up to $5 \mathrm{~nm} .^{50}$ This instrument concept can be improved by at least another order of 

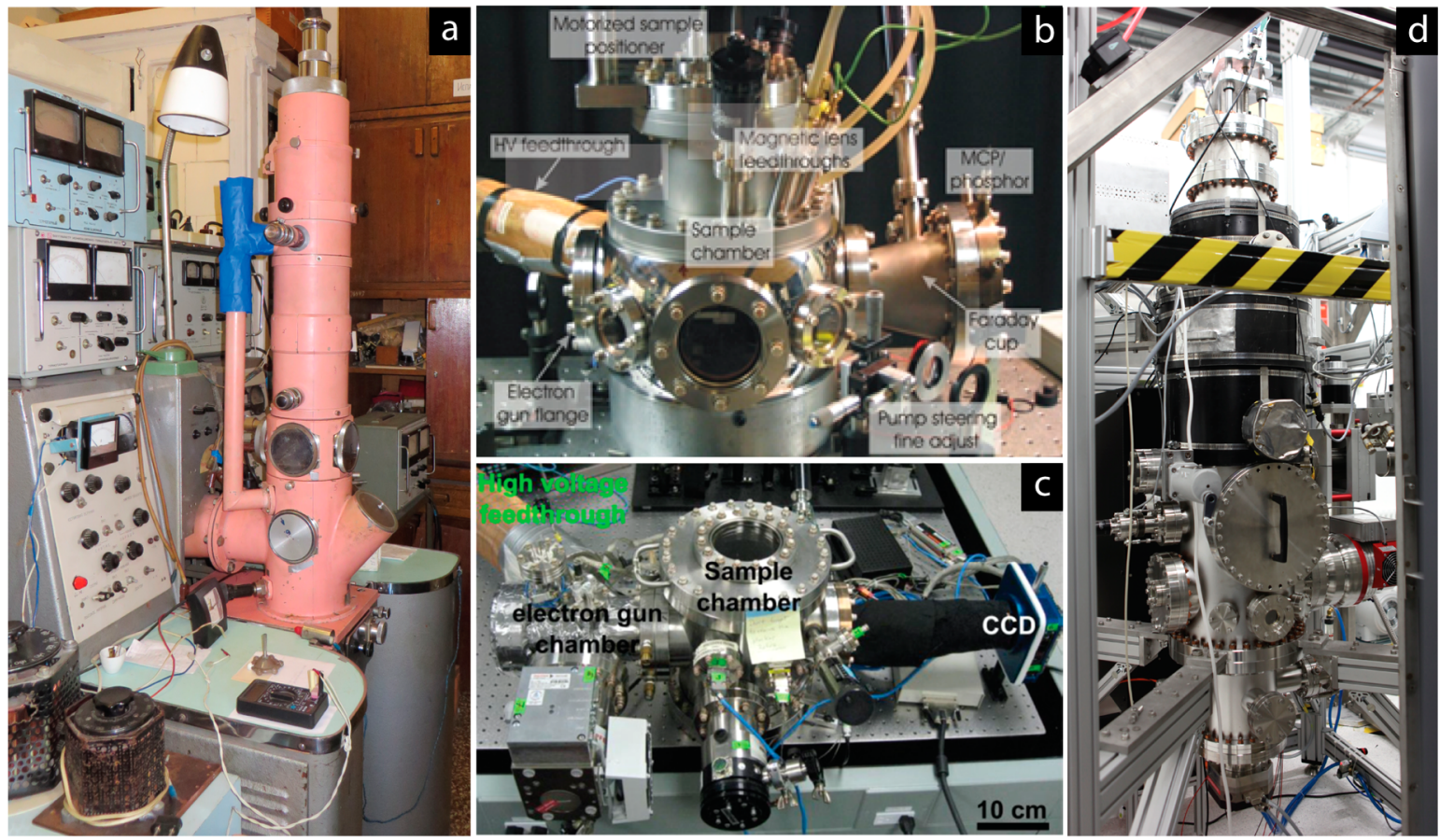

Figure 16. (a) The first time-resolved electron diffractometer at Moscow State University. ${ }^{21}$ (b) The 3rd Generation Compact Electron Gun (30 kV electron energies) at the University of Toronto, which was the first to achieve sub-picosecond atomically resolved structural changes. For scale, the dimensions are roughly the size of a soccer ball. (c) The 4th Generation Compact Electron Gun (60 kV, UToronto) resolved the fastest structural transitions at the time (e.g., < 200 fs nonthermal driven motion of Bi). ${ }^{33}$ Reproduced with permission from ref 247 . Copyright 2011 IOP Publishing. (d) Femtosecond Electron Diffraction Camera (FEDCAM, $300 \mathrm{kV}$ ) at the Max Planck Institute for The Structure and Dynamics of Matter, Hamburg. ${ }^{50}$ The size is comparable to a Transmission Electron Microscope.

magnitude in brightness with improvements in photocathodes $^{59}$ and is currently on par with other source concepts with respect to resolving structural dynamics for small unit cells $(<5$ $\mathrm{nm})$.

The next major advance in electron gun designs exploited the linear chip to compress the pulses. There are a number of possible electron optical systems that could compensate this chirp. ${ }^{55,56,236-239}$ A particularly elegant solution for high brightness, based on the findings of ref 47 calling for hybrid dc-pulse compression schemes, was developed by the Luiten group using a radio frequency (rf) cavity to compress the pulse. ${ }^{56,240}$ This hybrid dc-rf electron gun concept is similar to ref 238 for few femtoseconds pulse generation with the important difference that it was designed for high bunch charge parameters targeting the $100 \mathrm{fs}$ time window. In principle, this concept enables an increase in the electron bunch density by an order of magnitude over the compact gun design for beam conditions where the pulse broadening in the acceleration region (limiting factor for compact gun concepts) can be compensated. However, phase jitter between the rf and the excitation pulse used to trigger the structural dynamics has limited the instrument response time to a few hundred femtoseconds, ${ }^{57,240,241}$ so the effective increase in brightness over compact gun designs is only attained with some form of jitter correction. On the basis of this work, an rf pulse compression system has been developed at the University of Toronto with a magnetic lens system to provide the needed spatial coherence at the sample position and spatial point spread function at the detector plane. The electron pulse propagation dynamics and phase jitter for this system have been fully characterized, ${ }^{241}$ and the first results capturing atomic motions in the $100 \mathrm{fs}$ time domain utilizing the rf pulse compression concept have been demonstrated. ${ }^{35,57}$ The use of a zero jitter photoswitched streak camera has recently been demonstrated for time stamping and correcting the rf jitter to 30 fs. $^{58}$ This electron source concept is now capable of capturing even the fastest nuclear motions with single shot capabilities. The evolution in electron source technology is shown in Figure 16 to give some idea of the scale of the instrumentation and degree of sophistication. The overall trend has been to higher electron energies to increase the sample penetration depth to open up access to more systems and shorter pulses.

The current electron source technology has not reached its ultimate limit. The electron source brightness is determined by the source emittance (eqs 1 and 2). The closer the photon energy for photoemission is to the workfunction threshold for photoemission, the lower the transverse energy spread; or equivalently, the lower the emittance, the brighter the source, and the better the spatial-temporal coherence (eq 3). What are the fundamental limits to electron source temporal brightness? This question is really pertaining to the size of the object or complexity of the object to be imaged. As discussed above, in terms of following dynamics on the $1 \mathrm{~nm}$ scale pertinent to most organics systems, the compact electron gun is already operating effectively at the space charge limit to the bunch charge density. This limit delineates the onset to rapid Coulombic repulsion in the transverse direction and transverse emittance growth. With this qualification, the question reduces to whether the source brightness can approach similar charge densities for the study of systems as large as proteins. In other words, can the emittance be reduced to enable sufficient spatial coherence at optimal beam parameters for maximum bunch charge density? This question has been addressed using 
a
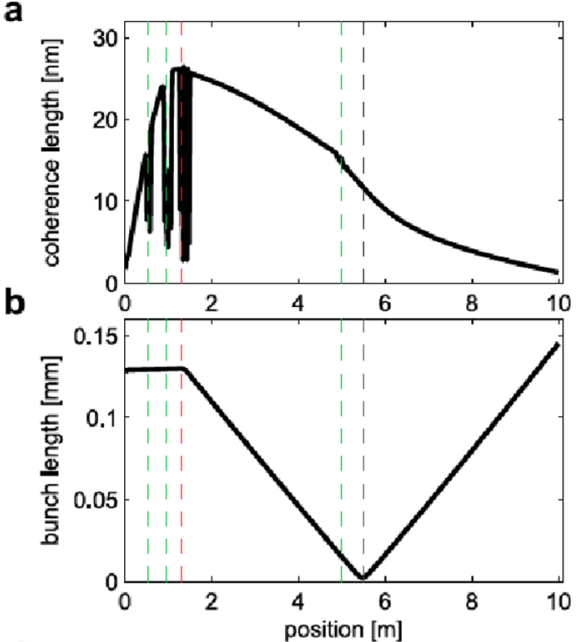

c

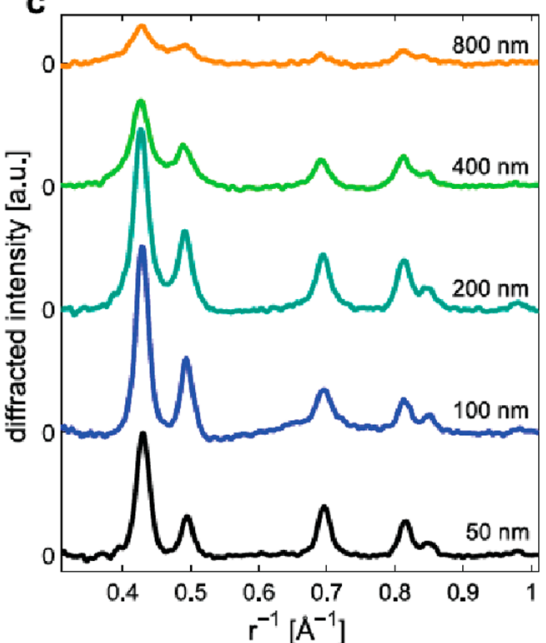

d

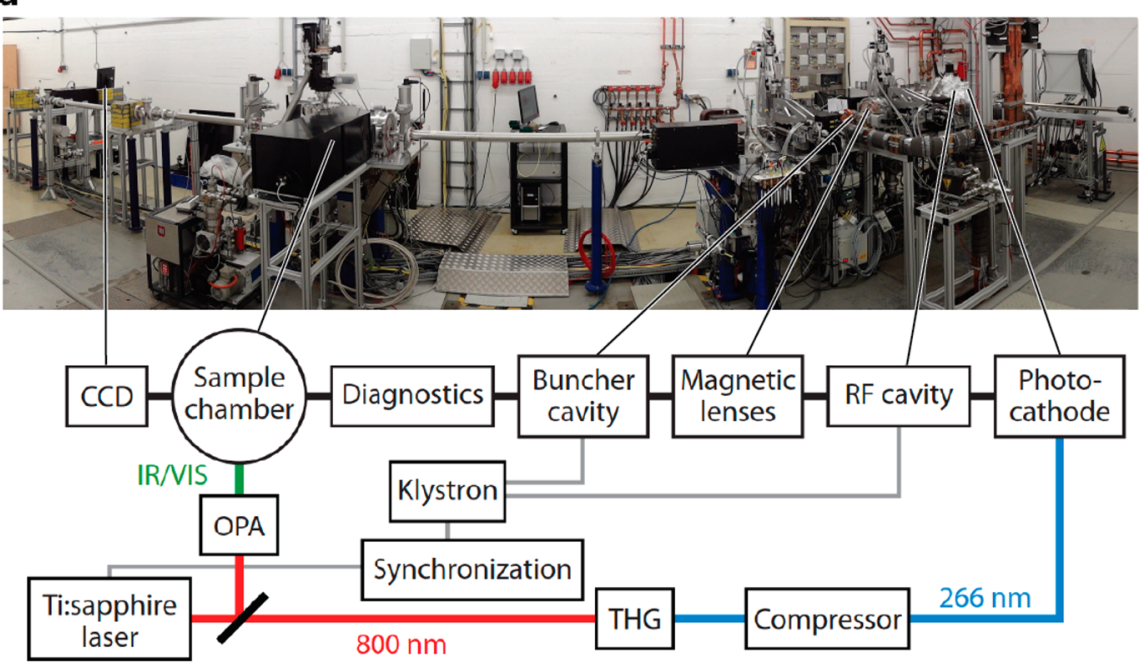

Figure 17. Relativistic electron gun for atomic exploration (REGAE). ( $a$ and $b$ ) The transverse spatial coherence and longitudinal profile (10 fs), respectively, at the sample position. The transverse coherence is sufficient for protein systems. (c) Diffraction as a function of sample thickness for polycrystalline Al. Samples for low Z such as organic systems can now be studied up to $1 \mu \mathrm{m}$ thickness. Adapted with permission from ref 69 . Copyright 2015 The Royal Society of Chemistry. (d) Photograph of REGAE with various elements labeled. For scale, the distance from the rf cavity to the sample chamber is approximately $5 \mathrm{~m}$. Adapted with permission from ref 2. Copyright 2014 Annual Reviews.

threshold photoionization of Rydberg states of ultracold atoms to serve as a highly coherent electron source, with a very nice account of these fundamental limits for nonrelativistic electron regimes. ${ }^{60,242-246}$ As bright as the electron sources are now, it should be possible to further increase the electron source brightness with respect to spatial resolution by at least 2 orders of magnitude. The ultracold atom sources are rapidly developing toward this aim. In parallel, there are very low emittance photocathodes being developed that have a factor of $>10$ lower emittance and with cooling could be further reduced to potentially compete with the ultracold atoms sources. ${ }^{59}$

Relativistic Electrons. The high-energy physics community has a long history in developing low-emittance electron sources for applications in particle physics and X-ray generation. ${ }^{248}$ However, the potential for using electrons for directly studying structural dynamics was not appreciated until femtosecond electron diffraction studies of strongly driven phase transitions ${ }^{28}$ demonstrated the importance of high bunch charge electron pulses to overcome sample limitations. The relativistic electron gun designs typically employ rf cavities to generate extraction fields that are an order of magnitude larger and correspondingly brighter than possible with DC extraction fields used for the current generation of nonrelativistic electron guns.

The first demonstration of a relativistic electron source for possible application in femtosecond diffraction studies was by Hastings et al. ${ }^{249}$ They showed a static diffraction pattern of polycrystalline $\mathrm{Al}$ obtained with a single subpicosecond electron pulse $(2.9 \mathrm{pC}, 5.4 \mathrm{MeV})$. This work was followed up by Musumeci et al. with a $3.5 \mathrm{MeV}$ source to show single shot capabilities. ${ }^{250}$ The time resolution was limited to 800 fs by rf timing jitter that can be improved by using actively phase stabilized rf, deflecting cavities, or time-stamping methods with time resolution now approaching $10 \mathrm{fs} .^{69,251-254}$ Tanimura and co-workers introduced a projector lens system after the sample similar to that used in commercial TEMs for diffraction mode imaging. The quality of the diffraction pattern is dramatically improved with time resolution estimated to be on the order of 100 fs. $^{255}$

The above sources do not take full advantage of the reduced longitudinal space charge broadening within the relativistic regime (all electrons travel near the speed of light). The residual chirp imposed by the rf acceleration needs to be compensated to reach the ultimate brightness. The relativistic 
a

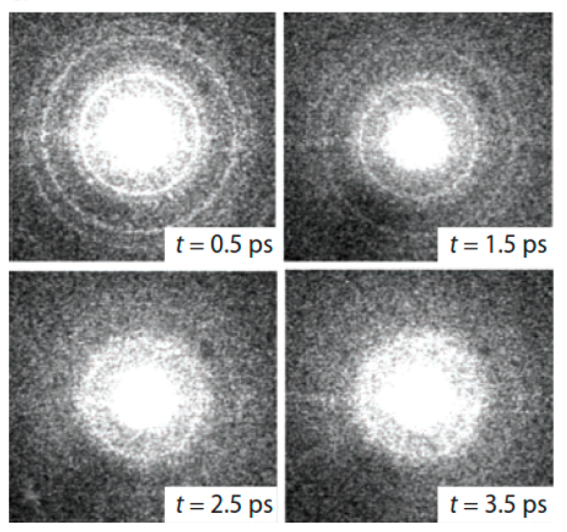

b

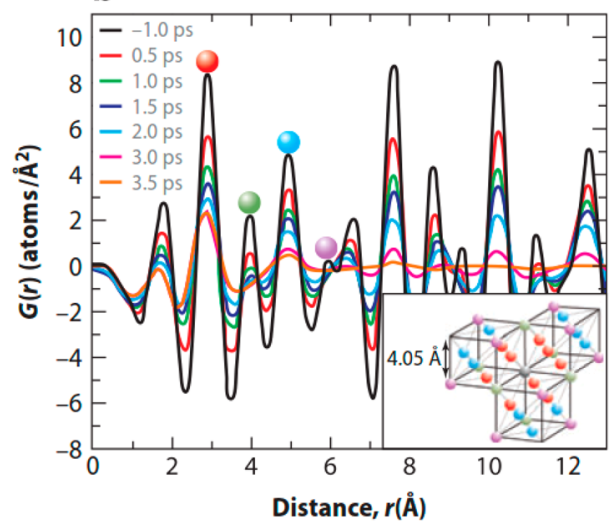

Figure 18. (a) The first time points for the strongly driven phase transition of polycrystalline Al. At $500 \mathrm{fs}$, it is clear the system is still in the fcc lattice, with a rapid rise in lattice temperature and reduced lattice coherence as evidenced by the reduced intensities in the diffraction at higher $q$ due to the Debye-Waller effect at $t=1.5$ ps. The lattice clearly collapses to a disordered liquid between 2.5 to 3.5 ps. (b) An atomic perspective of melting. The real space transform by which one sees the large amplitude motion of the corner atoms relative to the origin (i.e, the loss of the shear barrier to these large amplitude motions with the onset of melting). Adapted with permission from ref 2. Copyright 2014 Annual Reviews.

electron gun for atomic exploration (REGAE) developed in Hamburg in collaboration with DESY has solved this last hurdle to the highest possible time resolution. This system is unique in that it includes an rf rebunching cavity as a key design feature that compresses the electron pulses down to the $10 \mathrm{fs}$ regime, with transverse coherence $(>10 \mathrm{~nm})$ sufficient for protein studies. ${ }^{69}$ This development represents more than order of magnitude increase in time resolution and temporal brightness over previous work. This electron source concept uses the highest extraction field possible before excessive dark current and as such represents the ultimate limit to electron bunch density with current photocathodes.

This system is shown schematically in Figure 17. It produces well-resolved diffraction from samples on the order of a micron thick. The increased penetration depth associated with relativistic electrons opens up solution phase studies. In addition, the fact that the electrons are moving close to the speed of light eliminates velocity mismatch and loss of time resolution for gas phase studies. The user facilty at SLAC ${ }^{256}$ has recently demonstrated $100 \mathrm{fs}$ time scale resolution in the study of $I_{2}(g)$ excitation ${ }^{155}$ and direct observation of the excited state vibrational wavepacket. This work highlights the ability to resolve nuclear probability distributions for bound states, as previously demonstrated for reaction photoproduct distributions $^{127,133}$ (e.g., see Figure 11).

The main attraction of relativistic electron sources is the increased penetration depth for a given system and atomic scattering factors that enable the use of thicker samples, approaching micron dimensions. This latter feature makes relativistic sources comparable to femtosecond X-ray studies in terms of sample requirements with respect to using samples thin enough to avoid peak power limits and maintain $10 \%$ excitation levels to get above background.

\subsection{Realization of Atomically Resolved Reaction Dynamics: Imaging Chemistry in Action}

The simple process of melting, albeit under strongly driven conditions, was the first structural transition studied with sufficient diffraction orders to fully resolve atomic motions on the subpicosecond time scale required to catch the primary motions involved in the structural transition. ${ }^{28}$ This study involved the solid to liquid phase transition of $\mathrm{Al}$ in which the time resolution captured atomic motions faster than collisional processes could wash out the details. The importance of having sufficient diffraction orders is clear: if one examines only one order, it is not possible to dinstinguish nonthermal from thermal channels. The experimental results are summarized in Figure 18 with the real space transform and reconstructed first frames of this movie in Figure 19. It is important to note that the $600 \mathrm{fs}$ time resolution of this experiment was determined using a zero jitter streak camera after the sample and back projection to the sample position. This time resolution was essential to distinguish a very controversial point about possible nonthermal melting in $\mathrm{Al}^{258}$ which is an fcc lattice with no strong e-e correlations that would change upon excitation. By examining in detail the different diffraction orders, it was shown that the melting process was purely thermally driven, under the conditions of homogeneous nucleation. ${ }^{28,235}$ This conclusion was subsequently confirmed. ${ }^{259}$ As is apparent from Figure 18, the dramatic lattice collapse occurs within 1 ps (between 2.5 and $3.5 \mathrm{ps}$ ), which from simple speed of sound considerations indicates that the nucleation sites are limited in size to the order of 10 atoms (i.e., the length scale of the collapse of a unit cell within the solid state). The large amplitude atomic motions are outside the bound state of the lattice so that this observation witnesses the nearly simultaneous breaking of bonds as the $\mathrm{Al}$ lattice literally shakes itself apart. As a nice story of how basic science can lead to important real life applications, this new insight led to the development of a novel concept for completely avoiding run away nucleation growth and associated shock wave damage for laser surgery, finally achieving the fundamental (single cell) limit to minimally invasive surgery, without scar tissue formation. ${ }^{260-262}$

Subsequent work in the area has focused on photoinduced phase transitions. These include strongly driven phase transitions involved in nonthermal melting that create states of warm dense matter, to relatively weak perturbations of strongly correlated electron-lattice systems that give rise to interesting collective phenomena. ${ }^{33,34,263-265}$ By comparison, the first atomic movie depicted in Figure 18, in which bond breaking and increased rms motions to overcome the shear barrier to formation of the liquid state is reminiscent of the grainy early Deguerre type photographs. The quality of the diffraction increased dramatically to the point of generating 

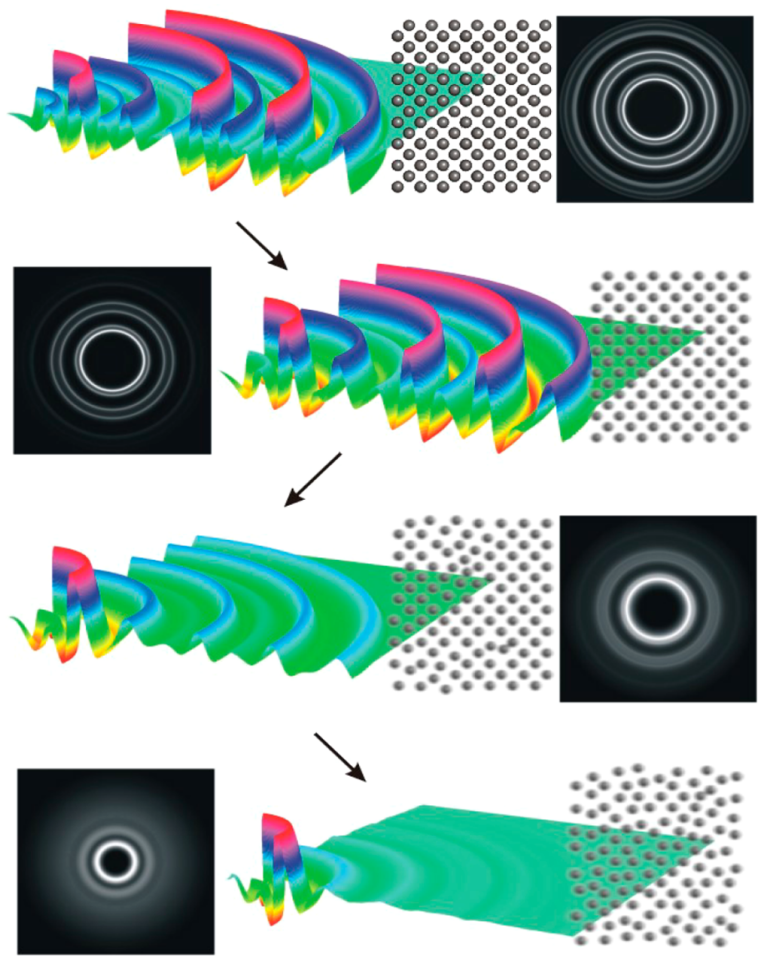

Figure 19. Dark panels showing diffraction are FT-filtered experimental data with the real space transform shown as the colorcoded radial plots (see Figure $18 \mathrm{~b}$ for spatial positions). The time course and representative atomic positions shown in the gray scale lattices. Top frame is the cold $\mathrm{Al}$ lattice before excitation, followed by time points showing from $500 \mathrm{fs}, 1.5 \mathrm{ps}$ in descending order showing increased lattice motion, to the final frame showing formation of liquid $\mathrm{Al}$ at 3.5 ps. Reproduced with permission from ref 257. Copyright 2004 University of Toronto.

patterns comparable to those obtained with high coherence TEM static sytems with the important distinction of femtosecond high brightness sources. ${ }^{34,255}$ Full atomic resolution with $100 \mathrm{fs}$ time resolution is now possible, yielding subtle details of electron-electron correlation effects leading to intriguing collective effects.

In parallel, there has also been remarkable progress with table top femtosecond laser generated X-ray plasma source that through a series of innovative developments by the Elsaesser group involving the use of very thin $\mathrm{Cu}$ targets, $\mathrm{X}$-ray collection optics, and use of thick polycrystal samples to increase diffraction efficiency have been able to resolve structural changes on the $100 \mathrm{fs}$ time scale with as few as $1000 \mathrm{X}$-ray photons/pulse. ${ }^{266}$ Recall the lower scattering cross section of $\mathrm{X}$-rays. This source is effectively $>10^{6}$ lower brightness in terms of scattering efficiency (per unit length of sample) relative to the electron sources discussed above. The use of powder diffraction was explicitly used to give all crystal orientations, which greatly increases data collection efficiency. The most relevant study with this approach was the work on the ionic crystal, ammonium sulfate, $\left(\mathrm{NH}_{4}{ }^{+}\right){ }_{2} \mathrm{SO}_{4}{ }^{2-} \cdot{ }^{266,267}$ It was possible to resolve changes in the electron density corresponding to a previously unknown concerted electron/proton transfer process. The excitation strategy in this case involved a nonresonant 3 photon process using $400 \mathrm{~nm}$ excitation (or $9 \mathrm{eV}$ ) to obtain a more uniform excitation within the relatively thick crystals (250 $\mu \mathrm{m})$ that better matched the X-ray probed volume. The peak power needed to get sufficient absorption to see structural changes above background in the diffraction pattern was on the order of $1 \mathrm{TW} / \mathrm{cm}^{2}$ (in the probed region), which is in the multiphoton ionization regime. The observed fraction of unit cells undergoing this effect was estimated from the diffraction to be more than an order of magnitude larger than the number of photons absorbed, taking into account the full crystal thickness sampled by the X-ray probe. There must be a highly nonlinear process involved. It was proposed that a very rapid energy loss of the 3-photon $(9 \mathrm{eV})$ excited electrons occurs to create hot polar phonons that in turn drive proton motion coupled to electron transfer from the sulfate anion as an explanation for the effect. There could be other explanations. For example, the ionization threshold of anions is generally less than $9 \mathrm{eV}$, and for the $\mathrm{SO}_{4}{ }^{2-}$ anion it is on the order of $6 \mathrm{eV}$ for ammonium sulfate crystals ${ }^{268}$ or a nonresonant 2 photon process such that multiphoton ionization may well dominate the excitation process. An alternative explanation is that the laser field drives the electrons photoionized from the sulfate anion to give impact ionization to amplify the effect. The laser field is $1 / 3$ the value leading to plasma formation typically at 10 $\mathrm{TW} / \mathrm{cm}^{2}$ via impact ionization, so this is a distinct possibility. In either mechanism, the process is a kind of chemistry in that charge is transferred; however, it does not involve localized anharmonic motions along a reaction coordinate that can be extended to understand reaction mechanism. In similar fashion, the bond breaking observed in $\mathrm{Al}$ discussed above is not chemistry per se but a collective solid state effect. One of the limitations in using this source technology to the study of chemical reaction dynamics is the requirement of multiphoton excitation for uniform excitation of thick crystals, which requires high peak powers to obtain a sufficient fraction excited to get above background scatter, as emphasized above. This requirement invariably leads to multiphoton ionization or other collective processes unrelated to the chemistry of interest. The work on the spin transition in $\mathrm{Fe}(\mathrm{II})$-tris(bipyridine) ([Fe$\left.\left.(\text { bpy })_{3}\right]^{2+}\right)$ is another example using multiphoton nonresonant excitation (2-photon at $\sim 1 \mathrm{TW} / \mathrm{cm}^{2}$ ) with the same approach. ${ }^{269}$ This work found a highly delocalized restructuring of the lattice involving up to $30\left(\left[\mathrm{Fe}(\mathrm{bpy})_{3}\right]^{2+}\right)$ and counterions, based again on the X-ray inferred fraction of unit cells involved in the structural changes relative to the fraction excited. This extensive restructuring was interpreted as lattice repolarization to stabilize the associate metal-to-ligand charge transfer character of the excited state. This work has since been repeated using very thin crystals and 1-photon resonant excitation at low peak powers into the well-defined excited state at this transition. ${ }^{270}$ In contrast, this latter study found that the molecular dynamics are not delocalized but rather highly localized and virtually identical to that observed in solution for definitively localized molecular dynamics. This finding indicates that the use of nonresonant multiphoton excitation led to photoionization processes in which case the extended lattice restructuring is understandable. This finding further underscores the importance of using 1-photon resonant transitions to well-defined states using samples thin enough to maintain peak power levels below ionization thresholds. This problem also pertains to recent XFEL studies, which used $\mathrm{TW} / \mathrm{cm}^{2}$ excitation conditions within the X-ray probed region to get sufficient changes in diffraction (re: shot to shot variations and partials), ${ }^{72,73}$ which brings to question the nature of the excited state and relevance to the photochemistry of interest. The main point to make here is that the laser-based Xray sources have achieved $100 \mathrm{fs}$ time resolution to structural 

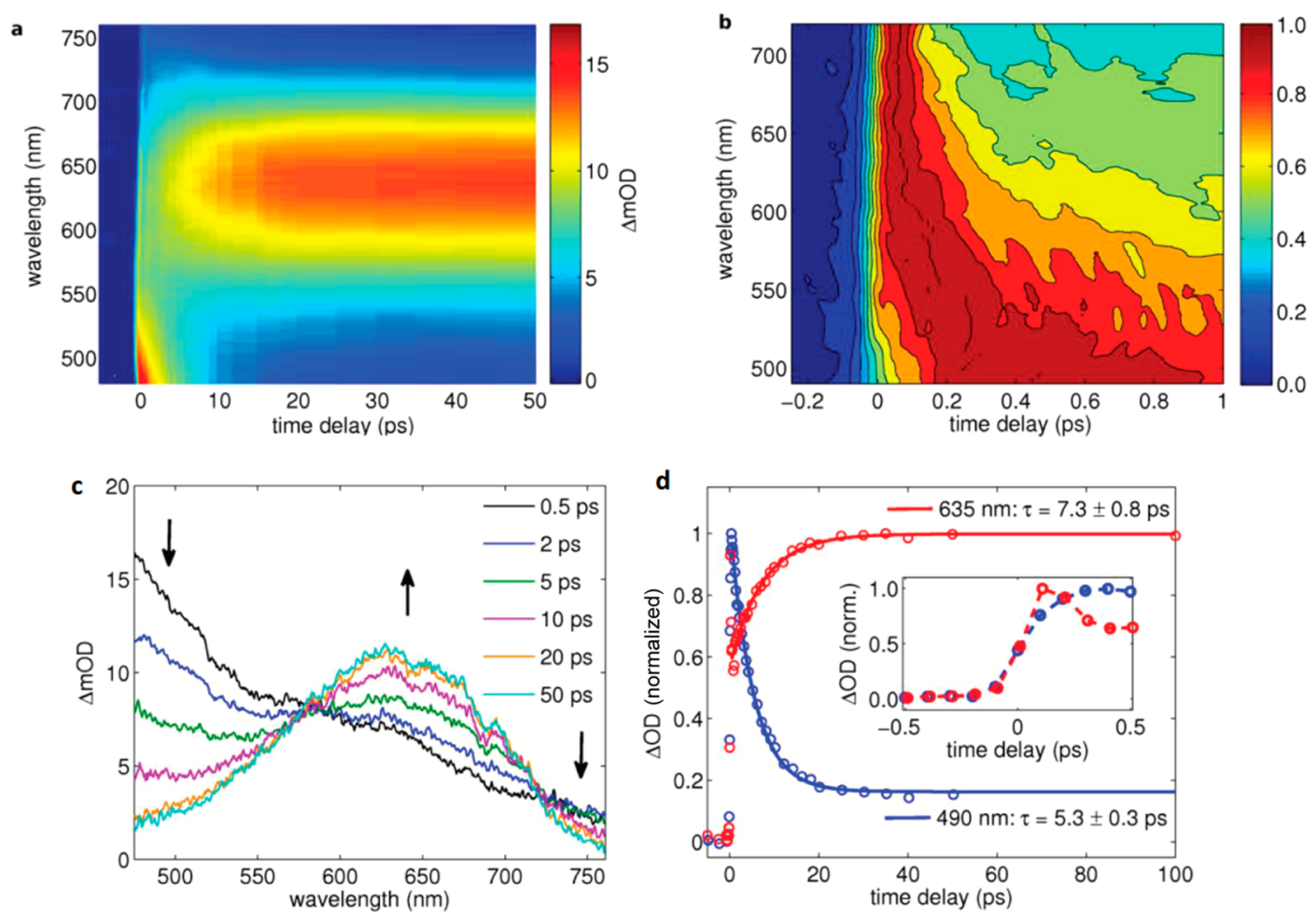

Figure 20. Femtosecond Transient Absorption Spectroscopy of single crystal diarylethene. (a) Femtosecond transient absorption spectrum of diarylethene ring closing dynamics out to $50 \mathrm{ps}$ to cover the full range of motions and vibrational relaxation. (b) Short time dynamics in which a 200-300 fs component is observed in the excited state dynamics, which can be assigned to low frequency flexing/torsional motion of the backbone of the molecular moiety to bring the labile carbon atoms into close proximity for wave function overlap. (c) Expanded view of the formation of the ring-closed photoproduct, which has a maximum at $635 \mathrm{~nm}$. There is continuous relaxation to the photoproduct state with time constants on the order of 5 ps as shown in (d). The slightly longer time constant at the photoproduct maximum is attributed to additional vibrational cooling. Adapted from ref 272. Copyright 2011 American Chemical Society.

dynamics. ${ }^{266,267}$ With further scaling in laser power, the source brightness should be sufficient to enable the use of 1-photon resonant excitation processes with thin enough samples to avoid peak power and multiphoton problems. In this limit, this laser based X-ray source concept will open up femtosecond structural studies of a wide class of materials.

From a chemistry point of view, the first molecular reaction captured with sufficient space-time resolution to follow the key reaction modes was the ring-closing reaction in the diarylethene system using femtosecond electron diffraction. ${ }^{35}$ The excitation exploited 1-photon resonances to well-defined excited state surfaces (see Figure $2 \mathrm{~b}$ for molecular structures of reactant/ product and potential energy surface). This system, like cylohexadiene, represents an important class of reaction mechanisms. As opposed to bond dissociation, this system involves bond formation and as such provides an important construct for understanding bimolecular processes leading to collision complexes and bond formation. It is the specific molecular structure of the diarlylethene construct that maintains the proper spatial relationship and minimizes entropic terms, to the attainment of the transition state for bond formation. This molecular moiety was developed through the pioneering work of Irie and colleagues ${ }^{271}$ to create photochromic switches. From a sample perspective, it is quite demanding to obtain sufficient number of $100 \mathrm{~nm}$ thin single crystal samples with sufficient surface area for typical laser excitation beam sizes. This seemingly trivial technical detail is actually the most challenging problem to overcome in extending femtosecond electron diffraction studies to the study of chemical reaction dynamics. In this respect, the reversibility of photochromic systems offers the prospect of signal averaging the same site to increase SNR and image contrast. The photoinduced ring-closing/opening reactions of diarylethenes are considered to be one of the most robust classes of photochemistry, capable of undergoing over $10^{4}$ photochromic cycles. This system undergoes relatively small changes in unit cell in switching between ring open (transparent, open state) to ring closed (opaque, closed state), which helps conserve the crystal structure. In this manner, the crystal strain upon photocycling is relatively small, which avoids strain induced lattice distortion and enables more photocycles. The integrated change in volume, small as it is, still leads to interesting actuator applications. Diarylethenes are considered to operate at the ultimate limit to photoreversible systems, essential to practical applications for photochromic switches. However, the changes in optical density only require a very small fraction of the total crystal for this function. As shown experimentally, at the high excitation fraction needed for femtosecond electron diffraction to get above background (10\%), even this system was limited in photocycles due to the competing nonradiative relaxation processes and accumulated heating effects. ${ }^{35,272}$ It was only possible to extend the number of photocycles to a few hundred by using an excitation pulse followed by light tuned to the photoproduct to drive the reverse process and avoid the buildup of lattice strain past the critical point. Even with this increased number of sampling events, the time to ensure complete photorecovery limited the repetition rate to $0.1 \mathrm{~Hz}$. Therefore, the SNR was greatly 

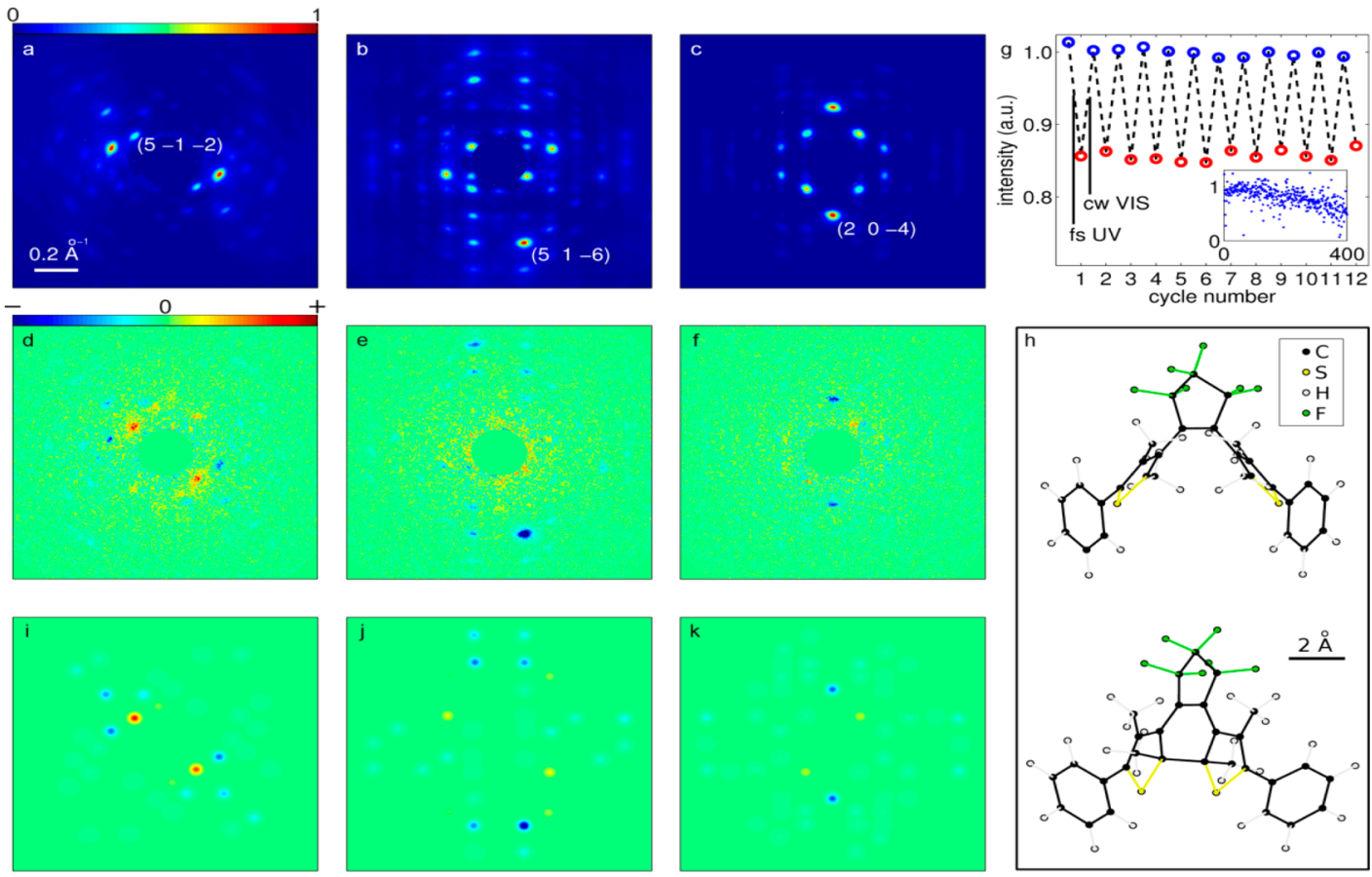

Figure 21. Femtosecond Electron Diffraction Studies of Diarylethene. (a-c) Static diffraction from different crystal orientations (assigned in comparison to calculated diffraction from the known structure with $R$ factors of $\sim 20 \%$ ). (d-f) Differential diffraction patterns following UV excitation to close the ring, after full relaxation for the same crystal orientations shown in $(\mathrm{a}-\mathrm{c})$. ( $\mathrm{i}-\mathrm{k})$ Corresponding calculated differential diffraction pattern for comparison, based on theoretically calculated changes in structures explicitly including the details of a singly excited molecule embedded within the ground state molecules forming the unit cell (see $\mathrm{h}$ ). The number of photocycles in which visible light was used to regenerate the initial structure was limited to the order 100 to ensure reproducible signal contributions (g). Reproduced from ref 35 . Copyright 2013 American Chemical Society.

limited by the laser noise in this sampling frequency range. The photorecovery step can be made to occur at much faster rates using higher intensity recovery pulses but this pumps more heat into the crystal. The low thermal conductivity of organic systems poses another limit, on the order of $10-100 \mathrm{~Hz}$ sampling, to avoid thermal accumulation and strain effects on the diffraction. There is a trade off in photorecovery rate and thermal effects on the crystal strain and diffraction quality. The required low-frequency sampling rate imposed by either thermal or photorecovery rates clearly demonstrates that this class of experiments requires high brightness sources to follow reaction dynamics in organic systems.

The exploration of the diarlyethene system, enabled by the high electron source brightness, is an important step toward understanding chemistry from a new perspective. First, it is an example of an electrocyclization reaction with conserved stereochemistry, a general reaction mechanism exploited in chemistry to create specific 3D architectures. Second, the process involves bond formation. As such, this particular reaction is a classic example of what would normally be a second-order, collision-mediated reaction. The fact that the reactive moieties are constrained by the intramolecular coupling and has withdrawing groups to soften a key double bond to guide the bond formation on the excited state surface is a brilliant synthetic strategy employed by Irie and colleagues. ${ }^{271}$

The photophysics of the excited state processes of the diarylethene system had been characterized in the solution phase to help identify the reaction dynamics. But it was not known in the crystalline state. This situation reflects the general situation in that photochemistry is often not well-characterized in the solid state. Most femtosecond spectroscopy has been conducted on solution phase systems for the ease of sample preparation and replacement. It is, however, essential for all solid state photochemistry of interest to determine the excited state dynamics using femtosecond single crystal transient absorption spectroscopy. These studies should be the first step in any considered problem. The structural dynamics observed using either electron or X-ray probes must match the spectroscopically observed components to ensure that the excitation processes involve the same states and to properly correlate the quantum state dynamics to the structural dynamics. This combined spectroscopic and structural information gives the full picture of both electronic and nuclear degrees of freedom. Femtosecond spectroscopic studies of single crystals are a nontrivial exercise due to sample damage issues that limit repetitive sampling and background scatter from imperfect optical surfaces. Apart from the work of Poulin et al. using an echelon for single shot dynamics with subpicosecond time resolution, ${ }^{273}$ the work of Jean-Ruel et al. opened up the study of molecular reaction dynamics in single crystals. Rather than using an echelon that effectively gives multiple pulses with fixed multiple time delays, it is possible to exploit the same sample preparation methods for electron diffraction studies (i.e., use multiple positions within a single crystal and multiple crystals to generate a database of thousands of shots to collect the dynamics with signal-to-noise rivaling solution phase studies). ${ }^{270,272}$ Again, these studies determine the excited state dynamics to ensure that the induced photochemistry is occurring from well-defined excited states and initial conditions. This experimental approach needs to be adopted universally both to characterize the operating excited state dynamics and to fully characterize the power dependence 

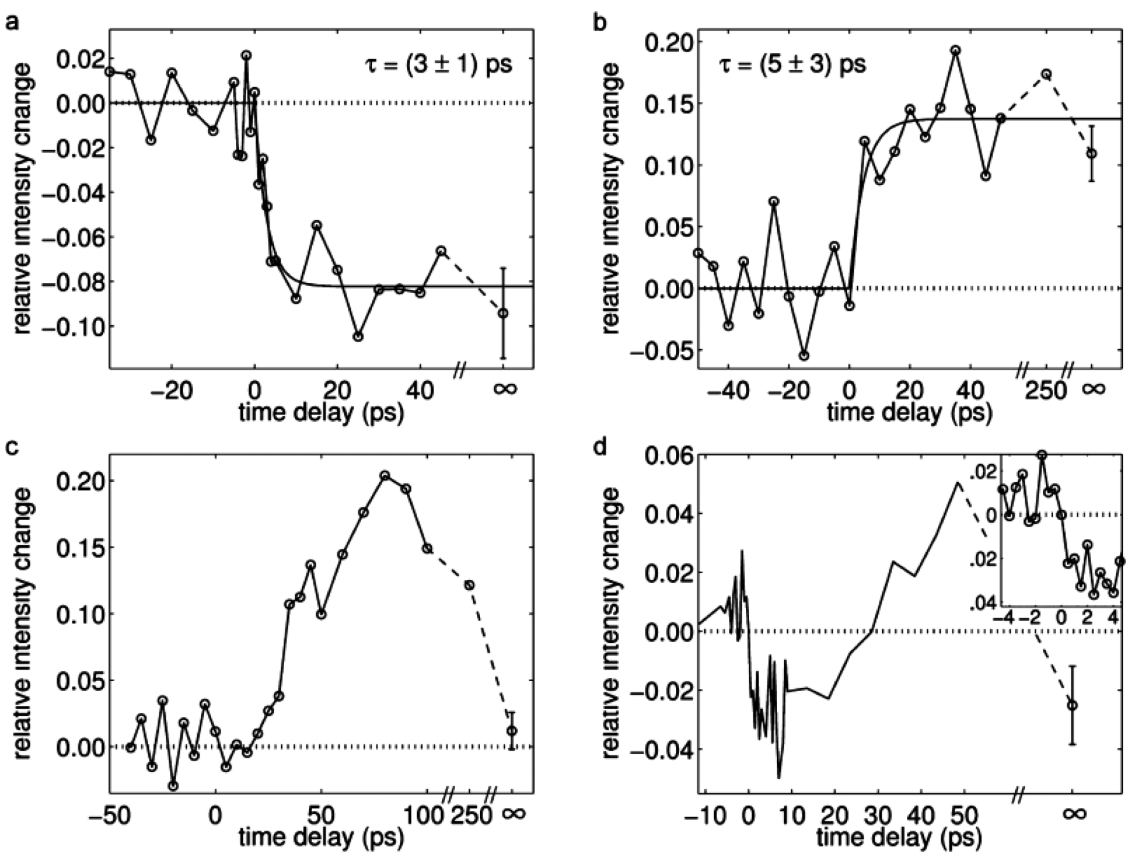

Figure 22. Femtosecond Electron Diffraction Studies of Diarlyethene. Time-resolved relative changes in diffraction intensity following photoexcitation for selected reflections. (a-d) Dynamics of Bragg peaks (5 1-6), (5-1-2), (2 $0-2)$, and (2 $0-4)$, respectively, averaged from a total of 18 samples. The subpicosecond dynamics are shown in the inset (d) for the (2 $0-4)$ reflection, clearly correlating the fast initial component observed in the femtosecond transient absorption studies to specific atomic motions. Reproduced from ref 35 . Copyright 2013 American Chemical Society.

to avoid multiphoton absorption and ionization artifacts. The optical studies illustrating the operating dynamics are shown in Figure 20.

There is a clear relaxation component on the order of 200300 fs with the final ring-closing process occurring within 5 ps. The fast relaxation component matches the $1 / 2$ period of the lowest frequency $55 \mathrm{~cm}^{-1}$ molecular backbone torsional mode involving rotation of the thiophene rings, as determined from DFT calculations. With a vector analysis of the relative motions, this mode would bring the two labile carbons into closer proximity for the conrotatary motion involved in the ring closing. However, the magnitude of the motion of this principal mode and the coupling to other modes resulting in the final bond formation can not be determined from the optical data. These studies do, however, provide the operating dynamics on the excited state surface. Equally important, the optical studies enable a laser titration of the power dependence of the response to ensure the excitation is free from nonlinear multiphoton processes. This power dependence is required for structural dynamics as there is always a temptation to use the highest possible excited state fraction (vide supra). In the diarylethene case, the peak power was kept at approximately 1 $\mathrm{GW} / \mathrm{cm}^{2}$ to avoid this problem.

The femtosecond electron diffraction studies that followed used, for the first time, the rf pulse compression electron pulse source for studying structural dynamics. This source generated high temporal brightness electron pulses with pulse durations less than $100 \mathrm{fs}$, transverse coherence of $\sim 1 \mathrm{~nm}$, with up to $10^{5}$ electrons per pulse ( $200 \mu \mathrm{m}$ beam diameters). The operational time resolution was approximately 200 fs due to timing jitter, and the dynamics did not require time stamping methods to attain higher time resolution to resolve the primary motions. The key results are shown in Figures 21-23 with an account of the methodology used to assign the structural changes. This work exploited time-dependent ab initio calculations using a hybrid imbedding method with different levels of ab initio theory for the reactive site and surrounding spectator molecules, developed by the Morrison group to specifically take into account the lattice. ${ }^{274}$ The lattice forms the intermolecular bath or solvent for the reaction and is comprised of unexcited molecules. The overall treatment involves timedependent $a b$ initio theory at the CASSCF level to properly deal with the breakdown in the $\mathrm{BO}$ approximation at the reactive crossing and DFT is used to treat the surrounding lattice with respect to structural minimization to obtain the lattice relaxation dynamics along the reaction coordinate.

The key motions involve an initial 300 fs relaxation component as observed in the femtosecond transient absorption spectroscopy (TAS) studies followed by slower, few picosecond components for different relative atomic motions. Earlier theoretical calculations predicted motions of the ring deformation on this time scale as part of the repulsive potential in the excited state to explain the efficiency of the bond formation and cyclization in diaryl systems. ${ }^{101}$ Given the highly truncated moiety to approximate the diarlyethene system, the exact magnitudes and even pathways are not well-defined at this level of approximation. The femtosecond electron diffraction studies were able to resolve the $100 \mathrm{fs}$ time scale, initial, inertial motions of the labile carbons with the softening of the $\mathrm{C}=\mathrm{C}$ bond linking the rings to increase the wave function overlap for bond formation (see inset to Figure $22 \mathrm{~d}$ for a representative example of the diffraction dynamics). The observed dynamics are in perfect agreement with the optical studies, as a necessary condition for ensuring the excited state dynamics are occurring within the linear regime for excited state preparation. The low-frequency flexing of the molecular moiety coupled to torsional motions of the thiophene rings is the key mode that directs the system to 

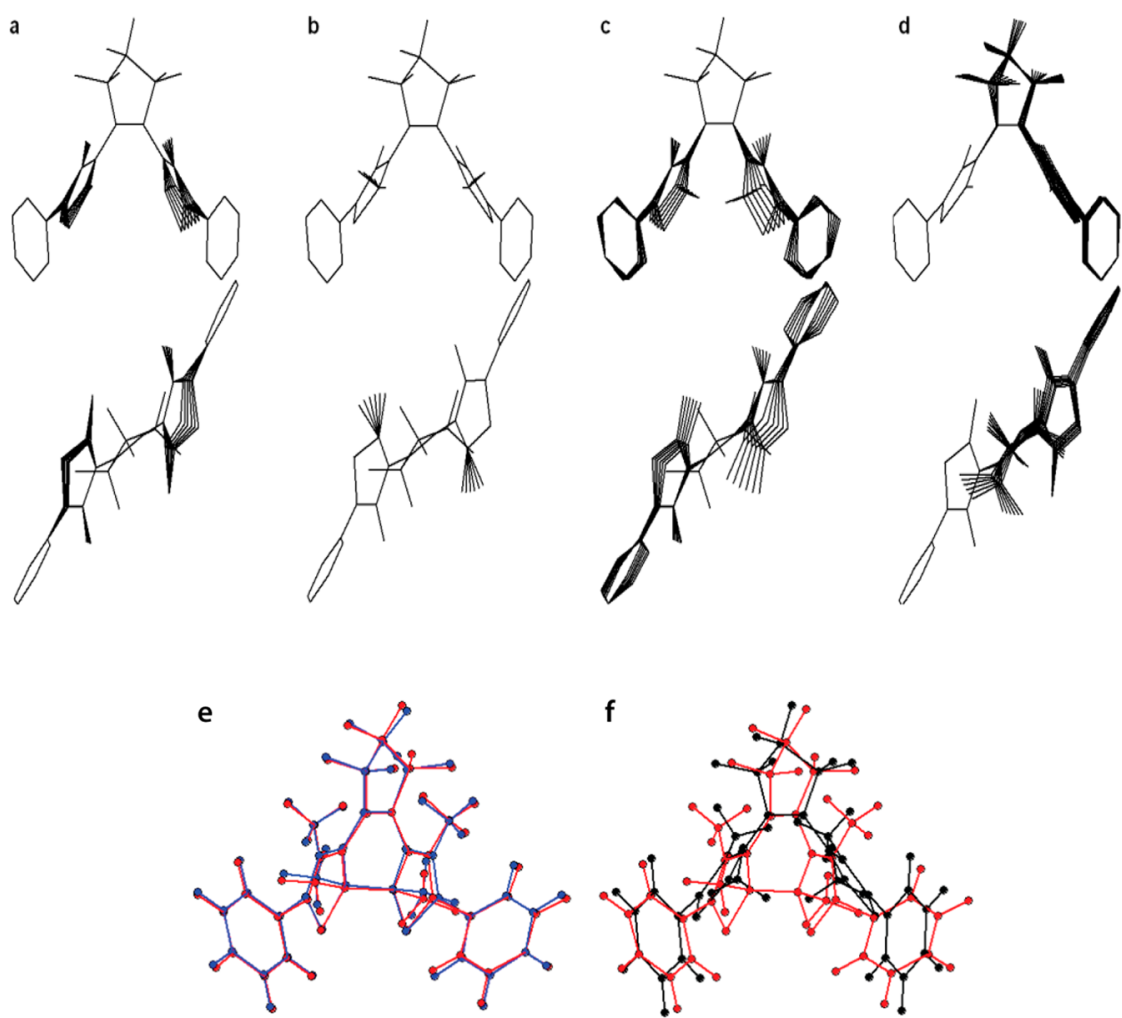

Figure 23. Ring Closing Reaction for Diarylethene. $(a-d)$ These are the 4 key modes found to fit the diffraction data to greater than $92 \%$ accuracy based on a Pearson correlation analysis. The main mode giving rise the fast initial motion in the repulsive excited state surface is mode (c), which creates a dynamic evolving potential gradient that drives the other modes. These motions are largely thermally populated by rapid IVR and undergo uncorrelated motion with respect to each other to give the closed ring form. (e) Comparison of the final photoproduct structure (red) obtained from the displacement of just these 4 modes in relation to the best theoretically calculated structure for the fully relaxed ground state ring closed form (blue). These motions clearly capture most of the important dynamics. (f) Net motions from ring open (black) to ring closed (red) based on the experimental results. Reproduced from ref 35. Copyright 2013 American Chemical Society.

the seam in the reaction coordinate. This displacement brings the two central carbon atoms involved in bond formation and cyclization into wave function overlap. One can now see how this process occurs sufficiently fast to block possible opposing torsional motions that would destroy the conserved stereochemistry for this process and any prospect of photoreversibility.

The implicated low-frequency mode is spatially delocalized over the entire molecule. How does the displacement of this mode lead to the highly localized motions needed to close the ring? This question has merit as it is not possible to formulate a simple picture for the motions involved or which modes may be most strongly coupled to the reaction coordinate given the strong degree of nonadiabatic vibrational coupling-an important lesson to come from the theoretical calculations of model systems for this problem.

Femtosecond electron diffraction studies answered this question. Indeed, there was a surprise, a common occurrence when observing such many body systems at the atomic level of detail. The results show that rather than going through what would be considered the classic transition state (which involves a barrier in the excited state surface as shown in Figure 2B from theoretical calculations of the excited state potential), there are higher frequency motions that couple the excited state surface to the ground state of the ring-closed photoproduct state. Using a combination of experiment and the level of theory discussed above, it was possible to cast out from the diffraction data a series of localized rotational motions that mix to produce the ring-closed form (Figure 23). The modeled trajectories and corresponding calculated diffraction patterns were compared through a Pearson correlation analysis (section 2.9, eq 71) to assign the specific structural changes in which convergence was found. Here the problem is not to determine an unknown structure but rather to take a known initial structure and from the diffraction data project out the pathways to the product structure. The information content in diffraction is enormous in comparison to integrated intensity values measured in spectroscopic measurements, which provide solely a single constraint and are not directly related to structure. Including all orientations and time points, there are hundreds of diffraction orders with necessarily spatially connected changes in intensity with time. The role of high level ab initio theory is essential as it provided the constraints in the relevant nuclear configuration space to find crystal orientations and provide a greatly reduced subset of solutions for the reaction dynamics. As discussed above, theoretical treatments of the isolated diarylethene ring closing, using a truncated molecular model, predicted a repulsive displacement (see Figure $2 \mathrm{~B}$ ) with the system moving toward a minimum in the excited state surface involving a displaced conical intersection in the reaction surface with a small barrier to ring formation. The predicted repulsive motion on the $100 \mathrm{fs}$ time scale along the reaction coordinate was born out by the experiment, and the key mode directing the ring closing ( $c$ in Figure 23) could be assigned, in approximate agreement with theory given the truncated nature of the model used. It was found with a high degree of confidence that the 
slower dynamics involve up to 3 highly localized torsional motions (Figure 23, panels a, b, and d), in addition to the lowfrequency torsional and deformation mode involving the thiophene rings. It is these modes that ultimately close the ring through a continuous relaxation process. There was, however, no well-defined intermediate structure indicative of a barrier in the excited state. It needs to be stressed here that the structural dynamics measurements are in excellent agreement with the femtosecond spectroscopic results and provide the missing structural information to explain the reaction pathways and associated dynamics. The effective continuous motions on the few picosecond time scale can be viewed as uncorrelated motions under a weak driving force without any specific intermediate. These motions were not specifically predicted from the theoretical calculations but were determined from diffraction data using the theoretical input as constraints.

This experiment was able to observe the formation of a chemical bond in which the hundreds of degrees of freedom condensed to just 4 key modes that led to cyclization. This observation of an extremely fast conversion of fluctuations to localized torsions explains how the stereochemistry is conserved. If there was one well-defined minimum or a distribution of minima then the system could thermally, via barrier crossings, explore the landscape and reach other spatial relationships with different rotations along the central axis. This exploration of nuclear configuration phase space would wash out the stereochemistry. However, the low-frequency deformation mode drives the system to a CI position in which the two labile carbon atoms can form a bond. In turn, this creates additional forces that lead to relatively rapid, localized, torsional motions at the central carbon bridge points. There are no significant minima or barriers leading to long-lived intermediates. The process is continuously driven to the closed ring form. This same coupling of vibrational modes and orchestrated localized motions at the point of bond formation would be expected as well for thermally driven cyclization along the ground state potential. We now see clearly how this process works. This point could have been conjectured, but the time scales involved and the coupling of delocalized modes and positions of the localized torsions provides new insight into controlling stereochemistry.

The ultrafast nature of the electrocyclization process, the snapping of the bond into place to close the ring, clearly separates the possible modes that are involved and highlights the importance of sufficient space-time resolution to connect the initial low-frequency mode to the localized torsion coordinates. See Movie S1 to get a better impression of the motions involved. This atomic level view of the cyclization process gives a remarkable perspective of chemical action leading to an enormous reduction in dimensionality of complex many body systems in the transition state region. It also highlights the key modes that are involved in directing this process and conserving stereochemistry that will help guide intuition in formulating new synthetic strategies.

The next system, within a systematic study of different reaction mechanisms, was ethylenedioxytetrathiafulvalene (EDO-TTF). ${ }^{36}$ This system undergoes a charge order phase transition in which thermally accessible configurations at higher temperature lead to electron transfer between thio-ring moieties to convert an organic system from an insulator to a metal. It is formally an intermolecular electron transfer process in the spirit of Figure 1. The "solvent" or bath in this case is the surrounding lattice molecules. Inspection of the changes in structure between the insulating and metallic structures would lead one to predict that the dominant mode coupled to the charge redistribution (intramolecular and intermolecular reorganization) would be the flattening of the slightly puckered rings at low temperature that would increase wave function overlap and lead to the formation of a conduction band. Given the near degeneracy of the orbitals at different positions of the EDO-TTF molecules within the lattice, it is possible to photoinduce the electron transfer process. The subsequent changes in structure that stabilize the electron redistribution were thought to involve a highly collective effect in which a very small fraction of intermolecular electron transfer processes would trigger the phase transition from insulating to metallic. ${ }^{275}$ This concept was based on the changes in optical properties monitored in reflection that appeared to be very similar to the high-temperature metallic phase. These studies used bulk crystals which, due to the extremely high absorption, necessitated time-resolved changes in reflectivity. The absorption cross sections can not be well-characterized in reflection without taking into account the entire complex dielectric response over a large wavelength range. It was believed the process involved very few excited sites within the lattice, and the effect was likened to a domino effect in restructuring the lattice. It is critical to know the degree of lattice excitation. Again, the ability to measure the femtosecond transient absorption spectra in the $100 \mathrm{~nm}$ thin crystals, as prepared for electron diffraction, enabled a determination of the fraction excited under the same conditions. ${ }^{36}$ These studies demonstrated that the structural changes involved with the photoinduced charge transfer were in fact localized, or molecular, rather than a long-range collective effect. The structural changes stabilizing the change in electron distribution truly represents an intermolecular electron transfer process.

This picture was further reinforced by femtosecond electron diffractrion studies that give a direct observation of the structural changes accompanying the photoinduced electron transfer. The study again used the same rf pulse compression system discussed above for generating the femtosecond electron pulses. With this high brightness electron source, the quality of the diffraction data was extraordinary, with hundreds of diffraction orders, well beyond $0.2 \AA$ in a single shot. To put this degree of reciprocal space in context, with $\mathrm{X}$-ray sources, it is typical to only be able to observe well-resolved diffraction orders out to $1.5-2 \AA^{-1}$ for complex organic crystals to remain marginally at atomic resolution (taken to be $2 \AA$ ). The diffraction for this system is more than an order of magnitude better and translates to exceptionally high spatial resolution. Here it must also be emphasized that these experiments were conducted at the same excitation levels as the optical experiments for direct comparison and to ensure low enough peak power to rule out multiphoton absorption artifacts. The quality of the differential diffraction patterns (with and without excitation) in light of the very low lattice excitation $(<10 \%)$ is further testimony to the importance of using high brightness electron sources.

The structural dynamics were determined by creating a basis of possible atomic motions connecting the initial and final structures using the well-characterized structures for the insulator and metallic phases. The static differential diffraction pattern can be obtained for this reference by simply scanning the temperature across the charge order phase transition. With this information, it is possible to interpolate the possible atomic motions. The atomically resolved reaction dynamics were 
determined using a Pearson correlation analysis (eq 71) of possible trajectories connecting the structural end points. From an exhaustive basis, this approach found convergence to a set of modes involving the displacement of just three key modes. All the dynamics, involving over 280 different nuclear degrees of freedom or dimensions (including intermolecular reorganization), could all be fit to three reduced reaction coordinates. Most interesting, these coordinates themselves were highly correlated, implying that the slowest mode was dominating the coupling and renormalizing the potential energy surface. This statement is based on the argument that the slow modes cannot respond fast enough to changes in potential energy surface that might arise from the displacement of higher frequency modes. The effective frequency of the slow mode is too low to be coupled and fully correlated to a higher frequency mode. The dominant mode was assigned to be the motion of the $\mathrm{PF}_{6}^{-}$ counterion, which has a large mass and is only spatially bound by an electrostatic potential. In retrospect, this observation makes sense as the photoinduced charge transfer leads to the formation of a very large local electric field, which would Coulombically couple to the counterion. Given the highly polarizable nature of the EDO-TTF moiety and dipole moment, the change in counterion position renormalizes the potential energy surface for the other modes involved. One of the key modes was indeed the flattening of the EDO-TTF rings for increased wave function overlap of the ring-centered $\pi$ orbitals. This overlap is augmented further by the sliding motion of the rings or center of mass. It was surprising that the counterion motion had such a large effect on the molecular positions. The motion of the counterion is reminiscent of an elephant moving into a crowded room: everyone gets out of the way into new positions (with the floor curving under the weight to further influence positions). In a similar vein, as the $\mathrm{PF}_{6}{ }^{-}$is displaced, the change in potential energy surface leads to atomic motions driven by changes in local field and associated steric effects of the $\mathrm{PF}_{6}{ }^{-}$movement. This system has a very complex potential energy surface with contributions from the highly charged ionic environment and high polarizability contribution from the organic molecules within the lattice. The observation that the counterion plays such an important, even dominant role, in directing the lattice repolarization along the electron transfer reaction coordinate could not have been predicted in advance. The important role of the counterion in this class of "organic salts" in affecting material properties has been speculated as there has been a correlation in the onset of superconductivity in these materials. ${ }^{276}$ We now see the effect directly at the atomic level of detail (Movie S2). This experiment gives a direct observation of the reaction forces from the time-dependent velocity of the reduced effective masses of the involved modes, of which the motion/mass of the counterion is very well-defined. Thus, these observations provide a robust test for theory, particularly with respect to examing the role of the counterion in affecting charge distributions and material properties.

The most dramatic example of a molecular movie is the recent work on the photoinduced charge transfer process in the organo-metallic system $\mathrm{Me}_{4} \mathrm{P}\left[\mathrm{Pt}(\mathrm{dmit})_{2}\right]_{2}\left(\mathrm{Me}_{4} \mathrm{P}=\right.$ tetramethylphosphonium, dmit = 1,3-dithiol-2-thione-4,5-dithiolate) or $\operatorname{Ptd}(\mathrm{mit})_{2}$ for short. ${ }^{37}$ This system undergoes a charge separation phase transition similar to EDO-TTF. The photoinduced charge transfer process occurs through a metal-tometal charge transfer band and thus represents a metal-to-metal electron transfer process, albeit involving the Pt dimer pairs.
This reaction again is in the spirit of Figure 1a, except now the bath is not a liquid but a well-defined lattice to enable a direct observation of the "solvent" coordinate in electron transfer. The system is comparable to ionic solutions but without the broad inhomogeneous distribution of possible intermolecular solvation configurations in a liquid that would blur out the details. As discussed in the introduction, electron transfer represents one of the simplest reactions with no bonds made or broken. The reaction involves only intramolecular and medium repolarization in stabilizing the change in electron distribution. Analogous reactions such as homogeneous electron transfer between iron centers in solution phase (Figure 1A) serve as central concepts for understanding the very large range of homogeneous electron transfer processes that define redox chemistry and electron exchange involved in electrochemistry.

As with EDO-TTF, the highly ionic character of the lattice is prone to changes in charge distribution that drive interconversion between competing stability points in the lattice potential. Previous optical studies have speculated that the optically excited system relaxes into a so-called hidden phase, a metastable state corresponding to the high-temperature phase. The ability to excute femtosecond transient absorption studies of single crystals under the same conditions as the electron diffraction studies was essential to correlating the excited electronic state dynamics to the structural dynamics. The optical excitation into the metal-metal charge transfer band, upon medium repolarization, traps the structure in the metastable state. This process involves a localized transition that should be viewed as an intermolecular charge transfer process. The very important feature of this work is that the unit cell has dimensions up to $3.7 \mathrm{~nm}$, which puts it on par with proteins. This work would not have been possible without the increased electron source brightness. In this case, the electron source was based on a compact electron gun concept to avoid rf timing jitter and complexity yet achieve similar temporal brightness. The gun design was pushed to the physical limit by going to a lens configuration after the sample for the smallest possible propagation distance from the photocathode to the sample while still retaining sufficient transverse coherence for high-quality diffraction from such a large unit cell. ${ }^{49,51,52}$ This work was also the first to find a model-independent solution to invert the diffraction data to real space without the need to use theoretical constraints. Instead, the systems own, known, constraints were used to solve the phase problem (section 2.9, eqs 72-78).

The photoinduced motions are those coupled to the charge transfer coordinate and stabilize the charge-separated state. The atomic movie capturing these events is stunning. Rather than describe specific details, it is best to observe the femtosecondresolved structural changes directly. Movie S3 is derived from experimental diffraction data in which the atomic positions were determined using the new inversion method described above in eqs $72-78$. It is important to emphasize that this atomic movie of a metal-to-metal electron transfer process does not rely on a model for refinement. As discussed above, the differential detection of structural changes is different than trying to solve an unknown structure from diffraction data. The known initial structure and conserved bond angles and rational progression of motions from reactant to product states provided enough constraints to give a unique inversion to real space. There was no need for simple models or theory for a refinement step, which may lead to unintended bias in the reconstruction. The known initial structure and conserved 


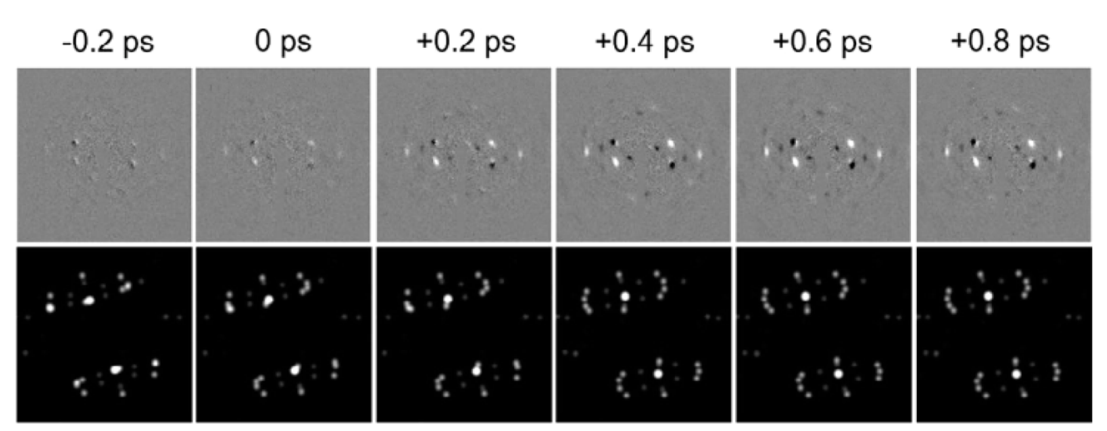

Figure 24. Atom Resolved Dynamics of Photoinduced Electron Transfer for Ptd(mit $)_{2}$. Raw differential diffraction data is shown in the upper panels. Lower panels direct transform to real space using eqs 72-78 in which the relative atomic motions are visible on 100 fs time scales, depicting both the intramolecular and intermolecular reorganization stabilizing the photoinduced metal-to-metal electron transfer. Adapted with permission from ref 37. Copyright 2015 American Association for the Advancement of Science.

nuclear coordinates serve mathematically as phasing centers to provide enough information for a unique inversion to real space. This movie comes directly from the data and as such is the first full atom-resolved chemical reaction. Both the spatial and the temporal resolution were sufficient to capture the primary femtosecond (100 fs) time scale motions governing the dynamics.

The human brain is extremely good at seeing spatial correlations in time as part of rationalizing causal connections. This ability makes movies a particularly powerful means to convey concepts. To not introduce any bias, the atomic positions are presented in the movie as starlike objects on a night sky. The electron transfer involves two asymmetric Pt dimers. One can literally see them oscillate by eye. This motion is equally apparent in the raw data, which illustrates the outstanding signal-to-noise of these experiments. The main point is that the motions could have been any out of a large number of possible motions. But the movie shows that there are only a few dominant motions involved. These motions are most readily observable from the time-correlated movement of atoms as seen in the movie but are also readily observable in the few time frames shown in Figure 24. Since this is an electron transfer process, there will be both intramolecular and intermolecular reorganization (medium repolarization) to stabilize the new electron distribution. Thus, there are effectively 1000's of degrees of freedom in this problem, taking into account minimally a single unit cell for the intermolecular contribution to the reaction coordinate. A more detailed analysis of the motion found that there are principally 6 modes, not 1000 's. Within all the different possible nuclear motions coupled to the electron transfer step, the fact that only a few modes dominate the reorganization can be readily understood by the degree of change in electron density at the given sites. The largest changes occur at the Pt metal centered orbitals. The fact that the $\mathrm{Pt}$ motions are the dominant motions involved in stabilizing the charge separation can clearly be seen by eye.

The different classes of motions can be quantified in terms of the space-time evolution as shown in Figures 25 and 26. The SNR was sufficient to enable resolving changes as small as 0.01 $\AA$, which is a testimony to the brightness and stability of the table top compact electron source used for this study. Here it is equally noteworthy to point out that this spatial resolution is comparable to uncertainty-limited rms zero-point motions for small $Z$ atoms. This resolution limit is sufficient to follow even relatively small changes in reorganization energy and is sufficient to track all of the key motions leading to relaxation along reaction coordinates. For example, the net displacement

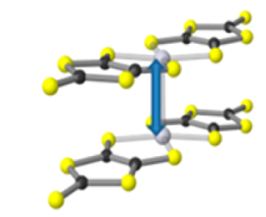

1. Pt...Pt distance

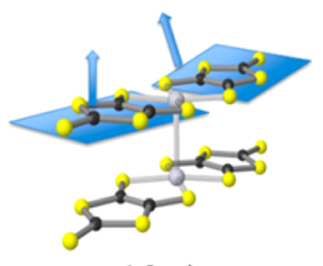

4. Bend

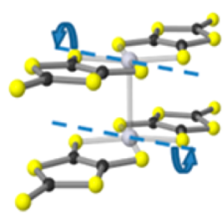

2. Tilt

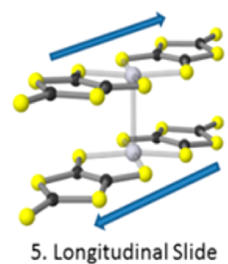

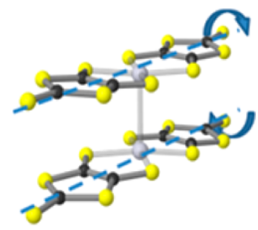

3. Rotation

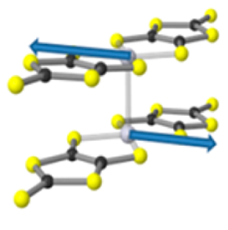

6. Transverse Slide
Figure 25. Six main classifications of motion undergoing reorganization at both intramolecular and intermolecular level subsequent to photoinduced metal-to-metal electron transfer for the organometallic system $\mathrm{Pt}(\mathrm{dmit})_{2}$. Reproduced with permission from ref 37 . Copyright 2015 American Association for the Advancement of Science.

of the central carbon atoms involved in the primary step of vision, as exhibited in the photoisomerization of rhodopsin, is

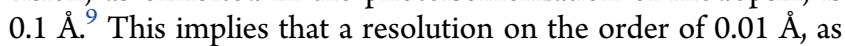
accomplished here, will be needed. In the present case, the resolved motions involve $\mathrm{Pt}$ atoms with large $Z$, which greatly increases the scattering cross section. This scattering center increases the contrast, but the spatial resolution is determined by the crystal quality. Differences in overall diffraction efficiency or scattering cross sections can be compensated for by going to thicker crystals, up to the limit imposed by multiple scattering. The real limit in spatial resolution is the crystal quality and how far, in reciprocal space, the diffraction reaches. This work illustrates that it is possible to resolve motions as small as 0.01 $\AA$ to capture all relevant motions involved in chemical processes. With the advances in diffraction analysis and new means to prepare crystals ${ }^{82}$ giving far more sampling events, it is likely that full atom-resolved dynamics at this same resolution limit can be achieved for all classes of systems. We are now at the fundamental space-time resolution limit needed for studying photoinduced chemical reactions.

The above discussion highlights three molecular reactions where it has been possible to observe an enormous reduction in dimensionality in ever increasingly complex systems to just a few key modes. There are numerous other systems that are nearing completion with respect to their story (classic $\mathrm{I}_{3}{ }^{-}$ reaction, ring opening of spyrooxazine, metal ligand charge 

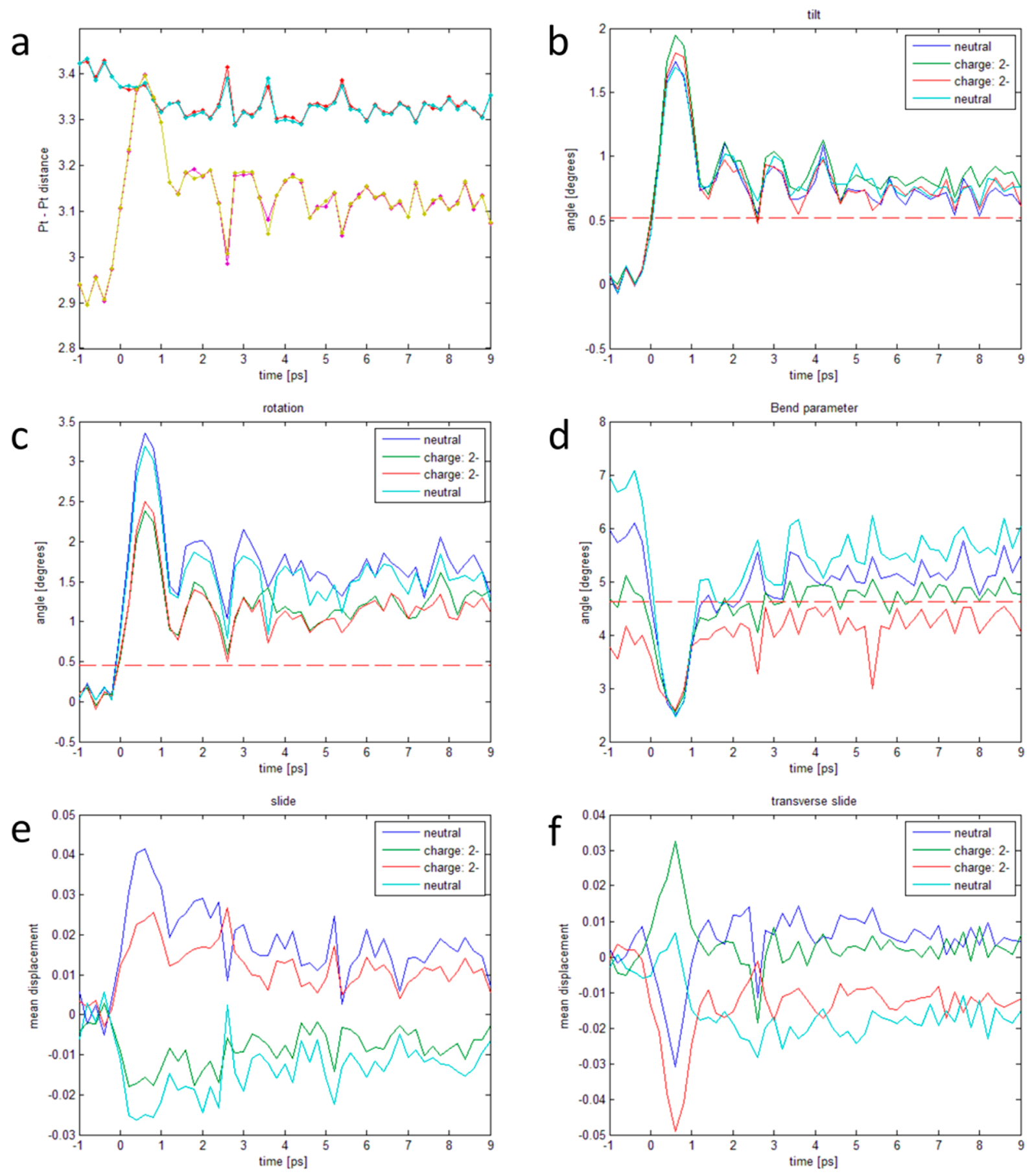

Figure 26. Quantitative analysis of the 6 key classifications of motions involved in reorganization coupled to metal-to-metal electron transfer of the Pt dimers, corresponding to the specific motions shown in Figure 25, a-f correspond chronologically to the different motions 1-6 shown in Figure 25. There are two assymetric Pt dimers, which are colour coded to depict the different motions of the $4 \mathrm{Pt}$ atoms (see ref 37 for details). Adapted with permission from ref 37. Copyright 2015 American Association for the Advancement of Science.

transfer in Fe organometallics, and coupling nuclear motion to electron spin). In all cases, we see the structural transitions involve primarily a few key modes. This is the "magic" of chemistry. During barrier crossing from one stable structure to another, the normal modes become strongly coupled with the lowest frequency, largest amplitude motions, and correspondingly most strongly damped modes directing the transition. The lowest-frequency modes lie within the highest spectral density of states with the highest degree of mixing with bath modes. $^{11,12,103}$ The quantum mechanics of the process is such that the anharmonic coupling between the high-frequency and low-frequency modes within a normal mode basis leads to interference and the creation of localized motions. ${ }^{2}$ It is the anharmonicity of the potential that defines the localization of the fluctuations. The observation of local modes in high overtone IR spectra is a beautiful example of this effect. ${ }^{10,277,278}$ The key point is that this mixing of low-frequency and highfrequency modes leads to the localized motions we call chemistry. 
This enormous reduction in dimensionality to a few key modes emerges from the relatively simple physics depicted in Figure $2 \mathrm{a}$, which makes chemical reaction mechanisms robust. Strictly speaking, these observed reaction mechanisms pertain to the solid state in which the surrounding lattice will impose different barriers to motions along the reaction coordinate say than different lattices or solution phase. The effect of the surrounding lattice will affect the potential energy surface and time scale for the observations, but it is not expected to change the modes involved for a particular molecular system. The effect of the surrounding bath is largest on reactions involving large amplitude motions such as isomerization. Here, for example, the viscosity of the solvent for solution phase processes can retard motion along the torsional coordinate for isomerization. However, the reaction forces still act to displace the same modes, independent of the bath. As a case in point, photoisomerization of retinal in the rhodopsin family of proteins, in which the protein is the bath, involves the same modes as the solution phase and very similar time scales (bond elongation and torsional motion). ${ }^{43}$ The biggest effect will be for cases where the reaction coordinate is primarily determined by the bath as in electron transfer processes (Figure 1a) where intermolecular reorganization (medium repolarization) determines the barrier to charge transfer and dominates the reaction energetics. In this case, the reaction process and specific motions are largely determined by the bath. The dominant modes involved in photoinduced charge transfer process for EDO-TTF and Pt(dmit)2 illustrate this point, with the largest motions involving predominantly intermolecular couplings, distinct to the particular system. Here the bath is the reaction coordinate, yet there are clearly some motions more strongly coupled than others (e.g., $\mathrm{Pt}-\mathrm{Pt}$ distances for $\mathrm{Pt}(\mathrm{dmit}) 2$ and motion of the $\mathrm{PF}_{6}{ }^{-}$counterion for EDO-TTF). Effectively, we observe directly the bath coupling for a well-defined bath configuration. In the case of solution phase charge transfer processes, the same physics will be operating but there will be a distribution of possible nuclear configurations. The specific modes will also differ, depending on the solvent, just as for these two solid state examples. Generally, the most polar highest frequency modes are expected to dominate the dynamics as shown in Figure 1a for librational-type motions. This aspect has been well-established. ${ }^{7}$ It is the specific amplitudes of these overdamped bath modes and effect on the potential energy surface for charge transfer that will be extremely interesting to probe with the next generation of experiments exploiting nanofluidics, as discussed below, to access the solution phase reaction dynamics with electron probes.

Given the very different many-body potentials of different molecules, if it was not for the occurrence of the localization to a few key modes, every molecule would be a new adventure. Chemists have found empirical rules for different reaction mechanisms, lending themselves to "named" reactions. The intuition that experience has built up over time has been based primarily on the rearrangement of closed shell electron configurations (Lewis diagrams) to try to understand changes in charge density. It is understood that the spatial distribution of electron density depends on the specific details of the molecule and that this approach just identifies possible changes in electron distribution. However, this exercise by definition takes a highly localized view of the forces controlling the reaction. There are also mechanisms proposed for the various reactions that form the toolkit of synthetic chemistry, often based on an exhaustive series of synthetically designed probes of reaction outcomes. The proposed mechanisms involve concepts based on changes in electron density and steric factors but ultimately involve interconnected static structures of the reactant and product in which highly localized motions are invoked for the reaction pathway. The fact that this approach works, and has largely shaped the tools used by synthetic chemists, implies that there is something rather profound that has been discovered empirically using primarly synthetic methods to tackle reaction mechanisms. With the relatively recent advances in computational power, this exercise has been complemented by quantum chemistry calculations to better approximate transition state structures. However, as discussed above, the many-body potential involves strong nonadiabatic coupling to nuclear motions and the present theory is not capable of accurately dealing with this problem for systems of chemically interesting scales.

For the first time, we are able to directly observe the farfrom-equilibrium motions that lead to chemical reactions. We can now observe directly this marvelous reduction in dimensionality that has been previously surmised. These experiments will build upon a base that has the potential to change how we think about chemistry. One can foresee the day when we discuss reaction modes associated with certain groups and directly use a dynamic basis to think and construct chemical pathways. In the end, all chemistry is dynamic.

\subsection{Alternative Electron Imaging of Reaction Dynamics:} Rydberg Spectroscopy: Looking from the Inside Out

The discussion to this point has focused on the interaction of free space electrons propagating in nonrelativistic or relativistic regimes to image molecular dynamics in the gas phase or condensed phases. The Coulomb interactions between the traveling electron and the complex electrostatic potential of a molecule cause phase shifts in the wave function of the passing electron. In electron diffraction, these phase shifts are projected onto the distant detector and observed as a diffraction pattern.

An alternative approach in using electron wave functions to observe structural molecular dynamics involves bound electrons that orbit molecular ion cores in Rydberg states. Just like freely traveling electrons, the stationary wave functions of the Rydberg electrons experience phase shifts that arise from Coulomb interactions with all nuclei and electrons of the molecular system. Consequently, the binding energy of a Rydberg electron (i.e., the energy with which the electron is bound to the molecular ion core) is dependent on the molecular structure as shown in Figure $27 .^{279-282}$ The measurement of Rydberg electron binding energies can therefore be used as a form of structural spectroscopy. Because the orbital periods for Rydberg states with modest quantum numbers are much shorter than typical time scales of chemical dynamics, the Rydberg spectroscopy can be implemented in a time-resolved manner.

The electronic spectroscopy of Rydberg states lends itself to the measurement of structural dynamics because the dimensions of Rydberg orbitals exceed those of typical molecules. Consequently, the method can be applied equally to large and small molecules. ${ }^{281}$ Since the number of Rydberg states is governed by basic quantum mechanics of angular momentum states, the complexity of the spectra does not scale with the size of the molecule. Rydberg states are possible for molecules with positive ion cores (i.e., neutral or positively charged molecules). This is because any net positively charged 


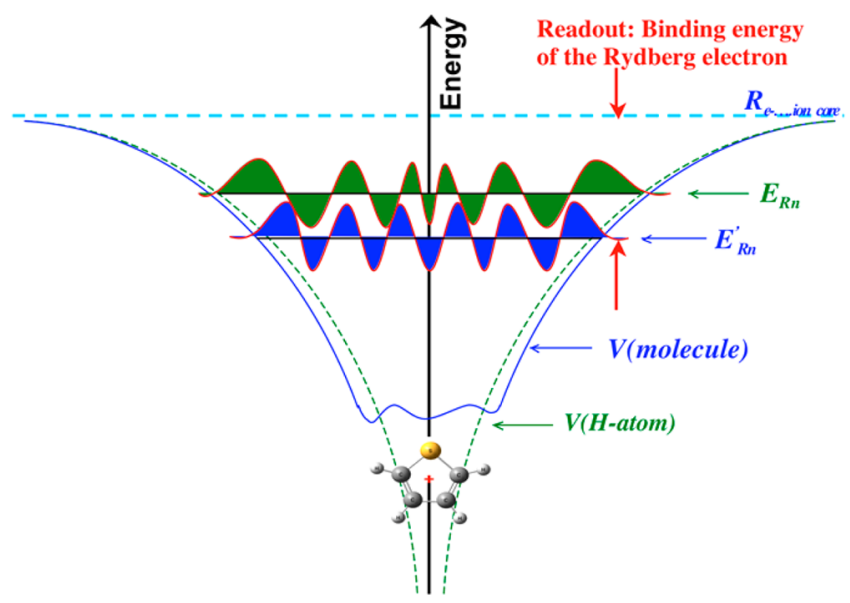

Figure 27. Binding energy of a Rydberg electron (i.e., the energy difference between the vacuum level and the Rydberg orbital) is sensitive to the structure of the molecular ion core because the interactions between the Rydberg electron and the nuclei and electrons of the ion core lead to phase shifts that depend on the position of the elementary particles. Adapted from ref 281. Copyright 2005 American Chemical Society.

molecular ion core exerts a Coulomb attraction to a negatively charged electron that supports Rydberg orbits. Chemical bonding is not necessary to affect the Rydberg electron binding energies: even for weakly bound van der Waals clusters, the effect of a neighboring cluster molecule can be on the order of $0.5 \mathrm{eV},{ }^{283}$ which is easily observed spectroscopically. ${ }^{284}$

While in some molecules, the low $n$ Rydberg states mix with valence states, well-defined Rydberg series start often at $n=3$. The effect of structural changes on the Rydberg electron binding energies at such low quantum numbers are large, so that the spectral resolution attainable with femtosecond lasers is sufficient to record even subtle changes. Isomeric forms of molecular structures have been resolved, ${ }^{281,285,286}$ and even conformeric structures can be separated based on their specific binding energies. ${ }^{287}$ This has made it possible to observe the kinetic reaction sequences ${ }^{288,289}$ and dynamical motions ${ }^{290,291}$ of flexible molecular systems on the complex landscape of conformeric rearrangements.

The Rydberg electron binding energies depend on the distribution of charges in the ion core. Consequently, it has been possible to identify and even select the center of charge in molecules that support two ionization sites. ${ }^{292}$ By following the time evolution of the Rydberg electron binding energy, the reaction of a molecule between different charge states can be observed. ${ }^{293-295}$ Figure 28a illustrates the signatures of dynamic conformeric motions and charge delocalization on the example of $N, N, N, N$-tetramethylethylenediamine. ${ }^{296}$ The molecule can be in a charge-localized structure or in a chargedelocalized structure. The latter has a symmetric geometrical structure with the charge split evenly between the two equivalent nitrogen atoms of the tertiary amine groups. There are several nonequivalent charge-localized structures, which can be identified by comparing the observed binding energies to values computed using self-interaction corrected DFT theory. ${ }^{283}$ Laser excitation prepares the molecule in one of the charge-localized structures, but dynamical motions on the subpicosecond time scale quickly move the system from the Franck-Condon region/geometry to other parts of the potential energy surface belonging to the localized charge
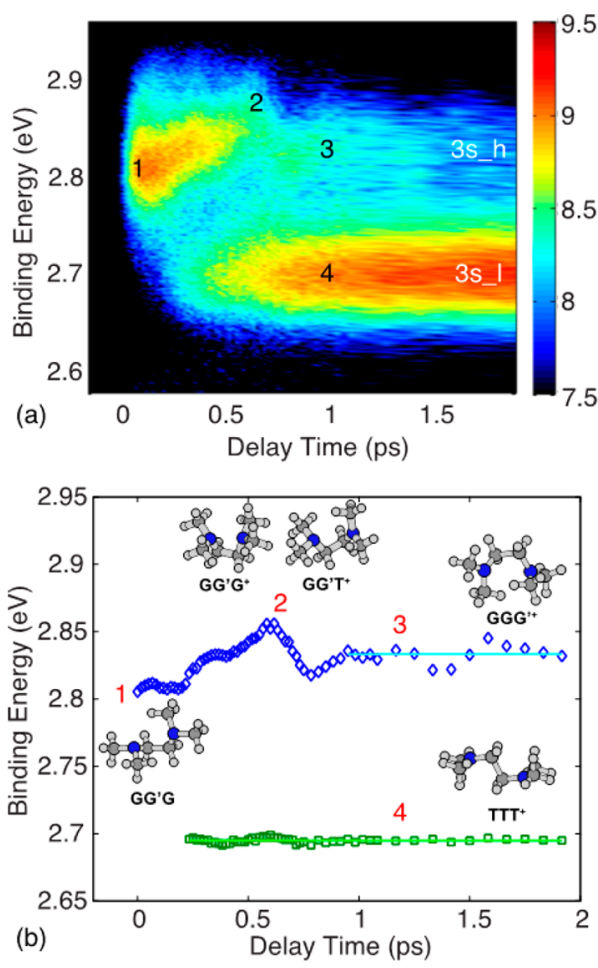

Figure 28. (a) The time-dependent Rydberg electron binding energy spectrum of $N, N, N, N$-tetramethylethylenediamine. Four distinct peak positions are labeled as 1 through 4: three are in the high binding energy region and reflect charge-localized states; one is at lower binding energy and reflects a charge-delocalized state. (b) The centers of the deconvoluted peaks with higher binding energy (blue $\diamond$ and line) and lower binding energy (green $\square$ and line) are identified as localized and delocalized charge states, respectively. The geometrical molecular structures, numbered 1 to 4 , give rise to spectral peak positions as labeled in part (a). Molecular structures are identified by gauche $(\mathrm{G})$ and trans $(\mathrm{T})$ positions for the rotations about single bonds. Reproduced from ref 296. Copyright 2015 American Chemical Society.

states. Some of these structures are indicated in Figure 28b. For the charge to delocalize, a suitable molecular geometry needs to be found. It is the stastical fluctuations of the nuclei and ensuing rearrangement of electrons slaved to this nuclear motion that drives the system to the more stable geometry. As discussed above, it is the sampling of nuclear configurations that determine the operating times scales as is amply demonstrated in these studies. This places the charge delocalization reaction on a picosecond time scale.

The investigation of charge delocalization dynamics is useful because laser excitation prepares molecules in nonequilibrium states. Consequently, the dynamical motions and approach to equilibrium not only reveal structural dynamics of the molecule but also thermodynamic parameters such as the relative energy differences between different charge states. These advances have recently been used to test different computational codes. ${ }^{297}$ It is anticipated that close interaction of experiment with theory will enable the development of advanced functionals that better describe molecular systems with charge delocalization possibilities.

One important reason that the Rydberg spectroscopy can so successfully investigate subtle changes in molecular structure, conformeric dynamics, and charge delocalization is that the spectra are only marginally sensitive to vibrational excitation. 
This is because in most molecules, the potential energy surfaces of the Rydberg states are very similar to those of the ion, so that the entire Franck-Condon envelope is within a narrow spectral bandwidth. As a result, ionization transitions from Rydberg states are measured as well-localized peaks even in systems where valence electronic transitions are broadened by extensive vibrational congestion. ${ }^{298,299}$ Because Rydberg ionization spectra can also be measured at atmospheric pressure, it has been possible to apply the method to investigate transient radicals in flames. A recent study monitored the spatial distribution of methyl radicals in a variety of flames. ${ }^{300}$

Finally, it should be mentioned that there are other means for using internally generated electrons for probing molecular structures on femtosecond time scales. With the advent of attosecond spectroscopy, it is possible to use correlations in high harmonic generation with respect to the generated harmonic profile to image the electronic wave function. ${ }^{44}$ There are multiple interference terms such that this approach relies heavily on theory to make the connection to structure. Similarly, the use of high harmonic generation using phaselocked frequency combs can be used to drive photoelectron emission at energies in the $100 \mathrm{eV}$ range, effectively producing probe electrons internally from the molecule itself with $\AA$ wavelengths. This experimental approach, dubbed laser-induced electron diffraction (LIED) $)^{44,301}$ is similar to Rydberg spectroscopy in its use of internal electrons in the molecular reference frame to probe molecular structure. The difference is the much higher energy of the electrons involved in LIED and much shorter probe wavelength. In this case, the observable is sensitive to vibrations and correlated nuclear motions. The ensuing electron scattering is conceptually similar to electron diffraction with free electron sources as discussed above. The energy range is nearly identical to low energy electron diffraction (LEED). The major difference of LIED from LEED using free-space electrons is that the spatial coherence of the source is not an independent variable that can be fully characterized. There are numerous scattering source terms and $\mathrm{e}-\mathrm{e}$ correlation effects in the photoemission step that makes this analysis reliant on theory to interpret the scattered electron distribution and connect to structural changes. The level of theory needed, and the nonlinear scaling of scattering terms with increasing number of atoms in terms of interpreting the electron scattering, will likely limit this method to small systems. Nevertheless, there are beautiful depictions of correlated atomic motions in response to changes in electron distribution that can now be directly tracked. The major advantages of LIED are that it is completely background free and its extraordinary time resolution. The major disadvantage is that the measurement requires high fields that will strongly perturb the molecular dynamics of interest, which may limit it to the study of photoionization phenomena rather than chemical processes. As a case in point, the most recent example of observing multiphoton ionization and proton dissociation for acetylene (double ionization state) illustrates the dynamics that can be followed. ${ }^{302}$ This experiment involves multiple interference terms to infer proton motion. The same processes involved in chemical reactions, such as keto-enol tautomerization, can be imaged with free space electrons without the strong field perturbation needed to generate the electron source term for the imaging and are directly relevant to the photoinduced chemistry. The unique feature of the attosecond approach is that it opens up the study of high field interactions with the prospect of control of electron dynamics. In this respect, LIED and other attosecond methods provide an additional proving ground for the development of scaleable time-dependent $\mathrm{ab}$ initio theory that will complement related efforts in directly observing atomically resolved reaction dynamics using high brightness free space electrons for imaging chemistry.

\section{SUMMARY AND FUTURE OUTLOOK}

The above discussion provides an overview of the enormous progress that has been made from the very first studies of reactive intermediates using structural probes, to the recognition of the importance of brightness for higher spatial and temporal resolution, to the first atomically resolved reaction dynamics, namely bond formation involved in electrocylization with conserved stereochemistry and intermolecular electron transfer. New analysis procedures have enabled direct inversion of reciprocal space diffraction to real space, giving the first, fully atomically resolved "movie" of a chemical reaction (Figures 24-26). In all cases, we have witnessed the collapse of the nearly innumerable possible nuclear configurations interconnecting structural changes to a few key modes. It is this enormous reduction in dimensionality at reaction saddle points that makes chemistry a transferable concept in terms of manipulating molecular structures. The generalized reaction mechanisms that have been found empirically using synthetic chemistry approaches can now be rationalized in this context. To be clear, the concept of specific reaction modes that propagate systems through barrier crossing regions has long been speculated. The major advance made now is that we can see these motions directly and confirm that this is indeed the mechanism that was missing to explain the general trends in chemistry. Moreover, the specific correlated motions or modes involved could not be determined a priori as the degree of anharmonic coupling in barrier crossing regions can not be projected from the equilibrium structural information on either the reactants or the products. The direct observation of these motions now allows us to categorize chemical reactions by reaction modes, a dynamical basis. The use of particular blocking or leaving groups undoubtedly involves similar fluctuations (reaction modes) that are most strongly coupled to the reaction coordinate in generating seams between reactant and product surfaces. The molecular moiety being manipulated simply provides the bath for the thermal fluctuations driving these modes, creating dynamic pathways between stable minima. It is the extremely strong anharmonicity of the many-body potential in the saddle point region that ultimately couples the particular atomic motions and leads to this reduction in dimensionality, giving rise to generalized reaction mechanisms.

The importance of this advance in conceptualizing chemistry needs to be emphasized in that the systematic displacement of a few key reaction modes provides a highly simplified basis by which one can better reconstruct the nuclear configuration at reaction saddle points (i.e., one should be able to more accurately and in some cases intuitively predict the structure of the transition state region). This ability in turn translates to means to control barrier heights by varying substituents with different charged groups, polarities, and polarizabilities to create potential gradients to both spatially direct these motions and affect the energetics for enhanced control over the desired chemistry. The development of a reaction mode basis to categorize chemistry will provide the needed integration of structure and dynamics to go beyond present depictions of 
reaction mechanisms based on static structures and arrow pushing exercises to rationalize electron redistribution for proposed reaction pathways. The key reaction mode basis will consist of few enough modes that it should lend itself readily to conceptualzing reaction mechanisms well beyond the present methods of planning reaction routes using static structures.

Simply put, the chemists' collective gendanken experiment has been realized. This achievement was accomplished through a number of important technological advances. Foremost among them was the dramatic increase in source brightness for the direct imaging of atomic motions. This breakthrough came from an effectively exact solution to the coupled equations of motions of sufficient electron bunch density for single shot structure determination that laid the foundation for further improvements. There were also important developments in fully characterizing the electron beam spatial and temporal coherence. These advances have enabled the spatial resolution of motions involved in reaction dynamics as small as $0.01 \AA$, which is comparable to zero-point motions. Thus, the goal of spatially resolving chemically relevant atomic motions has been reached. Nevertheless, further improvements are possible. The electron-scattering process enables a direct determination of the nuclear probability distribution for which the centroids could be determined to beyond $10^{-3} \AA$ A. This resolution has already been achieved with long integration measurements in the determination of bond lengths using gas phase electron diffraction. Further increases in source brightness and sample delivery methods will allow the attainment of similar spatial resolution. In this limit, the spatial resolution of the reaction dynamics will then correspond to relative changes in the energetics (i.e., direct determination of the reaction energetics), approaching the resolution of spectroscopic observables.

A further important driver for increased source brightness is the need to study larger systems. How far can the concept of key reaction modes and reduced dimensionality be pushed? How does it scale with complexity and number of degrees of freedom? Chemical strategies exist to organize matter on the nanoscale in order to create active materials. To study such problems relevant to the current state of chemistry implies probing molecular reaction dynamics of larger molecular systems, up to the $\mathrm{kDa}$ scale. Ultimately, one would like to go even beyond this lengths scale, to the level of proteins, to understand how biological systems transduce chemical energy toward specific functions in order to guide the scaling of chemistry to larger organizational principles.

The required increase in source temporal brightness will come from increases in the transverse coherence of electron sources. New photocathode materials give high quantum yields for photoemission with over an order of magnitude reduction in the transverse energy spread over conventional photocathodes $(20 \mathrm{meV}$ relative to $200 \mathrm{meV})$. Another factor of 10 can be gained by going to lower temperatures. ${ }^{59}$ Alternatively, it is possible to use ultracold atoms as electron sources to reach even colder electron distributions. ${ }^{242}$ Overall, there is potentially an increased gain in electron source brightness with respect to spatial resolution by over 2 orders of magnitude. In addition to these developments, new data analysis methods are forthcoming in which more information can be extracted per scattering event. These methods are only now possible with the recent developments in detector technology. With the new direct electron detectors, it is possible to detect single electrons with nearly $100 \%$ quantum efficiency, with SNR ratios of better than 10:1 for single electron detection, and with virtually no detector or read-out noise. This development means that every readout count is real signal and not noise. This development means that very high level correlation methods using intrinsically conserved quantities and causal relationships can be used to extract the maximum information from the scattered electron distributions. It should be possible to attain the same spatial resolution with $10 \times$ fewer electrons and potentially even fewer for image reconstruction of structural changes. In addition, there is the distinct possibility of new technologies to enable going from single shot atomic resolution in a stroboscopic fashion stitching together a movie frame (time point) by frame, to single-shot atom-resolved full movies with $10 \mathrm{fs}$ time resolution. There have been a number of proposals to use streaking methods to project the time variable on an orthogonal direction in the detector plane to collect in "movie mode" to achieve this goal. There are presently geometric limitations in sampling reciprocal space and congestion in the diffraction images. New methods of reconstruction of the atomic structure from the known time projection have solved this problem and will enable the use of longer electron pulses for orders of magnitude more electrons or increased effective imaging brightness. ${ }^{303}$ Taking all these factors into account, the ability to spatially resolve chemical reactions in the next few years will soon be advanced to the true quantum limit for space-time imaging chemistry with an overall improvement in image contrast that would otherwise require more than $10^{3}$ more electrons based on present approaches. Here, quantum limit refers to the uncertainty principle spatial resolution to time-dependent nuclear probability distributions. The future of this field will be bright indeed.

What are the grand challenges in the field of atomically resolved reaction dynamics? One important consideration is the phototrigger step in collecting reaction dynamics. There are very few systems that can be optically prepared on barrierless excited state potentials as required to observe the reaction modes. New methods are needed to drive chemical processes along ground state potential energy surfaces. This could involve rekindling efforts in laser selective chemistry or introducing new means of creating reactive conditions, for example using hot electrons rather than photons to trigger reactions. ${ }^{304}$ Alternatively, methods to create extreme states of matter to explore new regimes of chemistry might open up a new window. The fundamental time resolution and sensitivity limits are interesting questions to ponder. Could we achieve sufficient sensitivity to directly image changes in electron density on the attosecond time scale (the time scale of the electron motion)? This purely electronic contribution to the time-dependent structure factors would be much smaller than the net displacement of electron density with atomic motions. If sufficient sensitivity and temporal resolution could be achieved, we could directly image excited state wavepackets and ponder anew about the absorption of a photon in the creation of superposition states and ensuing mapping onto potential energy surfaces. From the above discussion, the new analysis methods and increasing temporal brightness should make this possible.

The fundamental time resolution limit in this regard is an important question. There are proposals using single electron approaches to reach the attosecond regime while reducing time jitter to a few femtoseconds. ${ }^{254}$ Such pulses have been demonstrated using femtosecond laser pulsed modulation of electron energies followed by compression to create microbunch trains of electron pulses. ${ }^{305}$ There is also evidence for 
attosecond electron pulse trains exploiting the modulation of ultrashort electron pulses with optical nearfields. ${ }^{306}$ These microbunch structured pulse trains are not presently suitable for direct imaging applications but serve as proof of principle with respect to pulse duration. Is it possible to push electron pulse durations below 1 fs with sufficient brightness and accurate timing? In principle, such studies can be conducted in the case of diffraction of isolated relativistic electrons scattered with a characteristic time of $\tau=D_{\text {nucl }} / c=10^{-22}-10^{-23} \mathrm{~s}$. Very similar intrinsic time scales hold for nonrelativistic electrons with energies above $100 \mathrm{keV}$. Only relativistic electrons can be compressed to pulse durations below 1 fs with sufficient spatially extended compressed zones to place a sample and sufficient brightness to become a general observation tool. There are means to use harmonics to reduce the pulse duration below 1 fs with sufficient brightness. ${ }^{61}$ Timing jitter on this scale is a challenge. Nevertheless, one can imagine directly observing the process of creating excited state superposition states with shaped light. For understanding chemistry, the biggest contribution will be to image electron distributions associated with conical intersections. There have been important predictions involving purely quantum effects at conical intersections that such observations would test. At the $\mathrm{CI}$, one would directly observe the breakdown of the BornOppenheimer approximation and the entanglement of electron and nuclear degrees of freedom along the reaction coordinate. This information is important to all chemical reaction dynamics that necessarily involve a transition between different electronic surfaces. These and related studies could lead to major advances in the theory to treat reaction dynamics from first principles. If the source stability and theoretical methods for image reconstruction and refinement can become robust enough to separate nuclear and electronic effects on the structure factors then this possibility could well be realized. This specific point is covered in a recent theoretical treatment of diffraction in the presence of few cycle femtosecond pulses, near field limits to the ionization of graphene as an ideal test system for this concept. ${ }^{307}$

One of the largest problem areas relates to the samples. This reflects in part the above point about phototriggering chemical processes. It would be desirable to expand the sample base to probe different aspects of chemical processes. The targeted synthesis of molecular systems poised to undergo a particular reaction of interest might help. Crystal engineering methods could create the necessarily thin samples on the nanoscale that enable the use of electron probes. This statement also pertains to the study of isolated molecular reaction dynamics in the gas phase where one can foresee the use of molecular cluster methods to build up well-defined bath conditions to probe different aspects of solution phase reactions. The density of such systems is generally low so that increased source brightness will again be important to open up this window of gas phase/molecular cluster reaction dynamics. It will be interesting to observe the redistribution of energy through nonradiative relaxation directly into the surrounding bath molecules, which currently is probed by observing the dynamics of fragmentation of solvent molecules. We could, in principle, observe this directly. The real challenge is to extend the field to in situ studies of homogeneous solution phase chemistry. To this end, new nanofluidic cell technologies have been developed to realize stable liquid pathlengths as thin as $50 \mathrm{~nm}$ within UHV environments. ${ }^{308}$ This development has enabled solution phase studies, but the background scatter from the liquid host medium still masks the small diffraction changes of interest. Here, the new methods for data analysis with differential detection will play an essential role. To date, only X-ray diffraction studies have been conducted in this domain and only for the few systems where sufficiently high solubility keeps the solvent contribution sufficiently small to be factored out of the data. ${ }^{309}$ Electrons can now equally probe solution phase reaction dynamics; however, the problem of dynamic background scattering from the very solvent needed to probe solution phase chemistry will remain a limiting factor.

Finally, heterogeneous chemistry, relevant to applications in catalysis, is an extremely important area for exploration. There has been great progress in developing femtosecond reflected high-energy electron diffraction (RHEED) for surface studies and LEED approaches using nanotips for the shortest propagation distances possible to maintain time resolution. ${ }^{310-313}$ These studies have revealed very interesting cooperative surface effects. In terms of surface chemistry, the main challenge is the irreversible nature of surface reaction dynamics. So far, only reversible processes have been studied in reflection and with low brightness LEED sources to avoid space-charge broadening. For all potential probes of reaction dynamics, the study of surface chemistry using well-defined surfaces under UHV conditions will remain one of the most demanding problems, since even fewer molecular states are probed than in the gas phase and there is no possibility for rapid sample exchange. The problem is even more pronounced as the requirement for exciting at least $10 \%$ of the surface sites to get above background, with the typically small absorption cross sections of surface adsorbates, will lead to the requirement of excitation conditions in excess of $100 \mathrm{GW} /$ $\mathrm{cm}^{2}$ for $100 \mathrm{fs}$ time resolution and associated excitation pulses. This peak power leads to multiphoton ionization, as discussed above, and selective localization of the created charge states in the surface plane. The presence of the charge states changes the surface structure, independent of any presumed excited state dynamics. This makes surfaces very sensitive to charging effects. This issue and effect on diffraction has been discussed in the context of early studies using RHEED geometries to study bulk structural changes. ${ }^{314,315}$ The neglected issue in this discussion is that the generated surface field not only will affect electron scattering (potential for diffraction artifacts) but also will affect the surface structure and potential energy surface (surface state artifacts). The surface selective nature of RHEED and LEED makes these probes particularly prone to any surface charging effects. As an extra consideration, new means of regenerating the reactive surface need to be developed to enable some degree of repetitive sampling the same surface region to enable the use of low excitation to avoid peak powers leading to ionization. Alternatively, model systems using nanoparticles that exploit the same surface chemistry but with large surface areas could provide sufficient number of shots and signal collection to retrieve the structural dynamics.

The great hope for this field is that new means of sample preparation will enable the study of a broad variety of chemical reactions in a systematic fashion, to enable the conceptualization of chemistry based on reaction modes, and to inspire new thinking about how to direct chemical processes. Existing sample constraints will, in all likelihood, only permit the study of a select few reaction mechanisms such as the electrocyclization reactions in relation to bond formation, unimolecular dissociation and isomerization, and intra/intermolecular electron/proton transfer. These are only a small subset of 
the total pool of chemical reaction mechanisms. Most of those cannot be directly probed as the overall mechanism involves multiple steps with long-lived intermediates (which will still be of great interest), and it will not be possible to capture the key reaction modes for each step. Theory will play an everincreasing role in developing a reaction mode basis. With the extremely detailed, atomically resolved reaction dynamics at ever improving space-time resolution, it is expected that concurrent advances in theory will lead to a better understanding of the dynamics. In particular, based on the observed distillation of N-body problems to a few key reaction modes, it will be possible to rigorously determine the minimum construct needed to model the reaction of interest. It should be kept in mind that atomically resolved reaction dynamics is exactly the observable needed to test theoretical predictions. For the most part, theoretical calculations have focused on spectroscopic observables where the accuracy needed in calculating the electronic state energies is quite high. For casting out a reaction mode basis, one only needs to approximate the spatial distribution of forces correctly, as errors in the energetics will likewise affect all competing channels for nuclear rearrangements and energy redistribution. As long as the relative magnitudes of barrier heights and spatial distribution of forces is approximately correct, the correlated atomic motions undergoing the largest displacements and largest coupling to the reaction coordinate should be relatively correct in relation to other competing channels. In this case, it should be possible to go to much larger systems with ab initio methods to develop a generalized reaction mode basis and fully exploit the single, most important, insight coming from atomically resolved reaction dynamics.

The Chemists' Gedanken experiment has been realized, and the basic strategy employed by synthetic chemists has fundamentally been born out. There is an enormous reduction in dimensionality that occurs in the barrier crossing region that leads to strong anharmonic coupling between high and low frequency modes within a normal mode basis. This quantum interference leads to preferential motion along a few reduced reaction coordinates. We can now directly observe these farfrom-equilibrium motions and categorize chemistry in terms of a power spectrum of reaction modes. It is this reduction in dimensionality that makes chemical reaction mechanisms transferrable to seemingly arbitrarily complex (large $N$ ) systems, up to molecules as large as biological macromolecules $(N>1000)$. Chemistry involves key reaction modes, and we now have a new way to reformulate the classic thought experiment using an experimentally derived dynamic mode basis to further refine chemical intuition that has served chemistry so well.

\section{ASSOCIATED CONTENT}

\section{S Supporting Information}

The Supporting Information is available free of charge on the ACS Publications website at DOI: 10.1021/acs.chemrev.6b00770.

Movie S1: atomically resolved electrocyclization processes leading to conserved stereochemistry. Reproduced with permission from ref 2. Copyright 2014 Annual Reviews (AVI)

Movie S2: atomically resolved intermolecular electron transfer leading to charge delocalization in organic systems: EDO-TTF. Reproduced with permission from ref 36. Copyright 2013 Nature Publishing Group (AVI) Movie S3: full atom resolved chemical reaction: intermolecular electron transfer for the $\operatorname{Ptd}(\operatorname{mit}) 2$ organometallic system. Reproduced with permission from ref 37. Copyright 2015 American Association for the Advancement of Science (AVI)

Movie S4: light bullet: laser pondermotive scattering for electron pulse characterization (AVI)

\section{AUTHOR INFORMATION}

\section{Corresponding Author}

*E-mail: dwayne.miller@mpsd.mpg.de.

ORCID 1

Peter M. Weber: 0000-0003-3017-336X

R. J. Dwayne Miller: 0000-0003-0884-0541

Notes

The authors declare no competing financial interest.

\section{ACKNOWLEDGMENTS}

A.A.I. acknowledges support by RFBR Grant 16-29-1167 OFI_m and partial support by Grant 14-22-02035 OFI_m. P.M.W. acknowledges support by the National Science Foundation Grant CBET-1336105, and from DTRA, Grant HDTRA1-14-1-0008. R.J.D.M. would like to thank the Max Planck Society for support and Gastón Corthey, Stuart A. Hayes, Michał A. Kochmann, and Maria S. Grigera for help in preparing this manuscript and providing figures.

\section{REFERENCES}

(1) Polanyi, J. C.; Zewail, A. H. Direct Observation of the Transition State. Acc. Chem. Res. 1995, 28, 119-132.

(2) Miller, R. J. D. Mapping Atomic Motions with Ultrabright Electrons: The Chemists' Gedanken Experiment Enters the Lab Frame. Annu. Rev. Phys. Chem. 2014, 65, 583-604.

(3) Atkins, P.; Paula, J. de. Atkins' Physical Chemistry; OUP Oxford: Oxford, 2010.

(4) Miller, R. J. D. Femtosecond Crystallography with Ultrabright Electrons and X-Rays: Capturing Chemistry in Action. Science 2014, $343,1108-1116$.

(5) Hill, T. L. An Introduction to Statistical Thermodynamics; Courier Dover Publications: New York, 1986.

(6) Conyard, J.; Addison, K.; Heisler, I. A.; Cnossen, A.; Browne, W. R.; Feringa, B. L.; Meech, S. R. Ultrafast Dynamics in the Power Stroke of a Molecular Rotary Motor. Nat. Chem. 2012, 4, 547-551.

(7) Surface Electron Transfer Processes; Miller, R. J. D., Ed.; VCH: New York, 1995.

(8) Kochman, M. A.; Tajti, A.; Morrison, C. A.; Miller, R. J. D. Early Events in the Nonadiabatic Relaxation Dynamics of 4- $(\mathrm{N}, \mathrm{N}-$ Dimethylamino)benzonitrile. J. Chem. Theory Comput. 2015, 11, $1118-1128$

(9) Schapiro, I.; Ryazantsev, M. N.; Frutos, L. M.; Ferré, N.; Lindh, R.; Olivucci, M. The Ultrafast Photoisomerizations of Rhodopsin and Bathorhodopsin Are Modulated by Bond Length Alternation and HOOP Driven Electronic Effects. J. Am. Chem. Soc. 2011, 133, 33543364

(10) Henry, B. R. Use of Local Modes in the Description of Highly Vibrationally Excited Molecules. Acc. Chem. Res. 1977, 10, 207-213.

(11) Dlott, D. D. Optical Phonon Dynamics in Molecular Crystals. Annu. Rev. Phys. Chem. 1986, 37, 157-187.

(12) Pearman, R.; Gruebele, M. On the Importance of Higher Order Anharmonic Molecular Couplings. J. Chem. Phys. 1998, 108, 6561. 
(13) Mahvash, M.; Voo, L. M.; Kim, D.; Jeung, K.; Wainer, J.; Okamura, A. M. Modeling the Forces of Cutting With Scissors. IEEE Trans. Biomed. Eng. 2008, 55, 848-856.

(14) Warshel, A. Bicycle-Pedal Model for the First Step in the Vision Process. Nature 1976, 260, 679-683.

(15) Ramirez, A. P. Colossal Magnetoresistance. J. Phys.: Condens. Matter 1997, 9, 8171.

(16) Hooker, S.; Webb, C. Laser Physics; OUP Oxford: Oxford, 2010.

(17) Quinn, D. M.; Sikorski, R. S. Enzymatic Rate Enhancements. In eLS; John Wiley \& Sons, Ltd, 2001.

(18) Smit, W. A.; Bochkov, A. F.; Caple, R. Organic Synthesis: The Science Behind the Art; Royal Society of Chemistry: Cambridge, 1998.

(19) Eigen, M. Immeasurably Fast Reactions. Nobel Lecture 1967, 11, 170-203.

(20) Neumark, D. M. Transition-State Spectroscopy via Negative Ion Photodetachment. Acc. Chem. Res. 1993, 26, 33-40.

(21) Ischenko, A. A.; Golubkov, V. V.; Spiridonov, V. P.; Zgurskii, A. V.; Akhmanov, A. S.; Vabischevich, M. G.; Bagratashvili, V. N. A Stroboscopical Gas-Electron Diffraction Method for the Investigation of Short-Lived Molecular Species. Appl. Phys. B: Photophys. Laser Chem. 1983, 32, 161-163.

(22) Ischenko, A. A.; Bagratashvili, V. N.; Golubkov, V. V.; Spiridonov, V. P.; Zgurskii, A. V.; Akhmanov, A. S. Moscow Univ. Chem. Bull. 1985, 26, 140 .

(23) Ischenko, A. A.; Spiridonov, V. P.; Tarasov, Y. I.; Stuchebryukhov, A. A. The Cumulant Method in Diffraction Analysis of Polyatomic Molecules. J. Mol. Struct. 1988, 172, 255-273.

(24) Vabischevich, M. G.; Ischenko, A. A. Method of Studying the Kinetics of Fast Processes. Patent of the USSR. 1679907, 1990.

(25) Williamson, J. C.; Dantus, M.; Kim, S. B.; Zewail, A. H. Ultrafast Diffraction and Molecular Structure. Chem. Phys. Lett. 1992, 196, 529534.

(26) Dudek, R. C.; Weber, P. M. Ultrafast Diffraction Imaging of the Electrocyclic Ring-Opening Reaction of 1,3-Cyclohexadiene. J. Phys. Chem. A 2001, 105, 4167-4171.

(27) Williamson, J. C.; Zewail, A. H. Ultrafast Electron Diffraction. Velocity Mismatch and Temporal Resolution in Crossed-Beam Experiments. Chem. Phys. Lett. 1993, 209, 10-16.

(28) Siwick, B. J.; Dwyer, J. R.; Jordan, R. E.; Miller, R. J. D. An Atomic-Level View of Melting Using Femtosecond Electron Diffraction. Science 2003, 302, 1382-1385.

(29) Cao, J.; Hao, Z.; Park, H.; Tao, C.; Kau, D.; Blaszczyk, L. Femtosecond Electron Diffraction for Direct Measurement of Ultrafast Atomic Motions. Appl. Phys. Lett. 2003, 83, 1044.

(30) Rose-Petruck, C.; Jimenez, R.; Guo, T.; Cavalleri, A.; Siders, C. W.; Rksi, F.; Squier, J. A.; Walker, B. C.; Wilson, K. R.; Barty, C. P. J. Picosecond-milliångström Lattice Dynamics Measured by Ultrafast XRay Diffraction. Nature 1999, 398, 310-312.

(31) Williamson, S.; Mourou, G.; Li, J. C. M. Time-Resolved LaserInduced Phase Transformation in Aluminum. Phys. Rev. Lett. 1984, 52, 2364-2367.

(32) Ihee, H.; Lobastov, V. A.; Gomez, U. M.; Goodson, B. M.; Srinivasan, R.; Ruan, C.-Y.; Zewail, A. H. Direct Imaging of Transient Molecular Structures with Ultrafast Diffraction. Science 2001, 291, $458-462$.

(33) Sciaini, G.; Harb, M.; Kruglik, S. G.; Payer, T.; Hebeisen, C. T.; Heringdorf, F.-J. M. zu; Yamaguchi, M.; Hoegen, M. H.; Ernstorfer, R.; Miller, R. J. D. Electronic Acceleration of Atomic Motions and Disordering in Bismuth. Nature 2009, 458, 56-59.

(34) Eichberger, M.; Schäfer, H.; Krumova, M.; Beyer, M.; Demsar, J.; Berger, H.; Moriena, G.; Sciaini, G.; Miller, R. J. D. Snapshots of Cooperative Atomic Motions in the Optical Suppression of Charge Density Waves. Nature 2010, 468, 799-802.

(35) Jean-Ruel, H.; Gao, M.; Kochman, M. A.; Lu, C.; Liu, L. C.; Cooney, R. R.; Morrison, C. A.; Miller, R. J. D. Ring-Closing Reaction in Diarylethene Captured by Femtosecond Electron Crystallography. J. Phys. Chem. B 2013, 117, 15894-15902.

(36) Gao, M.; Lu, C.; Jean-Ruel, H.; Liu, L. C.; Marx, A.; Onda, K.; Koshihara, S.; Nakano, Y.; Shao, X.; Hiramatsu, T.; et al. Mapping
Molecular Motions Leading to Charge Delocalization with Ultrabright Electrons. Nature 2013, 496, 343-346.

(37) Ishikawa, T.; Hayes, S. A.; Keskin, S.; Corthey, G.; Hada, M.; Pichugin, K.; Marx, A.; Hirscht, J.; Shionuma, K.; Onda, K.; et al. Direct Observation of Collective Modes Coupled to Molecular Orbital-driven Charge Transfer. Science 2015, 350, 1501-1505.

(38) Dwyer, J. R.; Hebeisen, C. T.; Ernstorfer, R.; Harb, M.; Deyirmenjian, V. B.; Jordan, R. E.; Miller, R. J. D. Femtosecond Electron Diffraction: "Making the Molecular Movie. Philos. Trans. $R$. Soc., A 2006, 364, 741-778.

(39) Tamura, H.; Nanbu, S.; Ishida, T.; Nakamura, H. Ab Initio Nonadiabatic Quantum Dynamics of Cyclohexadiene/Hexatriene Ultrafast Photoisomerization. J. Chem. Phys. 2006, 124, 084313.

(40) Armstrong, M. R.; Ogilvie, J. P.; Cowan, M. L.; Nagy, A. M.; Miller, R. J. D. Observation of the Cascaded Atomic-to-Global Length Scales Driving Protein Motion. Proc. Natl. Acad. Sci. U. S. A. 2003, 100, 4990-4994.

(41) Maroncelli, M.; Macinnis, J.; Fleming, G. R. Polar Solvent Dynamics and Electron-Transfer Reactions. Science 1989, 243, 16741681.

(42) Miller, R. D. 2000 John C. Polanyi Award Lecture Mother Nature and the Molecular Big Bang. Can. J. Chem. 2002, 80, 1-24.

(43) Johnson, P. J. M.; Halpin, A.; Morizumi, T.; Prokhorenko, V. I.; Ernst, O. P.; Miller, R. J. D. Local Vibrational Coherences Drive the Primary Photochemistry of Vision. Nat. Chem. 2015, 7, 980-986.

(44) Corkum, P. B.; Krausz, F. Attosecond Science. Nat. Phys. 2007, 3, 381-387.

(45) Butler, L. J. Chemical Reaction Dynamics Beyond the BornOppenheimer Approximation. Annu. Rev. Phys. Chem. 1998, 49, 125171.

(46) Spence, J. C. H.; Doak, R. B. Single Molecule Diffraction. Phys. Rev. Lett. 2004, 92, 198102.

(47) Siwick, B. J.; Dwyer, J. R.; Jordan, R. E.; Miller, R. J. D. Ultrafast Electron Optics: Propagation Dynamics of Femtosecond Electron Packets. J. Appl. Phys. 2002, 92, 1643-1648.

(48) Siwick, B. J.; Green, A. A.; Hebeisen, C. T.; Miller, R. J. D. Characterization of Ultrashort Electron Pulses by Electron-Laser Pulse Cross Correlation. Opt. Lett. 2005, 30, 1057.

(49) Hada, M.; Zhang, D.; Pichugin, K.; Hirscht, J.; Kochman, M. A.; Hayes, S. A.; Manz, S.; Gengler, R. Y. N.; Wann, D. A.; Seki, T.; et al. Cold Ablation Driven by Localized Forces in Alkali Halides. Nat. Commun. 2014, 5, 10.1038/ncomms4863.

(50) Hirscht, J. Femtosecond Electron Diffraction: Next Generation Electron Sources for Atomically Resolved Dynamics. Ph.D. Dissertation, Universität Hamburg, 2015.

(51) Gerbig, C.; Senftleben, A.; Morgenstern, S.; Sarpe, C.; Baumert, T. Spatio-Temporal Resolution Studies on a Highly Compact Ultrafast Electron Diffractometer. New J. Phys. 2015, 17, 043050.

(52) Waldecker, L.; Bertoni, R.; Ernstorfer, R. Compact Femtosecond Electron Diffractometer with $100 \mathrm{keV}$ Electron Bunches Approaching the Single-Electron Pulse Duration Limit. J. Appl. Phys. 2015, 117, 044903.

(53) Hebeisen, C. T.; Ernstorfer, R; Harb, M.; Dartigalongue, T.; Jordan, R. E.; Dwayne Miller, R. J. Femtosecond Electron Pulse Characterization Using Laser Ponderomotive Scattering. Opt. Lett. 2006, 31, 3517 .

(54) van der Geer, S. B.; de Loos, M. J.; Vredenbregt, E. J. D.; Luiten, O. J. Ultracold Electron Source for Single-Shot, Ultrafast Electron Diffraction. Microsc. Microanal. 2009, 15, 282-289.

(55) Weber, P. M.; Carpenter, S. D.; Lucza, T. Reflectron Design for Femtosecond Electron Guns. In SPIE's 1995 International Symposium on Optical Science, Engineering, and Instrumentation; International Society for Optics and Photonics, 1995; pp 23-30.

(56) van Oudheusden, T.; de Jong, E. F.; van der Geer, S. B.; Op't Root, W. P. E. M.; Luiten, O. J.; Siwick, B. J. Electron Source Concept for Single-Shot Sub-100 Fs Electron Diffraction in the $100 \mathrm{keV}$ Range. J. Appl. Phys. 2007, 102, 093501. 
(57) Chatelain, R. P.; Morrison, V. R.; Godbout, C.; Siwick, B. J. Ultrafast Electron Diffraction with Radio-Frequency Compressed Electron Pulses. Appl. Phys. Lett. 2012, 101, 081901.

(58) Gao, M.; Jiang, Y.; Kassier, G. H.; Miller, R. J. D. Single Shot Time Stamping of Ultrabright Radio Frequency Compressed Electron Pulses. Appl. Phys. Lett. 2013, 103, 033503.

(59) Cultrera, L.; Karkare, S.; Lee, H.; Liu, X.; Bazarov, I.; Dunham, B. Cold Electron Beams from Cryocooled, Alkali Antimonide Photocathodes. Phys. Rev. Spec. Top.-Accel. Beams 2015, 18, 113401.

(60) Taban, G.; Reijnders, M. P.; Fleskens, B.; van der Geer, S. B.; Luiten, O. J.; Vredenbregt, E. J. D. Ultracold Electron Source for Single-Shot Diffraction Studies. EPL Europhys. Lett. 2010, 91, 46004.

(61) Floettmann, K. Generation of Sub-Fs Electron Beams at FewMeV Energies. Nucl. Instrum. Methods Phys. Res., Sect. A 2014, 740, 34-38.

(62) Hebeisen, C. T.; Sciaini, G.; Harb, M.; Ernstorfer, R.; Dartigalongue, T.; Kruglik, S. G.; Miller, R. J. D. Grating Enhanced Ponderomotive Scattering for Visualization and Full Characterization of Femtosecond Electron Pulses. Opt. Express 2008, 16, 3334.

(63) Morrison, V. R.; Chatelain, R. P.; Godbout, C.; Siwick, B. J. Direct Optical Measurements of the Evolving Spatio-Temporal Charge Density in Ultrashort Electron Pulses. Opt. Express 2013, 21, 21.

(64) Kassier, G. H.; Haupt, K.; Erasmus, N.; Rohwer, E. G.; von Bergmann, H. M.; Schwoerer, H.; Coelho, S. M. M.; Auret, F. D. A Compact Streak Camera for 150 Fs Time Resolved Measurement of Bright Pulses in Ultrafast Electron Diffraction. Rev. Sci. Instrum. 2010, $81,105103$.

(65) Musumeci, P.; Moody, J. T.; Scoby, C. M.; Gutierrez, M. S.; Tran, T. Rf Streak Camera Based Ultrafast Relativistic Electron Diffraction. Rev. Sci. Instrum. 2009, 80, 013302.

(66) Oudheusden, T. van; Nohlmans, J. R.; Roelofs, W. S. C.; Root, W. P. E. M. O.; Luiten, O. J. 3 GHz RF Streak Camera for Diagnosis of Sub-100 Fs, $100 \mathrm{keV}$ Electron Bunches. In Ultrafast Phenomena XVI; Corkum, P.; Silvestri, S.; Nelson, K. A.; Riedle, E.; Schoenlein, R. W., Eds.; Springer Series in Chemical Physics; Springer: Berlin, 2009; pp 938-940.

(67) Hebeisen, C. T.; Sciaini, G.; Harb, M.; Ernstorfer, R.; Kruglik, S. G.; Miller, R. J. D. Direct Visualization of Charge Distributions during Femtosecond Laser Ablation of a $\mathrm{Si}(100)$ Surface. Phys. Rev. B: Condens. Matter Mater. Phys. 2008, 78, 10.1103/PhysRevB.78.081403.

(68) Scoby, C. M.; Li, R. K.; Musumeci, P. Effect of an Ultrafast Laser Induced Plasma on a Relativistic Electron Beam to Determine Temporal Overlap in Pump-probe Experiments. Ultramicroscopy 2013, 127, 14-18.

(69) Manz, S.; Casandruc, A.; Zhang, D.; Zhong, Y.; Loch, R. A.; Marx, A.; Hasegawa, T.; Liu, L. C.; Bayesteh, S.; Delsim-Hashemi, H.; et al. Mapping Atomic Motions with Ultrabright Electrons: Towards Fundamental Limits in Space-Time Resolution. Faraday Discuss. 2015, 177, 467.

(70) Emma, P.; Akre, R.; Arthur, J.; Bionta, R.; Bostedt, C.; Bozek, J.; Brachmann, A.; Bucksbaum, P.; Coffee, R.; Decker, F.-J.; et al. First Lasing and Operation of an Ångstrom-Wavelength Free-Electron Laser. Nat. Photonics 2010, 4, 641-647.

(71) Williams, D. B.; Carter, C. B. Transmission Electron Microscopy: A Textbook for Materials Science, 2nd ed.; Springer: New York, 2009.

(72) Barends, T. R. M.; Foucar, L.; Ardevol, A.; Nass, K.; Aquila, A.; Botha, S.; Doak, R. B.; Falahati, K.; Hartmann, E.; Hilpert, M.; et al. Direct Observation of Ultrafast Collective Motions in CO Myoglobin upon Ligand Dissociation. Science 2015, 350, 445-450.

(73) Pande, K.; Hutchison, C. D. M.; Groenhof, G.; Aquila, A.; Robinson, J. S.; Tenboer, J.; Basu, S.; Boutet, S.; DePonte, D. P.; Liang, M.; et al. Femtosecond Structural Dynamics Drives the Trans/ Cis Isomerization in Photoactive Yellow Protein. Science 2016, 352, 725-729.

(74) Miller, R. J. D. Energetics and Dynamics of Deterministic Protein Motion. Acc. Chem. Res. 1994, 27, 145-150.

(75) Dudek, R. C.; Weber, P. M. Ultrafast Diffraction Imaging of the Electrocyclic Ring-Opening Reaction of 1,3-Cyclohexadiene. J. Phys. Chem. A 2001, 105, 4167-4171.
(76) Dantus, M.; Kim, S. B.; Williamson, J. C.; Zewail, A. H. Ultrafast Electron Diffraction. 5. Experimental Time Resolution and Applications. J. Phys. Chem. 1994, 98, 2782-2796.

(77) Zewail, A. H. Four-Dimensional Electron Microscopy. Science 2010, 328, 187-193.

(78) Baum, P. Towards Ultimate Temporal and Spatial Resolutions with Ultrafast Single-Electron Diffraction. J. Phys. B: At., Mol. Opt. Phys. 2014, 47, 124005.

(79) Aidelsburger, M.; Kirchner, F. O.; Krausz, F.; Baum, P. SingleElectron Pulses for Ultrafast Diffraction. Proc. Natl. Acad. Sci. U. S. A. 2010, 107, 19714-19719.

(80) Bainbridge, A. R.; Barlow Myers, C. W.; Bryan, W. A. Femtosecond Few- to Single-Electron Point-Projection Microscopy for Nanoscale Dynamic Imaging. Struct. Dyn. 2016, 3, 023612.

(81) Zewail, A. H.; Thomas, J. M. 4D Electron Microscopy: Imaging in Space and Time; World Scientific: London, 2010.

(82) Siddiqui, K. M.; Corthey, G.; Hayes, S. A.; Rossos, A.; Badali, D. S.; Xian, R.; Murphy, R. S.; Whitaker, B. J.; Miller, R. J. D. Synchronised Photoreversion of Spirooxazine Ring Opening in Thin Crystals to Uncover Ultrafast Dynamics. CrystEngComm 2016, 18, $7212-7216$.

(83) Suominen, H. J.; Kirrander, A. How to Observe Coherent Electron Dynamics Directly. Phys. Rev. Lett. 2014, 112,10.1103/ PhysRevLett.112.043002.

(84) Kupitz, C.; Basu, S.; Grotjohann, I.; Fromme, R.; Zatsepin, N. A.; Rendek, K. N.; Hunter, M. S.; Shoeman, R. L.; White, T. A.; Wang, D.; et al. Serial Time-Resolved Crystallography of Photosystem II Using a Femtosecond X-Ray Laser. Nature 2014, 513, 261-265.

(85) Sauter, N. K.; Echols, N.; Adams, P. D.; Zwart, P. H.; Kern, J.; Brewster, A. S.; Koroidov, S.; Alonso-Mori, R.; Zouni, A.; Messinger, J.; et al. No Observable Conformational Changes in PSII. Nature 2016, 533, E1-E2.

(86) Snyder, J. W.; Curchod, B. F. E.; Martínez, T. J. GPUAccelerated State-Averaged Complete Active Space Self-Consistent Field Interfaced with $\mathrm{Ab}$ Initio Multiple Spawning Unravels the Photodynamics of Provitamin D3. J. Phys. Chem. Lett. 2016, 7, 24442449.

(87) Sagnella, D. E.; Straub, J. E.; Jackson, T. A.; Lim, M.; Anfinrud, P. A. Vibrational Population Relaxation of Carbon Monoxide in the Heme Pocket of Photolyzed Carbonmonoxy Myoglobin: Comparison of Time-Resolved Mid-IR Absorbance Experiments and Molecular Dynamics Simulations. Proc. Natl. Acad. Sci. U. S. A. 1999, 96, 1432414329.

(88) Bersuker, I. B. Modern Aspects of the Jahn-Teller Effect Theory and Applications To Molecular Problems. Chem. Rev. 2001, 101, 1067-1114.

(89) Bersuker, I. B. Pseudo-Jahn-Teller Effect-A Two-State Paradigm in Formation, Deformation, and Transformation of Molecular Systems and Solids. Chem. Rev. 2013, 113, 1351-1390.

(90) The Jahn-Teller Effect; Köppel, H.; Yarkony, D. R.; Barentzen, H., Eds.; Springer Series in Chemical Physics; Springer: Berlin, 2010; Vol. 97.

(91) Almeida, N. M. S.; McKinlay, R. G.; Paterson, M. J. Computation of Excited States of Transition Metal Complexes. In Computational Studies in Organometallic Chemistry; Macgregor, S. A., Eisenstein, O., Eds.; Structure and Bonding; Springer International Publishing, 2014; pp 107-138.

(92) Worth, G. A.; Cederbaum, L. S. BEYOND BORNOPPENHEIMER: Molecular Dynamics Through a Conical Intersection. Annu. Rev. Phys. Chem. 2004, 55, 127-158.

(93) Pemberton, C. C.; Zhang, Y.; Saita, K.; Kirrander, A.; Weber, P. M. From the (1B) Spectroscopic State to the Photochemical Product of the Ultrafast Ring-Opening of 1,3-Cyclohexadiene: A Spectral Observation of the Complete Reaction Path. J. Phys. Chem. A 2015, $119,8832-8845$.

(94) Ischenko, A. A.; Ogurtsov, I. Y.; Kazantseva, L. A.; Spiridonov, V. P.; Deyanov, R. Z. Gas-Phase Electron Diffraction Investigation of Vibronically Active Molecules: Rhenium Hexafluoride. J. Mol. Struct. 1993, 298, 103-111. 
(95) Ischenko, A. A.; Girichev, G. V.; Tarasov, Y. I. Electron Diffraction: Structure and Dynamics of Free Molecules and Condensed Matter; Fizmatlit: Moscow, 2013.

(96) Ischenko, A. A.; Aseyev, S. A. Time Resolved Electron Diffraction: For Chemistry, Biology And Material Science; Elsevier: San Diego, 2014.

(97) Ryabinkin, I. G.; Joubert-Doriol, L.; Izmaylov, A. F. When Do We Need to Account for the Geometric Phase in Excited State Dynamics? J. Chem. Phys. 2014, 140, 214116.

(98) Mckinlay, R. G.; Żurek, J. M.; Paterson, M. J. Vibronic Coupling in Inorganic Systems: Photochemistry, Conical Intersections, And The Jahn-Teller And Pseudo-Jahn-Teller Effects. In Advances in Inorganic Chemistry; Theoretical and Computational Inorganic Chemistry; Academic Press, 2010; Vol. 62, pp 351-390.

(99) von Neumann, J.; Wigner, E. P. Über das Verhalten von Eigenwerten bei adiabatischen Prozessen. Phys. Z. 1929, 30, 467-470.

(100) Teller, E. Internal Conversion in Polyatomic Molecules. Isr. J. Chem. 1969, 7, 227-235.

(101) Robb, M. A.; Garavelli, M.; Olivucci, M.; Bernardi, F. A Computational Strategy for Organic Photochemistry. In Reviews in Computational Chemistry; Lipkowitz, K. B., Boyd, D. B., Eds.; John Wiley \& Sons, Inc., 2000; pp 87-146.

(102) Paterson, M. J.; Bearpark, M. J.; Robb, M. A.; Blancafort, L.; Worth, G. A. Conical Intersections: A Perspective on the Computation of Spectroscopic Jahn-Teller Parameters and the Degenerate "intersection Space. Phys. Chem. Chem. Phys. 2005, 7, 2100-2115.

(103) Miller, R. J. D. Vibrational Energy Relaxation and Structural Dynamics of Heme Proteins. Annu. Rev. Phys. Chem. 1991, 42, 581614.

(104) Kukura, P.; McCamant, D. W.; Yoon, S.; Wandschneider, D. B.; Mathies, R. A. Structural Observation of the Primary Isomerization in Vision with Femtosecond-Stimulated Raman. Science 2005, 310, 1006-1009.

(105) Feyereisen, M.; Fitzgerald, G.; Komornicki, A. Use of Approximate Integrals in Ab Initio Theory. An Application in MP2 Energy Calculations. Chem. Phys. Lett. 1993, 208, 359-363.

(106) Dunlap, B. I. Robust and Variational Fitting. Phys. Chem. Chem. Phys. 2000, 2, 2113-2116.

(107) Jung, Y.; Sodt, A.; Gill, P. M. W.; Head-Gordon, M. Auxiliary Basis Expansions for Large-Scale Electronic Structure Calculations. Proc. Natl. Acad. Sci. U. S. A. 2005, 102, 6692-6697.

(108) Szalay, P. G.; Watson, T.; Perera, A.; Lotrich, V. F.; Bartlett, R. J. Benchmark Studies on the Building Blocks of DNA. 1. Superiority of Coupled Cluster Methods in Describing the Excited States of Nucleobases in the Franck-Condon Region. J. Phys. Chem. A 2012, $116,6702-6710$.

(109) Szalay, P. G.; Watson, T.; Perera, A.; Lotrich, V.; Fogarasi, G.; Bartlett, R. J. Benchmark Studies on the Building Blocks of DNA. 2. Effect of Biological Environment on the Electronic Excitation Spectrum of Nucleobases. J. Phys. Chem. A 2012, 116, 8851-8860.

(110) Tajti, A.; Szalay, P. G. Analytic Evaluation of the Nonadiabatic Coupling Vector between Excited States Using Equation-of-Motion Coupled-Cluster Theory. J. Chem. Phys. 2009, 131, 124104.

(111) Plasser, F.; Crespo-Otero, R.; Pederzoli, M.; Pittner, J.; Lischka, H.; Barbatti, M. Surface Hopping Dynamics with Correlated Single-Reference Methods: 9H-Adenine as a Case Study. J. Chem. Theory Comput. 2014, 10, 1395-1405.

(112) Tuna, D.; Lefrancois, D.; Wolański, Ł.; Gozem, S.; Schapiro, I.; Andruniów, T.; Dreuw, A.; Olivucci, M. Assessment of Approximate Coupled-Cluster and Algebraic-Diagrammatic-Construction Methods for Ground- and Excited-State Reaction Paths and the ConicalIntersection Seam of a Retinal-Chromophore Model. J. Chem. Theory Comput. 2015, 11, 5758-5781.

(113) Thiel, W. Semiempirical Quantum-chemical Methods. Wiley Interdiscip. Rev. Comput. Mol. Sci. 2014, 4, 145-157.

(114) Weber, W.; Thiel, W. Orthogonalization Corrections for Semiempirical Methods. Theor. Chem. Acc. 2000103, 495-506 $10.1007 / \mathrm{s} 002149900083$.

(115) Koslowski, A.; Beck, M. E.; Thiel, W. Implementation of a General Multireference Configuration Interaction Procedure with
Analytic Gradients in a Semiempirical Context Using the Graphical Unitary Group Approach. J. Comput. Chem. 2003, 24, 714-726.

(116) Warshel, A.; Levitt, M. Theoretical Studies of Enzymic Reactions: Dielectric, Electrostatic and Steric Stabilization of the Carbonium Ion in the Reaction of Lysozyme. J. Mol. Biol. 1976, 103, 227-249.

(117) Makhov, D. V.; Glover, W. J.; Martinez, T. J.; Shalashilin, D. V. $A b$ Initio Multiple Cloning Algorithm for Quantum Nonadiabatic Molecular Dynamics. J. Chem. Phys. 2014, 141, 054110.

(118) Walters, P. L.; Makri, N. Quantum-Classical Path Integral Simulation of Ferrocene-Ferrocenium Charge Transfer in Liquid Hexane. J. Phys. Chem. Lett. 2015, 6, 4959-4965.

(119) Shapiro, M.; Brumer, P.; Shapiro, M. Quantum Control of Molecular Processes, 2nd edition; Wiley-VCH: Weinheim, 2012.

(120) Bergmann, K.; Theuer, H.; Shore, B. W. Coherent Population Transfer among Quantum States of Atoms and Molecules. Rev. Mod. Phys. 1998, 70, 1003-1025.

(121) Warren, W. S.; Rabitz, H.; Dahleh, M. Coherent Control of Quantum Dynamics: The Dream Is Alive. Science 1993, 259, 15811589.

(122) Demiralp, M.; Rabitz, H. Optimally Controlled Quantum Molecular Dynamics: A Perturbation Formulation and the Existence of Multiple Solutions. Phys. Rev. A: At., Mol., Opt. Phys. 1993, 47, 809816.

(123) Kosloff, R.; Rice, S. A.; Gaspard, P.; Tersigni, S.; Tannor, D. J. Wavepacket Dancing: Achieving Chemical Selectivity by Shaping Light Pulses. Chem. Phys. 1989, 139, 201-220.

(124) Bardeen, C. J.; Wang, Q.; Shank, C. V. Selective Excitation of Vibrational Wave Packet Motion Using Chirped Pulses. Phys. Rev. Lett. 1995, 75, 3410-3413.

(125) Bardeen, C. J.; Yakovlev, V. V.; Wilson, K. R.; Carpenter, S. D.; Weber, P. M.; Warren, W. S. Feedback Quantum Control of Molecular Electronic Population Transfer. Chem. Phys. Lett. 1997, 280, 151-158.

(126) Ewbank, J. D.; Schäfer, L.; Ischenko, A. A. Structural and Vibrational Kinetics of Photoexcitation Processes Using Time Resolved Electron Diffraction. J. Mol. Struct. 2000, 524, 1-49.

(127) Ischenko, A. A.; Schafer, L.; Ewbank, J. Time-Resolved Electron Diffraction: A Method to Study the Structural Vibrational Kinetics of Photoexcited Molecules. In Time-resolved Diffraction; Helliwell, J. R., Rentzepis, P. M., Eds.; Oxford University Press: New York, 1997; p 323.

(128) Ischenko, A. A.; Schaefer, L.; Ewbank, J. D. Tomography of the Molecular Quantum State by Time-Resolved Electron Diffraction. In Proc. SPIE 1999; Vol. 3516, pp 580-58710.1117/12.350538. Degtyareva, V. P.; Monastyrski, M. A.; Schelev, M. Y.; Smirnov, A. V., Eds.; 1999.

(129) Cohen-Tannoudji, C.; Diu, B.; Laloe, F. Quantum Mechanics; Wiley: New York, 1987.

(130) Bonham, R. A.; Fink, M. High-Energy Electron Scattering; Van Nostrand Reinhold, 1974.

(131) Hargittai, I. The Gas-Phase Electron Diffraction Technique of Molecular Structure Determination. In Stereochemical Applications of Gas-phase Electron Diffraction;; Hargittai, M., Hargittai, I., Eds.; Wiley$\mathrm{VCH}, 1988$.

(132) Ischenko, A. A.; Schafer, L.; Luo, J. Y.; Ewbank, J. D. Structural and Vibrational Kinetics by Stroboscopic Gas Electron Diffraction: The $193 \mathrm{Nm}$ Photodissociation of CS2. J. Phys. Chem. 1994, 98, $8673-8678$.

(133) Ischenko, A. A.; Ewbank, J. D.; Schafer, L. Structural and Vibrational Kinetics by Time-Resolved Gas Electron Diffraction: Stochastic Approach to Data Analysis. J. Phys. Chem. 1995, 99, 1579015797.

(134) Alvarellos, J.; Metiu, H. The Evolution of the Wave Function in a Curve Crossing Problem Computed by a Fast Fourier Transform Method. J. Chem. Phys. 1988, 88, 4957-4966.

(135) Felker, P. M.; Zewail, A. H. Molecular Structures from Ultrafast Coherence Spectroscopy, in Femtosecond Chemistry. In Femtosecond Chemistry; Manz, J., Wöste, L., Eds.; Wiley-VCH Verlag: Weinheim, 2008. 
(136) Ischenko, A. A.; Schäfer, L.; Ewbank, J. D. Structural Kinetics by Time-Resolved Gas Electron Diffraction: Coherent Nuclear Dynamics in Laser Excited Spatially Anisotropic Molecular Ensembles. J. Mol. Struct. 1996, 376, 157-171.

(137) Centurion, M.; Reckenthaeler, P.; Krausz, F.; Fill, E. Picosecond Electron Diffraction from Molecules Aligned by Dissociation. J. Mol. Struct. 2010, 978, 141-146.

(138) Ogurtsov, I. Y.; kazantseva, L. A.; Ischenko, A. A. Vibronic Effects in Elastic Scattering of Electrons. J. Mol. Struct. 1977, 41, 243251.

(139) Bartell, L. S. Status of Electron Scattering Theory with Respect to Accuracy in Structural Analyses. In Stereochemical Applications of Gas-Phase Electron Diffraction.; Hargittai, I., Hargittai, M., Eds.; VCH Publishers, 1988.

(140) Spiridonov, V. P. Equilibrium Structure and Potential Function: A Goal to Structure Determination. In Advances in Molecular Structure Research; Hargittai, M., Hargittai, I., Eds.; JAI Press Inc: Stamford, 1997; Vol. 3, p 53.

(141) Novosadov, B. K.; Kochikov, I. V.; Tarasov, Y. I. Calculation of Distribution Density Moments for Internuclear Distances in Polyatomic Molecules. Russ. J. Phys. Chem. 2004, 78, 38-42.

(142) Novosadov, B. K.; Kochikov, I. V.; Tarasov, Y. I. Theory and Calculations of Second-Order Anharmonic Corrections to the Radial Distribution Function of Polyatomic Molecules. Russ. J. Phys. Chem. 2006, 80, 1281-1284.

(143) Kochikov, I. V.; Tarasov, Y. I.; Spiridonov, V. P.; Kuramshina, G. M.; Rankin, D. W. H.; Saakjan, A. S.; Yagola, A. G. The Equilibrium Structure of Thiophene by the Combined Use of Electron Diffraction, Vibrational Spectroscopy and Microwave Spectroscopy Guided by Theoretical Calculations. J. Mol. Struct. 2001, 567-568, 29-40.

(144) Born, M.; Oppenheimer, R. Zur Quantentheorie Der Molekeln. Ann. Phys. 1927, 389, 457-484.

(145) Born, M. Kopplung der Elektronen- und Kernbewegung in Molekeln und Kristallen. Nachr. Akad. Wiss. Göttingen, Math.-Phys. Kl $1951,6,1$.

(146) Born, M.; Huang, K. Dynamical Theory of Crystal Lattices; Oxford University Press: Oxford, 1954.

(147) Braun, P. A.; Kiselev, A. A. Introduction to the Theory of Molecular Spectra; Leningrad University: Leningrad, 1983.

(148) Gribov, L. A.; Dementev, V. A. Methods and Algorithms for Making Calculations in the Theory of the Vibrational Spectra of Molecules; Nauka: Moscow, 1981.

(149) Kochikov, I. V.; Tarasov, Y. I. Equilibrium Structure and Internal Rotation in B2F4 from Electron Diffraction and Spectroscopic Data and Quantum Chemical Calculations. Struct. Chem. 2003, 14, 227-238.

(150) Kovtun, D. M.; Kochikov, I. V.; Tarasov, Y. I. Electron Diffraction Analysis for the Molecules with Multiple Large-Amplitude Motions. 3-Nitrostyrene-A Molecule with Two Internal Rotors. J. Phys. Chem. A 2015, 119, 1657-1665.

(151) Kochikov, I. V.; Tarasov, Y. I.; Vogt, N.; Spiridonov, V. P. Large-Amplitude Motion in 1,4-Cyclohexadiene and 1,4-Dioxin: Theoretical Background for Joint Treatment of Spectroscopic, Electron Diffraction and Ab Initio Data. J. Mol. Struct. 2002, 607, $163-174$.

(152) Rapp, D.; Kassal, T. Theory of Vibrational Energy Transfer between Simple Molecules in Nonreactive Collisions. Chem. Rev. 1969, 69, 61-102.

(153) Levine, R. D.; Bernstein, R. B. Molecular Reaction Dynamics; Oxford Univ. Press: New York, 1974.

(154) Herzberg, G. Electronic Spectra of Polyatomic Molecules; Van Nostrand: New York, 1966.

(155) Yang, J.; Guehr, M.; Shen, X.; Li, R.; Vecchione, T.; Coffee, R.; Corbett, J.; Fry, A.; Hartmann, N.; Hast, C.; et al. Diffractive Imaging of Coherent Nuclear Motion in Isolated Molecules. Phys. Rev. Lett. 2016, 117, 153002 .

(156) Tikhonov, A. N.; Arsenin, V. Y. Methods for Solving Ill-Posed Problems; Nauka: Moscow, 1979.
(157) Tikhonov, A. N.; Goncharsky, A. V.; Stepanov, V. V.; Yagola, A. G. Numerical Methods for Solving Ill-Posed Problems; Nauka: Moscow, 1990.

(158) Tikhonov, A. N.; Leonov, A. S.; Yagola, A. G. Non-Linear IllPosed Problems; Nauka: Moscow, 1995.

(159) Miller, K. Stabilized Numerical Analytic Prolongation with Poles. SIAM J. Appl. Math. 1970, 18, 346-363.

(160) Miller, K.; Viano, G. A. On the Necessity of Nearly-BestPossible Methods for Analytic Continuation of Scattering Data. J. Math. Phys. 1973, 14, 1037-1048.

(161) Bertero, M.; Mol, C. D.; Viano, G. A. The Stability of Inverse Problems. In Inverse Scattering Problems in Optics; Baltes, P. D. H. P., Ed.; Topics in Current Physics; Springer: Berlin, 1980; pp 161-214.

(162) Ho, T.; Rabitz, H. Determination of the Interatomic Potential from Elastic Differential Cross Sections at Fixed Energy: Functional Sensitivity Analysis Approach. J. Chem. Phys. 1989, 90, 1519-1525.

(163) Ho, T. S.; Rabitz, H. Inversion of Experimental Data to Extract Intermolecular and Intramolecular Potentials. J. Phys. Chem. 1993, 97, 13447-13456.

(164) Minitti, M. P.; Budarz, J. M.; Kirrander, A.; Robinson, J.; Lane, T. J.; Ratner, D.; Saita, K.; Northey, T.; Stankus, B.; Cofer-Shabica, V.; et al. Toward Structural Femtosecond Chemical Dynamics: Imaging Chemistry in Space and Time. Faraday Discuss. 2014, 171, 81-91.

(165) Lanzafame, J. M.; Miller, R. J. D.; Muenter, A. A.; Parkinson, B. A. Ultrafast Charge-Transfer Dynamics at Tin Disulfide Surfaces. J. Phys. Chem. 1992, 96, 2820-2826.

(166) Coppens, P.; Pitak, M.; Gembicky, M.; Messerschmidt, M.; Scheins, S.; Benedict, J.; Adachi, S.; Sato, T.; Nozawa, S.; Ichiyanagi, K.; et al. The RATIO Method for Time-Resolved Laue Crystallography. J. Synchrotron Radiat. 2009, 16, 226-230.

(167) Zou, X.; Hovmoller, S.; Oleynikov, P. Electron Crystallography: Electron Microscopy and Electron Diffraction; Oxford University Press: Oxford, 2011.

(168) Vorontsov, I. I.; Coppens, P. On the Refinement of TimeResolved Diffraction Data: Comparison of the Random-Distribution and Cluster-Formation Models and Analysis of the Light-Induced Increase in the Atomic Displacement Parameters. J. Synchrotron Radiat. 2005, 12, 488-493.

(169) Gao, M. Mapping Organic Molecular Motions with RF Compressed Femtosecond Electron Diffraction. Ph.D. Thesis, University of Toronto, 2014.

(170) Kirkland, E. J. Advanced Computing in Electron Microscopy; Springer Science \& Business Media, 2010.

(171) Vorontsov, I. I.; Graber, T.; Kovalevsky, A. Y.; Novozhilova, I. V.; Gembicky, M.; Chen, Y.-S.; Coppens, P. Capturing and Analyzing the Excited-State Structure of a $\mathrm{Cu}(\mathrm{I})$ Phenanthroline Complex by Time-Resolved Diffraction and Theoretical Calculations. J. Am. Chem. Soc. 2009, 131, 6566-6573.

(172) Walczak, M.; Grubmüller, H. BASDet: Bayesian Approach(es) for Structure Determination from Single Molecule X-Ray Diffraction Images. Comput. Phys. Commun. 2016, 201, 159-166.

(173) Bagratashvili, V. N.; Doljikov, V. S.; Letokhov, V. S.; Ryabov, E. A. Isotopic Selectivity of IR Laser Photodissociation of CF3I Molecules. Appl. Phys. 1979, 20, 231-235.

(174) Golubkov, V. V.; Zgursky, A. V.; Ischenko, A. A.; Petrov, V. I.; Spiridonov, V. P. Pulse-Resonance Method for Detecting a Signal in a Stroboscopic Electron Microscopy. Abstracts XII All-Union Conference on Electron Microscopy; Nauka: Moscow, 1982; p 62.

(175) Hicks, R. G. What's New in Stable Radical Chemistry? Org. Biomol. Chem. 2006, 5, 1321-1338.

(176) Griller, D.; Ingold, K. U. Persistent Carbon-Centered Radicals. Acc. Chem. Res. 1976, 9, 13-19.

(177) Schafer, L. Electron Diffraction Studies of Free Radicals. I. Indenyl. J. Am. Chem. Soc. 1968, 90, 3919-3925.

(178) Southern, J. F.; Schäfer, L.; Brendhaugen, K.; Seip, H. M. Structural Comparison of Indene and Indenyl Using Electron Diffraction. J. Chem. Phys. 1971, 55, 2418-2421.

(179) Ewbank, J. D.; Faust, W. L.; Luo, J. Y.; English, J. T.; Monts, D. L.; Paul, D. W.; Dou, Q.; Schäfer, L. Instrumentation for Gas Electron 
Diffraction Employing a Pulsed Electron Beam Synchronous with Photoexcitation. Rev. Sci. Instrum. 1992, 63, 3352-3358.

(180) Ewbank, J. D.; Luo, J. Y.; English, J. T.; Liu, R.; Faust, W. L.; Schafer, L. Time-Resolved Gas Electron Diffraction Study of the 193Nm Photolysis of 1,2-Dichloroethenes. J. Phys. Chem. 1993, 97, 87458751.

(181) Golubkov, V. V.; Zgursky, A. V.; Petrov, V. I.; Ischenko, A. A. Pulsed picosecond electron source. Proceedings of the 8th European Congress on Electron Microscopy, Budapest, 1984; pp 61.

(182) Akhmanov, S. A.; Bagratashvili, V. N.; Golubkov, V. V.; Zgurskii, A. V.; Ischenko, A. A.; Krikunov, S. A.; Spiridonov, V. P.; Tunkin, V. G. Generation of the Picosecond Electron Pulses of Fast Electrons in the EMR-100 Electron Diffraction Apparatus by Photoemission in the Laser Field. Russian Journal of Technical Physics Letters 1985, 11, 157-161.

(183) Ihee, H.; Feenstra, J. S.; Cao, J.; Zewail, A. H. Ultrafast Electron Diffraction of Transient Cyclopentadienyl Radical: A Dynamic Pseudorotary Structure. Chem. Phys. Lett. 2002, 353, 325334.

(184) Ihee, H.; Cao, J.; Zewail, A. H. Ultrafast Electron Diffraction: Structures in Dissociation Dynamics of $\mathrm{Fe}(\mathrm{CO}) 5$. Chem. Phys. Lett. 1997, 281, 10-19.

(185) Srinivasan, R.; Lobastov, V. A.; Ruan, C.-Y.; Zewail, A. H. Ultrafast Electron Diffraction (UED). Helv. Chim. Acta 2003, 86, $1761-1799$.

(186) Zewail, A. H. 4d Ultrafast Electron Diffraction, Crystallography, and Microscopy. Annu. Rev. Phys. Chem. 2006, 57, 65-103.

(187) Sadeghi, R. R.; Gwaltney, S. R.; Krause, J. L.; Skodje, R. T.; Weber, P. M. Structure and Dynamics of the S3 State of CS2. J. Chem. Phys. 1997, 107, 6570-6576.

(188) Todd, T. R.; Olson, W. B. The Infrared Spectra of 12C32S, 12C34S, 13C32S, and 12C33S. J. Mol. Spectrosc. 1979, 74, 190-202.

(189) Tzeng, W.-B.; Yin, H.-M.; Leung, W.-Y.; Luo, J.-Y.; Nourbakhsh, S.; Flesch, G. D.; Ng, C. Y. A 193 Nm Laser Photofragmentation Time-of-flight Mass Spectrometric Study of CS2 and CS2 Clusters. J. Chem. Phys. 1988, 88, 1658-1669.

(190) McCrary, V. R.; Lu, R.; Zakheim, D.; Russell, J. A.; Halpern, J. B.; Jackson, W. M. Coaxial Measurement of the Translational Energy Distribution of CS Produced in the Laser Photolysis of CS2 at 193 Nm. J. Chem. Phys. 1985, 83, 3481-3490.

(191) Spence, J. C. H.; Schmidt, K.; Wu, J. S.; Hembree, G.; Weierstall, U.; Doak, B.; Fromme, P. Diffraction and Imaging from a Beam of Laser-Aligned Proteins: Resolution Limits. Acta Crystallogr., Sect. A: Found. Crystallogr. 2005, 61, 237-245.

(192) Chang, Y.-P.; Horke, D. A.; Trippel, S.; Küpper, J. SpatiallyControlled Complex Molecules and Their Applications. Int. Rev. Phys. Chem. 2015, 34, 557-590.

(193) Zon, B. A.; Katsnel'son, B. G. Nonresonant Scattering of Intense Light by a Molecule. Sov. Phys.JETP 1975, 42, 595-601.

(194) Friedrich, B.; Herschbach, D. Alignment and Trapping of Molecules in Intense Laser Fields. Phys. Rev. Lett. 1995, 74, 46234626.

(195) Stapelfeldt, H.; Seideman, T. Colloquium: Aligning Molecules with Strong Laser Pulses. Rev. Mod. Phys. 2003, 75, 543-557.

(196) Ryu, S.; Stratt, R. M.; Weber, P. M. Diffraction Signals of Aligned Molecules in the Gas Phase: Tetrazine in Intense Laser Fields. J. Phys. Chem. A 2003, 107, 6622-6629.

(197) Ryu, S.; Stratt, R. M.; Baeck, K. K.; Weber, P. M. Electron Diffraction of Molecules in Specific Quantum States: A Theoretical Study of Vibronically Excited S-Tetrazine. J. Phys. Chem. A 2004, 108, 1189-1199.

(198) Weber, P. M.; Dudek, R. C.; Ryu, S.; Stratt, R. M. Experimental and Theoretical Studies of Pump-Probe Electron Diffraction: TimeDependent and State-Specific Signatures in Small Cyclic Molecules. In Femtochemistry and Femtobiology; Martin, M., Hynes, J. T., Eds.; Elsevier: Amsterdam, 2004; pp 19-24.

(199) Shorokhov, D.; Park, S. T.; Zewail, A. H. Ultrafast Electron Diffraction: Dynamical Structures on Complex Energy Landscapes. ChemPhysChem 2005, 6, 2228-2250.
(200) Ravindra Kumar, G.; Safvan, C. P.; Rajgara, F. A.; Mathur, D. Intense Laser Field Ionisation of CS2 at $532 \mathrm{Nm}$. Does Dissociation Precede Ionisation? Chem. Phys. Lett. 1994, 217, 626-630.

(201) Banerjee, S.; Kumar, G. R.; Mathur, D. Intensity-Selective, Field-Induced Dissociative Ionization of CS 2 by FemtosecondDuration Light Pulses. J. Phys. B: At., Mol. Opt. Phys. 1999, 32, 4277. (202) Mathur, D.; Bhardwaj, V. R.; Gross, P.; Ravindra Kumar, G.; Rajgara, F. A.; Safvan, C. P.; Vijayalakshmi, K. Angular Distributions of the Products of Dissociative Ionization of Molecules by Intense Laser Fields. Laser Phys. 1997, 7, 829-838.

(203) Iwasaki, A.; Hishikawa, A.; Yamanouchi, K. Real-Time Probing of Alignment and Structural Deformation of CS2 in Intense Nanosecond Laser Fields. Chem. Phys. Lett. 2001, 346, 379-386.

(204) Townsend, D.; Satzger, H.; Ejdrup, T.; Lee, A. M. D.; Stapelfeldt, H.; Stolow, A. B21 $(\Sigma \mathrm{u}+1)$ Excited State Decay Dynamics in CS2. J. Chem. Phys. 2006, 125, 234302.

(205) Kumarappan, V.; Holmegaard, L.; Martiny, C.; Madsen, C. B.; Kjeldsen, T. K.; Viftrup, S. S.; Madsen, L. B.; Stapelfeldt, H. Multiphoton Electron Angular Distributions from Laser-Aligned CS 2 Molecules. Phys. Rev. Lett. 2008, 100, 100.

(206) Bisgaard, C. Z.; Clarkin, O. J.; Wu, G.; Lee, A. M. D.; Geßner, O.; Hayden, C. C.; Stolow, A. Time-Resolved Molecular Frame Dynamics of Fixed-in-Space CS2Molecules. Science 2009, 323, 14641468.

(207) Hockett, P.; Bisgaard, C. Z.; Clarkin, O. J.; Stolow, A. TimeResolved Imaging of Purely Valence-Electron Dynamics during a Chemical Reaction. Nat. Phys. 2011, 7, 612-615.

(208) Fuji, T.; Suzuki, Y.-I.; Horio, T.; Suzuki, T. Excited-State Dynamics of CS2 Studied by Photoelectron Imaging with a Time Resolution of 22 Fs. Chem. - Asian J. 2011, 6, 3028-3034.

(209) Wang, K.; McKoy, V.; Hockett, P.; Schuurman, M. S. TimeResolved Photoelectron Spectra of CS 2: Dynamics at Conical Intersections. Phys. Rev. Lett. 2014, 112, 10.1103/PhysRevLett.112.113007.

(210) Yang, J.; Makhija, V.; Kumarappan, V.; Centurion, M. Reconstruction of Three-Dimensional Molecular Structure from Diffraction of Laser-Aligned Molecules. Struct. Dyn. 2014, 1, 044101.

(211) Yang, J.; Beck, J.; Uiterwaal, C. J.; Centurion, M. Imaging of Alignment and Structural Changes of Carbon Disulfide Molecules Using Ultrafast Electron Diffraction. Nat. Commun. 2015, 6, 8172

(212) Srinivasan, R.; Feenstra, J. S.; Park, S. T.; Xu, S.; Zewail, A. H. Dark Structures in Molecular Radiationless Transitions Determined by Ultrafast Diffraction. Science 2005, 307, 558-563.

(213) Reckenthaeler, P.; Centurion, M.; Fuß, W.; Trushin, S. A.; Krausz, F.; Fill, E. E. Time-Resolved Electron Diffraction from Selectively Aligned Molecules. Phys. Rev. Lett. 2009, 102, 213001.

(214) Hensley, C. J.; Yang, J.; Centurion, M. Imaging of Isolated Molecules with Ultrafast Electron Pulses. Phys. Rev. Lett. 2012, 109, 133202.

(215) Kalal, M.; Nugent, K. Abel Inversion Using Fast Fourier Transforms. Appl. Opt. 1988, 27, 1956.

(216) Dennison, D. M.; Wright, N. A New Long Wave-Length Absorption Band of C S 2. Phys. Rev. 1931, 38, 2077-2078.

(217) Dennison, D. M. The Vibrational Levels of Linear Symmetrical Triatomic Molecules. Phys. Rev. 1932, 41, 304-312.

(218) Deb, S.; Weber, P. M. The Ultrafast Pathway of PhotonInduced Electrocyclic Ring-Opening Reactions: The Case of 1,3Cyclohexadiene. Annu. Rev. Phys. Chem. 2011, 62, 19-39.

(219) Arruda, B. C.; Sension, R. J. Ultrafast Polyene Dynamics: The Ring Opening of 1,3-Cyclohexadiene Derivatives. Phys. Chem. Chem. Phys. 2014, 16, 4439-4455.

(220) Woodward, R. B.; Hoffmann, R. The Conservation of Orbital Symmetry; Verlag Chemie: Weinheim, 1970.

(221) Nenov, A.; Kölle, P.; Robb, M. A.; de Vivie-Riedle, R. Beyond the van Der Lugt/Oosterhoff Model: When the Conical Intersection Seam and the S1 minimum Energy Path Do Not Cross. J. Org. Chem. 2010, 75, 123-129.

(222) Van der Lugt, W. T. A. M.; Oosterhoff, L. J. Symmetry Control and Photoinduced Reactions. J. Am. Chem. Soc. 1969, 91, 6042-6049. 
(223) Garavelli, M.; Page, C. S.; Celani, P.; Olivucci, M.; Schmid, W. E.; Trushin, S. A.; Fuss, W. Reaction Path of a Sub-200 Fs Photochemical Electrocyclic Reaction. J. Phys. Chem. A 2001, 105, 4458-4469.

(224) Dallinga, G.; Toneman, M. L. H. Electron Diffraction by Gases. The Molecular Structure of 1,3-Cyclohexadiene. J. Mol. Struct. 1967, 1, $11-23$.

(225) Trætteberg, M.; Cyvin, S. J.; Olofsson, B.; Nielsen, P. H. The Single and Double Bonds between sp2-Hybridized Carbon Atoms, as Studied by the Gas Electron Diffraction Method. IV. The Molecular Structure of 1,3-Cyclohexadiene. Acta Chem. Scand. 1968, 22, 23052312

(226) Oberhammer, H.; Bauer, S. H. Structures and Conformations of the Cyclohexadienes. J. Am. Chem. Soc. 1969, 91, 10-16.

(227) Ruan, C.-Y.; Lobastov, V. A.; Srinivasan, R.; Goodson, B. M.; Ihee, H.; Zewail, A. H. Ultrafast Diffraction and Structural Dynamics: The Nature of Complex Molecules far from Equilibrium. Proc. Natl. Acad. Sci. U. S. A. 2001, 98, 7117-7122.

(228) Rudakov, F.; Weber, P. M. Ground State Recovery and Molecular Structure upon Ultrafast Transition through Conical Intersections in Cyclic Dienes. Chem. Phys. Lett. 2009, 470, 187-190. (229) Minitti, M. P.; Budarz, J. M.; Kirrander, A.; Robinson, J. S.; Ratner, D.; Lane, T. J.; Zhu, D.; Glownia, J. M.; Kozina, M.; Lemke, H. T.; et al. Imaging Molecular Motion: Femtosecond X-Ray Scattering of an Electrocyclic Chemical Reaction. Phys. Rev. Lett. 2015, 114, 255501. (230) Budarz, J. M.; Minitti, M. P.; Cofer-Shabica, D. V.; Stankus, B.; Kirrander, A.; Hastings, J. B.; Weber, P. M. Observation of Femtosecond Molecular Dynamics via Pump-probe Gas Phase XRay Scattering. J. Phys. B: At., Mol. Opt. Phys. 2016, 49, 034001.

(231) Michalik, A. M.; Sipe, J. E. Analytic Model of Electron Pulse Propagation in Ultrafast Electron Diffraction Experiments. J. Appl. Phys. 2006, 99, 054908.

(232) Geer, S. B. van der; Loos, M. J. de. General Particle Tracer (GPT). http://www.pulsar.nl/gpt/ (accessed Mar 22, 2017).

(233) Floettmann, K. ASTRA, A Space-Charge Tracking Algorithm. http://www.desy.de/ mpyflo/http://www.desy.de/ mpyflo/ (accessed Mar 22, 2017).

(234) Reed, B. W. Femtosecond Electron Pulse Propagation for Ultrafast Electron Diffraction. J. Appl. Phys. 2006, 100, 034916.

(235) Siwick, B. J.; Dwyer, J. R.; Jordan, R. E.; Miller, R. J. D. Femtosecond Electron Diffraction Studies of Strongly Driven Structural Phase Transitions. Chem. Phys. 2004, 299, 285-305.

(236) Kassier, G. H.; Haupt, K.; Erasmus, N.; Rohwer, E. G.; Schwoerer, H. Achromatic Reflectron Compressor Design for Bright Pulses in Femtosecond Electron Diffraction. J. Appl. Phys. 2009, 105, 113111.

(237) Kassier, G. H.; Erasmus, N.; Haupt, K.; Boshoff, I.; Siegmund, R.; Coelho, S. M. M.; Schwoerer, H. Photo-Triggered Pulsed Cavity Compressor for Bright Electron Bunches in Ultrafast Electron Diffraction. Appl. Phys. B: Lasers Opt. 2012, 109, 249-257.

(238) Veisz, L.; Kurkin, G.; Chernov, K.; Tarnetsky, V.; Apolonski, A.; Krausz, F.; Fill, E. Hybrid Dc-ac Electron Gun for Fs-Electron Pulse Generation. New J. Phys. 2007, 9, 451.

(239) Tokita, S.; Hashida, M.; Inoue, S.; Nishoji, T.; Otani, K.; Sakabe, S. Single-Shot Femtosecond Electron Diffraction with LaserAccelerated Electrons: Experimental Demonstration of Electron Pulse Compression. Phys. Rev. Lett. 2010, 105, 215004.

(240) van Oudheusden, T.; Pasmans, P. L. E. M.; van der Geer, S. B.; de Loos, M. J.; van der Wiel, M. J.; Luiten, O. J. Compression of Subrelativistic Space-Charge-Dominated Electron Bunches for SingleShot Femtosecond Electron Diffraction. Phys. Rev. Lett. 2010, 105, 264801.

(241) Gao, M.; Jean-Ruel, H.; Cooney, R. R.; Stampe, J.; de Jong, M.; Harb, M.; Sciaini, G.; Moriena, G.; Dwayne Miller, R. J. Full Characterization of RF Compressed Femtosecond Electron Pulses Using Ponderomotive Scattering. Opt. Express 2012, 20, 12048.

(242) Claessens, B. J.; van der Geer, S. B.; Taban, G.; Vredenbregt, E. J. D.; Luiten, O. J. Ultracold Electron Source. Phys. Rev. Lett. 2005, 95, 164801 .
(243) McCulloch, A. J.; Sheludko, D. V.; Saliba, S. D.; Bell, S. C.; Junker, M.; Nugent, K. A.; Scholten, R. E. Arbitrarily Shaped HighCoherence Electron Bunches from Cold Atoms. Nat. Phys. 2011, 7, 785-788.

(244) Debernardi, N.; van Vliembergen, R. W. L.; Engelen, W. J.; Hermans, K. H. M.; Reijnders, M. P.; van der Geer, S. B.; Mutsaers, P. H. A.; Luiten, O. J.; Vredenbregt, E. J. D. Optimization of the Current Extracted from an Ultracold Ion Source. New J. Phys. 2012, 14, 083011.

(245) Saliba, S. D.; Putkunz, C. T.; Sheludko, D. V.; McCulloch, A. J.; Nugent, K. A.; Scholten, R. E. Spatial Coherence of Electron Bunches Extracted from an Arbitrarily Shaped Cold Atom Electron Source. Opt. Express 2012, 20, 3967-3974.

(246) Engelen, W. J.; van der Heijden, M. A.; Bakker, D. J.; Vredenbregt, E. J. D.; Luiten, O. J. High-Coherence Electron Bunches Produced by Femtosecond Photoionization. Nat. Commun. 2013, 4, 1693.

(247) Sciaini, G.; Miller, R. J. D. Femtosecond Electron Diffraction: Heralding the Era of Atomically Resolved Dynamics. Rep. Prog. Phys. 2011, 74, 096101.

(248) Travier, C. Rf Guns: Bright Injectors for FEL. Nucl. Instrum. Methods Phys. Res., Sect. A 1991, 304, 285-296.

(249) Hastings, J. B.; Rudakov, F. M.; Dowell, D. H.; Schmerge, J. F.; Cardoza, J. D.; Castro, J. M.; Gierman, S. M.; Loos, H.; Weber, P. M. Ultrafast Time-Resolved Electron Diffraction with Megavolt Electron Beams. Appl. Phys. Lett. 2006, 89, 184109.

(250) Musumeci, P.; Moody, J. T.; Scoby, C. M.; Gutierrez, M. S.; Westfall, M. Laser-Induced Melting of a Single Crystal Gold Sample by Time-Resolved Ultrafast Relativistic Electron Diffraction. Appl. Phys. Lett. 2010, 97, 063502.

(251) Li, R.; Huang, W.; Du, Y.; Yan, L.; Du, Q.; Shi, J.; Hua, J.; Chen, H.; Du, T.; Xu, H.; et al. Note: Single-Shot Continuously TimeResolved MeV Ultrafast Electron Diffraction. Rev. Sci. Instrum. 2010, $81,036110$.

(252) Musumeci, P.; Moody, J. T.; Scoby, C. M.; Gutierrez, M. S.; Westfall, M.; Li, R. K. Capturing Ultrafast Structural Evolutions with a Single Pulse of MeV Electrons: Radio Frequency Streak Camera Based Electron Diffraction. J. Appl. Phys. 2010, 108, 114513.

(253) Scoby, C. M.; Musumeci, P.; Moody, J. T.; Gutierrez, M. S. Electro-Optic Sampling at 90 Degree Interaction Geometry for Timeof-Arrival Stamping of Ultrafast Relativistic Electron Diffraction. Phys. Rev. Spec. Top.-Accel. Beams 2010, 13, 022801.

(254) Walbran, M.; Gliserin, A.; Jung, K.; Kim, J.; Baum, P. 5-fs Laser-Electron Synchronization for Pump-Probe Crystallography and Diffraction. Phys. Rev. Appl. 2015, 4, 044013.

(255) Murooka, Y.; Naruse, N.; Sakakihara, S.; Ishimaru, M.; Yang, J.; Tanimura, K. Transmission-Electron Diffraction by $\mathrm{MeV}$ Electron Pulses. Appl. Phys. Lett. 2011, 98, 251903.

(256) Weathersby, S. P.; Brown, G.; Centurion, M.; Chase, T. F.; Coffee, R.; Corbett, J.; Eichner, J. P.; Frisch, J. C.; Fry, A. R.; Gühr, M.; et al. Mega-Electron-Volt Ultrafast Electron Diffraction at SLAC National Accelerator Laboratory. Rev. Sci. Instrum. 2015, 86, 073702.

(257) Siwick, B. J. Femtosecond Electron Diffraction Studies of Strongly-Driven Structural Phase Transitions. Ph.D. thesis, University of Toronto, 2004.

(258) Guo, C.; Rodriguez, G.; Lobad, A.; Taylor, A. J. Structural Phase Transition of Aluminum Induced by Electronic Excitation. Phys. Rev. Lett. 2000, 84, 4493-4496.

(259) Kandyla, M.; Shih, T.; Mazur, E. Femtosecond Dynamics of the Laser-Induced Solid-to-Liquid Phase Transition in Aluminum. Phys. Rev. B: Condens. Matter Mater. Phys. 2007, 75, 214107.

(260) Amini-Nik, S.; Kraemer, D.; Cowan, M. L.; Gunaratne, K.; Nadesan, P.; Alman, B. A.; Miller, R. J. D. Ultrafast Mid-IR Laser Scalpel: Protein Signals of the Fundamental Limits to Minimally Invasive Surgery. PLoS One 2010, 5, e13053.

(261) Petersen, H.; Tavakoli, F.; Kruber, S.; Münscher, A.; Gliese, A.; Hansen, N.-O.; Uschold, S.; Eggert, D.; Robertson, W. D.; Gosau, T.; et al. Comparative Study of Wound Healing in Rat Skin Following Incision with a Novel Picosecond Infrared Laser (PIRL) and Different 
Surgical Modalities: WOUND HEALING AFTER SKIN INCISION VIA PIRL. Lasers Surg. Med. 2016, 48, 385-391.

(262) Böttcher, A.; Clauditz, T. S.; Knecht, R.; Kucher, S.; Wöllmer, W.; Wilczak, W.; Krötz, P.; Jowett, N.; Dalchow, C. V.; Münscher, A.; et al. A Novel Tool in Laryngeal Surgery: Preliminary Results of the Picosecond Infrared Laser: PIRL in Head and Neck Surgery. Laryngoscope 2013, 123, 2770-2775.

(263) Harb, M.; Ernstorfer, R.; Hebeisen, C.; Sciaini, G.; Peng, W.; Dartigalongue, T.; Eriksson, M.; Lagally, M.; Kruglik, S.; Miller, R. Electronically Driven Structure Changes of Si Captured by Femtosecond Electron Diffraction. Phys. Rev. Lett. 2008, 100, 10.1103/ PhysRevLett.100.155504.

(264) Ernstorfer, R.; Harb, M.; Hebeisen, C. T.; Sciaini, G.; Dartigalongue, T.; Miller, R. J. D. The Formation of Warm Dense Matter: Experimental Evidence for Electronic Bond Hardening in Gold. Science 2009, 323, 1033-1037.

(265) Haupt, K.; Eichberger, M.; Erasmus, N.; Rohwer, A.; Demsar, J.; Rossnagel, K.; Schwoerer, H. Ultrafast Metamorphosis of a Complex Charge-Density Wave. Phys. Rev. Lett. 2016, 116, 016402.

(266) Zamponi, F.; Ansari, Z.; Woerner, M.; Elsaesser, T. Femtosecond Powder Diffraction with a Laser-Driven Hard X-Ray Source. Opt. Express 2010, 18, 947.

(267) Woerner, M.; Zamponi, F.; Ansari, Z.; Dreyer, J.; Freyer, B.; Prémont-Schwarz, M.; Elsaesser, T. Concerted Electron and Proton Transfer in Ionic Crystals Mapped by Femtosecond X-Ray Powder Diffraction. J. Chem. Phys. 2010, 133, 064509.

(268) Neubauer, K. R.; Johnston, M. V.; Wexler, A. S. On-Line Analysis of Aqueous Aerosols by Laser Desorption Ionization. Int. J. Mass Spectrom. Ion Processes 1997, 163, 29-37.

(269) Freyer, B.; Zamponi, F.; Juvé, V.; Stingl, J.; Woerner, M.; Elsaesser, T.; Chergui, M. Ultrafast Inter-Ionic Charge Transfer of Transition-Metal Complexes Mapped by Femtosecond X-Ray Powder Diffraction. J. Chem. Phys. 2013, 138, 144504.

(270) Field, R.; Liu, L. C.; Gawelda, W.; Lu, C.; Miller, R. J. D. Spectral Signatures of Ultrafast Spin Crossover in Single Crystal [Fe II $\left.(\text { Bpy })_{3}\right]\left(\mathrm{PF}_{6}\right)_{2}$. Chem. - Eur. J. 2016, 22, 5118-5122.

(271) Irie, M.; Fukaminato, T.; Matsuda, K.; Kobatake, S. Photochromism of Diarylethene Molecules and Crystals: Memories, Switches, and Actuators. Chem. Rev. 2014, 114, 12174-12277.

(272) Jean-Ruel, H.; Cooney, R. R.; Gao, M.; Lu, C.; Kochman, M. A.; Morrison, C. A.; Miller, R. J. D. Femtosecond Dynamics of the Ring Closing Process of Diarylethene: A Case Study of Electrocyclic Reactions in Photochromic Single Crystals. J. Phys. Chem. A 2011, 115, 13158-13168.

(273) Poulin, P. R.; Nelson, K. A. Irreversible Organic Crystalline Chemistry Monitored in Real Time. Science 2006, 313, 1756-1760.

(274) Kochman, M. A.; Bil, A.; Morrison, C. A. Hybrid QM/QM Simulations of Photochemical Reactions in the Molecular Crystal NSalicylidene-2-Chloroaniline. Phys. Chem. Chem. Phys. 2013, 15, 10803-10816.

(275) Chollet, M.; Guerin, L.; Uchida, N.; Fukaya, S.; Shimoda, H.; Ishikawa, T.; Matsuda, K.; Hasegawa, T.; Ota, A.; Yamochi, H.; et al. Gigantic Photoresponse in 1/4-Filled-Band Organic Salt (EDOTTF)2PF6. Science 2005, 307, 86-89.

(276) Jérome, D. The Physics of Organic Superconductors. Science 1991, 252, 1509-1514.

(277) Henry, B. R.; Kjaergaard, H. G. Local Modes. Can. J. Chem. 2002, 80, 1635-1642.

(278) Jensen, P. An Introduction to the Theory of Local Mode Vibrations. Mol. Phys. 2000, 98, 1253-1285.

(279) Kuthirummal, N.; Weber, P. M. Rydberg States: Sensitive Probes of Molecular Structure. Chem. Phys. Lett. 2003, 378, 647-653.

(280) Kuthirummal, N.; Rudakov, F. M.; Evans, C. L.; Weber, P. M. Spectroscopy and Femtosecond Dynamics of the Ring Opening Reaction of 1,3-Cyclohexadiene. J. Chem. Phys. 2006, 125, 133307.

(281) Gosselin, J. L.; Weber, P. M. Rydberg Fingerprint Spectroscopy: A New Spectroscopic Tool with Local and Global Structural Sensitivity. J. Phys. Chem. A 2005, 109, 4899-4904.
(282) Liang, X.; Levy, M. G.; Deb, S.; Geiser, J. D.; Stratt, R. M.; Weber, P. M. Electron Diffraction with Bound Electrons: The Structure Sensitivity of Rydberg Fingerprint Spectroscopy. J. Mol. Struct. 2010, 978, 250-256.

(283) Gudmundsdóttir, H.; Zhang, Y.; Weber, P. M.; Jónsson, H. Self-Interaction Corrected Density Functional Calculations of Rydberg States of Molecular Clusters: N,N-Dimethylisopropylamine. J. Chem. Phys. 2014, 141, 234308.

(284) Deb, S.; Minitti, M. P.; Weber, P. M. Structural Dynamics and Energy Flow in Rydberg-Excited Clusters of N,N-Dimethylisopropylamine. J. Chem. Phys. 2011, 135, 044319.

(285) Cardoza, J. D.; Rudakov, F. M.; Hansen, N.; Weber, P. M. Identification of Isomeric Hydrocarbons by Rydberg Photoelectron Spectroscopy. J. Electron Spectrosc. Relat. Phenom. 2008, 165, 5-10.

(286) Rudakov, F.; Weber, P. M. Ultrafast Structural and Isomerization Dynamics in the Rydberg-Exited Quadricyclane: Norbornadiene System. J. Chem. Phys. 2012, 136, 134303.

(287) Minitti, M. P.; Cardoza, J. D.; Weber, P. M. Rydberg Fingerprint Spectroscopy of Hot Molecules: Structural Dispersion in Flexible Hydrocarbons. J. Phys. Chem. A 2006, 110, 10212-10218.

(288) Minitti, M. P.; Weber, P. M. Time-Resolved Conformational Dynamics in Hydrocarbon Chains. Phys. Rev. Lett. 2007, 98, 10.1103/ PhysRevLett.98.253004.

(289) Deb, S.; Bayes, B. A.; Minitti, M. P.; Weber, P. M. Structural Dynamics in Floppy Systems: Ultrafast Conformeric Motions in Rydberg-Excited Triethylamine. J. Phys. Chem. A 2011, 115, 18041809.

(290) Bush, J. C.; Minitti, M. P.; Weber, P. M. Ultrafast Formation of an Intramolecular Cation-pi Bond. J. Photochem. Photobiol., A 2010, $213,70-72$

(291) Bush, J. C.; Minitti, M. P.; Weber, P. M. Dissociative Energy Flow, Vibrational Energy Redistribution, and Conformeric Structural Dynamics in Bifunctional Amine Model Systems ${ }^{\dagger}$. J. Phys. Chem. A 2010, 114, 11078-11084.

(292) Cheng, W.; Kuthirummal, N.; Gosselin, J. L.; Sølling, T. I.; Weinkauf, R; Weber, P. M. Control of Local Ionization and Charge Transfer in the Bifunctional Molecule 2-Phenylethyl- N, N -Dimethylamine Using Rydberg Fingerprint Spectroscopy. J. Phys. Chem. A 2005, 109, 1920-1925.

(293) Deb, S.; Cheng, X.; Weber, P. M. Structural Dynamics and Charge Transfer in Electronically Excited $N, N^{\prime}$-Dimethylpiperazine. J. Phys. Chem. Lett. 2013, 4, 2780-2784.

(294) Cheng, X.; Zhang, Y.; Deb, S.; Minitti, M. P.; Gao, Y.; Jónsson, H.; Weber, P. M. Ultrafast Structural Dynamics in Rydberg Excited $\mathrm{N}, \mathrm{N}, \mathrm{N}^{\prime}, \mathrm{N}^{\prime}$-Tetramethylethylenediamine: Conformation Dependent Electron Lone Pair Interaction and Charge Delocalization. Chem. Sci. 2014, 5, 4394-4403.

(295) Cheng, X.; Gao, Y.; Rudakov, F.; Weber, P. M. Charge Transfer and Ultrafast Nuclear Motions: The Complex Structural Dynamics of an Electronically Excited Triamine. Chem. Sci. 2016, 7, 619-627.

(296) Cheng, X.; Zhang, Y.; Gao, Y.; Jónsson, H.; Weber, P. M. Ultrafast Structural Pathway of Charge Transfer in $N, N, N^{\prime}, N$ '-Tetramethylethylenediamine. J. Phys. Chem. A 2015, 119, 28132818

(297) Cheng, X.; Zhang, Y.; Jónsson, E.; Jónsson, H.; Weber, P. M. Charge Localization in a Diamine Cation Provides a Test of Energy Functionals and Self-Interaction Correction. Nat. Commun. 2016, 7, 11013.

(298) Cardoza, J. D.; Weber, P. M. Resolved: Electronic States underneath Broad Absorptions. J. Chem. Phys. 2007, 127, 036101.

(299) Cardoza, J. D.; Rudakov, F. M.; Weber, P. M. Electronic Spectroscopy and Ultrafast Energy Relaxation Pathways in the Lowest Rydberg States of Trimethylamine. J. Phys. Chem. A 2008, 112, 10736-10743.

(300) Rudakov, F.; Gao, Y.; Cheng, X.; Weber, P. M. Non-Intrusive Detection of Combustion Intermediates by Photoionization via Rydberg States and Microwave Backscattering. Combust. Flame 2016, $171,162-167$. 
(301) Zuo, T.; Bandrauk, A. D.; Corkum, P. B. Laser-Induced Electron Diffraction: A New Tool for Probing Ultrafast Molecular Dynamics. Chem. Phys. Lett. 1996, 259, 313-320.

(302) Wolter, B.; Pullen, M. G.; Le, A.-T.; Baudisch, M.; DoblhoffDier, K.; Senftleben, A.; Hemmer, M.; Schröter, C. D.; Ullrich, J.; Pfeifer, T.; et al. Ultrafast Electron Diffraction Imaging of Bond Breaking in Di-Ionized Acetylene. Science 2016, 354, 308-312.

(303) Badali, Daniel. Structural Dynamics and Atomic Motion in Thin Films Studied by Ultrafast Electron Diffraction and Transient Optical Spectroscopy. Ph.D. thesis, University of Hamburg, 2015.

(304) Hada, M.; Zhang, D.; Casandruc, A.; Miller, R. J. D.; Hontani, Y.; Matsuo, J.; Marvel, R. E.; Haglund, R. F. Hot Electron Injection Driven Phase Transitions. Phys. Rev. B 2012, 86,13410110.1103/ PhysRevB.86.134101.

(305) Sears, C. M. S.; Colby, E.; Ischebeck, R.; McGuinness, C.; Nelson, J.; Noble, R.; Siemann, R. H.; Spencer, J.; Walz, D.; Plettner, T.; et al. Production and Characterization of Attosecond Electron Bunch Trains. Phys. Rev. Spec. Top.--Accel. Beams 2008, 11, 06130110.1103/PhysRevSTAB.11.061301.

(306) Feist, A.; Echternkamp, K. E.; Schauss, J.; Yalunin, S. V.; Schäfer, S.; Ropers, C. Quantum Coherent Optical Phase Modulation in an Ultrafast Transmission Electron Microscope. Nature 2015, 521, 200-203.

(307) Yakovlev, V. S.; Stockman, M. I.; Krausz, F.; Baum, P. AtomicScale Diffractive Imaging of Sub-Cycle Electron Dynamics in Condensed Matter. Sci. Rep. 2015, 5, 14581.

(308) Mueller, C.; Harb, M.; Dwyer, J. R.; Miller, R. J. D. Nanofluidic Cells with Controlled Pathlength and Liquid Flow for Rapid, HighResolution In Situ Imaging with Electrons. J. Phys. Chem. Lett. 2013, 4, 2339-2347.

(309) Ihee, H. Visualizing Solution-Phase Reaction Dynamics with Time-Resolved X-Ray Liquidography. Acc. Chem. Res. 2009, 42, 356366.

(310) Paarmann, A.; Gulde, M.; Müller, M.; Schäfer, S.; Schweda, S.; Maiti, M.; Xu, C.; Hohage, T.; Schenk, F.; Ropers, C.; et al. Coherent Femtosecond Low-Energy Single-Electron Pulses for Time-Resolved Diffraction and Imaging: A Numerical Study. J. Appl. Phys. 2012, 112, 113109-113110.

(311) Gulde, M.; Schweda, S.; Storeck, G.; Maiti, M.; Yu, H. K.; Wodtke, A. M.; Schafer, S.; Ropers, C. Ultrafast Low-Energy Electron Diffraction in Transmission Resolves Polymer/Graphene Superstructure Dynamics. Science 2014, 345, 200-204.

(312) Frigge, T.; Hafke, B.; Tinnemann, V.; Witte, T.; Horn-von Hoegen, M. Spot Profile Analysis and Lifetime Mapping in Ultrafast Electron Diffraction: Lattice Excitation of Self-Organized Ge Nanostructures on Si(001). Struct. Dyn. 2015, 2, 035101.

(313) Lüneburg, S.; Müller, M.; Paarmann, A.; Ernstorfer, R. Microelectrode for Energy and Current Control of Nanotip Field Electron Emitters. Appl. Phys. Lett. 2013, 103, 213506.

(314) Park, H.; Zuo, J.-M. Comment on "Structural Preablation Dynamics of Graphite Observed by Ultrafast Electron Crystallography. Phys. Rev. Lett. 2010, 105, 059603.

(315) Carbone, F.; Baum, P.; Rudolf, P.; Zewail, A. H.; et al. Carbone et al. Reply: Phys. Rev. Lett. 2010, 105, 105. 